\begin{tabular}{|c|c|c|c|c|c|c|c|c|c|c|}
\hline \multicolumn{4}{|c|}{$\begin{array}{c}\text { 2. To: (Receiving Organization) } \\
\text { Distribution }\end{array}$} & \multicolumn{3}{|c|}{$\begin{array}{l}\text { 3. From: (originating organization) } \\
222-\mathrm{S} \text { Laboratory Complex }\end{array}$} & \multicolumn{4}{|c|}{$\begin{array}{r}\text { 4. Related EDT No.: } \\
\text { N/A }\end{array}$} \\
\hline \multicolumn{4}{|c|}{$\begin{array}{l}\text { 5. Proj./Prog./Dept./Div.: } \\
\text { 222-S Laboratory/Waste } \\
\text { Management/PPC/AS }\end{array}$} & \multicolumn{3}{|c|}{$\begin{array}{l}\text { 6. Design Authority/ Design Agent/Cog. } \\
\text { Engr.: } \\
\text { Cary M. Seidel }\end{array}$} & \multicolumn{4}{|c|}{$\begin{array}{l}\text { 7. Purchase order No.: } \\
\qquad N / A\end{array}$} \\
\hline \multirow{2}{*}{\multicolumn{7}{|c|}{$\begin{array}{l}\text { 8. Originator Remarks: } \\
\text { This document is being released into the supporting document } \\
\text { system for retrievability purposes. }\end{array}$}} & \multicolumn{4}{|c|}{$\begin{array}{l}\text { 9. Equip./Component No.: } \\
\text { N/A }\end{array}$} \\
\hline & & & & & & & \multicolumn{4}{|c|}{$\begin{array}{l}\text { 10. System/Bldg./Facility: } \\
222-\mathrm{S} \text { Laboratory }\end{array}$} \\
\hline \multirow{3}{*}{\multicolumn{4}{|c|}{$\begin{array}{l}\text { 11. Receiver Renarks: } \\
\text { For release. }\end{array}$}} & \multirow{3}{*}{\multicolumn{3}{|c|}{ taseline Document? [] Yes $[X]$ No }} & \multicolumn{4}{|c|}{$\begin{array}{l}\text { 12. Major Assm. Dwg. No.: } \\
\text { N/A }\end{array}$} \\
\hline & & & & & & & \multicolumn{4}{|c|}{$\begin{array}{l}\text { 13. Permit/Permit Application No.: } \\
\qquad / \mathrm{A}\end{array}$} \\
\hline & & & & & & & \multicolumn{4}{|c|}{$\begin{array}{l}\text { 14. Required Response Date: } \\
04 / 22 / 98\end{array}$} \\
\hline \multicolumn{7}{|c|}{ DATA TRANSMITTED } & \multirow[b]{2}{*}{$\begin{array}{l}\text { Approval } \\
\text { Desig. } \\
\text { nator }\end{array}$} & \multirow[b]{2}{*}{$\begin{array}{l}\text { (G) } \\
\text { Reason } \\
\text { for } \\
\text { Trans- } \\
\text { mittal }\end{array}$} & $(H)$ & (1) \\
\hline $\begin{array}{l}\text { (A) } \\
\text { Item } \\
\text { No. }\end{array}$ & \multicolumn{2}{|c|}{ (B) Document/Drawing No. } & $\begin{array}{l}\text { DATA } \\
\text { (C) } \\
\text { Sheet } \\
\text { No. }\end{array}$ & $\begin{array}{l}\text { (D) } \\
\text { Rev. } \\
\text { No. }\end{array}$ & \multicolumn{2}{|c|}{$\begin{array}{l}\text { (E) Title or Description of Data } \\
\text { Iransmitted }\end{array}$} & & & $\begin{array}{l}\text { Origi- } \\
\text { nator } \\
\text { Dispo- } \\
\text { sition }\end{array}$ & $\begin{array}{l}\text { Receiv- } \\
\text { er } \\
\text { Dispo- } \\
\text { sition. }\end{array}$ \\
\hline 1 & HNF-1641 & . & $\mathrm{N} / \mathrm{A}$ & 0 & $\begin{array}{l}\text { Developr } \\
\text { Inventor } \\
\text { Program } \\
\text { Retentic } \\
\text { Managem } \\
\text { Disposi } \\
\text { Charact } \\
\text { Samples } \\
\text { Laborat }\end{array}$ & $\begin{array}{l}\text { of an } \\
\text { rchive } \\
\text { the } \\
\text { and } \\
\text { of Tank } \\
\text { ation } \\
\text { the 222-s }\end{array}$ & $N / A$ & $?$ & 1 & 1 \\
\hline \multicolumn{11}{|c|}{ (1) } \\
\hline \multirow{2}{*}{\multicolumn{2}{|c|}{$\begin{array}{l}\text { Approval Designator }(F) \\
\text { E, S, Q, D or N/A } \\
\text { (see WHC-CM-3-5, } \\
\text { Sec.12.7) }\end{array}$}} & \multicolumn{4}{|c|}{ Reason for Transmittal (G) } & \multicolumn{5}{|c|}{ Disposition (H) \& (I) } \\
\hline & & $\begin{array}{l}\text { 1. Appro } \\
\text { 2. Releas } \\
\text { 3. Inform }\end{array}$ & \multicolumn{2}{|c|}{$\begin{array}{l}\text { 4. Reviow } \\
\text { 5. Post-Review } \\
\text { 6. Dist. (Receipt AC }\end{array}$} & now. Required! & $\begin{array}{l}\text { 1. Approved } \\
\text { 2. Approved w/c } \\
\text { 3. Disapproved }\end{array}$ & $\begin{array}{l}\text { mment } \\
\text { comment }\end{array}$ & \multicolumn{3}{|c|}{$\begin{array}{l}\text { 4. Reviewed no/comment } \\
\text { 5. Reviewed w/comment } \\
\text { 6. Receipt acknowledged }\end{array}$} \\
\hline
\end{tabular}

17. SIGNATURE/DISTRIBUTION

(See Approval Designator for required signatures]

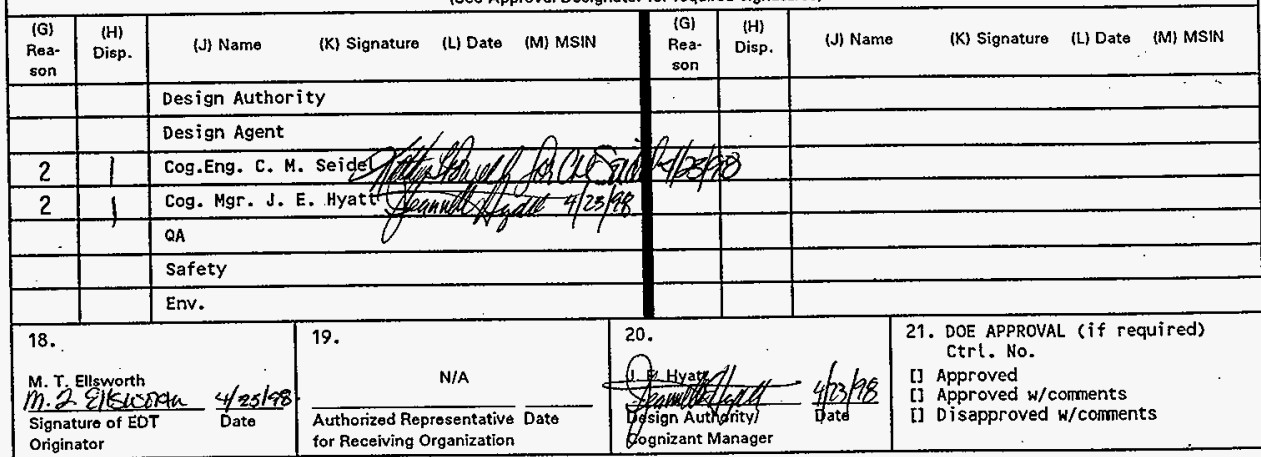




\section{Development of an Inventory/Archive Program for the Retention, Management, and Disposition of Tank Characterization Samples at the 222-S Laboratory}

Cary M. Seidel

Waste Management of Hanford, Inc., Richland, WA 99352

U.S. Department of Energy Contract DE-AC06-96RL13200

Cl ifford T. Narquis

Badger Mountain Technical Associates, West Richland, WA 99353

EDT/ECN: EDT-623151

Org Code: 31120

B\&R Code: EW 3120074
UC: 2070

Charge Code: N4KIE

Total Pages: 179

Key Words: Development, Inventory/Archive, Inventory, Archive, Program, Retention, Management, Disposition, Tank Characterization, Samples, 222-S Laboratory

Abstract: N/A

TRADEMARK DISCLAIMER. Reference herein to any specific commercial product, process, or service by trade name, trademark, manufacturer, or otherwise, does not necessarily constitute or imply its endorsement, recommendation, or favoring by the United States Government or any agency thereof or its contractors or subcontractors.

Printed in the United States of America. To obtain copies of this document, contact: Document Control Services, P.O. Box 950, Mailstop H6-08, Richland WA 99352, Phone (509) 372-2420;

Fax (509) 376-4989.
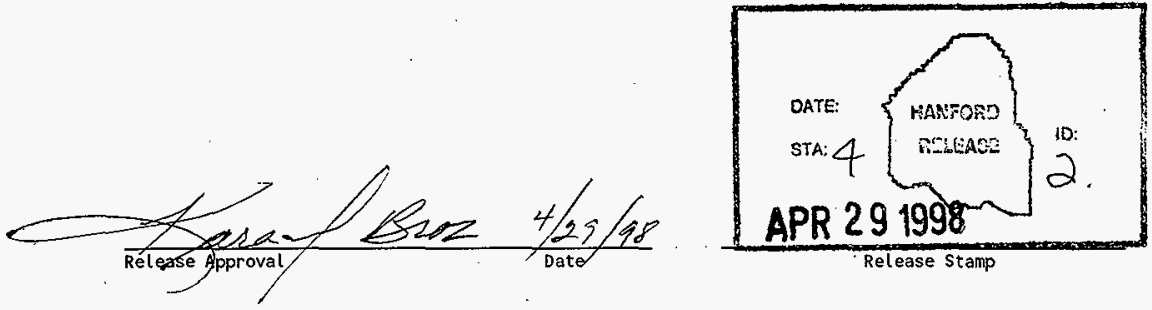


\section{FINAL REPORT}

\section{Development of an Inventory/Archive Program for the Retention, Management, and Disposition of Tank Characterization Samples at the 222-S Laboratory}

Presented to:

Daniel S. Mantooth and Cary M. Seidel

Waste Management Federal Services of Hanford, Inc.

Prepared by:

Clifford T. Narquis

Badger Mountain Technical Associates

April $20 ; 1998$ 
HNF-1641, Rev, 0

TABLE OF CONTENTS

Executive Summary .................... I

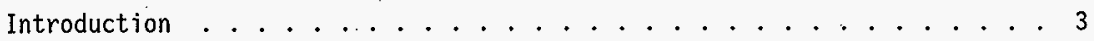

Proposed Archivat Program . . . . . . . . . . . . . 5

Physical Inventory ...................... 10

Containers ........................ 16

Storage Needs and Current Readiness . . . . . . . . . . . . 18

Maintenance of Archived Samples................... 30

Consolidation of Existing Database .................. 34

Disposition .......................... 35

Regulatory Considerations . . . . . . . . . . . 37

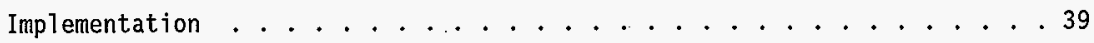

References .................. . . 40

Appendix I (222-S Archived Database Sorted by Tank:Core:Matrix) . . . . . 42

Appendix II (Inventory Database Reports and Queries used to Support Sample

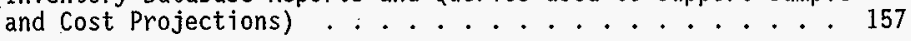

This package consists of pages 1 through 176.

TRADEMARKS:

Hastelloy is a Trademark of Stellite Rod Division Stoody Deloro Stellite Inc.

Teflon is a Trademark of E.I. du Pont de Nemours \& Co.

Kynar is a Registered Trademark of Elf Atochem of North America Inc.

Paradox is a Trademark of Borland International Inc.

Access is a Trademark of Microsoft 


\section{HNF-1041 PEV. O}

\section{Executive Summary}

The Hanford Tank Waste Remediation Systems (TWRS) Characterization Program is responsible for coordinating the sampling and analysis of the 177 large underground storage tanks at the Hanford site. The 222-S laboratory has been the primary laboratory for chemical analysis of this highly-radioactive material and has been accumulating these samples for many years. As part of the Fiscal Year 1998 laboratory work scope, the 222-S laboratory has performed a formal physical inventory of all tank characterization samples which are currently being stored. In addition, an updated inventory/archive program has been designed. This program defines sample storage, retention, consolidation, maintenance, and disposition activities which will ensure that the sample integrity is preserved to the greatest practical extent. In addition, the new program provides for continued availability of waste material in a form which will be useful for future bench-scale studies. Finally, when the samples have exceeded their useful lifetime, the program provides for sample disposition from the laboratory in a controlled, safe and environmentally compliant manner.

The 222-S laboratory maintains custody over samples of tank waste material which have been shipped to the laboratory for chemical analysis. The storage of these samples currently requires an entire hotcell, fully dedicated to sample archive storage, and is rapidly encroaching on additional hotcell space. As additional samples are received, they are beginning to limit the 222-S laboratory $11 \mathrm{~A}$ hotcell utility for other activities such as sample extrusion and subsampling. The 222-S laboratory tracks the number of sample containers and the mass of each sample through an internal database which has recently been verified and updated via a physical inventory.

The archive sample inventory, as of February 1998 , includes $203.7 \mathrm{Kg}$ of material in 2883 jars from 113 tanks. Of these samples $38 \%$ are sludge samples, $33 \%$ are salt cake samples, $18 \%$ are drainable liquid (supernate) samples, and the remaining $11 \%$ are grab samples, liner liquid, field blank, and miscellaneous samples. This inventory includes larger samples, most of which are being stored in the 222-S laboratory $11 \mathrm{~A}$ Hotcells. It does not include the small subsamples generated for laboratory analysis and stored in the 222-S laboratory Room 2B.

The inventory/archive program described in this document is substantially an implementation of the guidance described in HNF-SD-WM-TRD-006, Technical Basis for Disposition of Tank Waste Samples in Archive (Adams, M.R., et af), March 1997. The program calls for generation of "parent" subsamples and "laboratory" subsamples at the time of segment or auger extrusion (or grab sample breakdown). The parent subsamples are normally retained in the $11 \mathrm{~A}$ hotcells but some are retained in the $2 \mathrm{~B}$ laboratory storage area. The "laboratory" samples are delivered to the rest of the 222-S laboratory for further 
samples), as well as all of the small samples, digests and washes (altered samples) which are derived from them will be retained for 90-days past the publication date of the final analytical report. At that time they will be returned to Tank Farms. Any parent subsamples containing less than $5 \mathrm{~g}$ of material will also be dispositioned after 90 days as will samples from tanks which are actively receiving or transferring waste (non-static tanks).

During the first year after publication of the laboratory report, the TWRS Characterization Program, through its Sample Archive Disposal Team (SADT) will canvass the site programs, looking for interest in any of the specific parent samples in storage at the 222-S laboratory. If, after one year, no interest is identified, the SADT will consider the samples no-longer necessary. The laboratory will retain all samples for which a programmatic need has been identified. In addition, the laboratory may consolidate the SADT-disposable samples into core and matrix composites if a generic program need is identified. This consolidation serves two purposes. The first purpose is to increase the volume to minimize moisture-loss and the second is to reduce the overall number of containers to reduce maintenance costs. This consolidation also provides a ready source of the large volume samples which will be attractive to future bench-scale testing.

These consolidated samples will be repackaged for long-term storage and will be retained until the sample is shipped to an external user, the samples deteriorate to a non-useful state, there is a need to reduce laboratory inventory for space or safety-basis reasons, or the programmatic need lapses.

Inventory projections were made based upon the, TWRS Sampling Schedule Rev $5.3(3 / 11 / 98)$. Maximum number of jars in inventory runs from approximately 3500 to over 6000 for the three scenarios. In addition, since the TWRS programs have indicated the need to save more than just $60-100 \mathrm{~g}$ of each segment, the small archive jars will be replaced by $125 \mathrm{~mL}$ and $250 \mathrm{~mL}$ jars as time goes on. The current $11 \mathrm{~A}$ hotcells storage arrangement would not be able to accommodate all 6000 samples in the worst-case projection. If the cell storage space were re-designed to accommodate the larger sample, the $11 \mathrm{~A}$ space should be sufficient. Overflow capacity is available in the other 222-S laboratory hotcells.

Maintenance of the existing inventory includes repackaging after two years in the glass containers. This is necessary due to the chemical- and radiation-induced deterioration of the package materials. Maintenance costs are estimated at $\$ 170,000$ in 1998 , decreasing to $\$ 2,000$ in 2010 (1998 dollars) as sampling rates decrease. There is a wide variation in projected repackaging costs for the first four years. These costs could be managed in a way which reduces first-year costs to approximately $\$ 120,000$. 


\section{MNF-1641 REV. 0}

\section{Introduction}

The Hanford site stores residual processing waste in 177 underground storage tanks. The material being stored in these tanks has been sampled over the years for characterization to address current safety issues and future retrieval and processing activities. Due to the high radioactivity of the tank waste samples, all chemical analysis has been conducted at the Hanford high-level laboratories (222-S and 325). The 325 laboratory tank waste characterization program was phased out in 1996 and since then all waste tank characterization has been performed at the 222-S laboratory. Tank characterization activities are expected to continue for many years as the waste in the tanks is stored, retrieved and, ultimately, processed for disposition from 222-S laboratory.

Recovering samples from these massive storage tanks is a complicated and costly activity. It involves physical, chemical, and radiological hazards to workers. For these reasons the Tank Waste Remediation System (TWRS) organization is under great pressure to retain all samples for potential future use. It is the hope that the availability of this material will obviate the need to re-sample tanks in some cases.

The definition of the sample long-term storage needs have been the subject of many studies in recent years (CBM-94-002 (McVey, 1994); WHC-SD-W079-ES001 (McVey, 1994); Letter From D. R. Bratzel to S. M. Joyce, June 23, 1994). The nature of sample packaging, storage location, inventory program, retention time and disposition approaches have been studied, but not conclusively determined. This is due, in part, to changes in sampling rate, budgets, and the availability of a large volume of hot-cell space at the 222-S laboratory. However, within the last year, archive samples have exceeded the storage bin capacity allocated and are currently being stored on cell floors, makeshift baskets and are encroaching on active areas of the 222-S laboratory $11 \mathrm{~A}$ hotcells.

Archive requirements were described in detail in a Characterization Program letter 7E720-94-125, D. R. Bratzel to S. M. Joyce, dated June 23, 1994. The letter recommends a six-month waiting period from the date of a TCR being published. After six months, the core composite would be kept if it contains greater than $60 \mathrm{~g}$ of material and all of the samples from the core would be offered to potentially interested programs. If no interest is expressed, all but the composite would be dispositioned. The most likely disposition is a return of the material to Tank Farms through the 219-S Waste Handling Facility. If there was insufficient material in the composite sample, the laboratory would prepare a composite from the archived segments and store in a $100 \mathrm{~mL}$ jar. The composites were then to be archived for 5 years. Grab and auger samples would be kept for six months after the TCR was published. If there is no interest in these samples they will be dispositioned. No grab or auger samples were to be kept for longterm archive. 


\section{MANF-1041 PEU. O}

processing and chemical This guidance has been recently superceded by Adams, et al (HNF-SD-WM-TRD-006). The idea of compositing was abandoned and all sample material will be retained, not just a $60 \mathrm{gm}$ composite. The waiting times and ultimate storage times were also modified.

As part of the Waste Characterization FY 1998 Multi-Year Work Plan, through WBS 1.1.1.02.11, the 222-S laboratory is to undertake several activities associated with sample storage and disposition, given the updated guidance. The storage and disposition activities include:

- performing a physical inventory of all samples at the 222-S laboratory

- maintenance and surveillance of samples currently stored at the 222S laboratory,

- evaluation of potential sample storage areas,

- Making ready sample storage areas,

- Packaging and shipping samples for storage or disposition,

- Providing continuing storage and/or disposition of samples, and

- Providing for ultimate sample disposition and cleanout of storage areas.

This document describes the results of the physical inventory, evaluation of storage and maintenance requirements and development of an inventory program for accomplishing all of the above-mentioned items. 


\section{Proposed Archival Program}

\section{Process Description}

The proposed inventory program is based substantially upon guidance published in HNF-SD-WM-TRD-006 Technical Basis for Disposition of Tank Waste Samples in Archive (Adams, M.R., et a), March 1997. The major components can be summarized as follows:

- Tank segments are broken down and analyzed as per a Tank Sampling and Analysis Plan (TSAP),

- A 90-day clock begins upon completion of the laboratory analytical report.

- During the 90 days, all subsamples must be available for re-analysis or additional characterization.

- After the 90-day period, small samples $(<5 \mathrm{~g})$, daughter samples, altered samples (digestates) and non-static tank (a tank which has had material added or removed since the sampling event) sample material can be dispositioned.

- Up to 1 year from the publication of the laboratory report, the Sample Archive Disposal Team (SADT) will canvass the Hanford Site programs, looking for indication of interest in retention of specific samples.

- If such interest is provided, the requested samples will be retained up to 5 years.

- Any other samples will be dispositioned after the 1-year period.

Figure 1 shows the process as a diagram.

The program proposed in this document takes the main elements of the guidance from HNF-SD-WM-TRD-006 and introduces the concept of consolidating like samples at the one-year period (instead of immediate return to Tank Farms), and indefinite storage of these larger composites. Instead of triggering the disposition sequence at the 1-year mark, the recommended program would retain large-volume composite samples for projected beneficial use in bench-scale studies. Decisions to disposition this material may come at any time after the characterization program has indicated no further interest. This concept can only 173-303-071(3)(l)), allow sample archiving only where 


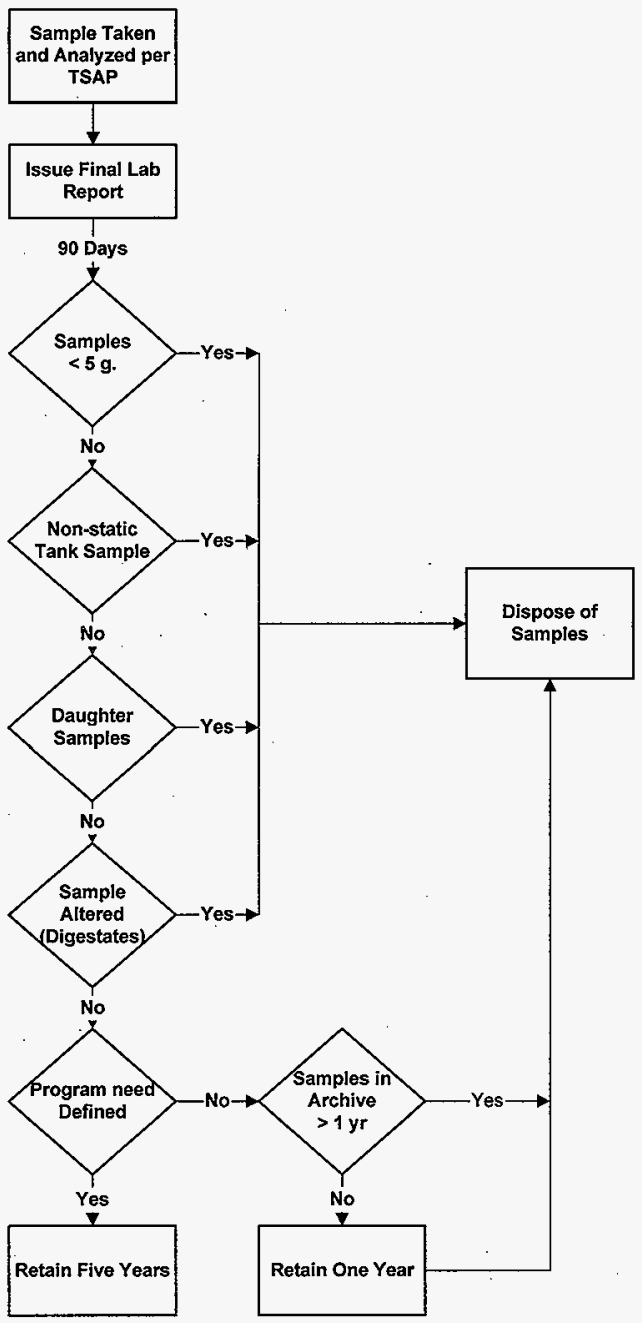




\section{HNF-1041 FEV. 0}

there is a specific purpose for retention identified. Treatability study treated material may be archived for future evaluation for up to 5 years from initial receipt of the treatability sample at the lab (WAC 173-303-071-(3)(s).

Figure 2 depicts the new recommended program.

\section{Program detail description}

The first modification of current practice is to end the practice of generating a separate "archive" sample. The "parent" jar will also be an archive jar. This will enhance sample integrity by increasing individual sample volume and simplify storage and maintenance by decreasing the overall number of containers in storage. There should also be a component of the inventory program to repackage the parent jar into the smallest jar which will contain the remaining material.

It is possible that repeated subsampling from a parent jar may increase the likelihood of cross-contamination or sample degradation which could be avoided if two jars are created. This potential must be weighed against the benefit of storing the samples in fewer, larger-volume containers. Given that repeated subsampling is, in fact, very limited and that every sample will benefit from larger volume storage, it seems that the benefits favor retaining only one parent jar.

Upon publication of the formal laboratory report on the analysis of a sample, a 90-day waiting period will commence. During this period the laboratory must maintain the ability to quickly return to a specific subsample in order to confirm anomalous results or answer additional characterization questions. Although Memoranda of Understanding (MOU) between TWRS Characterization and 222S Laboratory (e.g. HNF-SD-WM-SD-023, Fiscal Year 1997 Memorandum of Understanding for the TWRS Characterization Project), have traditionally stated that laboratory subsamples could be dispositioned immediately upon publication of the laboratory report, the 90-day waiting period identified in HNF-SD-WMTRD-006 seems prudent. If laboratory space is insufficient to maintain samples for the entire 90-day period, special permission to disposition specific samples may be requested from the Characterization Program.

At the 90-day mark, any subsamples, digestates, liner liquid, and subsamples containing $<5 \mathrm{~g}$ will be identified as "dispositionable." These will then be dispositioned in a controlled manner, consistent with appropriate RCRA regulations, ALARA, and laboratory operational conditions. Non-static tanks, where identified, will generally be retained for consolidation unless there is clearly no future use (i.e. the matrix or contents are too unique and no longer exist so that there would be no use even as "example" waste). 
Figure 2

MNF-1641 REV. 0

Revised Process for Archiving New Samples

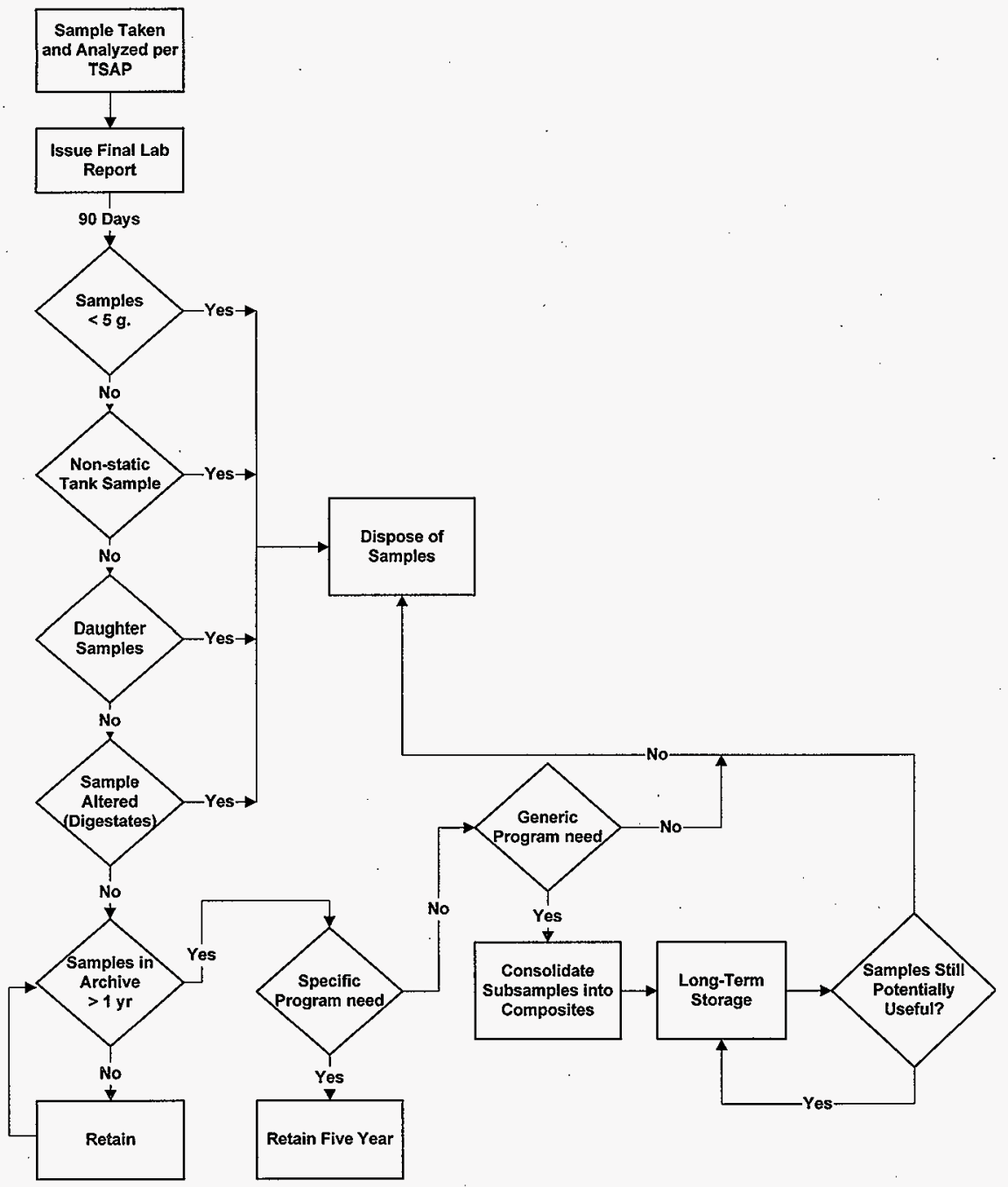




\section{MANF-1641 REV. 0}

Between the issuance of the laboratory report and one-year afterward, the SADT will conduct the administrative process for identifying programmatic interest in individual samples as described in HNF-SD-WM-TRD-006.

At the one-year mark, samples of specific programmatic interest will be identified for archive. The rest of the samples may be consolidated into a limited number of core composites if a programmatic need is identified and documented. Laboratory chemists will work with Characterization Program technical counterparts and develop a tank-specific consolidation scheme. It is anticipated that, in most cases, the scheme will be to combine samples from the same core, same matrix into core:matrix composites. In some cases, all samples from a core will be combined. Other compositing/consolidation schemes may be identified as most appropriate for that tank material. If a tank has only small amounts of material with limited potential future use, or if it contains an excess of a very common material, full or partial disposition may be the appropriate action.

Between year-one and year-two, the consolidation scheme will be accomplished. One. year is allocated to the task of designing and accomplishing the consolidation/disposition plan. This is to give consideration to ongoing activities in the laboratory, workload of the project coordinators and Characterization Program counterparts, and operational schedule and status of the facility and, in particular, the 219-S Waste Handling Facility and cross-site waste liquid transfer line.

The resulting composites will be packaged in stainless steel, Hastelloy, or borosilicate glass containers for long-term archiving. Consolidated samples will remain in long-term storage until they are used at the 222-S laboratory, shipped to an off-site user or dispositioned by the facility.

There is currently a substantial backlog of samples in the 222-S laboratory inventory. Implementation of the archive/disposition plan will involve an initial set of activities in 1998 to consolidate the backlog and set-up the program. These initial implementation activities are described later in this report. 


\section{Physical Inventory}

The storage of tank samples currently requires an entire hotcell, fully dedicated to sample archive storage. As additional samples are received, they are beginning to limit the 222-S laboratory 11A hotcell utility for other activities such as sample extrusion and subsampling. The 222-S laboratory tracks the number of sample containers and the mass of each sample through an internal database. However, precise reconciliation against a physical inventory has not happened until this activity.

A full physical inventory of all samples currently stored in the 222-S laboratory $11 \mathrm{~A}$ hot cells was completed in March 1998. The inventory process was the most-involved, and provided the most accurate data on sample inventory since the lab began receiving tank-waste samples nearly 10 years ago. Each sample jar was removed from its storage compartment, individually weighed, and its matrix verified against extrusion logs. New database fields were added for net weight of sample and matrix of sample. This enabled the database to now contain actual rather than estimated sample net weights and an indication of the sample matrix (sludge, saltcake, supernate, liner liquid, etc).

The 222-S laboratory archive sample inventory, as of March 1998, includes $203.7 \mathrm{Kg}$ of material in 2883 jars from 113 tanks. Of these samples $38 \%$ are sludge samples, $33 \%$ are salt cake samples, $18 \%$ are drainable liquid (supernate) samples, and the remaining $11 \%$ are grab samples, liner liquid, field blank, and other miscellaneous samples. Tables 1-3 and Figure 3 show various summaries of the inventory.

The inventory revealed 40 samples ( $1 \%$ of the total) which existed but were not in the database, and 120 samples ( $4 \%$ of the total) for which the database indicated some remaining material, but which had actually been depleted. Approximately 20 jars $(0.7 \%)$ were broken during the inventory process and had to be repackaged.

Appendix $\mathrm{l}$ is a printout of the 222-S laboratory archive database as validated by the March 1998 physical inventory. This is the portion of the sample inventory which exists in the 11A hotcells. Additional tank samples are stored in room $2 \mathrm{E}$ and $2 \mathrm{~B}$. There are currently 26 grab liquid samples being stored in Room $2 \mathrm{~B}$ and 53 miscellaneous samples from 15 tanks in 2E. Table 4 details these samples. 
Table 1:

\section{2-S Physical Inventory - 1998 \\ Archive Sample Mass per Tank}

\begin{tabular}{|c|c|c|c|c|c|}
\hline Tank & mass $(g)$ & Tank & mass $(g)$ & Tank & $\operatorname{mass}(\mathrm{g})$ \\
\hline A-101 & 7655 & BY-101 & 255 & SY-102 & 1452 \\
\hline AN-101 & 409 & $B Y-102$ & 943 & SY-103 & 2051 \\
\hline AN-102 & 550 & $B Y-103$ & 109 & $T-104$ & 134 \\
\hline AN-103 & 9283 & BY-104 & 2157 & $T-105$ & 742 \\
\hline AN-104 & 10555 & $B Y-105$ & 754 & $T-106$ & 42 \\
\hline AN-105 & 5025 & BY-106 & 5418 & $T-107$ & 9 \\
\hline AN-106 & 375 & BY-107 & 2562 & $T-108$ & 46 \\
\hline AN-107 & 760 & BY-108 & 2261 & $T-109$ & 214 \\
\hline AP-101 & 470 & BY-109 & 2859 & $\mathrm{~T}-110$ & 3856 \\
\hline AP-104 & 141 & BY.110 & 5105 & $T-112$ & 904 \\
\hline AP-105 & 2432 & $B Y-111$ & 1506 & $T-201$ & 1364 \\
\hline AP-106 & 138 & BY-112 & 1604 & $\mathrm{~T}-202$ & 1076 \\
\hline AP-108 & 96 & C-101 & 230 & $T-203$ & 2345 \\
\hline$A W-101$ & 5762 & C-102 & 26 & $T-204$ & 2723 \\
\hline AW-102 & 375 & $\mathrm{C}-103$ & 1214 & TX-107 & 14 \\
\hline AW-103 & 4254 & C-104 & 1873 & $\mathrm{TX}-244$ & 278 \\
\hline AW-104 & 3656 & C-105 & 285 & $T \gamma-104$ & $\frac{\pi}{93}$ \\
\hline$A W-105$ & 4039 & C-107 & 1390 & TY-106 & 106 \\
\hline AW-106 & 773 & C-108 & 293 & U-101 & 327 \\
\hline$A X-101$ & 4824 & C-111 & 217 & U-102 & 3265 \\
\hline$A X-102$ & 149 & C-201 & 34 & U-103 & 4361 \\
\hline$A X-103$ & 692 & C-202 & 75 & U-105 & 3018 \\
\hline$A x-104$ & 238 & C-203 & 802 & U-106 & 2282 \\
\hline$A Y-101$ & 975 & C-204 & 157 & U-107 & 2113 \\
\hline$A Y-102$ & 364 & S-101 & 6031 & $\mathrm{U}-108$ & 7380 \\
\hline B-101 & 676 & s-102 & 3283 & U-109 & 4250 \\
\hline B-102 & 3 & S-104 & 150 & U-112 & 338 \\
\hline B-104 & 3531 & S-106 & 3406 & U.201 & 1203 \\
\hline B-106 & 859 & S-107 & 4850 & U-202 & 1202 \\
\hline B-107 & 837 & S-109 & 1612 & U-203 & 257 \\
\hline B-108 & 482 & $S-110$ & 1484 & U-204 & 458 \\
\hline B-109 & 643 & S-111 & 4645 & & \\
\hline B-203 & 3838 & S-151 & 139 & Special & 695 \\
\hline B-204 & 6126 & $5 x-101$ & 4501 & $219-5$ & 40 \\
\hline BX-101 & 79 & SX-103 & 274 & 972-SLY & 14 \\
\hline$B X-103$ & 670 & SX-104 & 456 & Tk-101 & 452 \\
\hline $8 X-105$ & 88 & $5 x-106$ & 4725 & & \\
\hline$B \times-106$ & 193 & $5 x-108$ & 67 & & \\
\hline BX-109 & 2134 & $5 x-113$ & 121 & & \\
\hline$B X-110$ & 1583 & & & & \\
\hline $\mathrm{BX}-111$ & 667 & & & & \\
\hline$B X-112$ & 305 & & & total & 203681 \\
\hline
\end{tabular}


Table 2:

\section{2-S Physical Inventory - 1998 \\ Archive Sample Mass per Tank \\ Rank Ordered}

\begin{tabular}{|c|c|c|c|c|c|}
\hline Tank & mass $(g)$ & Tank & $\operatorname{mass}(\mathrm{g})$ & Tank & $\operatorname{mass}(g)$ \\
\hline AN-104 & 10555 & S-109 & 1612 & U-101 & 327 \\
\hline AN-103 & 9283 & $\mathrm{BY}-112$ & 1604 & $B X-112$ & 305 \\
\hline$A-101$ & 7655 & $B X-110$ & 1583 & C-108 & 293 \\
\hline U-108 & 7380 & $B Y-111$ & 1506 & $C-105$ & 285 \\
\hline B-204 & 6126 & S.110 & 1484 & TX-244 & 278 \\
\hline S-101 & 6031 & SY-102 & 1452 & $5 X-103$ & 274 \\
\hline AW-101 & 5762 & C-107 & 1390 & U-203 & 257 \\
\hline BY-106 & 5418 & $T-201$ & 1364 & $B Y-101$ & 255 \\
\hline $\mathrm{BY}-110$ & 5105 & $\mathrm{C}-103$ & 1214 & AX-104 & 238 \\
\hline AN-105 & 5025 & U-201 & 1203 & $\mathrm{C}-101$ & 230 \\
\hline S-107 & 4850 & $\mathrm{U}-202$ & 1202 & C-111 & 217 \\
\hline$A X-101$ & 4824 & T-202 & 1076 & $T-109$ & 214 \\
\hline $5 x-106$ & 4725 & $A Y-101$ & 975 & $B X-106$ & 193 \\
\hline S-111 & 4645 & $B Y-102$ & 943 & C-204 & 157 \\
\hline $5 x-101$ & 4501 & $T-112$ & 904 & S-104 & 150 \\
\hline $\mathrm{U}-103$ & 4361 & B-106 & 859 & $A X-102$ & 149 \\
\hline AW-103 & 4254 & B-107 & 837 & AP-104 & 141 \\
\hline U-109 & 4250 & C-203 & 802 & S-151 & 139 \\
\hline AW-105 & 4039 & $A W-106$ & 773 & AP-106 & 138 \\
\hline$T-110$ & 3856 & AN-107 & 760 & $T-104$ & 134 \\
\hline B-203 & 3838 & BY-105 & 754 & $5 X-113$ & 121 \\
\hline$A W-104$ & 3656 & $T-105$ & 742 & $B Y-103$ & 109 \\
\hline B-104 & 3531 & Special & 695 & TY-106 & 106 \\
\hline $5-106$ & 3406 & AX-103 & 692 & AP-108 & 96 \\
\hline S-102 & 3283 & B-101 & 676 & TY-104 & 93 \\
\hline $\mathrm{U}-102$ & 3265 & $\mathrm{BX}-103$ & 670 & BX-105 & 88 \\
\hline $\mathrm{U}-105$ & 3018 & $B X-111$ & 667 & $B X-101$ & 79 \\
\hline BY-109 & 2859 & B-109 & 643 & C-202 & 75 \\
\hline$T-204$ & 2723 & AN-102 & 550 & $5 x-108$ & 67 \\
\hline$B Y-107$ & 2562 & B-108 & 482 & $T-108$ & 46 \\
\hline AP-105 & 2432 & AP-101 & 470 & $\mathrm{~T}-106$ & 42 \\
\hline T-203 & 2345 & U-204 & 458 & $219-5$ & 40 \\
\hline $\mathrm{U}-106$ & 2282 & $5 x-104$ & 458 & C-201 & 34 \\
\hline$B Y-108$ & 2261 & Tk-101 & 452 & $C-102$ & 26 \\
\hline$B Y-104$ & 2157 & AN-101 & 409 & 972-SLY & 14 \\
\hline BX-109 & 2134 & AN-106 & 375 & TX-107 & 14 \\
\hline U-107 & 2113 & $A W-102$ & 375 & $\mathrm{~T}-107$ & 9 \\
\hline$S Y-103$ & 2051 & AY-102 & 364 & $B-102$ & 3 \\
\hline $\mathrm{C}-104$ & 1873 & $\mathrm{U}-112$ & 338 & & \\
\hline
\end{tabular}




\section{2-S Physical Inventory - 1998 Summary by Matrix}

\begin{tabular}{|l|r|r|}
\hline Matrix & \# of jars & total wt (g) \\
\hline \hline Sludge & 1094 & 71131 \\
\hline Salt Cake & 962 & 63628 \\
\hline Drainable liquid & 514 & 46697 \\
\hline Grab liquid & 171 & 11694 \\
\hline Field Blank & 86 & 7545 \\
\hline Liner Liquid & 50 & 2613 \\
\hline unknown & 2 & 227 \\
\hline Grab solid & 2 & 77 \\
\hline Cord & 1 & 64 \\
\hline Organic (BY-108) & 1 & 8 \\
\hline Total & 2883 & 203684 \\
\hline
\end{tabular}


Figure 3:

\section{2-S Physical Inventory - 1998 Summary by Matrix}
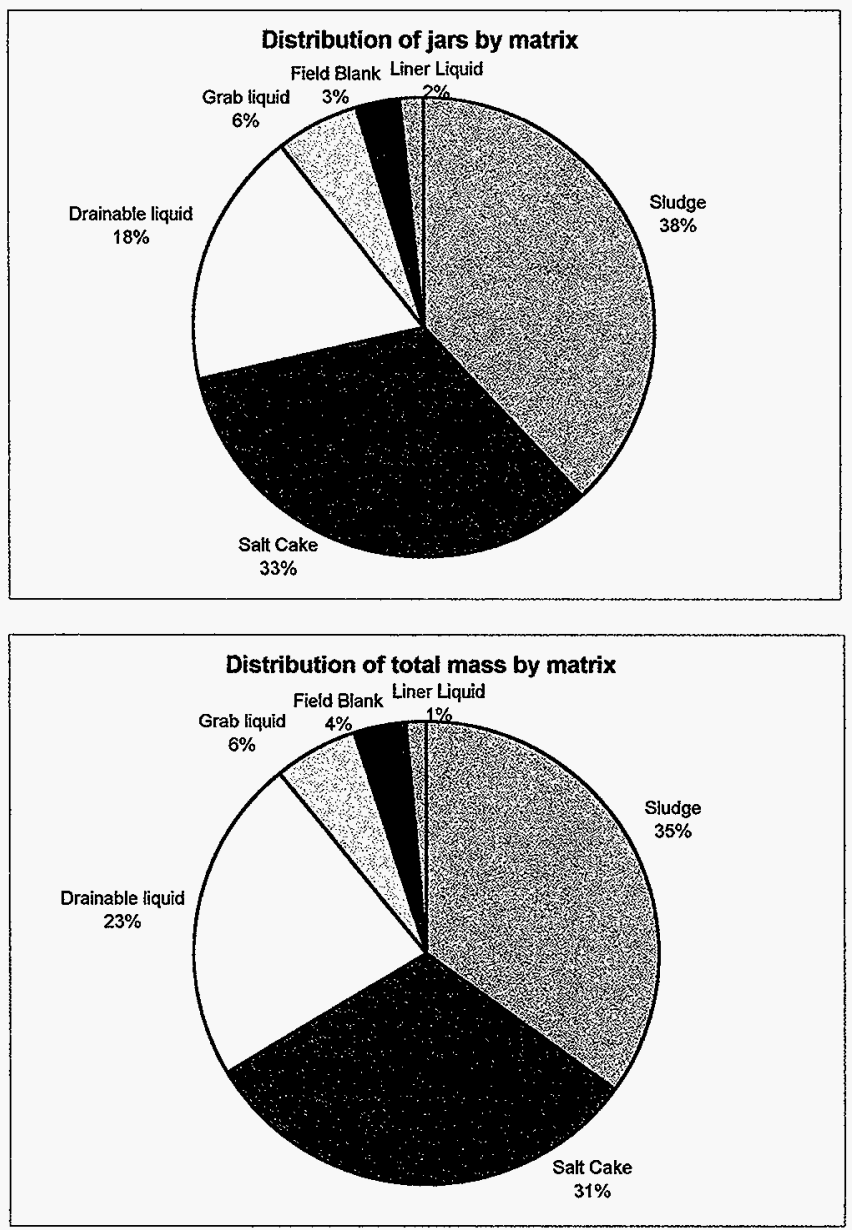
Table 4: MNF-1641 REV. 0

\section{Miscellaneous Tank Characterization Samples Currently Stored in 222-S, Rooms 2B and 2E}

\begin{tabular}{|l|r|c|}
\hline Tank & Samples & Matrix \\
\hline \hline Room 2B & & \\
\hline AP-104 & 8 & grabliquid \\
\hline AP-106 & 3 & grab liquid \\
\hline AP-107 & 3 & grab liquid \\
\hline AP-108 & 5 & grab liquid \\
\hline AY-102 & 4 & grab liquid \\
\hline C-106 & 3 & grab liquid \\
\hline & & \\
Room 2E & & \\
\hline AN-102 & 2 & $? ? ?$ \\
\hline AN-106 & 2 & $? ? ?$ \\
\hline AN-107 & 3 & $? ? ?$ \\
\hline AP-106 & 1 & $? ? ?$ \\
\hline AY-102 & 5 & $? ? ?$ \\
\hline B-202 & 13 & $? ? ?$ \\
\hline BY-105 & 4 & $? ? ?$ \\
\hline BY-110 & 1 & $? ? ?$ \\
\hline S-101 & 1 & $? ? ?$ \\
\hline SY-102 & 3 & $? ? ?$ \\
\hline T-103 & 2 & $? ? ?$ \\
\hline T-104 & 10 & $? ? ?$ \\
\hline T-105 & 1 & $? ? ?$ \\
\hline T-107 & 4 & $? ? ?$ \\
\hline TX-104 & 1 & $? ? ?$ \\
\hline
\end{tabular}




\section{HNF-1641 REV. 0}

\section{Containers}

The current tank waste package is a borosilicate jar (various volumes from $40 \mathrm{~mL}$ to $250 \mathrm{~mL}$ ) with a phenolic screw-cap and Teflon liner. This package represents a balance of cost, availability, inertness, and operational ease.

In 1993, the Analytical Evaluation and Reporting organization generated WHCSD-WM-TI-569 Tank Core Sample Archiving Container Fabrication Material. This report acknowledged the current use of borosilicate glass but gathered scientists, engineers and materials specialists to discuss the potential for sample contamination and investigate other potential packaging materials. Their consensus was that the borosilicate glass may leach boron or silicon into the waste samples and that the high $\mathrm{pH}$ of the waste may etch or degrade the glass over time.

In addition to borosilicate glass, the group evaluated Hastelloy, Stainless Steel, Polypropylene, Kynar, and Teflon. The group recommended Hastelloy as the most inert packaging material and suggested that Hastelloy containers could be acquired at a cost of $\$ 30 /$ container.

In the intervening years, significant operating experience has been gained in the use of the borosilicate glass container. It is cheap (roughly $\$ 3 /$ container), available off-the-shelf in prewashed condition, is the USEPA container of choice for most solid samples, is initially transparent, and available in wide-mouth geometries enabling greater operational ease in filling.

On the down-side, there may be some trace-level boron and silicon introduced into a high-pH sample, but the boron and silicon content of the samples is not of very high importance and the jars are cheap, readily available, and disposable. The radioactivity of the samples deteriorates the polymer cap and liner materials over time. This results in brittleness and potential cap failure due to cracking. It also complicates retrieval activities in that manipulation of the cap generally involves cap breakage. Sometimes the cap or liner fragments wind up in the sample jar, requiring difficult removal activities which challenge remote manipulator operators.

Practical experience suggests that 2-3 years is the maximum lifetime for the phenolic caps and the borosilicate glass, (R. Akita personal comm.). Therefore the samples must be either repackaged or dispositioned of within the first 3 years from extrusion.

Another proposed option was to use the same borosilicate glass container, in which a stainless steel or Hastelloy liner or beaker is inserted. However, unless the liner is machined to fit snugly into the jar, the increase in headspace would be 
a detriment to the package, allowing greater moisture loss. In addition, the addition of the liner would not appreciably reduce the radiolytic effect on the glass package.

The new inventory program calls for movement of samples from short-term to long-term storage at 1-3 years from the data of the laboratory analytical report on the samples. Using borosilicate glass containers for this first 1-3 years and then shifting to either a new borosilicate package for another 2-3 years, or stainless steel/hastelloy for long-term storage (indefinite length of time) represents a reasonable trade-off between sample integrity, cost, and ease-of-operation.

As of the preparation of this document, no source of Hastelloy containers has been identified. Stainless steel containers are available through Cole-Parmer Instrument $\mathrm{Co}$. The smallest available size listed is $\mathrm{L}$ at a cost of $\$ 660 /$ container. This may be useful for future large-volume storage, and some long-term storage samples may be that large, when consolidated. However, smaller containers are still needed. In addition, the compression seal for the stainless steel container may not be easy to close using manipulators, and the soft-polymer washer may be prone to radiolysis. There will be a need to retain some large-volume samples. This stainless-steel package should be evaluated and modified for hot-cell use. 


\section{Storage Needs and Current Readiness}

Two engineering studies of the 222-S laboratory archive storage space needs at the 222-S laboratory have been conducted (CBM-94-002, WHC-SD-W079-ES001).

The CBM-94-002 document suggested the $2 \mathrm{E}$ room as a potential location for up to $40 \%$ of the storage containers. The attractiveness of this as an option has decreased in the last few years because of changes in the storage needs, (larger volumes now being archived), and also the impact to $222-\mathrm{S}$ laboratory operations.

Room $2 E$ is a small sample storage area which is currently posted as a Radiological Contamination Area (RCA). Utilization of $2 E$ as a archive storage area for tank samples would increase the likelihood of the status being changed to a High Radiation Area (HRA) on a frequent, if not continuous, basis. This status change would increase security and surveillance requirements for $2 \mathrm{E}$. Also, the assumptions of sample size $(40 \mathrm{~mL})$ have changed and are now 100 $250 \mathrm{~mL}$ or more for long-term storage. This would limit the number of samples which could potentially be archived within $2 \mathrm{E}$ without generating unacceptably high radiation fields. It would also require additional care in shielding and sample physical arrangement within the room. Even if the shielding, sample arrangement, and operational activities could be performed, $2 \mathrm{E}$ would still require more additional operational attention than archival in the hotcells.

CBM-94-002 also recommended the purchase of portable buildings for storage of sample material $(\$ 100,000$ est.). McVey acknowledged that a safety review would be required, also regulatory permitting and that this would only provide temporary storage until a permanent building $(\$ 720,000)$ would be constructed. He justified the recommendation by including considerations of off-site sample return, 2800 core segment samples and up to 95 core-segment-casks. Severe revision of these estimates, budget constraints and increased scrutiny on safety and regulatory compliance have made this recommendation moot.

WHC-SD-W079-ES-001 describes an engineering study which concludes that the 222-S laboratory has enough archive space to store approximately $360040 \mathrm{~mL}$ jars and $1080100 \mathrm{~mL}$ jars. This was determined to be sufficient for the sample projections current in 1994. Archive facility options considered included:

- do nothing,

- build a new permanent facility,

- modify existing 222-S and 324/325 facilities,

- use closed facilities such as 202-S (REDOX), FMEF, or WESF,

- add-on to existing facilities and

- re-sampling vs archiving. 
The preferred alternative was to modify existing facilities by purchase of storage racks and identify all potential storage locations. There was a concern that this option would prevent the hotcells from being available for other scopes.

Nevertheless it was, by far, the least-cost alternative. The most recent evaluation of potential storage identified a cost of over $\$ 1.5$ million to store samples at the Central Waste Complex (Hall 1996)

On the basis of this recommendation, and the immediate need for additional sample storage, the 222-S laboratory designed and fabricated additional storage trays for installation in the 11A2 Cell. Each of these new trays can hold up to 12 $60 \mathrm{~mL}$ jars or five larger ( 125 or $250 \mathrm{~mL}$ ) jars. The storage system in $11 \mathrm{~A} 2$ requires installation of wall racks which will hold eight trays each. It is unknown how many racks will be able to be installed, but estimates range from 5 to 7 .

There are also 32 floor bins (baskets) available. Each of these baskets will hold up to $19250 \mathrm{~mL}$ jars or $28125 \mathrm{~mL}$ jars. These baskets are stackable and moveable. The total available storage in $11 \mathrm{~A}$ is shown in Table 5.

\section{Table 5: Current Sample Storage in the 11A Cells}

\begin{tabular}{|l|l|l|l|l|}
\hline Location & $\begin{array}{l}\text { Capacity available } \\
\text { (theo.) }\end{array}$ & Capacity used & $\begin{array}{l}\text { Capacity } \\
\text { remaining } \\
\text { (theo.) }\end{array}$ & $\begin{array}{l}\text { Capacity } \\
\text { remaining } \\
\text { (act.) }\end{array}$ \\
\hline $\begin{array}{l}11 \mathrm{~A} 1 \\
\text { wall bins }\end{array}$ & $225040 / 60 \mathrm{~mL}$ & $205040 / 60$ & $20040 / 60 \mathrm{~mL}$ & none \\
\hline $\begin{array}{l}11 \mathrm{~A} 1 \\
\text { floor baskets }\end{array}$ & $\begin{array}{l}896125 \mathrm{~mL} \text { or } \\
608250 \mathrm{~mL}\end{array}$ & $\begin{array}{l}164125 \mathrm{~mL} \mathrm{\&} \\
425250 \mathrm{~mL}\end{array}$ & $69250 \mathrm{~mL}$ & none \\
\hline $\begin{array}{l}11 \mathrm{~A} 1 \\
\text { floor tubs }\end{array}$ & $45125 \mathrm{~mL}$ & $45125 \mathrm{~mL}$ & none & none \\
\hline $\begin{array}{l}11 \mathrm{~A} 1 \\
\text { floor }\end{array}$ & & $199250 \mathrm{~mL}$ & & none \\
\hline $\begin{array}{l}11 \mathrm{~A} 2 \\
\text { anticipated }\end{array}$ & $200-280125 / 250 \mathrm{~mL}$ & none & $200-280125 / 250 \mathrm{~mL}$ & $100 \%$ \\
\hline TOTAL & $3103-3471$ jars & 2883 jars & 270-549 jars & $10-20 \%$ \\
\hline
\end{tabular}

* theoretical ultimate capacity, assuming optimal packing density.

${ }^{* *}$ actual remaining capacity as observed. 


\section{Sample Projections}

The TWRS Sample Schedule Rev $5.3(3 / 11 / 98)$ was used to project the number and size of sample jars from now through 2010 . Tables 6,7 , and 8 are year-byyear current projections of grab, push, and rotary samples to be generated.

Figure 5 shows the resulting 222-S laboratory inventories under three different retention scenarios.

\section{Scenario one - Complete retention}

The first scenario is that no sample jars are dispositioned, all jars currently in the inventory stay forever. Future jars are generated at an average rate of one 125$\mathrm{mL}$ jar per grab sample and three-250 $\mathrm{mL}$ jars per push or rotary segment. These jars remain in the 222-S laboratory inventory indefinitely. The result is a rapid increase to approximately 6000 jars in 2003 and only slowly increasing thereafter.

Secenario two - Keep all samples for 5 years

The second scenario assumes the generation rate as in scenario one. In addition, an assumption is made that all samples will be dispositioned via the 219-S Waste Handling Facility after five years from extrusion. This scenario shows a maximum inventory of roughly 4800 jars in 1999 , with a decrease thereafter.

Scenario three - Implementation of all initiatives

The third scenario assumes the completion of a comprehensive inventory repackaging effort in 1998. In addition the SADT is active in identification of programmatic need for each sample between year one and two after the laboratory report. Finally, remaining samples are dispositioned after reaching an age of five years.

The repackaging assumption includes:

- disposition of unneeded samples: Field blanks and liner liquid samples greater than one year old, samples $<5 \mathrm{~g}$.

- elimination of much of the smaller jars by eliminating the need for both archive and parent jars

- repackaging $125 \mathrm{~mL}$ and $250 \mathrm{~mL}$ jar contents into smaller jars where the mass is low enough. 
MNF-1641 REV. 0

Table 6:

TWRS Characterization Grab Sample Projections 1998 - 2008

GSHT -9 GSHU-4

GSDR-4 $\operatorname{GS} \times 2-4$ GSGI 4

GSHV-4

GSDF-4

GSGV-4

GSHY - 4

GSHW- 4

GSEB-4

GSDD-4

GSHZ-4

GSXD-4

GSDM- 4

GSDG-4

GSNW-4

GSFF-4

GSFM-4

GS72-4

GSGJ-4

GSGT-4

GS36-4

GS28-4

GS38-4

GSDI -4

GSGX-4

GSGX-4

GSXI-4

GSEA-4

$6 \mathrm{SXC}-4$

GSED-4

GSGB-4

GSDK-4

GSER - 4

GSX3-4

GS73-4

GSHF-4

GSHX-4

GSH3-4

GSHL -4

GSHN-4

GSHQ-4

GSEC -4

GSAX-4

GSX 4-4

GSHD-A

GSXE-4

GS97-4

GSGL-4

GSXE-4

GSAY - 4

GSBC-4

GSXG-4 GSEM- 4

GSGP-4

GSGR-4

GSGN-4

GSGO -4

GSEX -4
AP-104 Grab Sample (Caustic Addn) Blaak AP-108 Grab Sample (Compatability) Blaak C-201 Grab Sample - Meacham C-202 Grab Sample

TX-302-C Grab Sample Compatability - Doeler U-301-B Grab Sample Compatability - Sutey AW-106 Grab Sample (After 97-2) -Blaak -102 Grab Sample (After 97-2)- Blaak AX-102 Grab Sample Blaak S-302 Grab Sample Compatability - Sutey AN-102 Grab Sample 2 Pxivatization - Wiercers AX-102 Grab Sample (Meacham)

SX-102 Grab Sample Compatability - Doeler AP-103 Grab Sample - Elaak-Caustic Verification SX-115 Grab Sarple

AW-101 Grab Sample 2 - Privatization

AN-107 Grab Sample 2 Privatization

AW-102 Campaign 98-1 Grab Sample - Le

AD-106 Grab Sample - Corapatability - Blaak

AP-104 Grab Sample - Blazk -Caustic Verification AZ-101 Grab Sample

AZ-101 Grab Sample

A2-101 Grab Sample 4

AY-102 Grab Sample C-106 Retreival (NCE)

AY-102 Grab Sample C-106 Retreival (NCF)

AY-102 Grab Sample C-106 Retreival (NCF)

AP-107 Grab Sample - Blaak- Caustic Verification AY-102 Grab Sample C-106 Retreival (NCF)

AY-102 Grab Samole C-106 Retreival (NCF)

AY-102 Grabs Sample C-106 Retreival (NCF)

AY-102 Grab Sample C-106 Retreival (NCF)

AY-102 Grab Sample C-106 Retreival (NCF)

SY-102 Grab Sample - (99-1 Compatibility) Blaak

AY-102 Grab Sample C-106 Retreival (NCF)

AY-102 Grab Samole C-106 Retreival (NCF)

TBD Grab Sample - (99-1 Compatibility) Blaak

AY-102 Grab Sample C-106 Retreival (NCT)

AY-102 Grab Sample C-106 Retreival (NCF)

Z-361 Grab Sample Bogen (NCE)

AY-102 Grab Sample C-106 Retreival (NCE)

AY-102 Grab Sample C-106 Retreival (NCF)

AY-102 Grab Sample C-106 Retreival (NCF)

AY-102 Grab Sample C-106 Retreival (NCF)

1998 Total Grabs

TBD Grab Sample (99-1 Campaign) - Le

AN-106 Grab Sample Privatization

TBD Grab Sample (99-2 Compatability) - Blaak SY-102 Grab Sample (99-2 Compatability) - Blaak TBD Grab Samplo (99-2 Campaign) - te

Az-101 Grab Sample Certa

SY-102 Grab Sample (00-1 Compatibility) - Blaak TBD Grab Sample (00-1 Compatability) - Blaak

AP-101 Grab Sample Privatization

AW-104 Grab Sampie Privatization

1999 Total Grabs

TBD Grab Sample - (00-1 Campaign) - Le

SY-102 Grab Sample - (00-2 Compatibility) Blaak

TBD Grab Sample (00-2 Compatability) - Blaak

TBD Grab Sample (00-2 Carpaign) - Le

SY-102 Grab Sample - (01-i Compatibility) Blaak

TBD Grab Sample (01-1 Compatability) - Blaak

AZ-101 Grab Sample (NCE)

2000 rotal Grabs
$01-$ cet -97

$08-\infty$

$15-\infty \mathrm{ct}-97$

03-Dec-97

10-Dec -97

17-DeC-97

05-Jan-98

$74-J \operatorname{an}-98$

21-Jan-98

28-Jan-98

$04-F e b-98$

$13-\mathrm{Feb}-98$

20-Feb-98

02-Mar-98

$09-$ Mar-98

16-Mar-98

$27-\mathrm{Mar}-98$

07-Apr-98

22-Jun-98

29-Jun-98

20-Ju1-98

04-Aug-98

07-Auq-98

14-Rug-98

15-Aug-98

22-Aug 98

20-Aug-98

29-Aug-98

$02-500-98$

08-Sep-98

$24-\operatorname{Sep}-98$

29-Sep-98

$01-0<t-98$

$06-\infty \mathrm{ct}-98$

$11-\operatorname{\alpha ct}-98$

$20-\alpha c t-98$

27-0ct-98

01-Nov-98

Q2-Nov-98

08-Nov -98

$13-\mathrm{Nov}-98$

18-Nov-98

05-Dec-98

05-Jan-99

$01-$ Teb-99

01-Feb-99

15-Feb-99

01-Apr-99

20-Ju1-99

01-Sep-99

16-Sep-99

01-Nov-99

01-Nov-99

04-Jan-00

0 - $\mathrm{eeb}-00$

15 - Feb-00

$03-\not p r=00$

$01-\operatorname{Sep}-00$

18-Sep-00

21-NOV-0O $03-0<t-97$

10-0<t-97

$17-00 t-97$

05-Dec-97

12-Dec-97

19-Dec-9?

07-Jan-98

16-Jan-98

23-Jan-98

30-Jan-98

10-Eeb-98

17-Feb-98

25-Eeb-98

$04-\mathrm{Mar}-98$

11-Mar-98

20-Mar-98

02-Apr-98

07-Apr -98

24-Jun-98

$0:-J u 1-98$

22-Ju1-98

06-Rug-98

13-Aug-98

14-Rug-98

15-Aug-98

22-Aug-98

24-Aug-98

29-Aug-98

02-Sep-98

08-Sep-98

24-Sep-98

29-Sep-98

05-oct-98

$06-0$ ct -98

$11-0$ ct -98

22-Oct-98

27-0ct-98

01-Nov-98

04-Nov-98

$08-\mathrm{Nov}-98$

$33-\mathrm{Nov}-98$

18-Nov-98

05-Dec-98

03-Feb-99

$03-F \circ b-99$

17-Feb-99

$05-4 p r-99$

22-

03-Sep-99

$20-5 \mathrm{ep}-99$

03-Nov-99

03-Nov-99

06-Jan -00

03-Eeb-00

17- $\mathrm{Feb}-00$

$05-A p x=00$

06-Sep-00

20-Sep-00

23-Nov-00

AP $\quad 104$

$\begin{array}{ll}\mathrm{AP} & 108 \\ \mathrm{C} & 201\end{array}$

C 202

TX 302

U 301

AW 102

AY 102

S 302

AN 102

$\mathrm{AX} \quad 102$

SY 102

AP 103

SX 115

AW 101

AN 107

AN 102

AP 106

AP $\quad 104$

$\mathrm{Az} \quad 101$

A2 103

AZ $\quad 101$

AY 102

AY 102

AY 102

AP 107

AY 102

AY 102

AY 102

AY 102

AY 102

SY 102

AY 102

AY 102

AY TED

AY 102

z 361

AY 102

AY $\quad 102$

AY 102 
Table 6 (cont'd):

TWRS Characterization Grab Sample Projections 1998 - 2008

GSEY -

GSE2-4

GSAZ-4

GSCK-4

GSBD-4

GSCM-4
AZ-101 Grab Saraple Vendor cualify (NCE)

AN-105 Grab Sample (NCF)

AP-102 Grab Sample Process Control (5AN) (NCF)

AP-104 Grab Sample Process Control (5AN) (NCF)

AP-102 Grab Sample Feed Qualification (5AN) (NCF

AD-104 Grab Sample Eeed Qualification (5AM) (NCF

2001 Total Grabs

$04-J u n-01$

$07-\mathrm{Ju1}-01$

22-Aug-01

30-Aug -01

19-sep-01

27-Seo-01
08-Jun-01

09-Ju1-01

24-Aug- 01

$01-\mathrm{Sep}-01$

$25-$ Sep-ol

$03-0 c t-01$

$\begin{array}{ll}A Z & 101 \\ A N & 105 \\ A P & 102 \\ A P & 104 \\ A P & 102 \\ A P & 104\end{array}$

$02-J a n-02$

$16-\mathrm{Jan}-02$

$03-\mathrm{Mar}-02$

11-Mar-02

02-Apr-02

09-Apr -02

12-May 02

18-Ju2-02

21-Seo-02

$22-\mathrm{NOV}=02$
AZ-102 Grab Samp

GSFR-4

A2-102 Grab Sample (NCF)

2002 Total Grabs
23-Mar-03

$02-\mathrm{May}-03$

12-May-03

03-Jun-03

25-Jun-03

$04-A u g-03$

10-Oct-03

$23-\infty \mathrm{c} t-03$

$02-\mathrm{Dec}-03$

12-Dec-03

$15-\mathrm{Dec}-03$

30-Dec-03

AY-102 Grab Sample (NCF)

AP-102 Grab Sample Eeed Qualification (3AN) (NCF

2003 Total Grabs
$04-\mathrm{Jan}-02$

18-Jan-02

$05-\mathrm{Mar}-02$

$13-\mathrm{Mar}-02$

08-Aor-02

$15-\mathrm{Apr}-02$

14-May-02

20-Jul-02

$23-\mathrm{Sep}-02$

$02-\mathrm{Dec}-02$

$\begin{array}{ll}A Y & 101 \\ A N & 204 \\ A P & 102 \\ A P & 104 \\ A P & 102 \\ A P & 104 \\ A Z & 102 \\ A Z & 102 \\ A Z & 102 \\ A Z & 102\end{array}$

$25-\mathrm{Ma}-03$

$04-\mathrm{May}-03$

14-May-03

$09-J u n-03$

01-3ul-03

06-Aug-03

$12-$ oct -03

25-0ct-o3

04-Dec-03

14-Dec-03

19-Dec-03

05-Jan-04

$\begin{array}{ll}A W & 101 \\ A P & 102 \\ A P & 104 \\ A P & 102 \\ A P & 104 \\ A Y & 102 \\ A Y & 102 \\ A N & 103 \\ A P & 102 \\ A P & 104 \\ A Y & 102 \\ A P & 102\end{array}$

20-Jan-04

24-Eeb-04

19-Jun-04

19-Jun-04

11-Rug-04

16-Aug-04

$\begin{array}{ll}\text { AP } & 104 \\ \text { AY } & 102 \\ \text { AP } & 102 \\ \text { AP } & 104 \\ \text { A. } & 102 \\ \text { AP } & 104\end{array}$

$11-A$ pr -05

11-Apr-05

11-May-05

23-May-05

27-May-05

$18-J u n-05$

03-Sep-05

$03-\operatorname{cct}-05$

$03-0 c t-05$

08-Nov-05

$\begin{array}{ll}A P & 104 \\ A P & 102 \\ A N & 107 \\ A P & 102 \\ A P & 104 \\ A N & 107 \\ C & 104 \\ S X & 102 \\ A W & 105 \\ C & 104\end{array}$

12-Jan-06

14-Mar-06

$15-M a r-06$

17-Apr-06

39-May-06

23-Jun-06

26-Jul-06

11-Sep-06

17-0ct-06

14-Nov-06

27-Dec-06

$\begin{array}{ll}\text { C } & 104 \\ \text { SY } & 101 \\ \text { C } & 104 \\ \text { AP } & 102 \\ \text { AP } & 102 \\ \text { AP } & 104 \\ \text { A. } & 104 \\ \text { AP } & 102 \\ \text { AP } & 102 \\ \text { AP } & 104 \\ \text { AP } & 104\end{array}$


Table 6 (cont'd):

MNF-1641 REV. 0

TWRS Characterization Grab Sample Projections 1998 - 2008

GSBG-4

GSCE-4

$\mathrm{GSCD}-4$

GSTU-4

GSDN-4

GSCG-4

GSBZ-4

GSDX -4

GSDT -4
SY-103 Grab Sample Privatization (NCE)

AP-102 Grab Sample Process Control (1SY) (NCF)

AP-102 Grab Sample Feed Qualification (1SY) (NC

AP-104 Grab Sample Process Control (1SY) (NCF)

AP-104 Grab Sarple Feed Qualification (1SY) (NCF

AP-102 Grab Sample Process Control (3SX) (NCF)

2007 Total Grabs

AP-102 Grab Sample Eeed Qualification (3SY) (NCF AP-104 Grab Sample Process Control (3SX) (NCE)

AP-104 Grab Sample Feed Qualification (3SY) (NCF

2008 Total Grabs
09-Mar-07

$06-\mathrm{May}-07$

12-3un- -07

$11-$ Aug -07

$14-\mathrm{Aug}^{-07}$

30-Nov -07

$07-\operatorname{Jan}-08$ 02-Feb-08 11-Mar-08

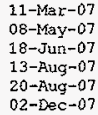

02-Dec-07

$11-\mathrm{Jan}-08$ $04-$ Eeb-08 17-Mar-os
SY 103

AP $\quad 102$

AP 102

AP 104

AP 104

AP 102

AP $\quad 102$

AP 204

AP $\quad 304$
5
5
5
5
5
5
30

5
5
5
15 
Table 7:

MNF-1641 REV. 0

TWRS Characterization Push Sample Projections 1998-2001

ACTIVITY

PS28-4

DS53-4

PS04-4

PS10-4

PS51- 4

P552-4

PS35-4

PS29-4

PS23-4

PS43-4

घS36-4

PS37-4

P545-4

PS46-4
TITLE

sX-106 push Samples 1 segments: 20-0ct-97 sX-106 push Sample 1 segments $1:$ AX-101 push samples 2 segments: S-102 push samples 1 Seg 11

S-111 push Sample $1 \mathrm{seg} 11$

U-109 Push Sample 1 seg 10

A-103 Push Samples 2 Segments 8

1998 Total Eush

13-Nov-97

$03-$ Dec -97

28-Jan-98

19-Mar-98

23-Aor -98

$08-0$ ct -98

S-103 Push Samples 2 Segments 6 SY-103 Push Samples 2 Segments:

AY-102 Push Sample 2 segruents 1

1999 Total push

$07-\mathrm{DeC}-98$ $01-\mathrm{Mar}-99$

08-oct-99

S-I12 Push Samples 2 segments 1 :

U-111 Rush Samples 2 segments 7

T-109 Push sample 2 segments 2

$07-D e c-99$

11-Feb-00

2000 Total Eash

23-0ct-00

20-Dec-00

T-103 push Sample 2 segments 1

2001 Total Fush
FINISH

FARA TANK

\#RISERS

\#sBgamearts
$1 \quad 11$

31-0ct-97 sx 106

\$X 106

AX 101

$\$ 102$

S 111

U 109

A 103

13-Jan-99 26-Mar-99 04-Nov-99

$\begin{array}{ll}\text { S } & \mathbf{1 0 3} \\ \text { SY } & 103 \\ \text { AY } & 102\end{array}$

2

2
2

33-Jan-00

10-Mar-00

17-Nov-00

$\begin{array}{ll}\mathrm{S} & 112 \\ \mathrm{U} & 111 \\ \mathrm{~T} & 109\end{array}$

2

2

24-Jan-01 T 103

2

1 
Table 8:

TWRS Characterization Rotary Sample Projections 1998 - 2003

ACTIVITY

TFTIE

RS96-4

RS45-4

RSAY - 4

RS67-4

RS42-4

RS44-4

RS2A-4

RS91-

RSAK -4

RS36-4

RS41-4

RS29-4

RS11-

RS38-

RS95-4

RS75-4

RSAV -4

RS) 3-4

RS33-4

RS71-4

FS32 - 4

RS12-4

RS16-4

RSAJ-4

RS78-4

RS53-4

RS77-4

RS87-

RS68-4

RS13-4

RS66-4

RS10-4

RS94-4

RS76-4

RS14-4

RS15-4

RS84-4

RS83-

RS46-4

RS17-4

RSAE-4

RS69-4

RSQ2-4

RS74-4

RS79-4

RSAH-4

RS21-4

RSO9-4

RS55-

RS72-

RS70-4

RS85-4

RSAE-4

RSAG-4

RS60-4

RS97-4

RS63-4

RS98-4

RS22-4

$R S 92-4$

1998 Total Rotary

1999 Total Rotary

2000 rotal Rotary 2001 Total Rotary 2002 Total Rotary

START

EINISE

FAFM TALTK

trisers "

C-202 Rotary Sample I Segments 1 -101 Rotary Sample 1 seg 8 High Eriority SX-101 Rotary Sample 1 Seg 9 High Rriority TX-104 Rotary Samples 2 Segments 2 SK-105 Rotary Samples 2 Segments 13

SX-103 Rotary Samples 2 Seg $13 \mathrm{High}$ Priority

TX-128 Rotary Samples 2 Segment 6 High Reiority s-110 Rotary Samole 2 Segments 8 High Rrioxity AZ-102 Rotary Sample 2 Segments 17

U-107 Rotary Samples 2 Segs 9 High Priority

BY-105 Rotary Samples 2 Segments 9 High Priority

BY-103 Rotary Samples 2 segments 9 High Priority

SX-104 Rotary Samples 2 Segment 12 High Priority

$S Y-101$ Rotary Sample 1 segment 22

C-104 Rotary Sample 2 Segment 5

TX-113 Rotary Samples 2 Segments it

2-361 Rotary Sample 2 Segments 6 Bogen (NCE)

Tx-111 Rotary Samoles 2 Segments 8 High Priority

sX-102 Rotary Samples 2 segments 11

TX-110 Rotary Samples 2 Segments 10

TX-105 Rotary Samples 2 segments 12

sX-109 Rotary Sample 2 Segment 5

SX-114 Rotary Samples 2 Segments 4

U-102 Rotary Sample 2 Segfnents 8 (6-8 only)

TX-116 Rotary Samples 2 Segments 12

A-106 Rotary Sample 2 Segments 2

TX-115 Rotary Samples 2 Segments 11

C-102 Rotary Samples 2 Segments 8

TX-306 Rotary samples 2 Segments 8

sX-210 Rotary Samoles 2 Segments

TX-102 Rotary Samples 2 secments 3

SX-107 Rotery Samples 2 Segments 2 rY-105 Rotary Samoles 2 segments TX-114 Rotary Samples 2 Segments 11 SX-111 Rotary Samples 2 Segments 3 SX-112 Rotary Samoles 2 Segments 2 TX-103 Rotary Samoles 2 segments $\mathrm{TX}-101$ Rotary Samples $Z$ Segments 3 SX-108 Rotary Samples 2 Segments 2

T-101 Rotary Samples 2 Segments 3 BY-101 Rotary Sample 2 Segment 8 TX-108 Rotary Samples 2 Segments 3 C-206 Rotary Samoles 2 segments 5 TX-112 Rotary Samples 2 Segments 13 $\mathrm{TX}-127$ Rotary Samples 2 Segments 10 BX-111 Rotary Sambles 2 Segments 4 TY-103 Rotary Samoles 2 Segments 4 BX-102 Rotary Sample 2 segment 2 8-105 Rotary samples 2 Segments 3 TY-102 Rotary Samoles 2 Segments 2

TX-109 Rotary Samples 2 Segments 8 U-104 Rotary Samples 2 Segments 2 C-204 Rotary Sample 2 Sample 1

C-101 Rotary Sample 2 segments 2 s-105 Rotary Samples 2 Segments? A-104 Rotary Samole 2 segments 1 S-108 Rotary Samples 2 Segments 9 A-105 Rotary Sample 2 Segments 1 TY-101 Rotary Samples 2 Segments 3 U-101 Rotary Sample 2 segments 1 2003 rotal Rotary 25-0ct-97

$01-D e c-97$

$06-\operatorname{Jan}-98$

09-Eeb-98

22-Eeb-98

$27-$ Feb-98

23-Mar-98

07-Mล $y-98$

13-May-98

16-Jun-98

$17-5 u 1-98$

$28+$ Rug-98

16-Sep-98

$01-$ Dec-98

20-Nov-98

21-Jan-99

01-Eeb-99

02-Feb- 99

13-Ap F-99

03-May -99

06-Ju1-99

01-sep-9

26-0ct-99

19-Nov-99

$21-\operatorname{oct}-97$ 16-Dec-97 $21-\mathrm{Jan}-98$ 13-Ma $\mathrm{M}-98$ 18-Mar-98

01-Apr-98 23-Apr $=98$

10-Jun-98

16-Jun-98

21-JuL -98

19-Aug-98

01-0ct-98

19-0ct-98

16-Dec-98

24-Eeb-99

05-Mar-99

08-Ma $c-99$

14-May-99

$04-J u n-99$

06-Aug-99

$05-0<t-99$

$30-N o v-99$

27-Jan-0

08-Mar-00

23-Mar-00

23-May-00

20-Jul-00

20-Sep-00

21-sep-00

OS-Jan-00

01-Mar-00

10-Apr $=00$

25-Apr-00

26-Jun-00

22-Aug-00

$23-0 c t-00$

24-0ct-00

$07-$ Dec-00

12- Eeb-01

30-ApL-01

03-May-01

20-Ju1-01

21-Aug-01

16-Oct-01

$22-0 c t-0$

17-Jan-01

16-Mar-01

01-งun-01

06-Jun-01

22-Aug-01

24-5ep-01

16-Nov-01

26-Nov-01

12-Dec-01

17-Jan-0?

25-Eeb-02

21-Mar-02

12-Apr-02

30-May-02

08-5ul-02

29-Jul-02

og-sep-02

11-0ct-02

14 - 0 ct -02

23-Jan-02

20-Eeb-02

28-Mar-02

23-Apr-02

$15-4 a y-02$

03-Jul-02

08-Aug-02

29-Aug-02

10-0ct-02

$13-\mathrm{Nov}-02$

24-NOV-O2

14-3an-03

04-Mar-03

04-Mar-03

21-Apr-03

14-MaY-03

16-Ju1-03

22-Aug $=03$

17-0ct-03

24-0ct-03

01-Dec-03

$16-\mathrm{sep}-03$

23-Sep-03

27-oct-03

$\begin{array}{ll}C & 202 \\ \text { SX } & 101 \\ \text { SX } & 101 \\ \mathrm{TX} & 104 \\ \mathrm{SX} & 105 \\ \mathrm{SX} & 103 \\ \mathrm{TX} & 128 \\ \mathrm{~S} & 110 \\ \mathrm{AZ} & 102 \\ \mathrm{U} & 107 \\ \text { BY } & 105 \\ \text { BY } & 103 \\ \text { SX } & 104 \\ \text { SY } & 101\end{array}$

$\begin{array}{rr}1 & 1 \\ 1 & 9 \\ 1 & 9 \\ 2 & 2 \\ 2 & 13 \\ 2 & 13 \\ 2 & 6 \\ 2 & 8 \\ 2 & 17 \\ 2 & 9 \\ 2 & 9 \\ 2 & 9 \\ 2 & 12 \\ 1 & 22\end{array}$

275

$\begin{array}{ll}\mathrm{CX} & 104 \\ \mathrm{TX} & 113 \\ \mathrm{TX} & 361 \\ \mathrm{SX} & 111 \\ \mathrm{TX} & 102 \\ \mathrm{TX} & 110 \\ \mathrm{SX} & 105 \\ \mathrm{SX} & 114\end{array}$

$\begin{array}{ll}\mathrm{U} & 102 \\ \mathrm{TX} & 116 \\ \mathrm{~A} & 106 \\ \mathrm{TX} & 115 \\ \mathrm{C} & 102 \\ \mathrm{TX} & 106 \\ \mathrm{SX} & 110 \\ \mathrm{TX} & 102\end{array}$

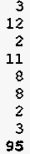

$\begin{array}{llrr}\text { SX } & 107 & 2 & 2 \\ \text { TY } & 105 & 2 & 5 \\ \text { TX } & 114 & 2 & 11 \\ \text { SX } & 111 & 2 & 3 \\ \text { SX } & 112 & 2 & 2 \\ \text { TX } & 103 & 2 & 3 \\ \text { TX } & 101 & 2 & 3 \\ \text { SX } & 108 & 2 & 2\end{array}$

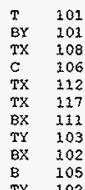

2
2
2
2
2
2
2
2

TX 109

U 104

C 204

$\begin{array}{ll}\text { C } & 101 \\ \text { S } & 105\end{array}$

A 104

108

A 105

$\begin{array}{ll}\text { TY } & 101 \\ \mathrm{~V} & 101\end{array}$
62

2
5
1
3
2
3
3
2
2

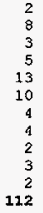

112

8
2
1
2
9
1
9
1
3
1
74


Figure 5:

HNF-1641 REV. 0

Comparison of the Cumulative Archive Jar Inventory Under Various Scenarios
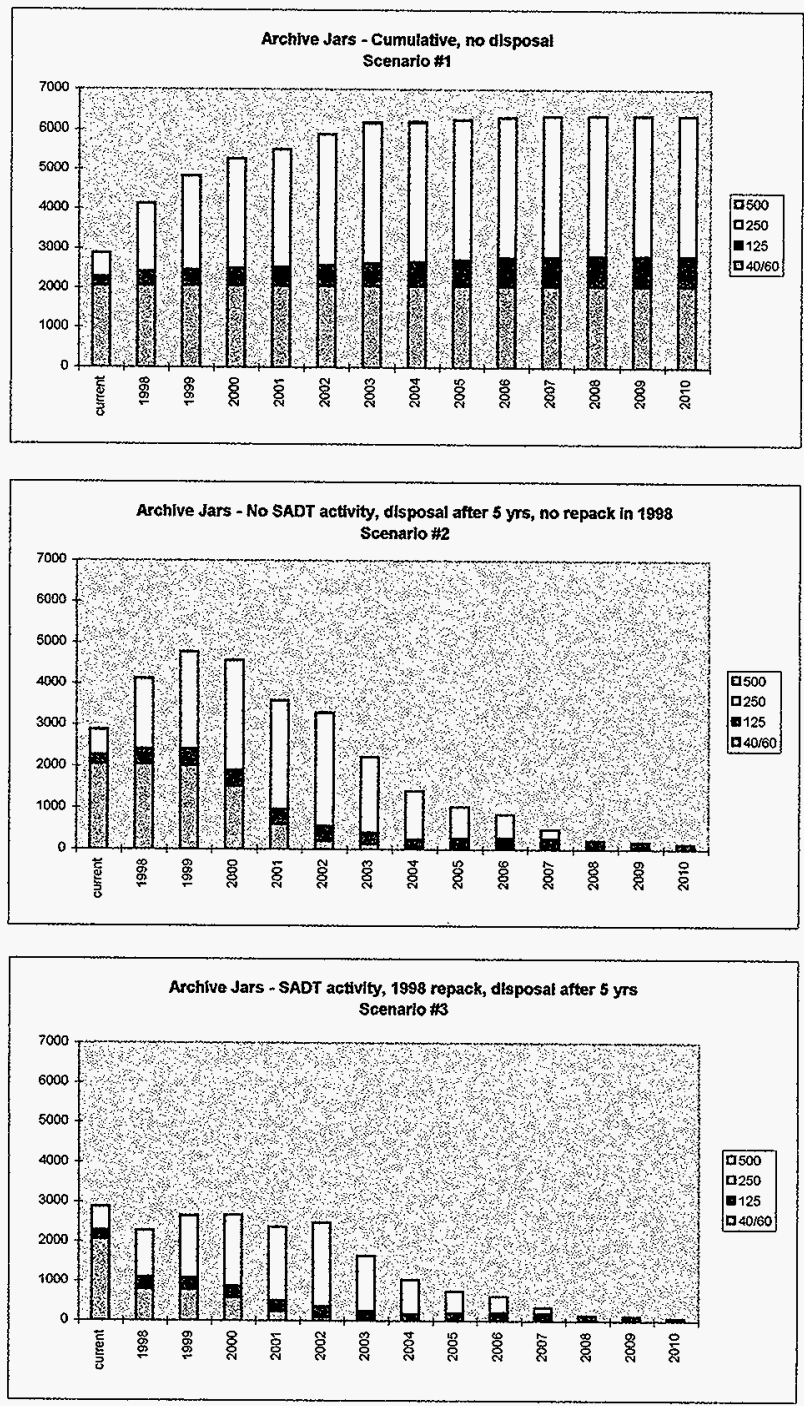


\section{HNF-1641 REV. 0}

- combination of archive and parent jars into only one jar.

Appendix II includes tables from the current inventory database which quantify the sample jars potentially affected by each of the repackaging activities

The SADT assumption includes:

- elimination of approximately $50 \%$ of the jars due to SADT activities/recommendations in 1988

- elimination of $25 \%$ of each previous year's samples from 1999 on.

Figure 6 shows the potential effect of repackaging and 1998 SADT activities. This scenario results in the lowest jar projections of the three, with a maximum of 3450 jars in 2000.

All scenarios show a gradual transition from small jars ( 40 and $60 \mathrm{~mL}$ ) to larger jars $(125 \mathrm{~mL}$ and $250 \mathrm{~mL})$ over time. This is due to the general increase in sample recoveries over the past years. It is also the result of not splitting the samples into parent and archive jars at extrusion.

The majority of the 222-S laboratory sample storage trays and bins are designed for optimum packing using $60 \mathrm{~mL}$ vials. Until recently all TWRS guidance was to save a $20-40 \mathrm{~mL}$ vial of material. The laboratory has increased the volume of the archive sample to $60 \mathrm{~mL}$ because the material is easier to retrieve, homogenize and subsample in the larger vials. It is now acknowledged that, even if more than 75 grams is recovered, it is all expected to be retained. This will inevitabley result in increasing numbers of 125 and $250 \mathrm{~mL}$ jars and decreasing numbers of $60 \mathrm{~mL}$ jars.

The 222-S laboratory must engineer new trays and containers which are more compatible with the larger jars which will comprise the inventory in coming years. Currently, the "baskets" represent the most efficient way to store the larger jars. To implement scenario 3 , using only these baskets, would require 213 baskets; scenario 2 would require 136 baskets; scenario 1 would require 121 baskets. 222-S currently has only 32 baskets. However, in scenario 2 and three, the maximum storage requirements come in the year 2002 , some years away. in addition, the wall shelf space now devoted to small $40 / 60 \mathrm{~mL}$ jars would become available at the same time, reducing the need for baskets.

If the laboratory manages the inventory more like the scenario 3 and less like scenario 1 , then the $11 \mathrm{~A}$ cells should provide enough inventory space. If additional space is necessary, the $1 \mathrm{~F}$ hotcell could provide additional space. However, if used, $1 \mathrm{~F}$ would require samples to be loaded out of $11 \mathrm{~A}$. This 


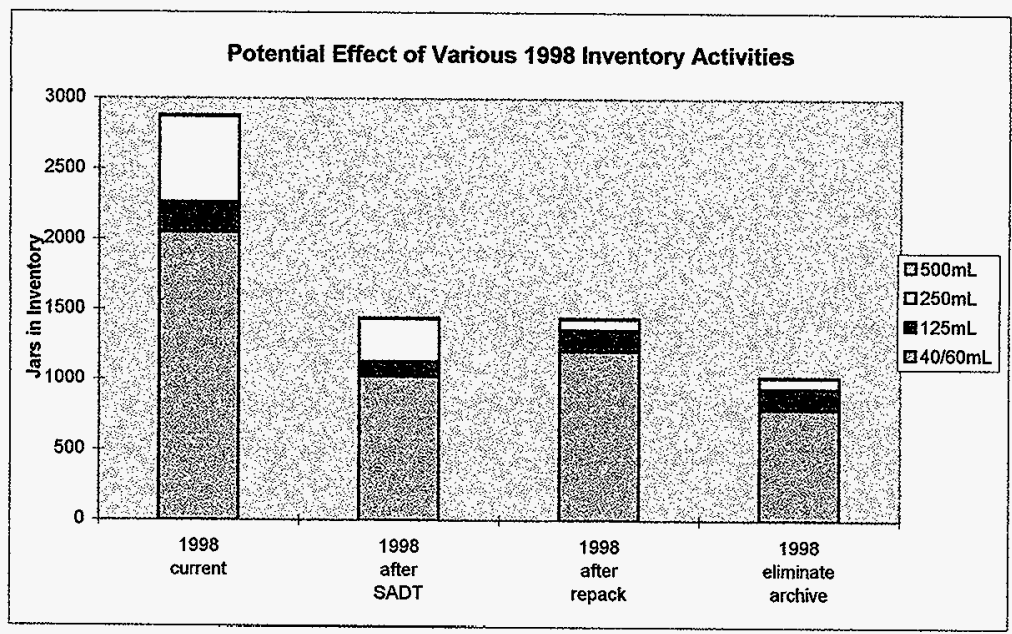

\begin{tabular}{|l|r|r|r|r|r|}
\hline & $40760 \mathrm{~mL}$ & $125 \mathrm{~mL}$ & $250 \mathrm{~mL}$ & $500 \mathrm{~mL}$ & TOTAL \\
\hline 1998 current & 2050 & 209 & 612 & 11 & 2882 \\
\hline 1998 after SADT & 1025 & 105 & 306 & 6 & 1442 \\
\hline 1998 after repack & 1210 & 146 & 80 & 6 & 1442 \\
\hline 1998 eliminate archiv & 793 & 146 & 80 & 6 & 1025 \\
\hline
\end{tabular}




\section{MNF-1641 REY O}

activity would provide radiological dose to 222-S laboratory employees and some risk of facility contamination during the transfer.

Additionally, $1 F$, if used at all for sample storage, should only receive older samples, after their 2-3-year repackaging. This would ensure that $1 \mathrm{~F}$ samples represent those samples least likely to require any significant operational attention until a decision is made to disposition.

The 222-S laboratory hotcell locations are posted and maintained as HRA (highlevel radioactivity areas) and are ventilation-controlled. Therefore, there would be no maintenance or surveillance in addition to that already in place for the management of the hotcells themselves and the activities described above. In addition, load-out procedures for transfer of materials between the $11 \mathrm{~A}$ and $1 \mathrm{~F}$ hotcells exist and could be implemented if necessary. 


\section{Maintenance of Archived Samples}

While in the custody of the 222-S laboratory, the tank waste material must be packaged and stored under conditions which will minimize the deterioration of the samples. In addition to the specific packaging, some maintenance of the archive samples is required to ensure that samples are retrievable and that the laboratory is continuously aware of the number of sample containers and amount of waste in each container.

The results of the physical inventory conducted in March of 1998 shows that the lack of a physical inventory for four years did not affect the sample mass estimates critically. More than $80 \%$ of the samples were actually measured to be within $+1-20 \%$ of their estimated mass. The process revealed 40 samples ( $1 \%$ of the total) which existed but were not in the database, and 120 samples ( $4 \%$ of the total) for which the database indicated some remaining material, but which had actually been depleted. Approximately 20 jars $(0.7 \%)$ were broken during the inventory process and had to be repackaged. Physical inventories, involving approximately 2 man-months of effort, are absolutely necessary to reconcile actual inventory with the database periodically, but perhaps not on an annual basis. A biennial physical inventory would represent a reasonable balance between the minimization of physical handling of the samples and reconciliation of the database.

More frequent visual surveillance of the sample storage areas would have to be initiated to check for package deterioration and leakage. This surveillance should be done on a time-scale which would enable prompt repackaging of broken or cracked jars to limit sample loss or degradation. A balance must be struck between unnecessary physical handling of the samples and immediate identification of a problem. Daily inspection of the storage area for leaks and monthly visual inspection of the storage bins for sample package deterioration provides a reasonable balance.

There is a considerable backlog of sample jars which are older than 2 years and the first SADT query is yet to be completed. This will undoubtedly result in a significant repackaging effort in the short-term. Table 9 and figure 7 show the projected repackaging costs from 1998 through 2010. These projections assume the following:

- the SADT will request retention of $60 \%$ of the samples currently inhouse

- additional samples are generated per TWRS Sample Schedule Rev 5.3 (3/11/1998)

- samples will be repackaged during the year after they turn 3 years old

- repackaging costs are \$135/jar (per Narquis, 1997) 


\section{MNF-1641 REV. 0}

- samples will only be repackaged once

- all costs are in 1998 dollars (no inflation/discount rates applied)

Table 9 and figure 7 also show that the repackaging costs vary wildly during the first four years. Active management could smooth these expenditures by extending some of the jar lives for an additional 6 months, and repackaging others somewhat sooner than three years. One smoothed cost projection is shown table 9 and figure 7 . This approach simply averaged year $1 \& 2,3 \& 4,5 \& 6$, etc. 
Table 9:

HNF-1641 REV. 0

Cost Projections for Repackaging Activities 1998-2010

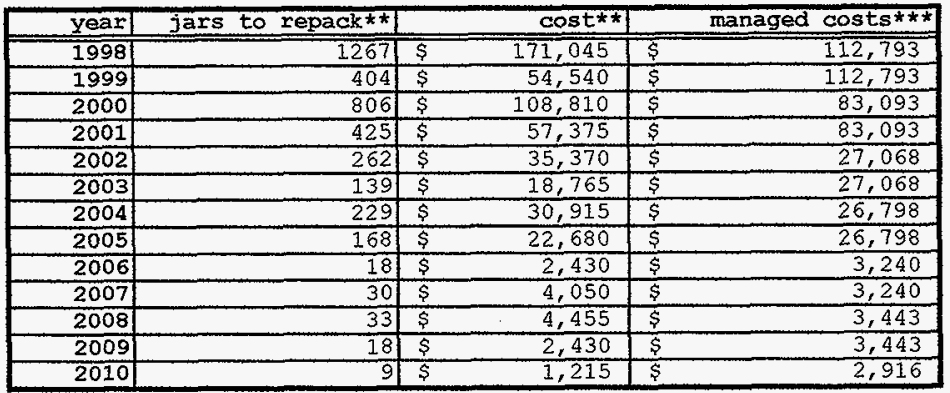

*assumes 608 retention by SADT

**assumes $\$ 135 /$ jar

$\star * *$ average 1998,1999 costs; 2000, 2001costs; etc. 
Figure 7:

HNF-1641 REV. 0

\section{Cost Projections for Repackaging Activities 1998-2010}

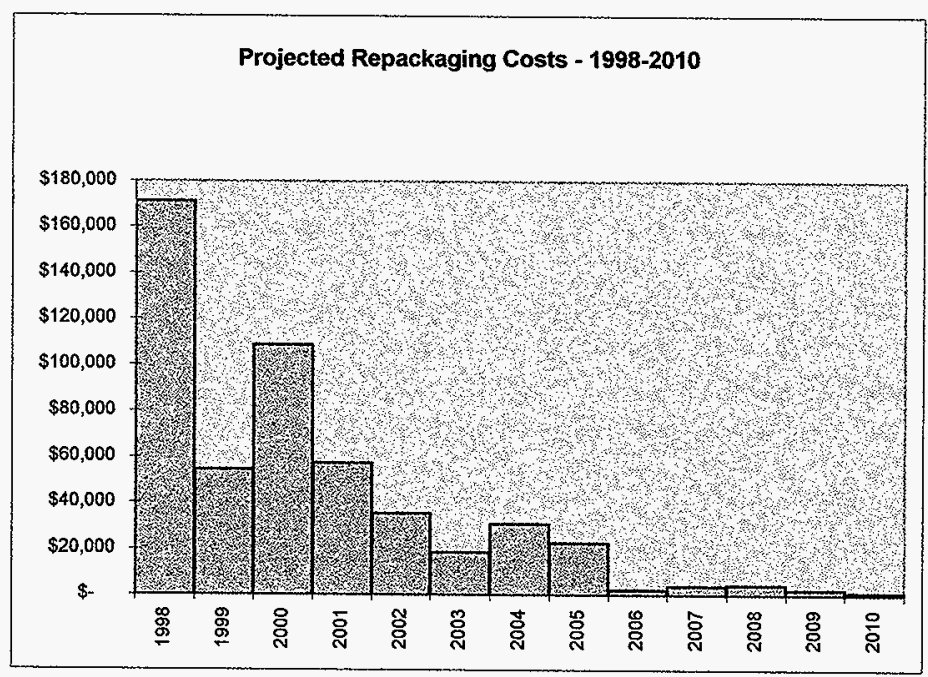

Repackaging costs - one way to manage

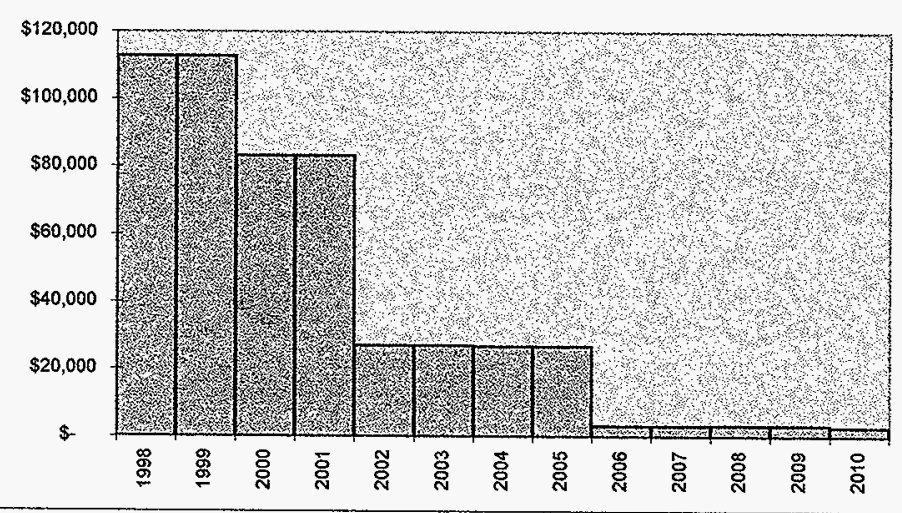




\section{Consolidation of Existing Databases}

Sample jars are managed at the 222-S laboratory through the use of two databases, both using the Borland PARADOX software. The main database used by the hotcell operators contains details of all of the samples generated through the extrusion and subsampling activities conducted in the 11-A hotcells.

The other database is maintained by the 222-S laboratory sample custodians and contains details of samples which are received into the laboratory directly without having to be extruded in $11 \mathrm{~A}$. This database also covers samples which are stored in $2 \mathrm{~B}$ and $2 \mathrm{E}$ storage areas.

There are very few TWRS Characterization archive samples which are stored in $2 \mathrm{~B}$ and, therefore, are not included in the hotcell database. These tend to be grab samples in $125 \mathrm{~mL}$ jars. Nevertheless, it would be an improvement to combine the databases and enable a single query to access all archive samples.

Another benefit of a single database would be to enable the sample inventory databases to continuously and accurately accomplish the radioisotope inventory calculations needed to satisfy the laboratory's only safety operating limit. The 222-S laboratory is obliged to maintain records which record the radioisotopic inventory in the facility at any point in time. This is the single operating limit in the facility safety basis ( $r$ f for ISB). The inventory accounts for tank waste samples in a conservative way by assuming that each core segment is fully recovered. This may be modified by reducing the inventory by the appropriate factor once the extrusion is performed and the percent recovery is known. There is currently no mechanism to account for material which is used-up during the chemical analysis process or material which is dispositioned down the 219-S liquid waste system.

Since the facility isotopic inventory represents a safety operating limit, the associated databases must be $Q A$ validated and maintained in accordance with site and facility software $Q A$ requirements. The current inventory/archive database is not so validated and maintained.

Activities are underway to accomplish this transition. The new database will be based on Microsoft ACCESS, and will be more formally maintained, fully consistent with site software $Q A$ and maintenance requirements. 


\section{Disposition}

Both the TWRS Characterization organization and the Analytical Services Organization have studied various disposition options over the past five years. These options range from dissolving/slurying the samples and pouring them into the 222-S laboratory liquid waste system to building a new building at the Central Waste Complex for retrievable long-term storage.

Three potential disposition scenarios were considered:

- Return of the material to a TWRS tank via the 219-S Waste Handling Facility and cross-site transfer line

- Liquids removal and packaging the waste for storage at the Central Waste Complex (CWC)

- Packaging the waste and transporting the material to TWRS to be returned to the parent tank.

Use of the 222-S laboratory liquid-waste handling system through the 219-S Waste Handling Facility represents the option which minimizes personnel dose and results in the least long-term concerns with the material. Waste repackaging involves loading-out materials from the hot cells, packaging, physical transportation, storage and management and still leaves ultimate disposal unresolved. Returning the material to the parent tank also involves unreasonable personnel exposures as well as costly planning and tank-access activities.

Finally, CWC is not currently permitted to store high-activity waste and significant additional cost would be incurred to establish this capability.

Effectively transferring the sample material to the 219-S Waste Handling Facility involves dumping liquid and slurry samples directly down the hotcell drains, while simultaneously flowing water. Solid samples would, ideally, be dissolved in water or weak acid. However, it is known from historical experience that some solids will remain. These must be effectively flushed down the 222-S laboratory drainlines using clean rinsewater.

Procedures are in place, and are being updated, to properly transfer the waste materials to the 219-S Waste Handling Facility. These procedures must address material hold-up in the drain lines, local low-spots in the drainlines, potential increase in 222-S laboratory tunnel general doserates in the vicinity of the hotcell drain lines, and adherence to the waste acceptance criteria for eventual transfer of the liquid waste to the tank farms via the cross-site transfer line. 


\section{MNF-1641 REV. 0}

Much of the solid material in the Tank samples is crystalline and solid simply due to supersaturation. These salts would be expected to remain in solution once introduced into the 219-S Waste Handling Facility. However, some solids would be expected to re-crystalize within the 219-S tanks when the $\mathrm{pH}$ is raised in preparation for transfer. The resulting solids must be accounted for prior to introduction of the material into the 219-S system. 


\section{Regulatory Considerations}

The TWRS samples are archived at the 222-S laboratory in accordance with the sample exclusion provision of the Washington Dangerous Waste Regulations (WAC 173-303-071(3)(l)). This states that as long as the sample material is retained for a specific purpose, the samples are not managed as a dangerous waste. This exclusion is not indefinite and extends only as long as the integrity of the sample remains sufficient to perform legitimate characterization testing.

A second exclusion, WAC 173-303-071(3)(s), applies only to treated material from treatability studies. This treated material may be archived for a period of 5 years from receipt of the original treatability sample at the laboratory/testing facility.

\section{Legitimate retention}

One reason for returning to a sample for characterization is to recheck previously-performed chemical analyses. Given environmental regulatory holding times and the inevitable moisture loss during storage, the integrity of the sample for sample recheck is limited to a year or so for many constituents. Some, including total metals and radionuclide elemental analysis, could be performed years after the original testing.

Another reason for retaining a sample for characterization is to enable some limited testing on retained samples to answer new questions. It is conceivable that some chemical analysis, or physical testing, even on compromised samples, would provide useful information. The window of usefulness for this purpose may extend past a year or two.

A third reason for sample retention is to characterize the material by performing some bench processing experiments (treatability studies). These could be compatibility of two or more waste streams, interaction with water or salt solutions, or pretreatment-treatment simulations. These characterization would require relatively larger samples. In addition, the viability of the samples may extend to five years or even greater, depending upon the nature of the experiments.

A key concept is that a documented, legitimate use is necessary to retain the sample exclusion for the archive material. Within the program, samples will be retained as is, or may be combined into various composites. Individual samples will be retained for a year as a matter of course, to enable rechecking of laboratory tests and to allow time to canvass the Hanford programs for interest in retention. The SADT mechanism described in HNF-SD-WM-TRD-006 will be used to identify interest in specific-sample retention. 
The laboratory will, in conjunction with TWRS Characterization SADT and the programs which wish to retain the sample, propose sample consolidation schemes which will increase sample size and decrease sample number. This serves to maximize the ability of the lab to maintain good sample integrity, and keep the sample deterioration to a minimum. However, this consolidation will only be accomplished to the extent that the resulting sample composites are useful to the requesting programs.

In addition to specific requests from the programs, 222-S laboratory will identify generic program requests. These would be expected mostly from the privatization programs which may continue to need tank waste material for the duration of their programs.

\section{Treatability Test Sample Status}

If the sample material is to be used for treatability testing, the documentation and management activities required by WAC 173-303-071 are applicable. This includes documentation of the identification and mass of sample, treatment details, and nature of residuals. These activities are in place at the 222-S laboratory.

\section{Regulatory Status of Sample Disposition from the 222-S laboratory}

In order to effectively transfer waste samples from the hotcell locations into the 219-S Waste Handling Facility, certain activities will be required. These activities include dissolution in water or dilute acid, which may be interpreted as "waste treatment." WMH and FDH believes that this activity is legitimate "treatment-bygenerator" (WAC 173-303-802). It also represents the disposition approach which is most protective of human health and the environment both during the disposition process and upon ultimate disposition.

The dissolution process will take place within the physically- and atmosphericallycontained hotcells. Procedures to be used for this disposition process exist (ref). There is currently a work team of Numatec and Waste Management scientists revising the procedures to ensure compliance with new waste acceptance criteria for the 219-S Waste Handling Facility. Within these procedures any dissolution is necessary only to effect the transfer of the relatively small amounts of waste from the sample jars in the hot cells to the 219-S Waste Handling Facility, through the gravity-feed drainlines. This approach can be accomplished totally remotely, and separates the waste itself from the rinsed jars, which are loadedout and packaged as high-level mixed solid waste. 


\section{HNF-1641 REV. 0}

\section{Implementation}

The following activities will be required to implement the sample archive/inventory program:

- Build a sample-by-sample management plan for existing inventory after receiving first SADT letter

- Install new storage trays and racks in the 11A2 cell

- Evaluate and purchase necessary Hastelloy or Stainless steel containers for long-term storage

- Negotiate and document a generic defensible programmatic need for generic retention of samples or composites after the SADT indication that there is no longer a specific need

- Write a plan for accomplishing the repackaging and/or disposition of existing samples

- Write repackaging procedures

- Revision disposition procedures as necessary

- Write implementing procedures for the new archive program

- Train affected personnel in the new system

- Develop a new archive database which can interface with the CES system which monitors laboratory isotope inventory to manage against the 222-S laboratory safety basis 


\section{HNF-1641 REV. 0}

References

Operating Procedure, LO-100-171, Rev F-1, Waste Disposal at Hood 16 in Room 2B, Weaver, L. L., March 5, 1998

Operating Procedure, LO-100-107, Rev C-0, Cubicle Housekeeping, Waste Disposal, and Management, Griffin, B., II, June 3, 1997

Operating Procedure, LO-100-151, Rev D-0, Segregate and Manage Solid Laboratory Wastes, Warwick, G. J., August 20, 1997

WHC-SD-WM-ER-334, 222-S Laboratory Storage, Engineering Report, McVey, C., June 10, 1994

Engineering Evaluation, Evaluation of Sample Disposal of Non-Archive Sample Material at 222-S, McVey, C, March 1996, transmitted by Internal Memo, S. L. Brey to G. B. Griffin, April 8, 1996

WHC-SD-W079-ES-001, Rev 3, Hot Sample Archiving, McVey, C. B., May 26, 1995

Archive \& Excess Sample Storage/Disposition Cost Estimate, Hall, V. W., Internal Memo 75720-96-010, V. W. Hall to T. J. Kelley, September 24, 1996

Archived 222-S 11A Hot Cell Samples, Reasons for Retention, Path for Disposal, Beck, M., Internal Memo 8C510-97-037, Mark Beck to D. S. Mantooth, December 17, 1977

Archiving Requirements, Valenzuela, B. D., Internal Memo 7E720-94-125 from D. R. Bratzel to S. M. Joyce, June 23, 1994

CBM-94-002, Engineering Report for 222-S Laboratory Storage, McVey, C. B., April 1994.

White Paper - Storage of Excess TWRS Characterization Samples, Narquis, C. T., Internal Memo C. T. Narquis to L. F. Perkins [Date and Memo \#)]

HNF-SD-WM-SD-023, Rev 0., Fiscal Year 1997 Memorandum of Understanding for the TWRS Characterization Project, Schreiber, Ruth D., February 5,1997 
Sample Disposal via 219-S Facility, Beck, Mark A., Internal Memo 8C51097-009, Mark A. Beck to K. S. Tollefson, May 14, 1997

WHC-SD-WM-TI-569, Rev 0, Tank Core Sample Archiving Container Fabrication Material, Strong, K. C., April 29, 1993

WAC 173-303, Dangerous Waste Regulations, Washington State Deparatment of Ecology, as amended.

HNF-SD-WM-TRD-006, Technical Basis for Disposition of Tank Waste Samples in Archive (Adams, M.R., et al), March 1997 
HNF-1641 REV. 0

APPENDIXI

222-S Archive Database sorted by Tank:Core:Matrix 
Vials in 11A1 and 11A2

\begin{tabular}{|c|c|c|c|c|c|c|c|c|}
\hline Tank \# & Core \# & Matrix & Segment \# & Date & Jar/Vial \# & Vial Size (mLs) & Net Wt\# Found (gms) & Laboratory \# \\
\hline & & . & & & $\begin{array}{r}11919 \\
7188\end{array}$ & $\begin{array}{c}250 \\
40 \\
\text { Total Weight }\end{array}$ & $\begin{array}{c}215 \\
11.8 \\
226.8\end{array}$ & \\
\hline & $\begin{array}{l}\text { Grab } \\
\text { Grab } \\
\text { Grab } \\
\text { Grab }\end{array}$ & $\begin{array}{l}\text { GL } \\
\text { GL } \\
\text { GL } \\
\text { GL }\end{array}$ & $\begin{array}{l}10 \\
11 \\
C-1 \\
\mathrm{H} 1\end{array}$ & & $\begin{array}{r}\text { PH-10 } \\
\text { PH-11 } \\
\text { PH-11C1 } \\
\text { ENV-H1 }\end{array}$ & $\begin{array}{c}125 \\
125 \\
125 \\
125 \\
\text { Total Weight }\end{array}$ & $\begin{array}{c}150.1 \\
124.7 \\
163.4 \\
29.3 \\
467.5\end{array}$ & \\
\hline Tank \# & Core \# & Matrix & Segment \# & Date & Jar/Vial \# & Vial Size (mLs) & Net Wt\# Found (gms) & Laboratory \# \\
\hline $219-S$ & Grab & GL & & $1 / 21 / 98$ & 13876 & $\begin{array}{c}40 \\
\text { Total Weight }\end{array}$ & $\frac{40.1}{40.1}$ & S98M000021 \\
\hline Tank \# & Core \# & Matrix & Segment \# & Date & Jar/Vial \# & Vial Size (mLs) & Net Wt\# Found (gms) & Laboratory \# \\
\hline 972-SLY & & $\mathrm{GL}$ & & $10 / 2 / 97$ & 13500 & $\begin{array}{c}60 \\
\text { Total Weight }\end{array}$ & $\frac{13.8}{13.8}$ & S97A000065 \\
\hline Tank\# & Core \# & Matrix & Segment \# & Date & Jar/Vial \# & Vial Size (mLs) & Net Wt\# Found (gms) & Laboratory \# \\
\hline $\begin{array}{l}\text { A-101 } \\
A-101 \\
A-101 \\
A-101 \\
A-101 \\
A-101 \\
A-101 \\
A-101 \\
A-101 \\
A-101 \\
A-101 \\
A-101\end{array}$ & $\begin{array}{l}154 \\
154 \\
154 \\
154 \\
154 \\
154 \\
154 \\
154 \\
154 \\
154 \\
154 \\
154\end{array}$ & $\begin{array}{l}\text { DL } \\
\text { DL } \\
D L \\
D L \\
D L \\
D L \\
D L \\
D L \\
D L \\
D L \\
D L \\
D L\end{array}$ & $\begin{array}{l}11 \\
13 \\
13 \\
14 \\
14 \\
15 \\
16 \\
17 \\
17 \\
18 \\
18 \\
19\end{array}$ & $\begin{array}{r}8 / 1 / 96 \\
7 / 24 / 96 \\
8 / 1 / 96 \\
7 / 24 / 96 \\
8 / 1 / 96 \\
8 / 2 / 96 \\
8 / 2 / 96 \\
8 / 21 / 96 \\
9 / 16 / 96 \\
8 / 26 / 96 \\
9 / 6 / 96 \\
9 / 6 / 96\end{array}$ & $\begin{array}{r}10756 \\
10571 \\
10760 \\
10567 \\
10759 \\
9936 \\
10858 \\
11071 \\
11253 \\
11168 \\
11254 \\
11256\end{array}$ & $\begin{array}{c}40 \\
250 \\
40 \\
250 \\
40 \\
40 \\
40 \\
250 \\
60 \\
250 \\
60 \\
60 \\
\text { Total Weight }\end{array}$ & $\begin{array}{c}32 \\
231.8 \\
50.5 \\
180.2 \\
58.1 \\
57.8 \\
53.3 \\
208.8 \\
48.6 \\
210.8 \\
91.1 \\
34.9 \\
1257.9\end{array}$ & $\begin{array}{l}\text { S96T004109 } \\
\text { S96T004060 } \\
\text { S96T004111 } \\
\text { S96T004061 } \\
\text { S96T004112 } \\
\text { S96T004113 } \\
\text { S96T004114 } \\
\text { S96T004520 } \\
\text { S96T004675 } \\
\text { S96T004655 } \\
\text { S96T004673 } \\
\text { S96T004676 }\end{array}$ \\
\hline
\end{tabular}


Vials in 11A1 and 11A2

\begin{tabular}{|c|c|c|c|c|c|c|c|c|}
\hline Tank \# & Core \# & Matrix & Segment \# & Date & Jar/Vial \# & Vial Size (mLs) & Net Wt\# Found (gms) & Laboratory \# \\
\hline $\begin{array}{l}A-101 \\
A-101\end{array}$ & $\begin{array}{l}154 \\
154\end{array}$ & $\begin{array}{l}\text { FB } \\
\text { FB }\end{array}$ & $\begin{array}{l}\text { Fblk } \\
\text { Fblk }\end{array}$ & $\begin{array}{l}8 / 26 / 96 \\
8 / 21 / 98\end{array}$ & $\begin{array}{l}11067 \\
11102\end{array}$ & $\begin{array}{c}250 \\
40 \\
\text { Total Weight }\end{array}$ & $\begin{array}{c}182.5 \\
36.9 \\
219.4\end{array}$ & $\begin{array}{l}\text { S96T004654 } \\
\text { S96T004674 }\end{array}$ \\
\hline$A-101$ & 154 & Salt & 1 & $9 / 3 / 96$ & 10966 & 60 & 8.6 & S96T004677 \\
\hline A-101 & 154 & Salt & 1 & $9 / 3 / 96$ & 11136 & 60 & 78.5 & S96T004678 \\
\hline A-101 & 154 & Salt & 10 & $7 / 31 / 96$ & 10837 & 60 & 84.5 & S96T004081 \\
\hline A-101 & 154 & Salt & 10 & $8 / 1 / 96$ & 10838 & 60 & 24.7 & S96T004080 \\
\hline A- 101 & 154 & Salt & 13 & $8 / 1 / 96$ & 10839 & 60 & 30.2 & S96T004082 \\
\hline A-101 & 154 & Salt & 14 & $7 / 24 / 96$ & 10681 & 60 & 30.4 & S96T004083 \\
\hline A-101 & 154 & Salt & 15 & $8 / 2 / 96$ & 10841 & 60 & 87.2 & S96T004084 \\
\hline A-101 & 154 & Salt & 16 & $8 / 2 / 96$ & 10842 & 60 & 80.2 & S96T004085 \\
\hline$A-101$ & 154 & Salt & 18 & $9 / 6 / 96$ & 11255 & 60 & 60.4 & S96T004704 \\
\hline A-101 & 154 & Salt & 19 & $9 / 6 / 96$ & 11257 & 60 & 28.1 & S96T004703 \\
\hline A-101 & 154 & Salt & 2 & $9 / 3 / 96$ & 11137 & 60 & 99.3 & S96T004679 \\
\hline A-101 & 154 & Salt & 2 & $9 / 4 / 96$ & 11138 & 60 & 100.9 & S96T004680 \\
\hline A-101 & 154 & Salt & 3 & $9 / 4 / 96$ & 11242 & 60 & 97.5 & S96T004682 \\
\hline A-101 & 154 & Salt & 3 & $9 / 4 / 96$ & 11243 & 60 & 99.1 & S96T004681 \\
\hline$A-101$ & 154 & Salt & 4 & $9 / 5 / 96$ & 11246 & 60 & 89.7 & S96T004684 \\
\hline A-101 & 154 & Salt & 4 & $9 / 5 / 96$ & 11247 & 60 & 104.3 & S96T004683 \\
\hline$A-101$ & 154 & Salt & 6 & $9 / 5 / 96$ & 11248 & 60 & 94.6 & S96T004686 \\
\hline A-101 & 154 & Salt & 6 & $9 / 5 / 96$ & 11249 & 60 & 30.6 & S96T004685 \\
\hline A-101 & 154 & Salt & 7 & $7 / 31 / 96$ & 10835 & 60 & 94.1 & S96T004079 \\
\hline A-101 & 154 & Salt & 7 & $7 / 31 / 96$ & 10836 & 60 & 91.7 & S96T004068 \\
\hline A-101 & 154 & Salt & 9 & $8 / 26 / 96$ & 11166 & 250 & 69.1 & S96T004668 \\
\hline A-101 & 154 & Salt & 9 & $9 / 5 / 96$ & 11250 & 60 & 94.3 & S96T004688 \\
\hline A-101 & 154 & Salt & 9 & $9 / 5 / 96$ & 11251 & 60 & 70.3 & S96T004687 \\
\hline A-101 & 154 & Salt & Comp & $10 / 2 / 96$ & 11562 & 250 & 35.2 & S96T005242 \\
\hline A-101 & 154 & Sait & Comp & $11 / 20 / 96$ & 11798 & 60 & 82.5 & S96T005244 \\
\hline & & & & & & Total Weight & 1766 & \\
\hline A-101 & 156 & $\mathrm{DL}$ & 10 & $8 / 21 / 96$ & 11080 & 250 & 90.7 & S96T004530 \\
\hline A-101 & 156 & $\mathrm{DL}$ & 10 & $8 / 27 / 96$ & 11341 & 60 & 71.9 & S96T004588 \\
\hline A-101 & 156 & $\mathrm{DL}$ & 11 & $8 / 14 / 96$ & 10633 & 250 & 209.2 & S96T004466 \\
\hline A-101 & 156 & $\mathrm{DL}$ & 11 & $8 / 27 / 96$ & 11353 & 60 & 93.9 & S96T004589 \\
\hline
\end{tabular}


Vials in 11A1 and 11A2

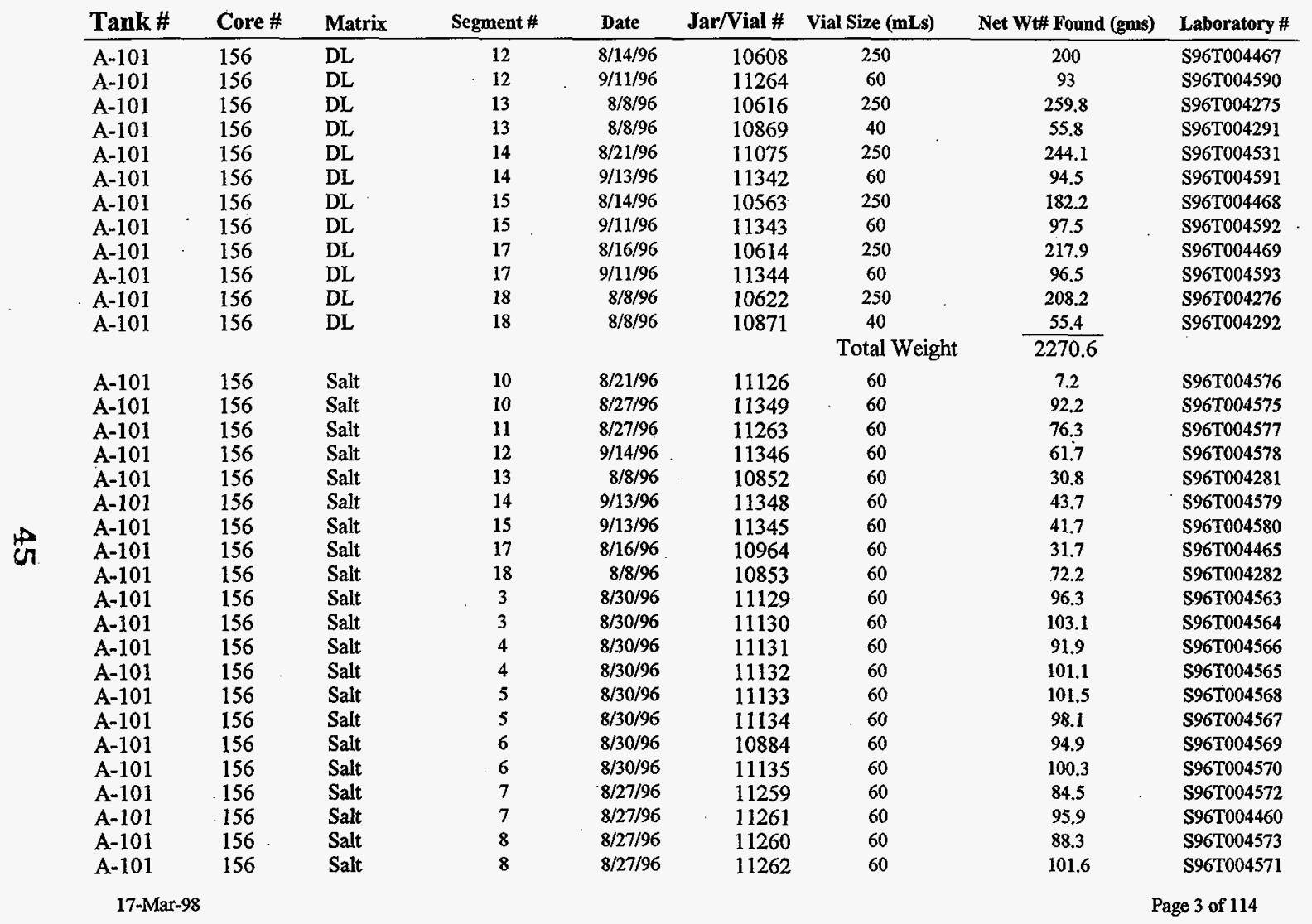


Vials in 11A1 and 11A2

\begin{tabular}{|c|c|c|c|c|c|c|c|c|}
\hline Tank \# & Core \# & Matrix & Segment \# & Date & Jar/Vial \# & Vial Size (mLs) & Net Wt\# Found (gms) & Laboratory \# \\
\hline & & & & & & Total Weight & 1615 & \\
\hline$A-101$ & 156 & Sludge & 16Rgs & & A101-16 & $\begin{array}{l}500 \\
\text { Total Weight }\end{array}$ & $\begin{array}{r}336.3 \\
336.3\end{array}$ & \\
\hline $\begin{array}{l}\text { A-101 } \\
\text { A-101 } \\
\text { A-101 }\end{array}$ & $\begin{array}{l}\text { Grab } \\
\text { Grab } \\
\text { Grab }\end{array}$ & $\begin{array}{l}\text { GL } \\
\text { GL } \\
\text { GL }\end{array}$ & $\begin{array}{l}96-1 \\
96-2 \\
96-3\end{array}$ & $\begin{array}{l}4 / 18 / 96 \\
4 / 18 / 96 \\
4 / 18 / 96\end{array}$ & $\begin{array}{l}9767 \\
9768 \\
9710\end{array}$ & $\begin{array}{c}\quad \begin{array}{c}40 \\
40 \\
60\end{array} \\
\text { Total Weight }\end{array}$ & $\begin{array}{c}51.5 \\
58.5 \\
79.8 \\
189.8\end{array}$ & $\begin{array}{l}\text { S96T002018 } \\
\text { S96T002019 } \\
\text { S96T002020 }\end{array}$ \\
\hline
\end{tabular}

\begin{tabular}{|c|c|c|c|c|c|c|c|c|}
\hline Tank \# & Core \# & Matrix & Segment \# & Date & Jar/Vial \# & Vial Size (mLs) & Net Wt\# Found (gms) & Laboratory \# \\
\hline AN-101 & Grab & GL & & $1 / 4 / 96$ & 8915 & 60 & 60.6 & S95T003904 \\
\hline AN-101 & Grab & GL & & $1 / 4 / 96$ & 8916 & 60 & 63.4 & S95T003907 \\
\hline AN-101 & Grab & GL & & $1 / 4 / 96$ & 8918 & 60 & 61.8 & S95T003908 \\
\hline AN-101 & Grab & GL & & $2 / 2 / 96$ & 9221 & 60 & 74 & S96T000339 \\
\hline AN-101 & Grab & GL & & $2 / 2 / 96$ & 9223 & 60 & 73.3 & S96T000340 \\
\hline AN-101 & Grab & GL & & $2 / 2 / 96$ & 9224 & 60 & 75.8 & S96T000341 \\
\hline & & & & & & Total Weight & 408.9 & \\
\hline
\end{tabular}

\begin{tabular}{|c|c|c|c|c|c|c|c|c|}
\hline Tank \# & Core \# & Matrix & Segment \# & Date & Jar/Vial \# & Vial Size (mLs) & Net Wt" Found (gms) & Laboratory \# \\
\hline AN-102 & 153 & FB & Fblk & $7 / 27 / 96$ & 10747 & $\begin{array}{c}40 \\
\text { Total Weight }\end{array}$ & $\frac{12.7}{12.7}$ & S96T003993 \\
\hline $\begin{array}{l}\text { AN-102 } \\
\text { AN-102 } \\
\text { AN-102 } \\
\text { AN-102 } \\
\text { AN-102 } \\
\text { AN-102 } \\
\text { AN-102 }\end{array}$ & $\begin{array}{l}\text { Grab } \\
\text { Grab } \\
\text { Grab } \\
\text { Grab } \\
\text { Grab } \\
\text { Grab } \\
\text { Grab }\end{array}$ & $\begin{array}{l}\text { GL } \\
\text { GL } \\
\text { GL } \\
\text { GL } \\
\text { GL } \\
\text { GL } \\
\text { GL }\end{array}$ & & $\begin{array}{r}1 / 5 / 96 \\
1 / 5 / 96 \\
1 / 11 / 96 \\
1 / 11 / 96 \\
1 / 11 / 96 \\
1 / 11 / 96 \\
2 / 5 / 98\end{array}$ & $\begin{array}{r}8919 \\
8920 \\
8967 \\
8968 \\
8969 \\
8970 \\
12958\end{array}$ & $\begin{array}{c}60 \\
60 \\
60 \\
60 \\
60 \\
60 \\
250 \\
\end{array}$ & $\begin{array}{c}54.3 \\
62.1 \\
72.2 \\
51.6 \\
58.2 \\
58.8 \\
180.1 \\
537.3\end{array}$ & $\begin{array}{l}\text { S95T003918 } \\
\text { S95T003919 } \\
\text { S95T004136 } \\
\text { S95T004132 } \\
\text { S95T004138 } \\
\text { S95T004134 }\end{array}$ \\
\hline
\end{tabular}


Vials in 11A1 and 11A2

\begin{tabular}{|c|c|c|c|c|c|c|c|c|}
\hline Tank \# & Core \# & Matrix & Segment \# & Date & Jar/Vial \# & Vial Size (mLs) & Net Wt\# Found (gms) & Laboratory \# \\
\hline AN-103 & 166 & $\mathrm{DL}$ & 10 & $10 / 21 / 96$ & 11528 & 250 & 225.9 & S96T005562 \\
\hline $\mathrm{AN}-103$ & 166 & DL & 10 & $10 / 23 / 96$ & 11418 & 40 & 57.5 & S96T005567 \\
\hline AN -103 & 166 & $\mathrm{DL}$ & 11 & $10 / 23 / 96$ & 11539 & 250 & 220.4 & \$96T005606 \\
\hline AN- 103 & 166 & $\mathrm{DL}$ & 11 & $11 / 9 / 96$ & 11423 & 40 & 57.9 & S96T005603 \\
\hline AN-103 & 166 & DL & 12 & $10 / 23 / 96$ & 11558 & 250 & 208.9 & S96T005607 \\
\hline AN-103 & 166 & $\overline{D L}$ & 12 & $11 / 9 / 96$ & 11424 & 40 & 59.2 & S96T005604 \\
\hline AN-103 & 166 & $\mathrm{DL}$ & 3 & $10 / 20 / 96$ & 11413 & 40 & 56 & S96T005536 \\
\hline AN-103 & 166 & $\overline{\mathrm{DL}}$ & 3 & $10 / 21 / 96$ & 11524 & 250 & 225.7 & S96T005531 \\
\hline AN-103 & 166 & $\overline{\mathrm{DL}}$ & 3 & $10 / 22 / 96$ & 11414 & 40 & 56 & S96T005537 \\
\hline AN-103 & 166 & $\mathrm{DL}$ & 4 & $10 / 21 / 96$ & 11529 & 250 & 221.8 & S96T005532 \\
\hline AN-103 & 166 & $\mathrm{DL}$ & 6 & $10 / 23 / 96$ & 11537 & 250 & 225.9 & S96T005605 \\
\hline AN-103 & 166 & DL & 6 & $11 / 8 / 96$ & 11422 & 40 & 57.8 & S96T005602 \\
\hline AN-103 & 166 & $\mathrm{DL}$ & 7 & $10 / 31 / 96$ & 11543 & 250 & 236.6 & S96T005814 \\
\hline AN-103 & 166 & $\mathrm{DL}$ & 7 & $11 / 10 / 96$ & 11425 & 40 & .57 .9 & S96T005818 \\
\hline AN-103 & 166 & DL & 8 & $10 / 31 / 96$ & 11542 & 250 & 251.2 & S96T005815 \\
\hline AN-103 & 166 & DL & 8 & $11 / 10 / 96$ & 11426 & 40 & 57.7 & S96T005819 \\
\hline$A N-103$ & 166 & $\mathrm{DL}$ & 9 & $10 / 21 / 96$ & 11519 & 250 & 227.6 & S96T005533 \\
\hline AN-103 & 166 & $\mathrm{DL}$ & 9 & $10 / 22 / 96$ & 11415 & 40 & 56.2 & S96T005538 \\
\hline \multirow[t]{2}{*}{ AN-103 } & 166 & $\mathrm{DL}$ & Comp & $12 / 11 / 96$ & 11896 & 60 & 34.7 & S96T005994 \\
\hline & & & & & & Total Weight & 2594.9 & \\
\hline AN-103 & 166 & FB & Folk & $10 / 7 / 96$ & 11400 & 250 & 165.5 & \$96T005368 \\
\hline \multirow[t]{2}{*}{ AN-103 } & 166 & $\mathrm{FB}$ & Fblk & $10 / 20 / 96$ & 11411 & 40 & 37 & S96T005407 \\
\hline & & & & & & Total Weight & 202.5 & \\
\hline AN-103 & 166 & Salt & 1 & $11 / 8 / 96$ & 11775 & 60 & 21.7 & S96T005590 \\
\hline AN-103 & 166 & Salt & 10 & $10 / 21 / 96$ & 11618 & 60 & $35: 3$ & S96T005565 \\
\hline$A N-103$ & 166 & Salt & 11 & $10 / 23 / 96$ & 11624 & 60 & 21.3 & S96T005580 \\
\hline AN-103 & 166 & Salt & 12 & $11 / 9 / 96$ & 11776 & 60 & 18 & S96T005593 \\
\hline AN-103 & 166 & Salt & 13 & $10 / 24 / 96$ & 11515 & 250 & 105 & S $96 \mathrm{~T} 005629$ \\
\hline AN-103 & 166 & Salt & 13 & $10 / 24 / 96$ & 11555 & 250 & 79.7 & S96T005632 \\
\hline AN-103 & 166 & Salt & 13 & $10 / 25 / 96$ & 11631 & 60 & 89.5 & S96T005640 \\
\hline AN-103 & 166 & Salt & 13 & $10 / 25 / 96$ & 11634 & 60 & 92.1 & S96T005641 \\
\hline AN-103 & 166 & Salt & 15 & $10 / 7 / 96$ & 11396 & 250 & 92.7 & S96T005371 \\
\hline AN-103 & 166 & Salt & 15 & $10 / 7 / 96$ & 11402 & 250 & 93.3 & S96T005369 \\
\hline
\end{tabular}


Vials in 11A1 and 11A2

\begin{tabular}{|c|c|c|c|c|c|c|c|c|}
\hline Tank \# & Core\# & Matrix & Segment \# & Date & Jar/Vial \# & Vial Size (mLs) & Net Wt\# Found (gms) & Laboratory \# \\
\hline$A N-103$ & 166 & Salt & 15 & $10 / 20 / 96$ & 11508 & 60 & 94.6 & S96T005402 \\
\hline AN-103 & 166 & Salt & 15 & $10 / 20 / 96$ & 11509 & 60 & 104.3 & S96T005403 \\
\hline AN-103 & 166 & Salt & 16 & $10 / 23 / 96$ & 11531 & 250 & 70.8 & S96T005588 \\
\hline$A N-103$ & 166 & Salt & 16 & $10 / 23 / 96$ & 11533 & 250 & 81.9 & S96T005589 \\
\hline AN-103 & 166 & Salt & 16 & $11 / 9 / 96$ & 11777 & 60 & 108.1 & S96T005594 \\
\hline AN-103 & 166 & Salt & 16 & $11 / 9 / 96$ & 11778 & 60 & 101.3 & S96T005595 \\
\hline AN-103 & 166 & Salt & 17 & $10 / 7 / 96$ & 11194 & 250 & 65.2 & S96T005372 \\
\hline AN-103 & 166 & Salt & 17 & $10 / 7 / 96$ & 11570 & 250 & 85.9 & \$96T005370 \\
\hline AN-103 & 166 & Salt & 17 & $10 / 20 / 96$ & 11510 & 60 & 81.1 & S96T005404 \\
\hline AN-103 & 166 & Salt & 17 & $10 / 20 / 96$ & 11511 & 60 & 91.7 & S96T005405 \\
\hline AN-103 & 166 & Salt & 18 & $10 / 24 / 96$ & 11538 & 250 & 73 & S96T005631 \\
\hline AN-103 & 166 & Salt & 18 & $10 / 24 / 96$ & 11553 & 250 & 66.2 & S96T005634 \\
\hline AN-103 & 166 & Salt & 18 & $10 / 25 / 96$ & 11635 & 60 & 108.2 & S96T005642 \\
\hline AN-103 & 166 & Salt & 18 & $10 / 25 / 96$ & 11636 & 60 & 98.1 & S96T005643 \\
\hline $\mathrm{AN}-103$ & 166 & Salt & 19 & $10 / 31 / 96$ & 11541 & 250 & 83.3 & S96T005812 \\
\hline AN-103 & 166 & Salt & 19 & $10 / 31 / 96$ & 11545 & 250 & 71.1 & S96T005813 \\
\hline$A N-103$ & 166 & Salt & 19 & $11 / 10 / 96$ & 11781 & 60 & 93.2 & S96T005826 \\
\hline AN-103 & 166 & Salt & 19 & $11 / 10 / 96$ & 11782 & 60 & 77.3 & S96T005827 \\
\hline AN-103 & 166 & Salt & 4 & $10 / 21 / 96$ & 11616 & 60 & 41.8 & S96T005544 \\
\hline AN-103 & 166 & Salt & 6 & $10 / 23 / 96$ & 11623 & 60 & 36.5 & S96T005585 \\
\hline AN-103 & 166 & Salt & 7 & $11 / 10 / 96$ & 11779 & 60 & 27.1 & S96T005824 \\
\hline AN-103 & 166 & Salt & 8 & $11 / 10 / 96$ & 11780 & 60 & 12.1 & \$96T005825 \\
\hline AN-103 & 166 & Salt & 9 & $10 / 21 / 96$ & 11617 & 60 & 35.5 & S96T005545 \\
\hline AN-103 & 166 & Salt & Comp & & 11940 & 250 & 88.3 & S96T005983 \\
\hline \multirow[t]{2}{*}{ AN-103 } & 166 & Salt & Comp & $12 / 14 / 96$ & 11813 & 60 & 94.8 & S96T005935 \\
\hline & & & & & & Total Weight & 2540 & \\
\hline AN-103 & 167 & DL & 11 & $11 / 13 / 96$ & 11957 & 40 & 24.5 & S96T005652 \\
\hline AN-103 & 167 & DL & 2 & $10 / 31 / 96$ & 11546 & 250 & 183.1 & S96T005849 \\
\hline AN-103 & 167 & DL & 2 & $11 / 11 / 96$ & 11427 & 40 & 56 & S96T005863 \\
\hline AN-103 & 167 & DL & 3 & $10 / 28 / 96$ & 11556 & 250 & 298.5 & S96T005664 \\
\hline$A N-103$ & 167 & $\overline{\mathrm{DL}}$ & 3 & $11 / 13 / 96$ & 11954 & 40 & 55.8 & S96T005674 \\
\hline AN-103 & 167 & $\mathrm{DL}$ & 5 & $10 / 7 / 96$ & 11569 & 250 & 274.5 & S96T005373 \\
\hline AN-103 & 167 & DL & 5 & $10 / 21 / 96$ & 11417 & 40 & 48.5 & \$96T005422 \\
\hline AN-103 & 167 & DL & 6 & $10 / 7 / 96$ & 11525 & 250 & 287.1 & S96T005380 \\
\hline \multicolumn{3}{|c|}{ 17-Mar-98 } & & . & & & \multicolumn{2}{|c|}{ Page 6 of 114} \\
\hline
\end{tabular}


Vials in 11A1 and 11A2

\begin{tabular}{|c|c|c|c|c|c|c|c|c|}
\hline Tank \# & Core \# & Matrix & Segment \# & Date & Jar/Vial \# & Vial Size (mLs) & Net Wt\# Found (gms) & Laboratory \# \\
\hline $\mathrm{AN}-103$ & 167 & DL & 6 & $10 / 21 / 96$ & 11416 & 40 & 31.9 & $\$ 96 \mathrm{~T} 005423$ \\
\hline AN-103 & 167. & DL & 7 & $11 / 15 / 96$ & 11956 & 40 & 35.8 & \$96T005682 \\
\hline AN-103 & 167 & DL & 8 & $10 / 29 / 96$ & 11534 & 250 & 228.5 & S96T005731 \\
\hline $\mathrm{AN}-103$ & 167 & DL & 9 & $10 / 31 / 96$ & 11560 & 250 & 294.4 & S96T005850 \\
\hline \multirow[t]{2}{*}{$\mathrm{AN}-103$} & 167 & DL & 9 & $11 / 11 / 96$ & 11942 & 40 & 57 & \multirow[t]{2}{*}{ S96T005864 } \\
\hline & & & & & & Total Weight & 1875.6 & \\
\hline AN-103 & 167 & Salt & 1 & $10 / 28 / 96$ & 11536 & 250 & 42.2 & S96T005661 \\
\hline AN-103 & 167 & Salt & 1 & $11 / 13 / 96$ & 11785 & 60 & 93.9 & S96T001178 \\
\hline AN-103 & 167 & Salt & 1 & $11 / 13 / 96$ & 11786 & 60 & 108.3 & S96T005671 \\
\hline $\mathrm{AN}-103$ & 167 & Salt & 11 & $10 / 24 / 96$ & 11554 & 250 & 91 & S96T005644 \\
\hline $\mathrm{AN}-103$ & 167 & Salt & 11 & $10 / 24 / 96$ & 11557 & 250 & 41.4 & S96T005377 \\
\hline AN-103 & 167 & Salt & 11 & $11 / 13 / 96$ & 11788 & 60 & 104.1 & S96T005648 \\
\hline AN-103 & 167 & Salt & 11 & $11 / 13 / 96$ & 11789 & 60 & 104.9 & S96T005649 \\
\hline $\mathrm{AN}-103$ & 167 & Salt & 12 & & 11503 & 60 & 114.9 & S96T005434 \\
\hline$A N-103$ & 167 & Salt & 12 & $10 / 2 / 96$ & 11393 & 250 & 118.3 & S96T005378 \\
\hline AN-103 & 167 & Salt & 12 & $10 / 22 / 96$ & 11502 & 60 & 113 & S96T005430 \\
\hline AN-103 & 167 & Salt & 14 & $10 / 23 / 96$ & 11504 & 60 & 110.9 & S96T005435 \\
\hline AN-103 & 167 & Salt & 14. & $10 / 23 / 96$ & 11512 & 60 & 126 & S96T005431 \\
\hline AN-103 & 167 & Salt & 15 & $10 / 11 / 96$ & 11518 & 250 & 64 & S96T005653 \\
\hline AN-103 & 167 & Salt & 15 & $10 / 11 / 96$ & 11527 & 250 & 49.4 & S96T005654 \\
\hline AN-103 & 167 & Salt & 15 & $11 / 18 / 96$ & 11790 & 60 & 131 & S96T005657 \\
\hline AN-103 & 167 & Salt & 15 & $11 / 18 / 96$ & 11791 & 60 & 123.4 & S96T005658 \\
\hline AN-103 & 167 & Salt & 17 & $10 / 23 / 96$ & 11622 & 60 & 117.5 & S96T005494 \\
\hline AN-103 & 167 & Salt & 17 & $10 / 24 / 96$ & 11621 & 60 & 97.2 & \$96T005495 \\
\hline AN-103 & 167 & Salt & 2 & $11 / 11 / 96$ & 11783 & 60 & 76.8 & S96T005853 \\
\hline AN-103 & 167 & Salt & 3 & $10 / 28 / 96$ & 11630 & 60 & 20.2 & S96T005672 \\
\hline AN-103 & 167 & Salt & 4 & $10 / 28 / 96$ & 11632 & 60 & 19.6 & S96T005676 \\
\hline$A N-103$ & 167 & Salt & 5 & $10 / 22 / 96$ & 11500 & 60 & 50.9 & S96T005428 \\
\hline AN- 103 & 167 & Salt & 6 & $10 / 22 / 96$ & 11485 & 60 & 40.3 & S96T005429 \\
\hline AN-103 & 167 & Salt & 7 & $10 / 28 / 96$ & 11638 & 60 & 32 & S96T005684 \\
\hline AN-103 & 167 & Salt & 8 & $10 / 29 / 96$ & 11759 & 60 & 34.3 & S96T005730 \\
\hline \multirow[t]{2}{*}{$\mathrm{AN}-103$} & 167 & Salt & 9 & $11 / 11 / 96$ & 11784 & 60 & 43.1 & S96T005854 \\
\hline & & & & & & Total Weight & 2068.6 & \\
\hline
\end{tabular}


Vials in 11A1 and 11A2

\begin{tabular}{|c|c|c|c|c|c|c|c|c|}
\hline Tank \# & Core \# & Matrix & Segment \# & Date & Jar/Vial \# & Vial Size (mLs) & Net Wt\# Found (gms) & Laboratory \# \\
\hline AN-104 & 163 & $\mathrm{DL}$ & 10 & $11 / 5 / 96$ & 11420 & 40 & 53.7 & \$96T005530 \\
\hline AN-104 & 163 & DL & 11 & $10 / 25 / 96$ & 11551 & 250 & 172.3 & S96T005726 \\
\hline AN-104 & 163 & DL & 11 & $11 / 5 / 96$ & 11421 & 40 & 57.3 & S96T005740 \\
\hline AN-104 & 163 & $\mathrm{DL}$ & 12 & $10 / 30 / 96$ & 11547 & 250 & 180.8 & S96T005765 \\
\hline AN-104 & 163 & DL & 12 & $11 / 8 / 96$ & 11768 & 60 & 95 & S96T005767 \\
\hline AN-104 & 163 & DL & 14 & $10 / 25 / 96$ & 11628 & 60 & 33.2 & S96T005727 \\
\hline AN-104 & 163 & $\mathrm{DL}$ & 2 & $9 / 25 / 96$ & 11188 & 250 & 187.7 & S96T005224 \\
\hline $\mathrm{AN}-104$ & 163 & DL & 2 & $10 / 12 / 96$ & 11404 & 40 & 51.8 & S96T005266 \\
\hline AN-104 & 163 & DL & 21 & $10 / 21 / 96$ & 11526 & 250 & 162.6 & S96T005526 \\
\hline $\mathrm{AN}-104$ & 163 & $\mathrm{DL}$ & 3Rgs & & AN104-3 & 500 & 29.2 & \\
\hline AN-104 & 163 & $\mathrm{DL}$ & 4 & 9/25/96 & 11189 & 250 & 141.3 & S96T005229 \\
\hline AN-104 & 163 & DL & 4 & $10 / 12 / 96$ & 11405 & 40 & 51 & S96T005261 \\
\hline$A N-104$ & 163 & $\mathrm{DL}$ & 5 & $10 / 21 / 96$ & 11523 & 250 & 179.5 & S96T005525 \\
\hline AN-104 & 163 & DL & 5 & $11 / 5 / 96$ & 11762 & 60 & 98.5 & S96T005529 \\
\hline AN-104 & 163 & $\mathrm{DL}$ & 6 & $10 / 10 / 96$ & 11522 & 250 & 173 & S96T005547 \\
\hline AN-104 & 163 & DL & 6 & $11 / 5 / 96$ & 11412 & 40 & 52.4 & S96T005555 \\
\hline AN-104 & 163 & DL & 7 & $9 / 25 / 96$ & 11192 & 250 & 210.8 & S96T005230 \\
\hline AN-104 & 163 & DL & 7 & $10 / 12 / 96$ & 11406 & 40 & 54 & S96T005262 \\
\hline $\mathrm{AN}-104$ & 163 & DL & 8 & $9 / 26 / 96$ & 11195 & 250 & 124 & S96T005231 \\
\hline AN-104 & 163 & DL & 8 & $10 / 12 / 96$ & 11407 & 40 & 55.7 & S96T005263 \\
\hline AN-104 & 163 & DL & 9 & 9/26/96 & 11196 & 250 & 112.9 & S96T005232 \\
\hline \multirow[t]{2}{*}{ AN-104 } & 163 & DL & 9 & $10 / 13 / 96$ & 11408 & 40 & 55.8 & S96T005265 \\
\hline & & & & & & Total Weight & 2332.5 & \\
\hline AN-104 & 163 & Salt & 1 & $9 / 25 / 96$ & 11373 & 60 & 12.3 & S96T005228 \\
\hline AN-104 & 163 & Salt & 12 & $11 / 7 / 96$ & 11769 & 60 & 56.5 & S96T005774 \\
\hline AN-104 & 163 & Salt & 14 & $10 / 25 / 96$ & 11514 & 250 & 48.5 & S96T005728 \\
\hline AN-104 & 163 & Salt & 14 & $10 / 25 / 96$ & 11559 & 250 & 53.2 & S96T005729 \\
\hline AN-104 & 163 & Salt & 14 & $11 / 5 / 96$ & 11765 & 60 & 97.1 & S96T005746 \\
\hline AN-104 & 163 & Salt & 14 & $11 / 6 / 96$ & 11764 & 60 & 95.6 & S96T005745 \\
\hline AN-104 & 163 & Salt & 16 & $9 / 26 / 96$ & 11197 & 250 & 76.6 & \$96T005227 \\
\hline AN-104 & 163 & Salt & 16 & $9 / 26 / 96$ & 11198 & 250 & 106.1 & S96T005226 \\
\hline AN-104 & 163 & Salt & 16 & $10 / 14 / 96$ & 11494 & 60 & 87.8 & S96T005276 \\
\hline AN-104 & 163 & Salt & 16 & $10 / 14 / 96$ & 11495 & 60 & 82.2 & S96T005275 \\
\hline AN-104 & 163 & Salt & 18 & $10 / 30 / 96$ & 11544 & 250 & 99.6 & S96T005769 \\
\hline 17-Mar-S & & & & & & & & ge 8 of 114 \\
\hline
\end{tabular}


Vials in 11A1 and 11A2

\begin{tabular}{|c|c|c|c|c|c|c|c|c|}
\hline Tank \# & Core \# & Matrix & Segment \# & Date & Jar/Vial \# & Vial Size (mLs) & Net Wt\# Found (gms) & Laboratory \# \\
\hline $\mathrm{AN}-104$ & 163 & Salt & 18 & $10 / 30 / 96$ & 11549 & 250 & 70.5 & S96T005770 \\
\hline AN-104 & 163 & Salt & 18 & $11 / 8 / 96$ & 11770 & 60 & 91.7 & S96T005775 \\
\hline AN-104 & 163 & Salt & 18 & $11 / 8 / 96$ & 11771 & 60 & 97.3 & S96T005776 \\
\hline $\mathrm{AN}-104$ & 163 & Salt & 2 & $9 / 25 / 96$ & 11374 & 60 & 18.3 & S96T005225 \\
\hline AN-104 & 163 & Salt. & 20 & $10 / 2 / 96$ & 11397 & 250 & 84.1 & S96T005553 \\
\hline AN-104 & 163 & Salt & 20 & $10 / 2 / 96$ & 11398 & 250 & 65 & S96T005552 \\
\hline AN-104 & 163 & Salt & 20 & $11 / 6 / 96$ & 11766 & 60 & 99 & S96T005551 \\
\hline \multirow[t]{2}{*}{ AN-104 } & 163 & Salt & 20 & $11 / 6 / 96$ & 11767 & 60 & 102 & S96T005550 \\
\hline & & & & & & Total Weight & 1443.4 & \\
\hline$A N-104$ & 163 & Sludge & 13-Rgs & & AN104-13 & 500 & 15.3 & \\
\hline AN-104 & 163 & Sludge & 15-RGS & & AN104-15 & 500 & 11.9 & \\
\hline AN-104 & 163 & Sludge & 17Rgs & & AN104-17 & 500 & 119.6 & \\
\hline AN-104 & 163 & Sludge & 18-Rgs . & & AN104-18 & 500 & 7 & \\
\hline AN-104 & 163 & Sludge & 19Rgs & & AN104-19 & 500 & 37.9 & \\
\hline AN-104 & 163 & Sludge & Commp & $1 / 26 / 98$ & 14064 & 500 & 701.1 & S97M000285 \\
\hline AN-104 & 163 & Sludge & Comp & $1 / 26 / 98$ & 12951 & 250 & 113.5 & \\
\hline \multirow[t]{2}{*}{ AN-104 } & 163 & Sludge & Comp & $1 / 26 / 98$ & 14066 & 500 & 632.1 & S97M000284 \\
\hline & & & & & & Total Weight & 1638.4 & \\
\hline AN-104 & 164 & DL & 1 & $8 / 28 / 96$ & 11164 & 250 & 186.6 & S96T004762 \\
\hline AN-104 & 164 & DL & 1 & $9 / 14 / 96$ & 11103 & 40 & 54.7 & S96T004775 \\
\hline AN-104 & 164 & $\mathrm{DL}$ & 10 & $9 / 11 / 96$ & 11185 & 250 & 256.5 & S96T004964 \\
\hline AN-104 & 164 & $\mathrm{DL}$ & 10 & $9 / 16 / 96$ & 11115 & 40 & 53.2 & S96T004980 \\
\hline AN-104 & 164 & $\mathrm{DL}$ & 11 & $8 / 29 / 96$ & 11094 & 250 & 322.3 & N/A \\
\hline AN-104 & 164 & DL & 11 & $9 / 14 / 96$ & 11108 & 40 & 51.8 & S96T004789 \\
\hline AN-104 & 164 & DL & 12 & $8 / 29 / 96$ & 11095 & 250 & 299.6 & N/A \\
\hline $\mathrm{AN}-104$ & 164 & $\overline{D L}$ & 12 & $9 / 3 / 96$ & 11109 & 40 & 55.9 & S96T004790 \\
\hline AN-104 & 164 & $\mathrm{DL}$ & 13 & $8 / 29 / 96$ & 11097 & 250 & 225.1 & S96T004770 \\
\hline AN-104 & 164 & $\mathrm{DL}$ & 13 & 9/3/96 & 11110 & 40 & 53.4 & S96T004791 \\
\hline AN-104 & 164 & $\mathrm{DL}$ & 2 & $8 / 28 / 96$ & 11081 & 250 & 288.8 & S96T004764 \\
\hline AN-104 & 164 & DL & 2 & $9 / 14 / 96$ & 11104 & 40 & 51.6 & S96T004785 \\
\hline AN-104 & 164 & DL & 3 & $8 / 28 / 96$ & 11091 & 250 & 293.2 & S96T004765 \\
\hline AN-104 & 164 & $\mathrm{DL}$ & 3 & $9 / 14 / 96$ & 11105 & 40 & 52.1 & S96T004779 \\
\hline AN-104 & 164 & $\mathrm{DL}$ & 4 & $9 / 14 / 96$ & 11106 & 40 & 51.5 & S96T004787 \\
\hline
\end{tabular}


Vials in 11A1 and 11A2

\begin{tabular}{|c|c|c|c|c|c|c|c|c|}
\hline Tank \# & Core \# & Matrix & Segment \# & Date & Jar/Vial \# & Vial Size (mLs) & Net Wt\# Found (gms) & Laboratory \# \\
\hline AN-104 & 164 & DL & 4 & $12 / 6 / 96$ & 11717 & 125 & 132.6 & S96T004766 \\
\hline AN-104 & 164 & DL & 5 & 9/9/96 & 10977 & 125 & 27.8 & S96T004961 \\
\hline AN-104 & 164 & DL & 5 . & 9/9/96 & 11083 & 250 & 337.6 & N/A \\
\hline AN-104 & 164 & $\mathrm{DL}$ & 5 & $9 / 16 / 96$ & 11112 & 40 & 52.7 & S96T004983 \\
\hline AN-104 & 164 & DL & 7 & $8 / 29 / 96$ & 11092 & 250 & 347 & N/A \\
\hline AN-104 & 164 & DL & 7 & $9 / 14 / 96$ & 11107 & 40 & 51.7 & S96T004788 \\
\hline AN-104 & 164 & DL & 8 & 9/9/96 & 11175 & 250 & 318.3 & N/A \\
\hline AN-104 & 164 & DL & 8 & $9 / 16 / 96$ & 11113 & 40 & 51.3 & S96T004982 \\
\hline AN-104 & 164 & $\overline{\mathrm{DL}}$ & 9 & $9 / 16 / 96$ & 11114 & 40 & 38.5 & S $96 \mathrm{~T} 004981$ \\
\hline AN-104 & 164 & $\overline{D L}$ & Comp & $12 / 4 / 96$ & 11561 & 250 & 77.2 & S96T005971 \\
\hline \multirow[t]{2}{*}{ AN-104 } & 164 & DL & Comp & $12 / 8 / 96$ & 11811 & 60 & 41.1 & S96T005982 \\
\hline & & & & & & Total Weight & 3772.1 & \\
\hline AN-104 & 164 & FB & Folk & $9 / 24 / 96$ & 11180 & 250 & 125.8 & S96T005221 \\
\hline \multirow[t]{2}{*}{ AN-104 } & 164 & FB & Folk & $10 / 14 / 96$ & 11409 & 40 & 31.1 & S96T005254 \\
\hline & & & & & & Total Weight & 156.9 & \\
\hline AN-104 & 164 & Salt & 1 & $8 / 28 / 96$ & 10965 & 60 & 25.7 & S96T004763 \\
\hline AN-104 & 164 & Salt & 13 & $9 / 3 / 96$ & 11347 & 60 & 30.4 & S96T004777 \\
\hline AN-104 & .164 & Salt & 14 & 9/18/96 & 11368 & 60 & 105.9 & S96T004994 \\
\hline AN-104 & 164 & Salt & 14 & 9/18/96 & 11369 & 60 & 71 & S96T004995 \\
\hline AN-104 & 164 & Salt & 15 & $9 / 20 / 96$ & 11370 & 60 & 75.9 & S96T004996 \\
\hline AN-104 & 164 & Salt & 15 & $9 / 20 / 96$ & 11376 & 60 & 81.9 & S96T004997 \\
\hline AN-104 & 164 & Salt & 16 & $9 / 20 / 96$ & 11377 & 60 & 86.8 & S96T004998 \\
\hline AN-104 & 164 & Salt & 16 & $9 / 21 / 96$ & 11378 & 60 & 81.3 & S96T004999 \\
\hline AN-104 & 164 & Salt & 17 & $9 / 21 / 96$ & 11379 & 60 & 68.2 & S96T005000 \\
\hline AN-104 & 164 & Salt & 17 & $9 / 21 / 96$ & 11380 & 60 & 87.8 & S96T005001 \\
\hline AN-104 & 164 & Salt & 19 & $9 / 21 / 96$ & 11381 & 60 & 85.2 & S96T005002 \\
\hline AN-104 & 164 & Salt & 19 & $9 / 21 / 96$ & 11382 & 60 & 100 & S96T005003 \\
\hline AN-104 & 164 & Salt & 20 & $9 / 25 / 96$ & 11190 & 250 & 81.6 & S96T005222 \\
\hline AN-104 & 164 & Salt & 20 & $10 / 14 / 96$ & 11497 & 60 & 69.2 & S96T005251 \\
\hline AN-104 & 164 & Salt & 20 & $10 / 14 / 96$ & 11499 & 60 & 86.7 & S96T005252 \\
\hline \multirow[t]{2}{*}{ AN-104 } & 164 & Salt & Comp & $12 / 8 / 96$ & 11812 & 60 & 74.4 & S96T005973 \\
\hline & & & & & & Total Weight & 1212 & \\
\hline
\end{tabular}


Vials in 11A1 and 11A2

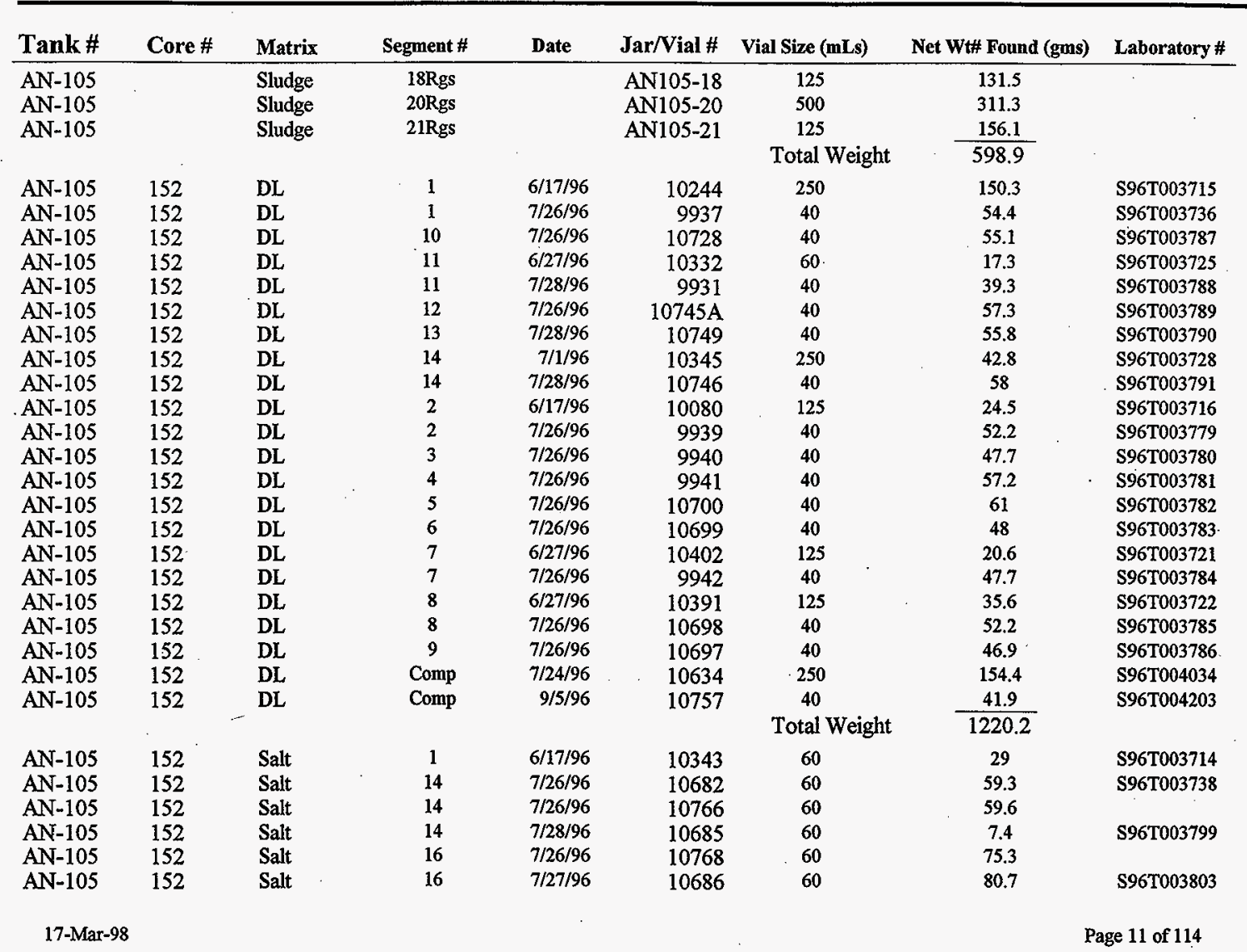


Vials in 11A1 and 11A2

\begin{tabular}{|c|c|c|c|c|c|c|c|c|}
\hline Tank \# & Core \# & Matrix & Segment \# & Date & Jar/Vial \# & Vial Size (mLs) & Net Wt\# Found (gms) & Laboratory \# \\
\hline AN-105 & 152 & Salt & 16 & $7 / 27 / 96$ & 10687 & 60 & 77.8 & S96T003810 \\
\hline AN-105 & 152 & Salt & 18 & & 10688 & 60 & 26.6 & S96T003804 \\
\hline $\mathrm{AN}-105$ & 152 & Salt & 18 & & 10690 & 60 & 61.2 & S96T003811 \\
\hline AN- 105 & 152 & Salt & 20 & & 10691 & 60 & 59 & S96T003805 \\
\hline$A N-105$ & 152 & Salt & 20 & & 10692 & 60 & 42.6 & S96T003812 \\
\hline AN-105 & 152 & Salt & 20 & $7 / 27 / 96$ & 10770 & 60 & 72.7 & \\
\hline AN-105 & 152 & Salt & 22 & $7 / 27 / 96$ & 10769 & 60 & 75.3 & . \\
\hline $\mathrm{AN}-105$ & 152 & Salt & 22 & $8 / 17 / 96$ & 10694 & 60 & 13.8 & S96T003806 \\
\hline AN-105 & 152 & Salt & 22 & $8 / 17 / 96$ & 11121 & 60 & 66.8 & S96T004260 \\
\hline AN-105 & 152 & Salt & Comp & $8 / 17 / 96$ & 10775 & 60 & 41.1 & \\
\hline \multirow[t]{2}{*}{ AN- 105} & 152 & Salt & Comp & $8 / 17 / 96$ & 11120 & 60 & 84.8 & S96T004255 \\
\hline & & & & & & Total Weight & 933 & \\
\hline AN-105 & 153 & DL & 1 & $8 / 14 / 96$ & 10609 & 250 & 176.3 & S96T004425 \\
\hline AN-105 & 153 & $\overrightarrow{\mathrm{DL}}$ & 1 & $8 / 19 / 96$ & 11100 & 40 & 53.4 & S96T004431 \\
\hline AN-105 & 153 & DL & 10 & $8 / 5 / 96$ & 10795 & 125 & 42.8 & S96T004309 \\
\hline $\mathrm{AN}-105$ & 153 & DL & 10 & $8 / 9 / 96$ & 10874 & 40 & 54.8 & S96T004307 \\
\hline AN-105 & 153 & DL & 11 & $7 / 28 / 96$ & 10751 & 40 & 44.1 & \$96T003996 \\
\hline AN-105 & 153 & $\overline{\mathrm{DL}}$ & 12 & $7 / 28 / 96$ & 10752 & 40 & 40.6 & S96T003997 \\
\hline AN-105 & 153 & $\overline{\mathrm{DL}}$ & 13 & $7 / 28 / 96$ & 10753 & 40 & 45.7 & S96T003998 \\
\hline AN-105 & 153 & $\mathrm{DL}$ & 14 & $8 / 7 / 96$ & 10794 & 125 & 60.7 & S96T004331 \\
\hline AN-105 & 153 & DL & 14 & $8 / 16 / 96$ & 11098 & 40 & 56.2 & S $96 \mathrm{~T} 004332$ \\
\hline AN-105 & 153 & DL & 2 & $8 / 9 / 96$ & 10793 & 125 & 31.9 & S96T004305 \\
\hline AN-105 & 153 & DL & 2 & $8 / 9 / 96$ & 10872 & 40 & 53.7 & S96T004306 \\
\hline$A N-105$ & 153 & DL & 21 & $7 / 9 / 96$ & 10523 & 125 & 110.9 & S96T003970 \\
\hline $\mathrm{AN}-105$ & 153 & DL & 21 & $7 / 28 / 96$ & 10754 & 40 & 48.5 & S96T003999 \\
\hline AN-105 & 153 & DL & 3 & $8 / 19 / 96$ & 11101 & 40 & 51.1 & S96T004432 \\
\hline$A N-105$ & 153 & DL & 5 & $8 / 12 / 96$ & 10790 & 125 & 66.5 & S96T004413 \\
\hline AN- 105 & 153 & DL & 5 & $8 / 16 / 96$ & 10875 & 40 & 55.1 & S96T004416 \\
\hline AN-105 & 153 & $\overline{\mathrm{DL}}$ & 6 & $8 / 12 / 96$ & 10785 & 125 & 67.8 & S96T004414 \\
\hline AN-105 & 153 & $\overline{D L}$ & 6 & $8 / 16 / 96$ & 10876 & 40 & 54.2 & S96T004417 \\
\hline AN-105 & 153 & $\mathrm{DL}$ & 7 & $8 / 12 / 96$ & 10789 & 125 & 25.7 & S96T004415 \\
\hline AN-105 & 153 & DL & 7 & $8 / 16 / 96$ & 10877 & 40 & 49 & S96T004418 \\
\hline AN-105 & 153 & DL & 8 & $7 / 28 / 96$ & 10748 & 40 & 53.6 & S96T003994 \\
\hline AN-105 & 153 & DL & 9 & $7 / 8 / 96$ & 10400 & 125 & 24.3 & S96T003966 \\
\hline \multicolumn{3}{|c|}{ 17-Mar-98 } & & & & & & ge 12 of 114 \\
\hline
\end{tabular}


Vials in 11A1 and 11A2

\begin{tabular}{|c|c|c|c|c|c|c|c|c|}
\hline Tank \# & Core \# & Matrix & Segment \# & Date & Jar/Vial \# & Vial Size (mLs) & Net Wt\# Found (gms) & Laboratory \# \\
\hline AN-105 & 153 & $\mathrm{DL}$ & 9 & $7 / 28 / 96$ & 10750 & $\begin{array}{c}40 \\
\text { Total Weight }\end{array}$ & $\frac{53.1}{1320}$ & S96T003995 \\
\hline AN-105 & 153 & FB & Folk & $7 / 9 / 96$ & 10522 & $\begin{array}{l}250 \\
\text { Total Weight }\end{array}$ & $\begin{array}{r}107.5 \\
107.5\end{array}$ & S96T003964 \\
\hline AN-105 & 153 & Salt & 14 & $8 / 16 / 96$ & 10967 & 60 & 81.9 & S96T004336 \\
\hline AN-105 & 153 & Salt & 15 & $8 / 5 / 96$ & 10602 & 250 & 66.9 & S96T004303 \\
\hline AN-105 & 153 & Salt & 15 & $8 / 9 / 96$ & 10857 & 60 & 100.1 & S96T004313 \\
\hline AN-105 & 153 & Salt & 17 & $8 / 19 / 96$ & 11122 & 60 & 79.5 & S96T004439 \\
\hline AN-105 & 153 & Salt & 17 & $8 / 19 / 96$ & 11123 & 60 & 83.2 & S96T004438 \\
\hline $\mathrm{AN}-105$ & 153 & Salt & 19 & $8 / 19 / 96$ & 11124 & 60 & 89.6 & S96T004440 \\
\hline AN-105 & 153 & Salt & 21 & $7 / 28 / 96$ & 10761 & 60 & 87.7 & S96T004000 \\
\hline$A N-105$ & 153 & Salt & 22 & $7 / 28 / 96$ & 10762 & 60 & 73 & S96T004001 \\
\hline AN-105 & 153 & Salt & 22 & $7 / 28 / 96$ & 10763 & $\begin{array}{l}60 \\
\text { Total Weight }\end{array}$ & $\begin{array}{c}87.7 \\
749.6\end{array}$ & S96T004002 \\
\hline AN-105 & 153 & Sludge & 15 & $8 / 9 / 96$ & 10854 & $\begin{array}{l}60 \\
\text { Total Weight }\end{array}$ & $\frac{94.5}{94.5}$ & S96T004312 \\
\hline
\end{tabular}

\begin{tabular}{lllcccccc} 
Tank \# & Core \# & Matrix & Segment \# & Date & Jar/Vial \# & Vial Size (mLs) & Net Wt\# Found (gms) & Laboratory \# \\
\hline AN-106 & Grab & GL & 2 & $8 / 27 / 97$ & 13224 & 40 & 46.1 & S96T000132 \\
AN-106 & Grab & GL & 3 & $8 / 27 / 97$ & 13225 & 40 & S96T000133 \\
AN-106 & Grab & GL & 1 & $8 / 27 / 97$ & 13226 & 40 & 46.3 & n/a \\
AN-106 & Grab & GL & 1 & $8 / 27 / 97$ & 13286 & 40 & S97T000131 \\
AN-106 & Grab & GL & 2 & $8 / 27 / 97$ & 13227 & 40 & 46.7 & n/a \\
AN-106 & Grab & GL & 5 & $8 / 27 / 97$ & 13287 & 40 & 45.9 & S97T000149 \\
AN-106 & Grab & GL & 6 & $8 / 28 / 97$ & 13229 & 40 & 48.1 & S96T000150 \\
AN-106 & Grab & GL & 7 & $8 / 28 / 97$ & 13228 & 40 & S8.5 & S96T000151 \\
& & & & & & & & Total Weight
\end{tabular}

\begin{tabular}{lllccccrc} 
Tank \# & Core \# & Matrix & Segment \# & Date & Jar/Vial \# & Vial Size (mLs) & Net Wt\# Found (gms) & Laboratory \# \\
\hline AN-107 & Grab & GL & $95-1$ & $2 / 29 / 96$ & 9281 & 60 & 76.3 & S96T000727 \\
AN-107 & Grab & GL & $95-10$ & $3 / 12 / 96$ & 9534 & 60 & 58.4 & S96T000706
\end{tabular}


Vials in 11A1 and 11A2

\begin{tabular}{|c|c|c|c|c|c|c|c|c|}
\hline Tank \# & Core \# & Matrix & Segment \# & Date & Jar/Vial \# & Vial Size (mLs) & Net Wt\# Found (gms) & Laboratory \# \\
\hline AN-107 & Grab & GL & $95-2$ & $3 / 12 / 96$ & 9279 & 60 & 57.3 & S96T000728 \\
\hline AN-107 & Grab & GL & $95-4$ & $3 / 1 / 96$ & 9349 & 60 & 67.3 & S96T000740 \\
\hline AN-107 & Grab & $\mathrm{GL}$ & $95-4$ & $3 / 1 / 96$ & 9350 & 60 & 29.7 & S96T000742 \\
\hline AN-107 & Grab & GL & $95-4$ & $3 / 12 / 96$ & 8445 & 40 & 7 & S96T001320 \\
\hline AN-107 & Grab & GL & $95-4$ & $3 / 12 / 96$ & 9280 & 60 & 69.3 & S96T000729 \\
\hline AN-107 & Grab & GL & $95-5$ & $3 / 12 / 96$ & 8446 & 40 & 15.1 & S96T001321 \\
\hline AN-107 & Grab & GL & $95-6$ & $2 / 29 / 96$ & 9282 & 60 & 81 & S96T000702 \\
\hline AN-107 & Grab & GL & $95-7$ & $2 / 29 / 96$ & 9335 & 60 & 80.3 & S96T000703 \\
\hline AN-107 & Grab & GL & $95-8$ & $2 / 29 / 96$ & 9336 & 60 & 80.4 & S96T000699 \\
\hline AN-107 & Grab & GL & $95-9$ & $3 / 12 / 96$ & 9522 & 60 & 66.8 & S96T000715 \\
\hline \multirow[t]{2}{*}{$\mathrm{AN}-107$} & Grab & GL & $95-9$ & $3 / 12 / 96$ & 9523 & 60 & 44.8 & S96T000705 \\
\hline & & & & & & Total Weight & 733.7 & \\
\hline \multirow[t]{2}{*}{ AN-107 } & Grab & Sludge & $95-10$ & $3 / 12 / 96$ & 9533 & 60 & 25.7 & S96T000716 \\
\hline & & & & & & Total Weight & 25.7 & \\
\hline Tank \# & Core \# & Matrix & Segment \# & Date & Jar/Vial \# & Vial Size (mLs) & Net Wt\# Found (gms) & Laboratory \# \\
\hline AP-101 & Grab & Gl & $95-1$ & $3 / 1 / 96$ & 9340 & 60 & 80.6 & S96T000731 \\
\hline $\mathrm{AP}-101$ & Grab & GI & $95-2$ & $3 / 1 / 96$ & 9341 & 60 & 76.2 & S96T000883 \\
\hline AP-101 & Grab & Gl & $95-3$ & $3 / 1 / 96$ & 9342 & 60 & 77.4 & S96T000884 \\
\hline AP-101 & Grab & Gl & $95-4$ & $3 / 1 / 96$ & 9343 & 60 & 81.3 & S96T000735 \\
\hline AP-101 & Grab & Gl & $95-5$ & $3 / 1 / 96$ & 9344 & 60 & 79.6 & S96T000736 \\
\hline \multirow[t]{2}{*}{$\mathrm{AP}-101$} & Grab & Gl & $95-6$ & $3 / 1 / 96$ & 9345 & 60 & 74.7 & \$96T000737 \\
\hline & & & & & & Total Weight & 469.8 & \\
\hline
\end{tabular}

\begin{tabular}{lllcccccc} 
Tank \# & Core \# & Matrix & Segment \# & Date & Jar/Vial \# & Vial Size (mLs) & Net Wt\# Found (gms) & Laboratory \# \\
\hline AP-104 & Grab & GL & $96-3 B$ & & 4AP963B & 125 & 70 & 70.7 \\
AP-104 & Grab & GL & $97-1 B$ & & 4AP971B & 125 & Total Weight & $\frac{140.7}{}$
\end{tabular}

\begin{tabular}{ccccccccc} 
Tank \# & Core \# & Matrix & Segment \# & Date & Jar/Vial \# & Vial Size (mLs) & Net Wt Found (gms) & Laboratory \# \\
\hline AP-105 & 208 & DL & 1 & $7 / 8 / 97$ & 12901 & 250 & 268.6 & S97T001719
\end{tabular}


Vials in 11A1 and 11A2

\begin{tabular}{|c|c|c|c|c|c|c|c|c|}
\hline Tank \# & Core \# & Matrix & Segment \# & Date & Jar/Vial \# & Vial Size (mLs) & Net Wt\# Found (gms) & Laboratory \# \\
\hline $\begin{array}{l}\text { AP-105 } \\
\text { AP-105 }\end{array}$ & $\begin{array}{l}208 \\
208\end{array}$ & $\begin{array}{l}\text { DL } \\
\text { DL }\end{array}$ & $\begin{array}{l}1 \\
2\end{array}$ & $\begin{array}{r}7 / 11 / 97 \\
7 / 8 / 97\end{array}$ & $\begin{array}{l}12254 \\
13051\end{array}$ & $\begin{array}{c}40 \\
60 \\
\text { Total Weight }\end{array}$ & $\begin{array}{c}50.8 \\
67.8 \\
387.2\end{array}$ & $\begin{array}{l}\text { S97T001720 } \\
\text { S97T001722 }\end{array}$ \\
\hline $\begin{array}{l}\text { AP-105 } \\
\text { AP-105 } \\
\text { AP-105 }\end{array}$ & $\begin{array}{l}208 \\
208 \\
208\end{array}$ & $\begin{array}{l}\text { Salt } \\
\text { Salt } \\
\text { Salt }\end{array}$ & $\begin{array}{l}2 \\
2 \\
2\end{array}$ & $\begin{array}{r}7 / 8 / 97 \\
7 / 11 / 97 \\
7 / 11 / 97\end{array}$ & $\begin{array}{l}11907 \\
13053 \\
13059\end{array}$ & $\begin{array}{c}125 \\
60 \\
60 \\
\text { Total Weight }\end{array}$ & $\begin{array}{c}44.1 \\
107.1 \\
87.5 \\
238.7\end{array}$ & $\begin{array}{l}\text { S97T001729 } \\
\text { S97T001731 } \\
\text { S97T001725 }\end{array}$ \\
\hline $\begin{array}{l}\mathrm{AP}-105 \\
\mathrm{AP}-105\end{array}$ & $\begin{array}{l}209 \\
209\end{array}$ & $\begin{array}{l}\text { DL } \\
\text { DL }\end{array}$ & $\begin{array}{l}1 \\
1\end{array}$ & $\begin{array}{l}7 / 15 / 97 \\
7 / 22 / 97\end{array}$ & $\begin{array}{l}12597 \\
13288\end{array}$ & $\begin{array}{c}250 \\
40 \\
\text { Total Weight }\end{array}$ & $\begin{array}{c}148.1 \\
53.4 \\
201.5\end{array}$ & $\begin{array}{l}\text { S97T001759 } \\
\text { S97T001763 }\end{array}$ \\
\hline $\begin{array}{l}\text { AP-105 } \\
\text { AP-105 } \\
\text { AP-105 } \\
\text { AP-105 } \\
\text { AP-105 } \\
\text { AP-105 } \\
\text { AP-105 }\end{array}$ & $\begin{array}{l}209 \\
209 \\
209 \\
209 \\
209 \\
209 \\
209\end{array}$ & $\begin{array}{l}\text { Salt } \\
\text { Salt } \\
\text { Salt } \\
\text { Salt } \\
\text { Salt } \\
\text { Salt } \\
\text { Salt }\end{array}$ & $\begin{array}{l}2 \\
2 \\
2 \\
3 \\
3 \\
3 \\
3\end{array}$ & $\begin{array}{l}7 / 15 / 97 \\
7 / 21 / 97 \\
7 / 21 / 97 \\
7 / 16 / 97 \\
7 / 16 / 97 \\
7 / 22 / 97 \\
7 / 22 / 97\end{array}$ & $\begin{array}{l}12598 \\
13060 \\
13062 \\
12716 \\
12896 \\
13063 \\
13069\end{array}$ & $\begin{array}{c}250 \\
60 \\
60 \\
250 \\
250 \\
60 \\
60 \\
\text { Total Weight }\end{array}$ & $\begin{array}{c}149.7 \\
88.3 \\
103.5 \\
137 \\
43.5 \\
104.8 \\
106.3 \\
733.1\end{array}$ & $\begin{array}{l}\text { S97T001767 } \\
\text { S97T001773 } \\
\text { S97T001772 } \\
\text { S07T001769 } \\
\text { S97T001768 } \\
\text { S97T001775 } \\
\text { S97T001774 }\end{array}$ \\
\hline $\begin{array}{l}\text { AP-105 } \\
\text { AP-105 }\end{array}$ & $\begin{array}{l}210 \\
210\end{array}$ & $\begin{array}{l}\text { DL } \\
\text { DL }\end{array}$ & $\begin{array}{l}2 \\
2\end{array}$ & $\begin{array}{l}7 / 16 / 97 \\
7 / 23 / 97\end{array}$ & $\begin{array}{l}12600 \\
12253\end{array}$ & $\begin{array}{c}250 \\
40 \\
\text { Total Weight }\end{array}$ & $\begin{array}{r}52.8 \\
56.7 \\
109.5\end{array}$ & $\begin{array}{l}\text { S97T001760 } \\
\text { S97T001764 }\end{array}$ \\
\hline $\begin{array}{l}\mathrm{AP}-105 \\
\mathrm{AP}-105 \\
\mathrm{AP}-105 \\
\mathrm{AP}-105\end{array}$ & $\begin{array}{l}210 \\
210 \\
210 \\
210\end{array}$ & $\begin{array}{l}\text { Salt } \\
\text { Salt } \\
\text { Salt } \\
\text { Salt }\end{array}$ & $\begin{array}{l}1 \\
1 \\
2 \\
2\end{array}$ & $\begin{array}{l}7 / 16 / 97 \\
7 / 23 / 97 \\
7 / 16 / 97 \\
7 / 23 / 97\end{array}$ & $\begin{array}{l}12706 \\
13070 \\
12898 \\
13071\end{array}$ & $\begin{array}{c}250 \\
60 \\
250 \\
60 \\
\text { Total Weight }\end{array}$ & $\begin{array}{c}191.3 \\
93 \\
122.3 \\
97.1 \\
503.7\end{array}$ & $\begin{array}{l}\text { S97T001770 } \\
\text { S97T001776 } \\
\text { S97T001771 } \\
\text { S97T001777 }\end{array}$ \\
\hline $\begin{array}{l}\text { AP-105 } \\
\text { AP-105 } \\
\text { AP-105 }\end{array}$ & $\begin{array}{l}\text { Grab } \\
\text { Grab } \\
\text { Grab }\end{array}$ & $\begin{array}{l}\text { GL } \\
\text { GL } \\
\text { GL }\end{array}$ & $\begin{array}{l}96-1 D \\
96-2 D \\
96-3 D\end{array}$ & & $\begin{array}{l}\text { 5AP961D } \\
\text { 5AP962D } \\
\text { 5AP963D }\end{array}$ & $\begin{array}{c}125 \\
125 \\
125 \\
\text { Total Weight }\end{array}$ & $\begin{array}{c}71.8 \\
94.7 \\
90.2 \\
256.7\end{array}$ & . \\
\hline
\end{tabular}


Vials in 11A1 and 11A2

\begin{tabular}{lllcccccc} 
Tank \# & Core \# & Matrix & Segment \# & Date & Jar/Vial \# & Vial Size (mLs) & Net Wt\# Found (gms) & Laboratory \# \\
\hline AP-106 & Grab & GL & 1 & $9 / 5 / 95$ & 13256 & 40 & 42.4 & S96T005178 \\
AP-106 & Grab & GL & 2 & $9 / 5 / 97$ & 13258 & 40 & 43.9 & S96T005180 \\
AP-106 & Grab & GL & 3 & $9 / 5 / 97$ & 13257 & 40 & Not.2 & NA \\
& & & & & & & &
\end{tabular}

\begin{tabular}{lllcccccc} 
Tank \# & Core \# & Matrix & Segment \# & Date & Jar/Vial \# & Vial Size (mLs) & Net Wt\# Found (gms) & Laboratory \# \\
\hline AP-108 & Grab & GL & 1 & $4 / 19 / 96$ & 9721 & 60 & 64.3 & S96T000094 \\
AP-108 & Grab & GL & 2 & $4 / 19 / 96$ & 9720 & 60 & Total Weight & S1.3
\end{tabular}

\begin{tabular}{ccccccccc} 
Tank \# & Core \# & Matrix & Segment \# & Date & Jar/Vial \# & Vial Size (mLs) & Net Wt\# Found (gms) & Laboratory \# \\
\hline AW-015 & 196 & Sludge & 8 & $5 / 27 / 97$ & 12557 & Total Weight & $\frac{62.5}{62.5}$ & S97T001139
\end{tabular}

\begin{tabular}{|c|c|c|c|c|c|c|c|c|}
\hline Tank \# & Core \# & Matrix & Segment \# & Date & Jar/Vial \# & Vial Size (mLs) & Net Wt\# Found (gms) & Laboratory \# \\
\hline AW-101 & 132 & $\mathrm{DL}$ & 2 & $3 / 5 / 96$ & 8435 & 40 & 34 & S96T000804 \\
\hline AW-101 & 132 & $\mathrm{DL}$ & 3 & $3 / 5 / 96$ & 9527 & 60 & 82.1 & S96T000819 \\
\hline AW-101 & 132 & $\mathrm{DL}$ & 4 & $3 / 5 / 96$ & 8437 & 40 & 60.5 & S96T000828 \\
\hline AW-101 & 132 & DL & 5 & $3 / 5 / 96$ & 8439 & 40 & 54.5 & S96T000842 \\
\hline \multirow[t]{2}{*}{ AW-101 } & 132 & DL & 9 & $4 / 2 / 96$ & 8631 & 125 & 34.3 & S96T001745 \\
\hline & & & & & & Total Weight & 265.4 & \\
\hline AW-101 & 132 & FB & Fblk & $4 / 2 / 96$ & 9474 & 250 & 152.8 & S96T001703 \\
\hline \multirow[t]{2}{*}{ AW-101 } & 132 & FB & Fblk & $4 / 26 / 96$ & 9770 & 40 & 40.5 & S96T001704 \\
\hline & & & & & & Total Weight & 193.3 & \\
\hline AW-101 & 132 & LL & 7 & $4 / 2 / 96$ & 8650 & 125 & 90.6 & S96T001710 \\
\hline \multirow[t]{2}{*}{ AW-101 } & 132 & LL & 7 & $4 / 26 / 96$ & 8453 & 40 & 56.5 & S96T001713 \\
\hline & & & & & & Total Weight & 147.1 & \\
\hline AW-101 & 132 & Salt & 10 & $4 / 2 / 96$ & 9432 & 125 & 16.5 & \$96T001753 \\
\hline AW-101 & 132 & Salt & 11 & $4 / 2 / 96$ & 8618 & 125 & 21.9 & S96T001769 \\
\hline AW-101 & 132 & Salt & 12 & $9 / 24 / 97$ & 13443 & 60 & 10.2 & S96T001924 \\
\hline
\end{tabular}


Vials in 11A1 and 11A2

\begin{tabular}{|c|c|c|c|c|c|c|c|c|}
\hline Tank \# & Core \# & Matrix & Segment \# & Date & Jar/Vial \# & Vial Size (mLs) & Net Wt\# Found (gms) & Laboratory \# \\
\hline AW-101 & 132 & Salt & 13 & $4 / 29 / 96$ & 9445 & 125 & 14.9 & S96T002029 \\
\hline AW-101 & 132 & Salt & 14 & $4 / 9 / 96$ & 9430 & 125 & 31.9 & \$96T001927 \\
\hline AW-101 & 132 & Salt & 15 & $4 / 29 / 96$ & 9438 & 125 & 22.5 & S96T001929 \\
\hline AW-101 & 132 & Salt & 16 & $4 / 9 / 96$ & 9446 & 125 & 24.1 & S96T001932 \\
\hline AW-101 & 132 & Salt & 3 & $3 / 5 / 96$ & 8436 & 40 & 21.9 & S $96 \mathrm{~T} 000823$ \\
\hline AW-101 & 132 & Salt & 4 & $3 / 5 / 96$ & 8438 & 40 & 20 & S $96 \mathrm{~T} 000832$ \\
\hline AW-101 & 132 & Salt & 5 & $3 / 5 / 96$ & 8440 & 40 & 20 & S96T000847 \\
\hline AW-101 & 132 & Salt & 7 & $4 / 26 / 96$ & 9814 & 60 & 94.6 & S96T001718 \\
\hline AW-101 & 132 & Salt & 7 & $9 / 29 / 97$ & 13490 & 60 & 12.1 & S96T002150 \\
\hline AW-101 & 132 & Salt & 9 & 9/29/97 & 13487 & 60 & 14.3 & na \\
\hline AW-101 & 132 & Salt & Comp & $5 / 13 / 96$ & 10251 & 250 & 46.7 & S96T002729 \\
\hline AW-101 & 132 & Salt & Comp & $6 / 26 / 96$ & 10471 & 60 & 33.1 & S96T002745 \\
\hline AW-101 & 132 & Salt & Comp & $6 / 27 / 96$ & 10473 & 60 & 47.4 & S96T002763 \\
\hline \multirow[t]{2}{*}{$\mathrm{AW}-101$} & 132 & Salt & Comp & $6 / 27 / 96$ & 10474 & 60 & 79.7 & S96T002746 \\
\hline & & & & $\sim$ & & Total Weight & 531.8 & \\
\hline AW-101 & 132 & Sludge & 1 & $2 / 22 / 96$ & 9194 & 250 & 75.7 & S96T000798 \\
\hline $\mathrm{AW}-101$ & 132 & Sludge & 1 & $2 / 22 / 96$ & 9270 & 60 & 31.5 & S96T000793 \\
\hline AW-101 & 132 & Sludge & 1 & $3 / 5 / 96$ & 9526 & 60 & 85.9 & S96T000799 \\
\hline$A W-101$ & 132 & Sludge & 18 & $4 / 9 / 96$ & 9487 & 250 & 168.5 & S96T001934 \\
\hline AW-101 & 132 & Sludge & 18 & $4 / 9 / 96$ & 9499 & 250 & 173.2 & S96T001933 \\
\hline$A W-101$ & 132 & Sludge & 2 & $2 / 23 / 96$ & 9397 & 250 & 52.2 & S96T000813 \\
\hline $\mathrm{AW}-101$ & 132 & Sludge & 2 & $3 / 5 / 96$ & 9528 & 60 & 80.5 & S96T000814 \\
\hline AW-101 & 132 & Sludge & 2 & $3 / 5 / 96$ & 9529 & 60 & 32.3 & S96T000808 \\
\hline$A W-101$ & 132 & Sludge & 20 & $4 / 9 / 96$ & 9442 & 125 & 108.7 & \$96T001935 \\
\hline AW-101 & 132 & Sludge & 20 & $4 / 9 / 96$ & 9490 & 250 & 160 & S96T001937 \\
\hline $\mathrm{AW}-101$ & 132 & Sludge & 22 & $4 / 8 / 96$ & 9498 & 250 & 154.8 & S96T001938 \\
\hline $\mathrm{AW}-101$ & 132 & Sludge & 22 & $4 / 9 / 96$ & 9443 & 125 & 84.5 & S96T001940 \\
\hline$A W-101$ & 132 & Sludge & Comp & $6 / 26 / 96$ & 10403 & 125 & 60.8 & S96T002747 \\
\hline \multirow[t]{2}{*}{$\mathrm{AW}-101$} & 132 & Sludge & Comp & $6 / 26 / 96$ & 10472 & 60 & 25.1 & S96T002748 \\
\hline & & & & & & Total Weight & 1293.7 & \\
\hline AW-101 & 139 & DL & 11 & $5 / 8 / 96$ & 10036 & 250 & 160.3 & S96T002677 \\
\hline AW-101 & 139 & DL & 11 & $6 / 27 / 96$ & 9926 & 40 & 59 & S96T003216 \\
\hline AW-101 & 139 & $\overline{D L}$ & 12 & $5 / 8 / 96$ & 10037 & 250 & 165.5 & S96T002685 \\
\hline
\end{tabular}


Vials in 11A1 and 11A2

\begin{tabular}{|c|c|c|c|c|c|c|c|c|}
\hline Tank \# & Core \# & Matrix & Segment \# & Date & Jar/Vial \# & Vial Size (mLs) & Net Wt\# Found (gms) & Laboratory \# \\
\hline $\mathrm{AW}-101$ & 139 & $\mathrm{DL}$ & 12 & $6 / 27 / 96$ & 9927 & 40 & 60.9 & S96T003218 \\
\hline$A W-101$ & 139 & $\mathrm{DL}$ & 13 & $5 / 8 / 96$ & 10038 & 250 & 160.1 & S96T002693 \\
\hline AW-101 & 139 & $\mathrm{DL}$ & 13 & $6 / 27 / 96$ & 9928 & 40 & 60.5 & S96T003220 \\
\hline AW-101 & 139 & DL & 14 & $5 / 8 / 96$ & 10040 & 250 & 189.4 & S96T002702 \\
\hline AW-101 & 139 & DL & 14 & $6 / 27 / 96$ & 9929 & 40 & 60 & S96T003222 \\
\hline AW-101 & 139 & DL & 15 & $5 / 8 / 96$ & 10041 & 250 & 166.4 & S96T002710 \\
\hline AW-101 & 139 & DL & 15 & $6 / 27 / 96$ & 9930 & 40 & 61.9 & S96T003224 \\
\hline$A W-101$ & 139 & DL & $17 \mathrm{R}$ & $5 / 29 / 96$ & 10071 & 125 & 137.2 & S96T003543 \\
\hline \multirow[t]{2}{*}{$A W-101$} & 139 & DL & 2 & $5 / 3 / 96$ & 9998 & 250 & 98.2 & S96T002525 \\
\hline & & & & & & Total Weight & 1379.4 & \\
\hline \multirow[t]{2}{*}{$A W-101$} & 139 & LL & 17 & $5 / 29 / 96$ & 10075 & 125 & 92.1 & \\
\hline & & & & & & Total Weight & 92.1 & \\
\hline AW-101 & 139 & Sait & 10 & $7 / 10 / 96$ & 9883 & 60 & 16.8 & S96T002463 \\
\hline$A W-101$ & 139 & Salt & 11 & $5 / 8 / 96$ & 10031 & 125 & 16 & S96T002680 \\
\hline AW-101 & 139 & Salt & 12 & $5 / 8 / 96$ & 10034 & 125 & 11.9 & S96T002688 \\
\hline AW-101 & 139 & Salt & 13 & $5 / 8 / 96$ & 9914 & 60 & 14.5 & S96T002696 \\
\hline $\mathrm{AW}-101$ & 139 & Salt & 14 & $5 / 8 / 96$ & 9915 & 60 & 27.2 & S96T002705 \\
\hline AW-101 & 139 & Salt & 15 & $5 / 8 / 96$ & 9916 & 60 & 25.5 & S95T002713 \\
\hline AW-101 & 139 & Salt & 16 & $5 / 28 / 96$ & 10207 & 60 & 5.6 & S96T003518 \\
\hline AW-101 & 139 & Salt & 16 & 7/9/96 & 10208 & 60 & 8.6 & S96T003237 \\
\hline AW-101 & 139 & Salt & $16 \mathrm{R}$ & $7 / 9 / 96^{\circ}$ & 10195 & 250 & 325.9 & S96T003233 \\
\hline AW-101 & 139 & Salt & $16 \mathrm{R}$ & 7/9/96 & 10529 & 60 & 25.4 & S96T003234 \\
\hline AW-101 & 139 & Salt & 16R2 & $5 / 28 / 96$ & 10210 & 60 & 24.9 & S96T003533 \\
\hline AW-101 & 139 & Salt & $17 \mathrm{R}$ & $7 / 12 / 96$ & 10530 & 60 & 29.8 & S96T003547 \\
\hline AW-101 & 139 & Salt & $17 \mathrm{R}$ & $7 / 12 / 96$ & 10532 & 60 & 28 & S96T003554 \\
\hline AW-101 & 139 & Salt & 19 & $5 / 29 / 96$ & 10218 & 250 & 30.6 & S96T003568 \\
\hline AW-101 & 139 & Salt & 19 & $7 / 12 / 96$ & 10534 & 60 & 32.5 & \$96T003570 \\
\hline AW-101 & 139 & Salt & 19 & $7 / 15 / 96$ & 10536 & 60 & 28.6 & S96T003563 \\
\hline AW-101 & 139 & Salt & 2 & $5 / 3 / 96$ & 9885 & 60 & 23.8 & S96T002528 \\
\hline AW-101 & 139 & Salt & 2 & $5 / 3 / 96$ & 9887 & 60 & 29.2 & S95T002532 \\
\hline AW-101 & 139 & Salt & 21 & $7 / 15 / 96$ & 10540 & 60 & 34.3 & S96T003577 \\
\hline AW-101 & 139 & Salt & 3 & $5 / 3 / 96$ & 9886 & 60 & 13.3 & S96T002559 \\
\hline AW-101 & 139 & Salt & 4 & $5 / 3 / 96$ & 9888 & 60 & 18.1 & S96T002576 \\
\hline
\end{tabular}


Vials in 11A1 and 11A2

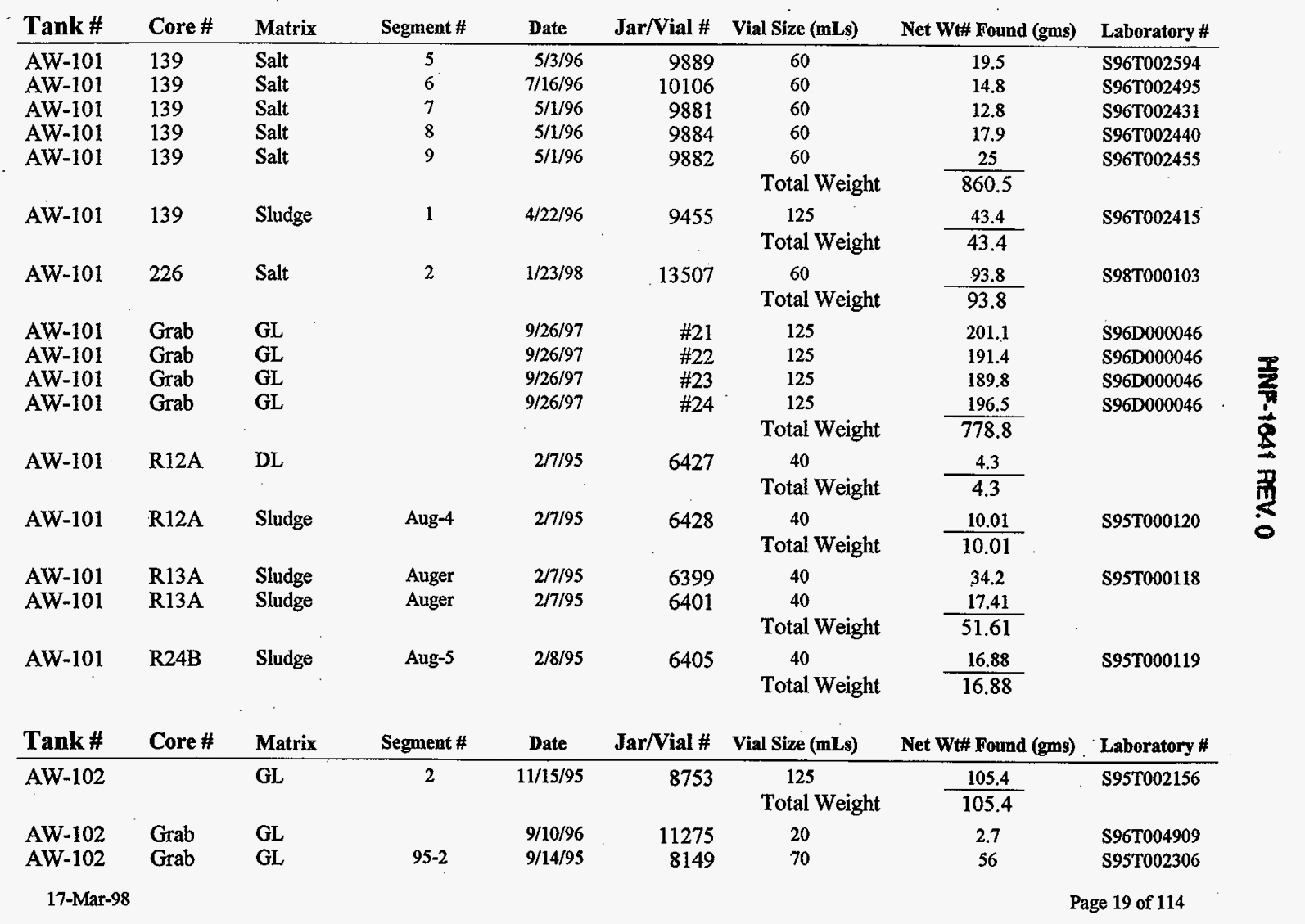


Vials in 11A1 and 11A2

\begin{tabular}{lllcccccc} 
Tank \# & Core \# & Matrix & Segment \# & Date & Jar/Vial \# & Vial Size (mLs) & Net Wt\# Found (gms) & Laboratory \# \\
\hline AW-102 & Grab & GL & $95-3$ & $9 / 14 / 95$ & 8150 & 70 & 62.7 & S95T002307 \\
AW-102 & Grab & GL & $97-1$ & & $2 A W 97-1$ & 125 & 79.6 \\
AW-102 & Grab & GL & $97-2$ & & $2 A W 97-2$ & 125 & 69 \\
& & & & & & Total Weight & 270
\end{tabular}

\begin{tabular}{|c|c|c|c|c|c|c|c|c|}
\hline Tank\# & Core \# & Matrix & Segment \# & Date & Jar/Vial \# & Vial Size (mLs) & Net Wt\# Found (gms) & Laboratory \# \\
\hline AW-103 & 193 & $\mathrm{DL}$ & 4 & $5 / 9 / 97$ & 12612 & 250 & 123 & S97T000874 \\
\hline AW-103 & 193 & DL & 4 & $6 / 5 / 97$ & 12241 & $\begin{array}{c}40 \\
\text { Total Weight }\end{array}$ & $\frac{43.4}{166.4}$ & S97T000886 \\
\hline AW-103 & 193 & FB & Fblk & $5 / 8 / 97$ & 12604 & 250 & 135.1 & S97T000877 \\
\hline AW-103 & 193 & FB & Fblk & $6 / 5 / 97$ & 12240 & $\begin{array}{c}40 \\
\text { Total Weight }\end{array}$ & $\frac{41.3}{176.4}$ & S97T000887 \\
\hline AW-103 & 193 & Sludge & & $5 / 13 / 97$ & 12602 & 250 & 12.7 & S97T000973 \\
\hline AW-103 & 193 & Sludge & 10 & $5 / 28 / 97$ & 12347 & 60 & 45 & S97T000993 \\
\hline AW-103 & 193 & Sludge & 4 & $6 / 5 / 97$ & 12357 & 40 & 43.4 & S97T000886 \\
\hline AW-103 & 193 & Sludge & 4 & $6 / 5 / 97$ & 12688 & 60 & 41.3 & S97T000883 \\
\hline AW-103 & 193 & Sludge & 5 & $5 / 9 / 97$ & 12613 & 250 & 58.1 & S97T000875 \\
\hline AW-103 & 193 & Sludge & 5 & $5 / 9 / 97$ & 12616 & 250 & 49 & S97T000876 \\
\hline AW-103 & 193 & Sludge & 5 & $6 / 5 / 97$ & 12689 & 60 & 95.9 & S97T000884 \\
\hline AW-103 & 193 & Sludge & 5 & $6 / 5 / 97$ & 12692 & 60 & 74.1 & S97T000885 \\
\hline AW-103 & 193 & Sludge & 6 & $5 / 13 / 97$ & 12609 & 250 & 79.8 & S97T000967 \\
\hline AW-103 & 193 & Sludge & 6 & $5 / 13 / 97$ & 12611 & 250 & 78.8 & S97T000971 \\
\hline AW-103 & 193 & Sludge & 6 & $5 / 28 / 97$ & 12643 & 60 & 84.6 & S97T000985 \\
\hline AW-103 & 193 & Sludge & 6 & $5 / 28 / 97$ & 12644 & 60 & 85.2 & S97T000989 \\
\hline AW-103 & 193 & Sludge & 7 & $5 / 13 / 97$ & 12293 & 250 & 93.3 & S97T000968 \\
\hline AW-103 & 193 & Sludge & 7 & $5 / 19 / 97$ & 12282 & 250 & 31.1 & S97T000972 \\
\hline AW-103 & 193 & Sludge & 7 & $5 / 28 / 97$ & 12342 & 60 & 86.5 & S97T000968 \\
\hline AW-103 & 193 & Sludge & 7 & $5 / 28 / 97$ & 12343 & 60 & 93.4 & S97T000990 \\
\hline$A W-103$ & 193 & Sludge & 8 & $5 / 13 / 97$ & 12290 & 250 & 99.7 & S97T000969 \\
\hline AW-103 & 193 & Sludge & 8 & $5 / 28 / 97$ & 12344 & 60 & 98.6 & S97T000987 \\
\hline AW-103 & 193 & Sludge & 8 & $5 / 28 / 97$ & 12345 & 60 & 101.6 & S97T000991 \\
\hline$A W-103$ & 193 & Sludge & 9 & $5 / 14 / 97$ & 12286 & 250 & 56.1 & S97T000970 \\
\hline
\end{tabular}


Vials in 11A1 and 11A2

\begin{tabular}{|c|c|c|c|c|c|c|c|c|}
\hline Tank \# & Core \# & Matrix & Segment \# & Date & Jar/Vial \# & Vial Size (mLs) & Net Wt\# Found (gms) & Laboratory \# \\
\hline AW-103 & 193 & Sludge & 9 & $5 / 14 / 97$ & 12292 & 250 & 46.5 & S97T000974 \\
\hline AW-103 & 193 & Sludge & 9 & $5 / 28 / 97$ & 12346 & 60 & 114.5 & S97T000992 \\
\hline AW-103 & 193 & Sludge & 9 & $5 / 28 / 97$ & 12627 & $\begin{array}{c}60 \\
\text { Total Weight }\end{array}$ & $\frac{95.6}{1664.8}$ & S97T000988 \\
\hline$A W-103$ & 194 & DL & 4 & $5 / 14 / 97$ & 12287 & 250 & 174.1 & S97T001078 \\
\hline AW-103 & 194 & DL & 4 & $5 / 28 / 97$ & 12231 & $\begin{array}{l}40 \\
\text { Total Weight }\end{array}$ & $\begin{array}{c}43.7 \\
217.8\end{array}$ & S97T001080 \\
\hline$A W-103$ & 194 & Sludge & 10 & $5 / 20 / 97$ & 12298 & 250 & 166.4 & S97T001029 \\
\hline AW-103 & 194 & Sludge & 10 & $5 / 30 / 97$ & 12690 & 60 & 84.1 & S97T001053 \\
\hline AW-103 & 194 & Sludge & 4 & $5 / 14 / 97$ & 12640 & 60 & 10.5 & \$97T001018 \\
\hline AW-103 & 194 & Sludge & 5 & $5 / 19 / 97$ & 12283 & 250 & 41.3 & S97T001024 \\
\hline AW-103 & 194 & Sludge & 5 & $5 / 19 / 97$ & 12284 & 250 & 71.1 & \$97T001019 \\
\hline AW-103 & 194 & Sludge & 5 & $5 / 29 / 97$ & 12651 & 60 & 92.7 & S97T001042 \\
\hline AW-103 & 194 & Sludge & 5 & $5 / 29 / 97$ & 12652 & 60 & 91 & S97T001048 \\
\hline AW-103 & 194 & Sludge & 6 & 5/19/97 & 12285 & 250 & 73.4 & S97T001020 \\
\hline AW-103 & 194 & Sludge & 6 & $5 / 19 / 97$ & 12288 & 250 & 53.4 & S97T001025 \\
\hline AW-103 & 194 & Sludge & 6 & $5 / 29 / 97$ & 12653 & 60 & 84.9 & S97T001043 \\
\hline AW-103 & 194 & Sludge & 6 & $5 / 29 / 97$ & 12654 & 60 & 90.8 & S97T001049 \\
\hline AW-103 & 194 & Sludge & 7 & $5 / 19 / 97$ & 12291 & 250 & 75.6 & S97T001021 \\
\hline AW-103 & 194 & Sludge & 7 & $5 / 29 / 97$ & 12658 & 60 & 96.2 & S97T001044 \\
\hline$A W-103$ & 194 & Sludge & 7 & $5 / 29 / 97$ & 12659 & 60 & 88.9 & S97T001050 \\
\hline AW-103 & 194 & Sludge & 8 & $5 / 19 / 97$ & 12294 & 250 & 70.5 & S97T001027 \\
\hline AW-103 & 194 & Sludge & 8 & $5 / 19 / 97$ & 12295 & 250 & 103.2 & S97T001022 \\
\hline AW-103 & 194 & Sludge & 8 & $5 / 30 / 97$ & 12655 & 60 & 90.5 & \$97T001045 \\
\hline AW-103 & 194 & Sludge & 8 & $5 / 30 / 97$ & 12656 & 60 & 104.1 & S97T001051 \\
\hline AW-103 & 194 & Sludge & 9 & $5 / 19 / 97$ & 12296 & 250 & 99.7 & S97T001028 \\
\hline AW-103 & 194 & Sludge & 9 & $5 / 19 / 97$ & 12297 & 250 & 211 & \$97T001023 \\
\hline AW-103 & 194 & Sludge & 9 & $5 / 30 / 97$ & 12645 & 60 & 114.1 & S97T001046 \\
\hline \multirow[t]{2}{*}{$\mathrm{AW}-103$} & 194 & Sludge & 9 & $5 / 30 / 97$ & 12663 & 60 & 116 & S97T001052 \\
\hline & & & & & & Total Weight & 2029.4 & \\
\hline
\end{tabular}


Vials in 11A1 and 11A2

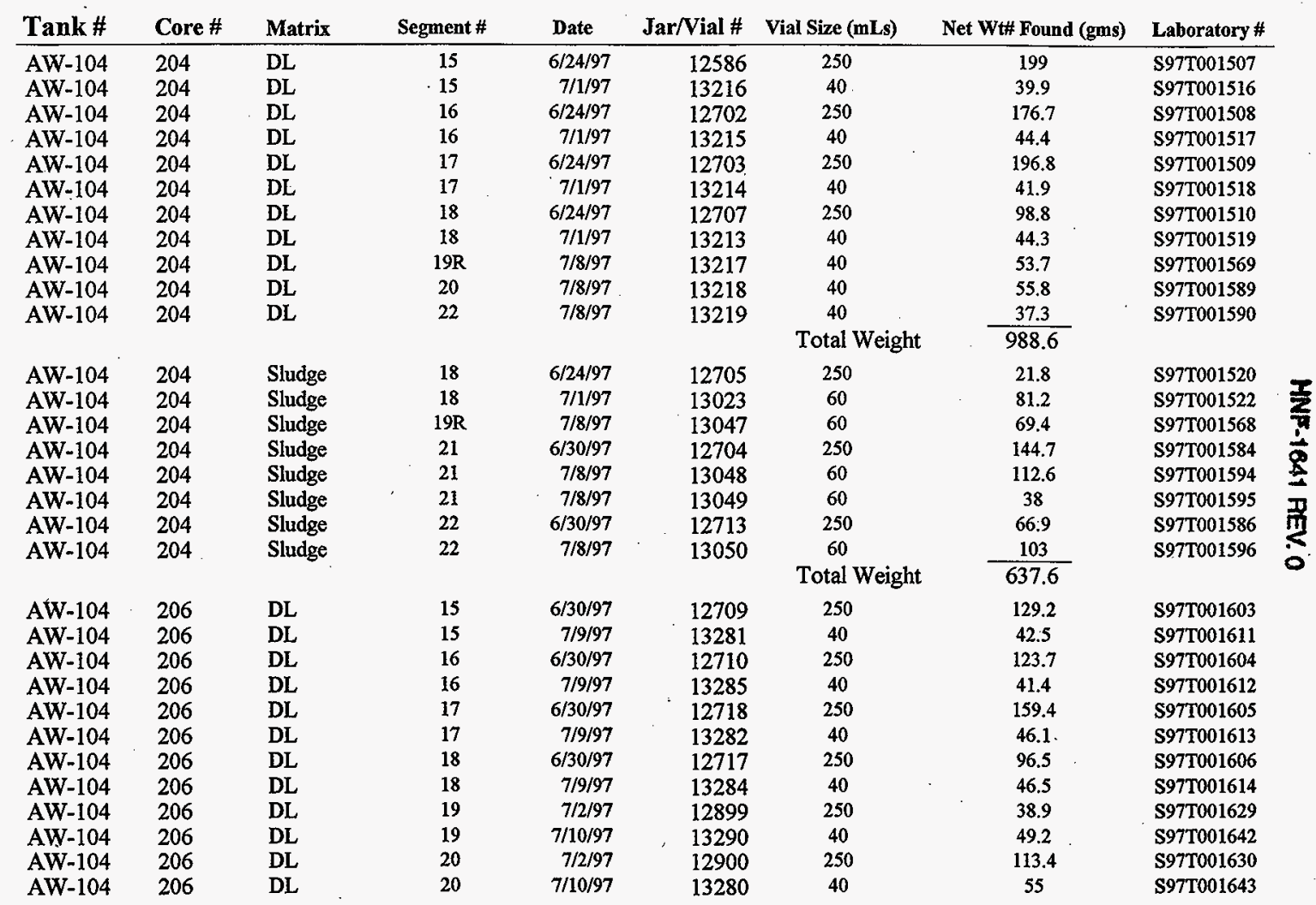


Vials in 11A1 and 11A2

\begin{tabular}{|c|c|c|c|c|c|c|c|c|}
\hline Tank \# & Core \# & Matrix & Segment \# & Date & Jar/Vial \# & Vial Size (mLs) & Net Wt\# Found (gms) & Laboratory \# \\
\hline AW-104 & 206 & DL & 21 & $7 / 2 / 97$ & 13036 & $\begin{array}{c}60 \\
\text { Total Weight }\end{array}$ & $\frac{26}{967.8}$ & S97T001644 \\
\hline AW-104 & 206 & Sludge & 18 & $6 / 30 / 97$ & 11913 & 125 & 47.1 & S97T001619 \\
\hline AW-104 & 206 & Sludge & 18 & $6 / 30 / 97$ & 11916 & 125 & 23.1 & S97T001620 \\
\hline AW-104 & 206 & Sludge & 19 & $7 / 2 / 97$ & 12370 & 125 & 21.9 & S97T001632 \\
\hline AW-104 & 206 & Sludge & 19 & $7 / 10 / 97$ & 13024 & 60 & 89.6 & S97T001652 \\
\hline AW-104 & 206 & Sludge & 19 & $7 / 10 / 97$ & 13025 & 60 & 74.4 & S97T001655 \\
\hline AW-104 & 206 & Sludge & 20 & $7 / 2 / 97$ & 11908 & 125 & 75.5 & S97T001636 \\
\hline AW-104 & 206 & Sludge & 20 & $7 / 10 / 97$ & 13026 & 60 & 50.5 & S97T001656 \\
\hline AW-104 & 206 & Sludge & 21 & $7 / 2 / 97$ & 12905 & 250 & 165.5 & S97T001633 \\
\hline AW-104 & 206 & Sludge & 21 & $7 / 10 / 97$ & 13027 & 60 & 103.3 & S97T001653 \\
\hline AW-104 & 206 & Sludge & 21 & $7 / 10 / 97$ & 13028 & 60 & 68.6 & S97T001657 \\
\hline AW-104 & 206 & Sludge & 22 & $7 / 2 / 97$ & 12595 & 250 & 176.2 & S97T001634 \\
\hline AW-104 & 206 & Sludge & 22 & $7 / 2 / 97$ & 13037 & 60 & 58.1 & S97T001658 \\
\hline AW-104 & 206 & Sludge & 22 & $7 / 11 / 97$ & 13052 & 60 & $\frac{106.7}{10605}$ & S97T001654 \\
\hline
\end{tabular}

\begin{tabular}{lllcrr} 
Tank \# & Core \# & Matrix & Segment \# & \multicolumn{1}{c}{ Date } & Jar/Vial \# \\
\hline AW-105 & 195 & DL & 4 & $5 / 20 / 97$ & 12299 \\
AW-105 & 195 & DL & 4 & $6 / 2 / 97$ & 12239 \\
& & & & & \\
AW-105 & 195 & FB & Fblk & $5 / 27 / 97$ & 12569 \\
& & & & & \\
AW-105 & 195 & Sludge & 4 & $6 / 2 / 97$ & 12348 \\
AW-105 & 195 & Sludge & 5 & $5 / 21 / 97$ & 12301 \\
AW-105 & 195 & Sludge & 5 & $5 / 21 / 97$ & 12302 \\
AW-105 & 195 & Sludge & 5 & $6 / 4 / 97$ & 12349 \\
AW-105 & 195 & Sludge & 5 & $6 / 4 / 97$ & 12350 \\
AW-105 & 195 & Sludge & 6 & $5 / 21 / 97$ & 12582 \\
AW-105 & 195 & Sludge & 6 & $6 / 3 / 97$ & 12624 \\
AW-105 & 195 & Sludge & 6 & $6 / 3 / 97$ & 12626 \\
AW-105 & 195 & Sludge & 7 & $5 / 21 / 97$ & 12300
\end{tabular}

\begin{tabular}{l} 
Vial Size (mLs) \\
\hline 250 \\
40 \\
Total Weight \\
250 \\
Total Weight \\
60 \\
250 \\
250 \\
60 \\
60 \\
250 \\
60 \\
60 \\
250
\end{tabular}

Net Wt\# Found (gms)

Laboratory \#

\$97T001100

S97T001124

S97T001166

S97T001118

S95T001089

S97T001095

S97T001113

S97T001119

S97T001096

S97T001114

S97T001120

S97T001091 
Vials in 11A1 and 11A2

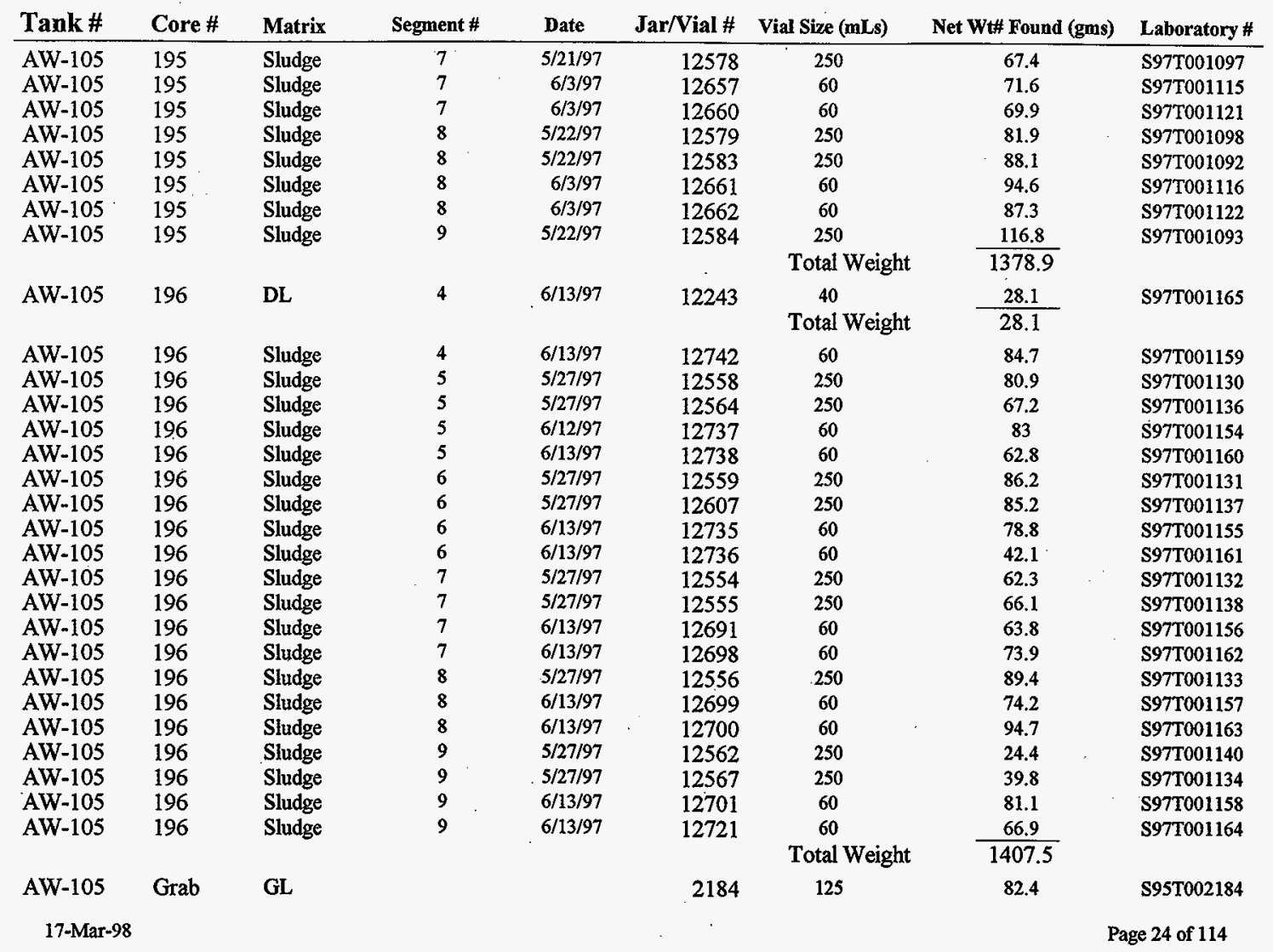


Vials in 11A1 and 11A2

\begin{tabular}{|c|c|c|c|c|c|c|c|c|}
\hline Tank \# & Core \# & Matrix & Segment \# & Date & Jar/Vial \# & Vial Size (mLs) & Net Wt\# Found (gms) & Laboratory \# \\
\hline AW-105 & Grab & GL & & & 2185 & 125 & 82.3 & S95T002185 \\
\hline AW-105 & Grab & GL & 1 & $9 / 5 / 97$ & 13247 & 40 & 42.4 & NA \\
\hline AW-105 & Grab & GL & 10 & $9 / 5 / 97$ & 13252 & 40 & 42.9 & NA \\
\hline AW-105 & Grab & GL & 11 & 9/5/97 & 13253 & 40 & 41.5 & $\mathrm{NA}$ \\
\hline AW-105 & Grab & GL & 2 & $9 / 5 / 97$ & 13249 & 40 & 41.9 & NA \\
\hline$A W-105$ & Grab & GL & 4 & $9 / 5 / 97$ & 13250 & 40 & 41.9 & NA \\
\hline AW-105 & Grab & GL & 5 & $9 / 5 / 97$ & 13251 & 40 & 44.4 & NA \\
\hline AW-105 & Grab & $\overline{G L}$ & $96-15$ & $10 / 1 / 96$ & 11389 & 60 & 50.4 & S96T005058 \\
\hline$A W-105$ & Grab & GL & $96-17$ & $10 / 1 / 96$ & 11469 & 60 & 75.1 & S96T005059 \\
\hline AW-105 & Grab & GL & $96-17$ & $10 / 1 / 96$ & 11470 & 60 & 14 & S96T005296 \\
\hline AW-105 & Grab & GL & $96-20$ & $10 / 2 / 96$ & 11472 & 60 & 72.1 & S96T005060 \\
\hline AW-105 & Grab & GL & $96-7$ & $9 / 27 / 96$ & 11384 & 60 & 9 & S96T005187 \\
\hline$A W-105$ & Grab & GL & $96-7$ & $9 / 27 / 96$ & 11385 & 60 & 74.8 & S96T005017 \\
\hline \multirow[t]{2}{*}{ AW-105 } & Grab & GL & $96-9$ & $9 / 30 / 96$ & 11387 & 60 & 61.1 & \$96T005018 \\
\hline & & & & & & Total Weight & 776.2 & \\
\hline Tank \# & Core \# & Matrix & Segment \# & Date & Jar/Vial \# & Vial Size (mLs) & Net Wt\# Found (gms) & Laboratory \# \\
\hline \multirow[t]{2}{*}{ AW-106 } & & GL & Comp. & $5 / 16 / 96$ & 9717 & 60 & 7.6 & S96V000028 \\
\hline & & & & & & Total Weight & 7.6 & \\
\hline AW-106 & Grab & GL & & & 2186 & 125 & 83.4 & S95T002186 \\
\hline AW-106 & Grab & GL & & $10 / 23 / 95$ & 7933 & 40 & 17.6 & S95T002038 \\
\hline AW-106 & Grab & GL & & $1 / 30 / 96$ & 9218 & 60 & 87.5 & S95T002015 \\
\hline AW-106 & Grab & GL & 2 & $10 / 23 / 95$ & 7856 & 70 & 88.3 & S95T002019 \\
\hline AW-106 & Grab & GL & 3 & $10 / 23 / 95$ & 7858 & 70 & 65.1 & S95T002035 \\
\hline AW-106 & Grab & GL & $97-1$ & & 6AW971 & 125 & 143.4 & \\
\hline AW-106 & Grab & GL & $97-2$ & & 6AW972 & 125 & 139.3 & \\
\hline \multirow[t]{2}{*}{ AW-106 } & Grab & GL & $97-3$ & & 6AW973 & 125 & 140.6 & \\
\hline & & & & & & Total Weight & 765.2 & \\
\hline Tank\# & Core\# & Matrix & Segment \# & Date & Jar/Vial \# & Vial Size (mLs) & Net Wt\# Found (gms) & Laboratory \# \\
\hline AW105 & 195 & FB & Fblk & $6 / 10 / 97$ & 12242 & 40 & 40.7 & \$97T001168 \\
\hline
\end{tabular}


Vials in 11A1 and 11A2

\begin{tabular}{|c|c|c|c|c|c|c|c|c|}
\hline Tank \# & Core \# & Matrix & Segment \# & Date & Jar/Vial \# & Vial Size (mLs) & Net Wt\# Found (gms) & Laboratory \# \\
\hline & & & & & & Total Weight & $\overline{40.7}$ & \\
\hline AW105 & 195 & Sludge & 9 & $6 / 4 / 97$ & 12687 & 60 & 86 & S97T001123 \\
\hline AW105 & 195 & Sludge & 9 & $6 / 5 / 97$ & 12664 & 60 & 95.5 & S97T001117 \\
\hline & & & & & & Total Weight & 181.5 & \\
\hline Tank \# & Core \# & Matrix & Segment \# & Date & Jar/Vial \# & Vial Size (mLs) & Net Wt\# Found (gms) & Laboratory \# \\
\hline$A X-101$ & 226 & DL & $11 R$ & $1 / 21 / 98$ & 12672 & 125 & 74.9 & S98T000294 \\
\hline$A X-101$ & 226 & $\mathrm{DL}$ & $11 \mathrm{R}$ & $1 / 23 / 98$ & 13883 & 40 & 25.3 & S98T000296 \\
\hline$A X-101$ & 226 & DL & 12 & $1 / 21 / 98$ & 12948 & 250 & 99.4 & \$98T000297 \\
\hline AX-101 & 226 & $\mathrm{DL}$ & 13 & $1 / 21 / 98$ & 12956 & 250 & 173.8 & S98T000298 \\
\hline$A X-101$ & 226 & DL & 13 & $1 / 23 / 98$ & 13879 & 40 & 59.7 & S98T000306 \\
\hline$A X-101$ & 226 & $\overline{D L}$ & 13 & $1 / 26 / 98$ & 13880 & 40 & 60.9 & \$98T000307 \\
\hline$A X-101$ & 226 & DL & 14 & $1 / 21 / 98$ & 12952 & 250 & 55.9 & \$98T000299 \\
\hline$A X-101$ & 226 & DL & 14 & $1 / 26 / 98$ & 13881 & 40 & 60.4 & S98T000308 \\
\hline AX-101 & 226 & DL & 15 & $2 / 19 / 98$ & 14027 & 60 & 38.3 & \\
\hline & & & & & & Total Weight & 648.6 & \\
\hline AX-101 & 226 & LL & 9 & $1 / 20 / 98$ & 13664 & 60 & 22.4 & \\
\hline & & & & & & Total Weight & 22.4 & \\
\hline$A X-101$ & 226 & Salt & 1 & $1 / 23 / 98$ & 13663 & 60 & 44.4 & S98T000086 \\
\hline AX-101 & 226 & Salt & 10 & $1 / 20 / 98$ & 12954 & 250 & 90.7 & S98T000236 \\
\hline$A X-101$ & 226 & Salt & .10 & $1 / 20 / 98$ & 12955 & 250 & 97.4 & S98T000232 \\
\hline$A X-101$ & 226 & Salt & 10 & $1 / 23 / 98$ & 13686 & 60 & 100.5 & S98T000256 \\
\hline$A X-101$ & 226 & Salt & 10 & $1 / 26 / 98$ & 13685 & 60 & 106.5 & S98T000255 \\
\hline$A X-101$ & 226 & Salt & $11 \mathrm{R}$ & $1 / 23 / 98$ & 13682 & 60 & 44.5 & S98T000258 \\
\hline AX-101 & 226 & Salt & $11 \mathrm{R}$ & $1 / 23 / 98$ & 13687 & 60 & 60.9 & S98T000257 \\
\hline AX-101 & 226 & Salt & 12 & $1 / 23 / 98$ & 13683 & 60 & 77.6 & S98T000259 \\
\hline$A X-101$ & 226 & Salt & 12 & $1 / 23 / 98$ & 13684 & 60 & 50.3 & S98T000260 \\
\hline AX-101 & 226 & Salt & 13 & $1 / 26 / 98$ & 13510 & 60 & 88.2 & S98T000261 \\
\hline AX-101 & 226 & Salt & 14 & $1 / 26 / 98$ & 13689 & 60 & 97.9 & \$98T000263 \\
\hline AX-101 & 226 & Salt & 15 & $2 / 19 / 98$ & 14028 & 60 & 29 & \\
\hline$A X-101$ & 226 & Salt & 2 & $1 / 12 / 98$ & 12936 & 250 & 89.1 & S98T000091 \\
\hline$A X-101$ & 226 & Salt & 2 & $1 / 12 / 98$ & 12937 & 250 & 68 & S98T000092 \\
\hline 17-Mar & & & & & & & & ge 26 of 114 \\
\hline
\end{tabular}


Vials in 11A1 and 11A2

\begin{tabular}{|c|c|c|c|c|c|c|c|c|}
\hline Tank \# & Core \# & Matrix & Segment \# & Date & Jar/Vial \# & Vial Size (mLs) & Net W'\# Found (gms) & Laboratory \# \\
\hline AX-101 & 226 & Salt & 2 & $1 / 23 / 98$ & 13665 & 60 & 95.7 & S98T000102 \\
\hline $\mathrm{AX}-101$ & 226 & Salt & 3 & $1 / 12 / 98$ & 12933 & 250 & 90.4 & S98T000104 \\
\hline AX-101 & 226 & Salt & 3 & $1 / 12 / 98$ & 12939 & 250 & 76.2 & S98T000101 \\
\hline AX-10I & 226 & Salt & 3 & $1 / 23 / 98$ & 13509 & 60 & 103.6 & S98T000116 \\
\hline AX-101 & 226 & Salt & 3 & $1 / 23 / 98$ & 14022 & 60 & 100.8 & S98T000115 \\
\hline AX-101 & 226 & Salt & 4 & $1 / 14 / 98$ & 12941 & 250 & 101.2 & S98T000117 \\
\hline $\mathrm{AX}-101$ & 226 & Salt & 4 & $1 / 14 / 98$ & 12944 & 250 & 96.3 & S98T000112 \\
\hline$A X-101$ & 226 & Salt & 4 & $1 / 23 / 98$ & 13671 & 60 & 99.5 & S98T000117 \\
\hline AX-101 & 226 & Salt & 4 & $1 / 23 / 98$ & 13672 & 60 & 89.5 & S98T000118 \\
\hline AX-101 & 226 & Salt & 5 & $1 / 14 / 98$ & 12934 & 250 & 94.4 & S98T000113 \\
\hline AX-101 & 226 & Salt & 5 & $1 / 14 / 98$ & 12942 & 250 & 94 & S98T000114 \\
\hline $\mathrm{AX}-101$ & 226 & Salt & 5 & $1 / 23 / 98$ & 13674 & 60 & 105.5 & S98T000119 \\
\hline AX-101 & 226 & Salt & 5 & $1 / 23 / 98$ & 13675 & 60 & 95.5 & S98T000120 \\
\hline AX-101 & 226 & Salt & 6 & $1 / 15 / 98$ & 12938 & 250 & 95.7 & \$98T000144 \\
\hline AX-101 & 226 & Salt & 6 & $1 / 15 / 98$ & 12943 & 250 & 116.3 & S98T000143 \\
\hline$A X-101$ & 226 & Salt & 6 & $1 / 23 / 98$ & 13676 & 60. & 95.5 & S98T000151 \\
\hline AX-101 & 226 & Salt & 6 & $1 / 23 / 98$ & 13677 & 60 & 97.5 & S98T000152 \\
\hline$A X-101$ & 226 & Salt & 7 & $1 / 15 / 98$ & 12935 & 250 & 95.6 & S98T000145 \\
\hline $\mathrm{AX}-101$ & 226 & Salt & 7 & $1 / 15 / 98$ & 12940 & 250 & 79.6 & S98T000146 \\
\hline $\mathrm{AX}-101$ & 226 & Salt & 7 & $1 / 23 / 98$ & 13678 & 60 & 106.1 & S98T000153 \\
\hline $\mathrm{AX}-101$ & 226 & Salt & 7 & $1 / 23 / 98$ & 13679 & 60 & 97 & S98T000154 \\
\hline $\mathrm{AX}-101$ & 226 & Salt & 9 & $1 / 20 / 98$ & 12947 & 250 & 106.4 & S98T000226 \\
\hline AX-101 & 226 & Salt & 9 & $1 / 20 / 98$ & 12949 & 250 & 88.9 & S98T000225 \\
\hline $\mathrm{AX}-101$ & 226 & Salt & 9 & $1 / 26 / 98$ & 13680 & 60 & 96.2 & S98T000231 \\
\hline \multirow[t]{2}{*}{ AX-101 } & 226 & Salt & 9 & $1 / 26 / 98$ & 13681 & 60 & 106.9 & S98T000241 \\
\hline & & & & & & Total Weight & 3469.3 & \\
\hline \multirow[t]{2}{*}{ AX-101 } & 228 & LL & 1 & 2/19/98 & 14031 & 60 & 23.6 & \\
\hline & & & & & & Total Weight & 23.6 & \\
\hline$A X-101$ & 228 & Salt & 2 & & 12968 & 250 & 186.9 & \\
\hline \multirow[t]{2}{*}{$A X-101$} & 228 & Salt & 2 & $2 / 20 / 98$ & 12962 & 250 & 173.1 & \\
\hline & & & & & & Total Weight & 360 & \\
\hline AX-101 & 266 & Salt & 14 & $1 / 26 / 98$ & 13688 & 60 & 61.4 & S98T000262 \\
\hline
\end{tabular}


Vials in 11A1 and 11A2

\begin{tabular}{lllcccccc} 
Tank \# & Core \# & Matrix & Segment \# & Date & Jar/Vial \# & Vial Size (mLs) & Net Wt\# Found (gms) & Laboratory \# \\
\hline & & & & & & Total Weight & 61.4 \\
AX-101 & Grab & GL & 1 & $9 / 4 / 97$ & 13246 & 40 & 59.2 & S97T001905 \\
AX-101 & Grab & GL & 2 & $9 / 4 / 97$ & 13248 & 40 & 59.3 & S97T001906 \\
AX-101 & Grab & GL & -3 & $9 / 4 / 97$ & 13244 & 40 & 50.1 & S97T001907 \\
AX-101 & Grab & GL & 4 & $9 / 4 / 97$ & 13245 & 40 & Total Weight & $\frac{60.6}{239.2}$
\end{tabular}

\begin{tabular}{|c|c|c|c|c|c|c|c|c|}
\hline Tank \# & Core \# & Matrix & Segment \# & Date & Jar/Vial \# & Vial Size (mLs) & Net Wt\# Found (gms) & Laboratory \# \\
\hline AX-102 & & GL & Grab & $2 / 20 / 98$ & 14038 & $\begin{array}{c}60 \\
\text { Total Weight }\end{array}$ & $\frac{36}{36}$ & S98T000480 \\
\hline$A X-102$ & Grab & Sludge & Comp & $2 / 13 / 98$ & 14114 & $\begin{array}{l}500 \\
\text { Total Weight }\end{array}$ & $\frac{110.5}{110.5}$ & S98T000481 \\
\hline AX-102 & R9E & DL & & $3 / 1 / 95$ & 6407 & $\begin{array}{l}40 \\
\text { Total Weight }\end{array}$ & $\begin{array}{l}1.92 \\
1.92\end{array}$ & ${ }^{\circ}$ \\
\hline
\end{tabular}

\begin{tabular}{|c|c|c|c|c|c|c|c|c|}
\hline Tank\# & Core \# & Matrix & Segment \# & Date & Jar/Vial \# & Vial Size (mLs) & Net Wt\# Found (gms) & Laboratory \# \\
\hline$A X-103$ & 212 & Salt & 2 & $8 / 5 / 97$ & 12817 & 250 & 27.9 & S97T001886 \\
\hline$A X-103$ & 212 & Salt & 2 & $8 / 13 / 97$ & 13087 & $\begin{array}{c}60 \\
\text { Total Weight }\end{array}$ & $\frac{109}{136.9}$ & S97T001890 \\
\hline$A X-103$ & 214 & Salt & 1 & $8 / 13 / 97$ & 11918 & 125 & 54.9 & S97T001941 \\
\hline$A X-103$ & 214 & Salt & 1 & $8 / 13 / 97$ & 13088 & 60 & 98.9 & S97T001949 \\
\hline$A X-103$ & 214 & Salt & 1 & $8 / 13 / 97$ & 13089 & 60 & 86.1 & S97T001950 \\
\hline$A X-103$ & 214 & Salt & 2 & & 12819 & 250 & 53.4 & S97T001943 \\
\hline $\mathrm{AX}-103$ & 214 & Salt & 2 & $8 / 13 / 97$ & 12818 & 250 & 66.9 & S97T001944 \\
\hline$A X-103$ & 214 & Salt & 2 & $8 / 13 / 97$ & 13090 & 60 & 96.2 & S97T001951 \\
\hline \multirow[t]{2}{*}{$A X-103$} & 214 & Salt & 2 & $8 / 13 / 97$ & 13091 & 60 & 98.4 & S97T001952 \\
\hline & & & & & & Total Weight & 554.8 & \\
\hline
\end{tabular}

\begin{tabular}{|c|c|c|c|c|c|c|c|c|}
\hline Tank \# & Core \# & Matrix & Segment \# & Date & Jar/Vial \# & Vial Size (mLs) & Net Wt\# Found (gms) & Laboratory \# \\
\hline AX-104 & & Sludge & Aug-1 & $11 / 17 / 97$ & 11848 & 125 & 92.2 & S97T002280 \\
\hline
\end{tabular}


Vials in 11A1 and 11A2

\begin{tabular}{|c|c|c|c|c|c|c|c|c|}
\hline Tank \# & Core \# & Matrix & Segment \# & Date & Jar/Vial \# & Vial Size (mLs) & Net Wt\# Found (gms) & Laboratory \# \\
\hline AX-104 & & Sludge & Aug 2 & $1 / 8 / 98$ & 14068 & 60 & 36 & S97T002281 \\
\hline $\mathrm{AX}-104$ & & Sludge & Aug-3 & $12 / 1 / 97$ & 11846 & 125 & 76.3 & S97T002282 \\
\hline AX-104 & & Sludge & Aug-4 & & 11844 & 125 & 33.5 & S97T002283 \\
\hline & & & & & & Total Weight & 238 & \\
\hline
\end{tabular}

\begin{tabular}{|c|c|c|c|c|c|c|c|c|}
\hline Tank\# & Core \# & Matrix & Segment \# & Date & Jar/Vial \# & Vial Size (mLs) & Net Wt\# Found (gms) & Laboratory \# \\
\hline AY-101 & Grab & GL & $96-1$ & $3 / 18 / 96$ & 9563 & 60 & 55.5 & S96T001267 \\
\hline$A Y-101$ & Grab & GL & $96-10$ & $3 / 29 / 96$ & 9685 & 60 & 52.1 & S96T001374 \\
\hline AY-101 & Grab & GL & $96-2$ & $3 / 18 / 96$ & 9559 & 60 & 64.3 & S96T001268 \\
\hline$A Y-101$ & Grab & GL & $96-3$ & $3 / 18 / 96$ & 9560 & 60 & 62.6 & S96T001269 \\
\hline$A Y-101$ & Grab & GL & $96-4$ & $3 / 14 / 96$ & 9542 & 60 & 26.2 & S96T001315 \\
\hline$A Y-101$ & Grab & GL & $96-5$ & $3 / 18 / 96$ & 8450 & 40 & 12.9 & S96T001316 \\
\hline AY-101 & Grab & GL & $96-5$ & $7 / 16 / 96$ & 9565 & 60 & 18.8 & S96T001274 \\
\hline$A Y-101$ & Grab & GL & $96-6$ & $3 / 18 / 96$ & 9667 & 60 & 60.2 & S96T001362 \\
\hline AY-101 & Grab & GL & $96-7$ & $3 / 18 / 96$ & 9668 & 60 & 56.8 & S96T001363 \\
\hline$A Y-101$ & Grab & GL & $96-8$ & $3 / 18 / 96$ & 9669 & 60 & 64.3 & S96T001364 \\
\hline$A Y-101$ & Grab & GL & $96-9$ & $3 / 29 / 96$ & 9684 & 60 & 38.8 & S96T001373 \\
\hline$A Y-101$ & Grab & GL & $97-1$ & & & 125 & 120.5 & S97V000001 \\
\hline$A Y-101$ & Grab & GL & $97-2 \mathrm{C}$ & & & 125 & 100.4 & S97V000004 \\
\hline AY-101 & Grab & GL & $97-2 \mathrm{E}$ & & & 125 & 51.2 & \\
\hline$A Y-101$ & Grab & $\overline{G L}$ & $97-4 \mathrm{C}$ & & $1 \mathrm{AY} 974 \mathrm{C}$ & 125 & 96.4 & S97V000020 \\
\hline \multirow{2}{*}{ AY-101 } & Grab & GL & $97-4 \mathrm{E}$ & $5 / 19 / 97$ & 12641 & 60 & 17.2 & S97V000034 \\
\hline & & & & & & Total Weight & 898.2 & \\
\hline AY-101 & Grab & GS & 4 & $3 / 13 / 96$ & 9550 & 60 & 27.7 & S96T001377 \\
\hline \multirow[t]{2}{*}{$A Y-101$} & Grab & GS & $96-4$ & $3 / 14 / 96$ & 9543 & 60 & 49.7 & S96T001273 \\
\hline & & & & & & Total Weight & 77.4 & \\
\hline
\end{tabular}

\begin{tabular}{lclcccccc} 
Tank \# & Core \# & Matrix & Segment \# & Date & Jar/Vial \# & Vial Size (mLs) & Net Wt\# Found (gms) & Laboratory \# \\
\hline AY-102 & & GL & & $11 / 9 / 95$ & 8632 & 125 & $\frac{62.3}{3}$ & S95T002913 \\
& & & & & & Total Weight & 62.3 & \\
AY-102 & Grab & GL & 11 & $8 / 29 / 97$ & 13232 & 40 & 41.5 & S96T004267
\end{tabular}


Vials in 11A1 and 11A2

\begin{tabular}{|c|c|c|c|c|c|c|c|c|}
\hline Tank \# & Core\# & Matrix & Segment \# & Date & Jar/Vial \# & Vial Size (mLs) & Net Wt\# Found (gms) & Laboratory \# \\
\hline$A Y-102$ & Grab & GL & 12 & $8 / 29 / 97$ & 13233 & 40 & 41.2 & S96T004268 \\
\hline$A Y-102$ & Grab & GL & 13 & $8 / 29 / 97$ & 13235 & 40 & 40.9 & na \\
\hline AY-102 & Grab & GL & 14 & $8 / 29 / 97$ & 13234 & 40 & 42.7 & S96T004240 \\
\hline AY-102 & Grab & GL & $97-5$ & & $2 A Y 97-5$ & 125 & 82.4 & \\
\hline$A Y-102$ & Grab & GL & $97-5$ & $1 / 21 / 98$ & 13658 & 60 & 53.3 & S98T000179 \\
\hline & & & & & & Total Weight & 302 & \\
\hline
\end{tabular}

\begin{tabular}{lllcccccc} 
Tank \# & Core \# & Matrix & Segment \# & Date & Jar/Vial \# & Vial Size (mLs) & Net Wt\# Found (gms) & Laboratory \# \\
\hline B-101 & 90 & DL & 2 & $7 / 6 / 95$ & 7403 & 40 & & 43.7 \\
\end{tabular}

$\begin{array}{ccccccccc}\text { B-101 } & 90 & \text { Salt } & 2 \mathrm{~L} / \mathrm{H} & 12 / 8 / 97 & 13449 & 60 & 8.7 & \text { S95T001554 } \\ & & & & & \text { Total Weight } & \end{array}$

$\begin{array}{lll}\text { B-101 } & 90 & \text { Sludge } \\ \text { B-101 } & 90 & \text { Sludge } \\ \text { B-101 } & 90 & \text { Sludge } \\ \text { B-101 } & 90 & \text { Sludge } \\ \text { B-101 } & 90 & \text { Sludge }\end{array}$

7/7/95

$7 / 7 / 95$

$7 / 7 / 95$

9/9/97

$9 / 12 / 97$

DL

$\begin{array}{ll}\text { B-101 } & 91 \\ & \\ \text { B-101 } & 91 \\ \text { B-101 } & 91 \\ & \\ \text { B-101 } & 91 \\ \text { B-101 } & 91 \\ \text { B-101 } & 91 \\ \text { B-101 } & 91\end{array}$

17-Mar-98

$\begin{array}{rccc}7405 & 40 & 42.6 & \text { S95T001216 } \\ 7406 & 40 & 37.9 & \text { S95T001219 } \\ 7407 & 40 & 34.4 & \text { S95T001222 } \\ 13527 & 60 & 49.8 & \text { S95T001198 } \\ 13469 & 60 & 35.4 & \text { S95T001198 } \\ & \text { Total Weight } & 200.1 & \\ 7408 & 40 & 22.9 & \text { S95T001249 } \\ & \text { Total Weight } & 22.9 & \\ 7257 & 250 & 180.9 & \\ 7402 & 40 & 40.2 & \text { S95T001234 } \\ & \text { Total Weight } & 221.1 & \\ 14024 & 60 & 18.6 & \text { S9ST001240 } \\ 13159 & 60 & 81.2 & \text { S95T001208 } \\ 7410 & 40 & 34 & \text { S95T001246 } \\ 13491 & 60 & 45.3 & \text { S95T001213 } \\ & \text { Total Weight } & 179.1 & \end{array}$


Vials in 11A1 and 11A2

\begin{tabular}{|c|c|c|c|c|c|c|c|c|}
\hline Tank \# & Core \# & Matrix & Segment \# & Date & Jar/Vial \# & Vial Size (mLs) & Net Wt\# Found (gms) & Laboratory \# \\
\hline \multirow[t]{2}{*}{ B-102 } & \multirow[t]{2}{*}{$\mathrm{RI}$} & \multirow[t]{2}{*}{ Sludge } & \multirow[t]{2}{*}{ Auger } & \multirow[t]{2}{*}{$10 / 19 / 94$} & \multirow[t]{2}{*}{7020} & \multirow{2}{*}{$\begin{array}{c}40 \\
\text { Total Weight }\end{array}$} & 3.2 & \multirow[t]{2}{*}{ S95T000195 } \\
\hline & & & & & & & 3.2 & \\
\hline Tank \# & Core \# & Matrix & Segment \# & Date & Jar/Vial \# & Vial Size (mLs) & Net Wt\# Found (gms) & Laboxatory \# \\
\hline \multirow[t]{2}{*}{ B-104 } & \multirow[t]{2}{*}{88} & \multirow[t]{2}{*}{$\overline{\mathrm{DL}}$} & \multirow[t]{2}{*}{1} & \multirow[t]{2}{*}{$1 / 24 / 96$} & \multirow[t]{2}{*}{8429} & 40 & 57 & \multirow[t]{2}{*}{ \$95T001007 } \\
\hline & & & & & & Total Weight & 57 & \\
\hline B-104 & 88 & FB & Fblk & $6 / 7 / 95$ & 7142 & 250 & 82.5 & \multirow[t]{3}{*}{ S95T001010 } \\
\hline \multirow[t]{2}{*}{ B-104 } & \multirow[t]{2}{*}{88} & \multirow[t]{2}{*}{ FB } & \multirow[t]{2}{*}{ Folk } & \multirow[t]{2}{*}{$6 / 14 / 95$} & \multirow[t]{2}{*}{7212} & 40 & 42.3 & \\
\hline & & & & & & Total Weight & 124.8 & \\
\hline B-104 & 88 & Salt & 2 & $6 / 7 / 95$ & 8026 & 40 & 35.4 & \\
\hline \multirow[t]{2}{*}{ B-104 } & \multirow[t]{2}{*}{88} & \multirow[t]{2}{*}{ Salt } & \multirow[t]{2}{*}{ Comp } & \multirow[t]{2}{*}{$9 / 9 / 97$} & \multirow[t]{2}{*}{13534} & 60 & 109.3 & \\
\hline & & & & & & Total Weight & 144.7 & \\
\hline B-104 & 88 & Sludge & 1 & $6 / 7 / 95$ & 7197 & 40 & 57.9 & \\
\hline B-104 & 88 & Sludge & 2 & $6 / 7 / 95$ & 7190 & 40 & 61.9 & \\
\hline B-104 & 88 & Sludge & 2 & $1 / 24 / 96$ & 8427 & 40 & 46.2 & S95T001052 \\
\hline B-104 & 88 & Sludge & 2 & $1 / 24 / 96$ & 8428 & 40 & 49.5 & S95T001053 \\
\hline B-104 & 88 & Sludge & 3 & $6 / 8 / 95$ & 7200 & 40 & 62.3 & \\
\hline B-104 & 88 & Sludge & 3 & $6 / 8 / 95$ & 7201 & 40 & 62.6 & \\
\hline B-104 & 88 & Sludge & 3 & $1 / 21 / 96$ & 8421 & 40 & 64.1 & \\
\hline B-104 & 88 & Sludge & 4 & $1 / 21 / 95$ & 8423 & 40 & 61 & \\
\hline B-104 & 88 & Sludge & 4 & $6 / 12 / 95$ & 7205 & 40 & 59 & \\
\hline B-104 & 88 & Sludge & 4 & $6 / 12 / 95$ & 7206 & 40 & 57.3 & \\
\hline B-104 & 88 & Sludge & 5 & $6 / 12 / 95$ & 7207 & 40 & 57.1 & \\
\hline B-104 & 88 & Sludge & 5 & $6 / 12 / 95$ & 7208 & 40 & 57.4 & \\
\hline B-104 & 88 & Sludge & 5 & $1 / 21 / 96$ & 8425 & 40 & 60.2 & \\
\hline B-104 & 88 & Sludge & 5 & $7 / 10 / 96$ & 10113 & 60 & 102.4 & S95T001074 \\
\hline B-104 & 88 & Sludge & 6 & $6 / 14 / 95$ & 7209 & 40 & 59.3 & \\
\hline B-104 & 88 & Sludge & 6 & $6 / 14 / 95$ & 7211 & 40 & 60.3 & \\
\hline B-104 & 88 & Sludge & 6 & $5 / 17 / 96$ & 10112 & 60 & 56.8 & \\
\hline B-104 & 88 & Sludge & 6 & $5 / 17 / 96$ & 10114 & 60 & 83.5 & \\
\hline B-104 & 88 & Sludge & 7 & $6 / 14 / 95$ & 7216 & 40 & 59 & \\
\hline B-104 & 88 & Sludge & 7 & $6 / 14 / 95$ & 7217 & 40 & 59.5 & \\
\hline
\end{tabular}


Vials in 11A1 and 11A2

\begin{tabular}{|c|c|c|c|c|c|c|c|c|}
\hline Tank \# & Core\# & Matrix & Segment \# & Date & Jar/Vial \# & Vial Size (mLs) & Net Wt\# Found (gms) & Laboratory \# \\
\hline B-104 & 88 & Sludge & 7 & $9 / 9 / 97$ & 13464 & 60 & 45.2 & S95T001097 \\
\hline \multirow[t]{2}{*}{ B-104 } & 88 & Sludge & 7 & $9 / 9 / 97$ & 13536 & 60 & 30.7 & S95T001098 \\
\hline & & & & & & Total Weight & 1313.2 & \\
\hline \multirow[t]{2}{*}{ B-104 } & 89 & DL & 1 & $6 / 22 / 95$ & 7218 & 40 & 54 & \\
\hline & & & & & & Total Weight & 54 & \\
\hline B-104 & 89 & Sludge & 1 & $6 / 14 / 95$ & 7243 & 250 & 42.9 & S95T001106 \\
\hline B-104 & 89 & Sludge & 1 & $6 / 22 / 95$ & 7219 & 40 & 58.3 & \\
\hline B-104 & 89 & Sludge & 1 & $6 / 22 / 95$ & 7220 & 40 & 58.5 & \\
\hline B-104 & 89 & Sludge & 1 & 9/15/97 & 11843 & 125 & 173.3 & na \\
\hline B-104 & 89 & Sludge & 2 & $6 / 22 / 95$ & 7221 & 40 & 60.6 & S95T001158 \\
\hline B-104 & 89 & Sludge & 2 & $6 / 22 / 95$ & 7222 & 40 & 52.8 & S95T001159 \\
\hline B-104 & 89 & Sludge & 2 & $1 / 17 / 96$ & 8416 & $40^{\circ}$ & 40.5 & S95T001111 \\
\hline B-104 & 89 & Sludge & 2 & $1 / 17 / 96$ & 8417 & 40 & 40.8 & S95T001112 \\
\hline B-104 & 89 & Sludge & 3 & $6 / 22 / 95$ & 7223 & 40 & 61.1 & S95T001160 \\
\hline B-104 & 89 & Sludge & 3 & $6 / 22 / 95$ & 7224 & 40 & 59.4 & \$95T001 161 \\
\hline B-104 & 89 & Sludge & 3 & $9 / 16 / 97$ & 13166 & 60 & 29.7 & S95T001128 \\
\hline B-104 & 89 & Sludge & 3 & $9 / 24 / 97$ & 13451 & 60 & 21.3 & \$95T001129 \\
\hline B-104 & 89 & Sludge & 4 & $6 / 22 / 95$ & 7225 & 40 & 60.4 & S95T001162 \\
\hline B-104 & 89 & Sludge & 4 & $6 / 22 / 95$ & 7226 & 40 & 59.9 & S95T001163 \\
\hline B-104 & 89 & Sludge & 4 & $9 / 12 / 97$ & 13470 & 60 & 50.2 & S95T001134 \\
\hline B-104 & 89 & Sludge & 4 & $9 / 15 / 97$ & 13484 & 60 & 55 & S95T001135 \\
\hline B-104 & 89 & Sludge & 5 & $6 / 20 / 95$ & 7252 & 250 & 104.7 & S95T001141 \\
\hline B-104 & 89 & Sludge & 5 & $6 / 26 / 95$ & 7227 & 40 & 60.9 & S95T001164 \\
\hline B-104 & 89 & Sludge & 5 & $6 / 26 / 95$ & 7228 & 40 & 60 & S95T001165 \\
\hline B-104 & 89 & Sludge & 5 & $9 / 15 / 97$ & 13164 & 60 & 77.9 & \$95T001140 \\
\hline B-104 & 89 & Sludge & 6 & $6 / 26 / 95$ & 7229 & 40 & 59.8 & S95T001166 \\
\hline B-104 & 89 & Sludge & 6 & $6 / 26 / 95$ & 7231 & 40 & 58.6 & S95T001167 \\
\hline B-1.04 & 89 & Sludge & 6 & $1 / 17 / 96$ & 8418 & 40 & 62.1 & S95T001146 \\
\hline B-104 & 89 & Sludge & 6 & $1 / 17 / 96$ & 8419 & 40 & 60.2 & S95T001147 \\
\hline B-104 & 89 & Sludge & 7 & $6 / 26 / 95$ & 7232 & 40 & 60.5 & S95T001168 \\
\hline B-104 & 89 & Sludge & 7 & $6 / 26 / 95$ & 7233 & 40 & 61 & S95T001169 \\
\hline B-104 & 89 & Sludge & 7 & $1 / 16 / 96$ & 9018 & 60 & 81.1 & S95T001153 \\
\hline B-104 & 89 & Sludge & 7 & $1 / 16 / 96$ & 9028 & 60 & 81.7 & S95T001152 \\
\hline
\end{tabular}


Vials in 11A1 and 11A2

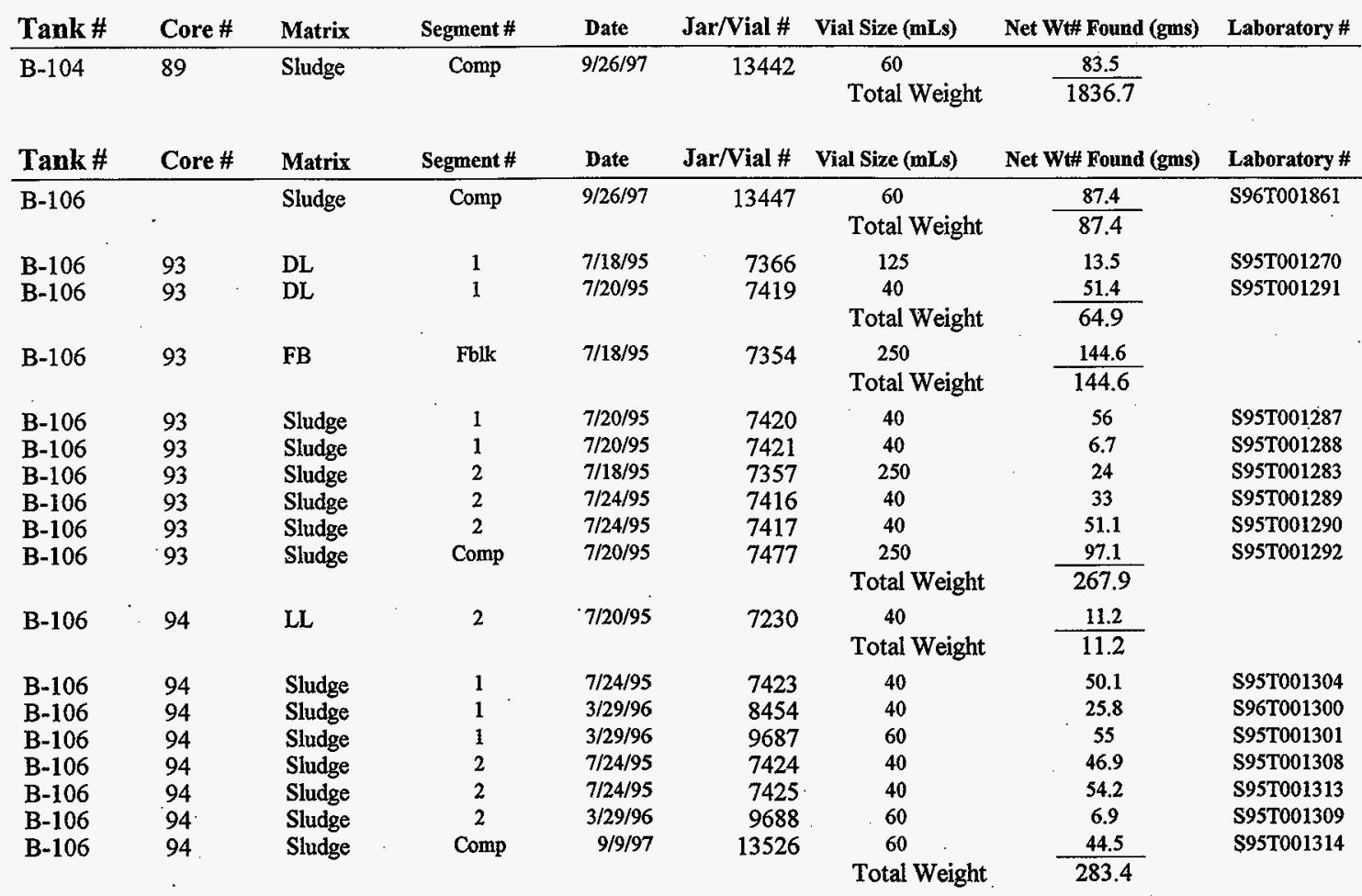


Vials in 11A1 and 11A2

\begin{tabular}{|c|c|c|c|c|c|c|c|c|}
\hline Tank \# & Core \# & Matrix & Segment \# & Date & Jar/Vial \# & Vial Size (mLs) & Net Wt\# Found (gms) & Laboratory \# \\
\hline B-107 & 217 & $\mathrm{DL}$ & 3 & $9 / 17 / 97$ & 13168 & $\begin{array}{l}60 \\
\text { Total Weight }\end{array}$ & $\begin{array}{r}7.4 \\
7.4\end{array}$ & S97T002068 \\
\hline B-107 & .217 & $\mathrm{LL}$ & 3 & $9 / 17 / 97$ & 13478 & $\begin{array}{l}60 \\
\text { Total Weight }\end{array}$ & $\begin{array}{r}16.4 \\
16.4\end{array}$ & S97T002294 \\
\hline $\begin{array}{l}\text { B-107 } \\
\text { B-107 }\end{array}$ & $\begin{array}{l}217 \\
217\end{array}$ & $\begin{array}{l}\text { Salt } \\
\text { Salt }\end{array}$ & $\begin{array}{l}1 \\
1\end{array}$ & $\begin{array}{l}9 / 18 / 97 \\
9 / 18 / 97\end{array}$ & $\begin{array}{l}13175 \\
13177\end{array}$ & $\begin{array}{l}60 \\
60 \\
\text { Total Weight }\end{array}$ & $\begin{array}{c}95 \\
65.5 \\
160.5\end{array}$ & $\begin{array}{l}\text { S97T002070 } \\
\text { S97T002072 }\end{array}$ \\
\hline $\begin{array}{l}\text { B-107 } \\
\text { B-107 } \\
\text { B-107 } \\
\text { B-107 } \\
\text { B-107 }\end{array}$ & $\begin{array}{l}217 \\
217 \\
217 \\
217 \\
217\end{array}$ & $\begin{array}{l}\text { Sludge } \\
\text { Sludge } \\
\text { Sludge } \\
\text { Sludge } \\
\text { Sludge }\end{array}$ & $\begin{array}{l}2 \\
2 \\
3 \\
3 \\
3\end{array}$ & $\begin{array}{l}9 / 18 / 97 \\
9 / 18 / 97 \\
9 / 17 / 97 \\
9 / 18 / 97 \\
9 / 18 / 97\end{array}$ & $\begin{array}{l}13174 \\
13176 \\
12893 \\
13178 \\
13179\end{array}$ & $\begin{array}{c}60 \\
60 \\
250 \\
60 \\
60 \\
\text { Total Weight }\end{array}$ & $\begin{array}{c}115.4 \\
109 \\
52.1 \\
111.1 \\
60.3 \\
447.9\end{array}$ & $\begin{array}{l}\text { S97T002076 } \\
\text { S97T002074 } \\
\text { S97T002067 } \\
\text { S97T002083 } \\
\text { S97T002081 }\end{array}$ \\
\hline B-107 & 218 & DL & 4 & 9/17/97 & 13171 & $\begin{array}{c}60 \\
\text { Total Weight }\end{array}$ & $\frac{42.2}{42.2}$ & S97T002116 \\
\hline B-107 & 218 & LL & 4 & $9 / 17 / 97$ & 12367 & $\begin{array}{l}125 \\
\text { Total Weight }\end{array}$ & $\frac{80.6}{80.6}$ & S97T002296 \\
\hline $\begin{array}{l}\text { B-107 } \\
\text { B-107 }\end{array}$ & $\begin{array}{l}218 \\
218\end{array}$ & $\begin{array}{l}\text { Salt } \\
\text { Salt }\end{array}$ & $\begin{array}{l}1 \\
4\end{array}$ & $\begin{array}{l}9 / 17 / 97 \\
9 / 17 / 97\end{array}$ & $\begin{array}{l}13169 \\
13172\end{array}$ & $\begin{array}{c}60 \\
60 \\
\text { Total Weight }\end{array}$ & $\begin{array}{c}2.9 \\
7 \\
9.9\end{array}$ & $\begin{array}{l}\text { S97T002105 } \\
\text { S97T002113 }\end{array}$ \\
\hline B-107 & 218 & Sludge & 2 & 9/17/97 & 13170 & $\begin{array}{c}60 \\
\text { Total Weight }\end{array}$ & $\begin{array}{r}71.9 \\
71.9\end{array}$ & S97T002111 \\
\hline Tank \# & Core \# & Matrix & Segment \# & Date & Jar/Vial \# & Vial Size (mLs) & Net Wt\# Found (gms) & Laboratory \# \\
\hline B-108 & 172 & $\mathrm{DL}$ & 1 & $10 / 16 / 96$ & 11410 & $\begin{array}{c}40 \\
\text { Total Weight }\end{array}$ & $\frac{6.4}{6.4}$ & S96T005522 \\
\hline $\begin{array}{l}\text { B-108 } \\
\text { B-108 }\end{array}$ & $\begin{array}{l}172 \\
172\end{array}$ & $\begin{array}{l}\text { FB } \\
\text { FB }\end{array}$ & $\begin{array}{l}\text { Folk } \\
\text { Fblk }\end{array}$ & $\begin{array}{l}10 / 16 / 96 \\
10 / 18 / 96\end{array}$ & $\begin{array}{l}11516 \\
11501\end{array}$ & $\begin{array}{l}250 \\
60 \\
\text { Total Weight }\end{array}$ & $\begin{array}{c}119.2 \\
65.7 \\
184.9\end{array}$ & $\begin{array}{l}\text { S96T005462 } \\
\text { S96T005464 }\end{array}$ \\
\hline
\end{tabular}


Vials in 11A1 and 11A2

\begin{tabular}{|c|c|c|c|c|c|c|c|c|}
\hline Tank\# & Core \# & Matrix & Segment\# & Date & Jar/Vial \# & Vial Size (mLs) & Net Wt\# Found (gms) & Laboratory \# \\
\hline B-108 & 172 & Salt & 1 & $10 / 21 / 96$ & 11639 & 60 & 76.1 & S96T005509 \\
\hline B-108 & 172 & Salt & 1 & $10 / 30 / 96$ & 11761 & 60 & 38.4 & S96T005508 \\
\hline \multirow[t]{2}{*}{ B-108 } & 172 & Salt & 2 & $10 / 21 / 96$ & 11478 & 60 & 17.7 & S96T005504 \\
\hline & & & & & & Total Weight & 132.2 & \\
\hline B-108 & 173 & Salt & 2 & $10 / 19 / 96$ & 11484 & $\begin{array}{c}60 \\
\text { Total Weight }\end{array}$ & $\frac{64.7}{64.7}$ & S96T005476 \\
\hline B-108 & 173 & Sludge & 1 & $10 / 18 / 96$ & 11505 & 60 & 12.4 & S96T005475 \\
\hline B-108 & 173 & Sludge & 1 & $10 / 18 / 96$ & 11506 & 60 & 58.7 & S96T005467 \\
\hline \multirow[t]{2}{*}{ B-108 } & 173 & Sludge & Comp & $10 / 17 / 96$ & 11507 & 60 & 22.8 & S96T005483 \\
\hline & & & & & & Total Weight & 939 & \\
\hline
\end{tabular}

$\begin{array}{lllllll}\text { Tank \# Core \# } & \text {. Matrix } & \text { Segment \# } & \text { Date } & \text { Jar/Vial \# } & \text { Vial Size (mLs) Net Wt\# Found (gms) Laboratory \# }\end{array}$

\begin{tabular}{|c|c|c|c|c|c|c|c|c|}
\hline B-109 & 169 & Salt & 2 & $10 / 7 / 96$ & 11486 & $\begin{array}{l}60 \\
\text { Total Weight }\end{array}$ & $\begin{array}{l}98.8 \\
98.8\end{array}$ & S96T005105 \\
\hline $\begin{array}{l}\text { B-109 } \\
\text { B-109 }\end{array}$ & $\begin{array}{l}170 \\
170\end{array}$ & $\begin{array}{l}\text { FB } \\
\text { FB }\end{array}$ & $\begin{array}{l}\text { Fblk } \\
\text { Fblk }\end{array}$ & $\begin{array}{l}9 / 16 / 96 \\
10 / 7 / 96\end{array}$ & $\begin{array}{l}11187 \\
11487\end{array}$ & $\begin{array}{c}250 \\
60 \\
\text { Total Weight }\end{array}$ & $\begin{array}{c}130 \\
65.6 \\
195.6\end{array}$ & $\begin{array}{l}\text { S96T005115 } \\
\text { S96ro05117 }\end{array}$ \\
\hline $\begin{array}{l}\text { B-109 } \\
\text { B-109 } \\
\text { B-109 } \\
\text { B-109 } \\
\text { B-109 } \\
\text { B-109 }\end{array}$ & $\begin{array}{l}170 \\
170 \\
170 \\
170 \\
170 \\
170\end{array}$ & $\begin{array}{l}\text { Salt } \\
\text { Salt } \\
\text { Salt } \\
\text { Salt } \\
\text { Salt } \\
\text { Salt }\end{array}$ & $\begin{array}{l}1 \\
2 \\
2 \\
2 \\
2 \\
2\end{array}$ & $\begin{array}{l}10 / 7 / 96 \\
9 / 16 / 96 \\
9 / 16 / 96 \\
9 / 16 / 96 \\
9 / 16 / 96 \\
10 / 8 / 96\end{array}$ & $\begin{array}{l}11488 \\
11181 \\
11360 \\
11363 \\
11364 \\
11390\end{array}$ & $\begin{array}{c}60 \\
250 \\
60 \\
60 \\
60 \\
60 \\
\text { Total Weight }\end{array}$ & $\begin{array}{c}28.5 \\
144.7 \\
17.7 \\
36.9 \\
9.7 \\
110 \\
347.5\end{array}$ & $\begin{array}{l}\text { S96T005120 } \\
\text { S96T005128 } \\
\text { S96T005130 } \\
\text { S96T005129 } \\
\text { S96T005127 } \\
\text { S96T005134 }\end{array}$ \\
\hline
\end{tabular}

\begin{tabular}{lllcrcccc} 
Tank \# & Core \# & Matrix & Segment \# & \multicolumn{1}{c}{ Date } & Jar/Vial \# & Vial Size (mLs) & Net Wt\# Found (gms) & Laboratory \# \\
\hline B-203 & 115 & Sludge & 1 & $12 / 13 / 95$ & 8814 & 60 & 66.9 & S95T003847 \\
B-203 & 115 & Sludge & 1 & $12 / 13 / 95$ & 8815 & 60 & 69.4 & S95T003846 \\
B-203 & 115 & Sludge & 10 & $1 / 18 / 96$ & 9089 & 60 & 77.7 & S95T003941 \\
B-203 & 115 & Sludge & 10 & $1 / 18 / 96$ & 9090 & 60 & 75 & S95T003946 \\
B-203 & 115 & Sludge & 4 & $11 / 29 / 95$ & 8489 & 250 & 225.8 & S95T003845
\end{tabular}

17-Mar-98

Page 35 of 114 
Vials in 11A1 and 11A2

\begin{tabular}{|c|c|c|c|c|c|c|c|c|}
\hline Tank \# & Core\# & Matrix & Segment \# & Date & Jar/Vial \# & Vial Size (mLs) & Net Wt\# Found (gms) & Laboratory \# \\
\hline B-203 & 115 & Sludge & 4 & $12 / 13 / 95$ & 8816 & 60 & 64.4 & S95T003849 \\
\hline B-203 & 115 & Sludge & 5 & $1 / 17 / 96$ & 9081 & 60 & 59 & S95T003922 \\
\hline B-203 & 115 & Sludge & 5 & $1 / 17 / 96$ & 9082 & 60 & 60.9 & S95T003923 \\
\hline B-203 & 115 & Sludge & 6 & $1 / 17 / 96$ & 9020 & 60 & 76.3 & S95T003942 \\
\hline B-203 & 115 & Sludge & 6 & $1 / 17 / 96$ & 9021 & 60 & 72 & S95T003937 \\
\hline B-203 & 115 & Sludge & 7 & $1 / 17 / 96$ & 9083 & 60 & 48.7 & S95T003938 \\
\hline B-203 & 115 & Sludge & 7 & $1 / 17 / 96$ & 9084 & 60 & 78.8 & S95T003943 \\
\hline B-203 & 115 & Sludge & 8 & $1 / 17 / 96$ & 9085 & 60 & 75.4 & S95T003944 \\
\hline B-203 & 115 & Sludge & 8 & $1 / 17 / 96$ & 9086 & 60 & 81.8 & S95T003939 \\
\hline B-203 & 115 & Sludge & 9 & $1 / 17 / 96$ & 9087 & 60 & 76.1 & S95T003945 \\
\hline \multirow[t]{2}{*}{ B-203 } & 115 & Sludge & 9 & $1 / 17 / 96$ & 9088 & 60 & 79.2 & S95T003940 \\
\hline & & & & & & Total Weight & 1257.4 & \\
\hline B-203 & 120 & Sludge & 10 & $1 / 2 / 96$ & 8909 & 60 & 53.6 & S95T004120 \\
\hline B-203 & 120 & Sludge & 10 & $1 / 2 / 96$ & 8910 & 60 & 60.6 & S95T004129 \\
\hline B-203 & 120 & Sludge & 13 & $1 / 2 / 96$ & 8911 & 60 & 39.1 & S95T004121 \\
\hline B-203 & 120 & Sludge & 13 & $1 / 2 / 96$ & 8912 & 60 & 65.6 & S95T004130 \\
\hline B-203 & 120 & Sludge & 14 & $1 / 2 / 96$ & 8913 & 60 & 74.8 & S95T004122 \\
\hline B-203 & 120 & Sludge & 14 & $1 / 2 / 96$ & 8914 & 60 & 70.5 & S95T004131 \\
\hline B-203 & 120 & Sludge & 4 & $12 / 16 / 95$ & 8508 & 250 & 162.3 & S95T003965 \\
\hline B-203 & 120 & Sludge & 4 & $1 / 2 / 96$ & 8819 & 60 & 23.3 & S95T004114 \\
\hline B-203 & 120 & Sludge & 4 & $1 / 2 / 96$ & 8897 & 60 & 62.6 & S95T004123 \\
\hline B-203 & 120 & Sludge & 5 & $1 / 2 / 96$ & 8898 & 60 & 22.8 & S95T004115 \\
\hline B-203 & 120 & Sludge & 5 & $1 / 2 / 96$ & 8899 & 60 & 8 & S95T004124 \\
\hline B-203 & 120 & Sludge & 6 & $1 / 2 / 96$ & 8900 & 60 & 57 & S95T004125 \\
\hline B-203 & 120 & Sludge & 6 & $1 / 2 / 96$ & 8901 & 60 & 29 & S95T004116 \\
\hline B-203 & 120 & Sludge & 7 & $1 / 2 / 96$ & 8902 & 60 & 62.1 & S95T004117 \\
\hline B-203 & 120 & Sludge & 7 & $1 / 3 / 96$ & 8903 & 60 & 49.7 & S95T04126 \\
\hline B-203 & 120 & Sludge & 8 & $1 / 2 / 96$ & 8904 & 60 & 9.9 & S95T004118 \\
\hline B-203 & $120^{\circ}$ & Sludge & 8 & $1 / 2 / 96$ & 8905 & 60 & 73.9 & S95T004127 \\
\hline B-203 & 120 & Sludge & 9 & $1 / 2 / 96$ & 8906 & 60 & 60.7 & S95T004119 \\
\hline \multirow[t]{2}{*}{ B-203 } & 120 & Sludge & 9 & $1 / 2 / 96$ & 8908 & 60 & 50.6 & S95T004128 \\
\hline & & & & & & Total Weight & 1036.1 & \\
\hline B-203 & 122 & DL & 1 & $1 / 8 / 96$ & 8958 & 60 & 51.3 & S95T004005 \\
\hline
\end{tabular}


Vials in 11A1 and 11A2

\begin{tabular}{|c|c|c|c|c|c|c|c|c|}
\hline Tank \# & Core \# & Matrix & Segment \# & Date & Jar/Vial \# & Vial Size (mLs) & Net Wt\# Found (gms) & Laboratory \# \\
\hline B-203 & 122 & $\mathrm{DL}$ & 3 & $1 / 8 / 96$ & 8413 & $\begin{array}{l}40 . \\
\text { Total Weight }\end{array}$ & $\frac{10.3}{61.6}$ & S95T004004 \\
\hline B-203 & 122 & Sludge & 10 & $1 / 11 / 96$ & 9008 & 60 & 42.4 & \$96T000045 \\
\hline B-203 & 122 & Sludge & 10 & $1 / 11 / 96$ & 9009 & 60 & 56.1 & S96T000051 \\
\hline B-203 & 122 & Sludge & 11 & $1 / 11 / 96$ & 9010 & 60 & 51.5 & S96T000046 \\
\hline B-203 & 122 & Sludge & 11 & $1 / 11 / 96$ & 9011 & 60 & 31.9 & S96T000052 \\
\hline B-203 & 122 & Sludge & 12 & $1 / 12 / 96$ & 9012 & 60 & 66.3 & S96T000053 \\
\hline B-203 & 122 & Sludge & 12 & $1 / 12 / 96$ & 9013 & 60 & 61.1 & S96T000047 \\
\hline B-203 & 122 & Sludge & 13 & $1 / 12 / 96$ & 9014 & 60 & 43.9 & S96T000048 \\
\hline B-203 & 122 & Sludge & 13 & $1 / 12 / 96$ & 9015 & 60 & 37.6 & S96T000054 \\
\hline B-203 & 122 & Sludge & 14 & $1 / 12 / 96$ & 9016 & 60 & 62.2 & S96T000049 \\
\hline B-203 & 122 & Sludge & 14 & $1 / 12 / 96$ & 9017 & 60 & 62.7 & S96T000055 \\
\hline B-203 & 122 & Sludge & 3 & $1 / 8 / 96$ & 8959 & 60 & 61.1 & S95T004018 \\
\hline B-203 & 122 & Sludge & 4 & $1 / 9 / 96$ & 8960 & 60 & 36.3 & S95T004016 \\
\hline B-203 & 122 & Sludge & 4 & $1 / 9 / 96$ & 8961 & 60 & 59.2 & S95T004019 \\
\hline B-203 & 122 & Sludge & 5 & $1 / 9 / 96$ & 8962 & 60 & 75.6 & S95T008962 \\
\hline B-203 & 122 & Sludge & 5 & $1 / 9 / 96$ & 8963 & 60 & 77.7 & S9ST004020 \\
\hline B-203 & 122 & Sludge & 6 & $1 / 9 / 96$ & 8966 & 60 & 70.7 & S95T004224 \\
\hline B-203 & 122 & Sludge & 6 & $1 / 9 / 96$ & 8971 & 60 & 70.7 & S95T004230 \\
\hline B-203 & 122 & Sludge & 7 & $1 / 9 / 96$ & 8972 & 60 & 46 & S95T004225 \\
\hline B-203 & 122 & Sludge & 7 & $1 / 9 / 96$ & 8973 & 60 & 70.4 & S95T004231 \\
\hline B-203 & 122 & Sludge & 8 & $1 / 9 / 96$ & 8974 & 60 & 52.4 & \$95T004226 \\
\hline B-203 & 122 & Sludge & 8 & $1 / 9 / 96$ & 8975 & 60 & 74.8 & S95T004232 \\
\hline B-203 & 122 & Sludge & 9 & $1 / 11 / 96$ & 8978 & 60 & 59.1 & S96T000050 \\
\hline B-203 & 122 & Sludge & 9 & $1 / 11 / 96$ & 8979 & 60 & 54.2 & S96T000044 \\
\hline B-203 & 122 & Sludge & Comp & $12 / 18 / 95$ & 8534 & 250 & $\frac{159.3}{1093 ?}$ & S95T004000 \\
\hline
\end{tabular}

\begin{tabular}{lllcccccc} 
Tank \# & Core \# & Matrix & Segment \# & Date & Jar/Vial \# & Vial Size (mLs) & Net Wt\# Found (gms) & Laboratory \# \\
\hline B-204 & 112 & DL & 1 & $10 / 27 / 95$ & 8027 & 40 & 43.7 & S95T002974 \\
B-204 & 112 & DL & 2 & $10 / 31 / 95$ & 8029 & 40 & 43.4 & S95T002983 \\
B-204 & 112 & DL & 2 & $10 / 31 / 95$ & 8030 & 40 & 41.8 & S95T002983 \\
B-204 & 112 & DL & 2 & $10 / 31 / 95$ & 8031 & 40 & 22.2 & S95T002983
\end{tabular}

17-Mar-98 
Vials in 11A1 and 11A2

\begin{tabular}{|c|c|c|c|c|c|c|c|c|}
\hline Tank \# & Core\# & Matrix & Segment \# & Date & Jar/Vial \# & Vial Size (mLs) & Net Wt\# Found (gms) & Laboratory \# \\
\hline & & & & & & Total Weight & 151.1 & \\
\hline B-204 & 112 & LL & 2 & $10 / 31 / 95$ & 8032 & 40 & 40.5 & S95T003375 \\
\hline B-204 & 112 & LL & 2 & $10 / 31 / 95$ & 8033 & 40 & 39.5 & S95T003375 \\
\hline B-204 & 112 & LL & 2 & $10 / 31 / 95$ & 8388 & 40 & 30.7 & S95T003375 \\
\hline \multirow[t]{2}{*}{ B-204 } & 112 & LL & 8 & $10 / 18 / 95$ & 7681 & 40 & 7.4 & \\
\hline & & & & & & Total Weight & 118.1 & \\
\hline B-204 & 112 & Sludge & 10 & $10 / 18 / 95$ & 8226 & 250 & 73.5 & S95T003003 \\
\hline B-204 & 112 & Sludge & 10 & $10 / 18 / 95$ & 8229 & 250 & 31 & S95T003004 \\
\hline B-204 & 112 & Sludge & 10 & $11 / 9 / 95$ & 8556 & 60 & 74.7 & S95T003027 \\
\hline B-204 & 112 & Sludge & 10 & $11 / 9 / 95$ & 8557 & 60 & 68.7 & S95T003028 \\
\hline B-204 & 112 & Sludge & 11 & $10 / 19 / 95$ & 8232 & 250 & 30.9 & S95T003006 \\
\hline B-204 & 112 & Sludge & 11 & $10 / 19 / 95$ & 8233 & 250 & 60.5 & S95T003005 \\
\hline B-204 & 112. & Sludge & 11 & $11 / 9 / 95$ & 8202 & 60 & 73.1 & S95T003029 \\
\hline B-204 & 112 & Sludge & 11 & $11 / 9 / 95$ & 8203 & 60 & 61.5 & S95T003030 \\
\hline B-204 & 112 & Sludge & 12 & $10 / 19 / 95$ & 8234 & 250 & 47.6 & S95T003008 \\
\hline B-204 & 112 & Sludge & 12 & $10 / 19 / 95$ & 8235 & 250 & 74.9 & S95T003007 \\
\hline B-204 & 112 & Sludge & 12 & 11/9/95 & 8204 & 60 & 67.7 & S95T003031 \\
\hline B-204 & 112 & Sludge & 12 & $11 / 9 / 95$ & 8205 & 60 & 65.5 & S95T003032 \\
\hline B-204 & 112 & Sludge & 13 & $10 / 19 / 95$ & 8237 & 250 & 54.7 & S95T003010 \\
\hline B-204 & 112 & Sludge & 13 & $10 / 19 / 95$ & 8239 & 250 & 62.8 & S95T002943 \\
\hline B-204 & 112 & Sludge & 13 & $11 / 9 / 95$ & 8206 & 60 & 54.3 & S95T003033 \\
\hline B-204 & 112 & Sludge & 13 & $11 / 9 / 95$ & 8207 & 60 & 73.3 & S95T003034 \\
\hline B-204 & 112 & Sludge & 14 & $10 / 18 / 95$ & 8230 & 250 & 40.5 & S95T003012 \\
\hline B-204 & 112 & Sludge & 14 & $10 / 18 / 95$ & 8231 & 250 & 67.5 & S95T003011 \\
\hline B-204 & 112 & Sludge & 14 & $11 / 9 / 95$ & 8200 & 60 & 19.1 & S95T003035 \\
\hline B-204 & 112 & Sludge & 14 & $11 / 9 / 95$ & 8201 & 60 & 69.7 & S95T003036 \\
\hline B-204 & 112 & Sludge & 2 & $10 / 30 / 95$ & 8028 & 40 & 15.7 & S95T002978 \\
\hline B-204 & 112 & Sludge & 3 & $10 / 16 / 95$ & 8159 & 250 & 22.3 & S95T002988 \\
\hline B-204 & 112 & Sludge & 3 & $10 / 16 / 95$ & 8164 & 250 & 51.2 & S95T002989 \\
\hline B-204 & 112 & Sludge & 3 & $11 / 1 / 95$ & 8560 & 60 & 68.7 & S95T003013 \\
\hline B-204 & 112 & Sludge & 3 & 11/1/95 & 8561 & 60 & 65.2 & S95T003014 \\
\hline B-204 & 112 & Sludge & 4 & $10 / 16 / 95$ & 8165 & 250 & 42.3 & S95T002992 \\
\hline B-204 & 112 & Sludge & 4 & $10 / 16 / 95$ & 8166 & 250 & 28.4 & S95T002991 \\
\hline
\end{tabular}


Vials in 11A1 and 11A2

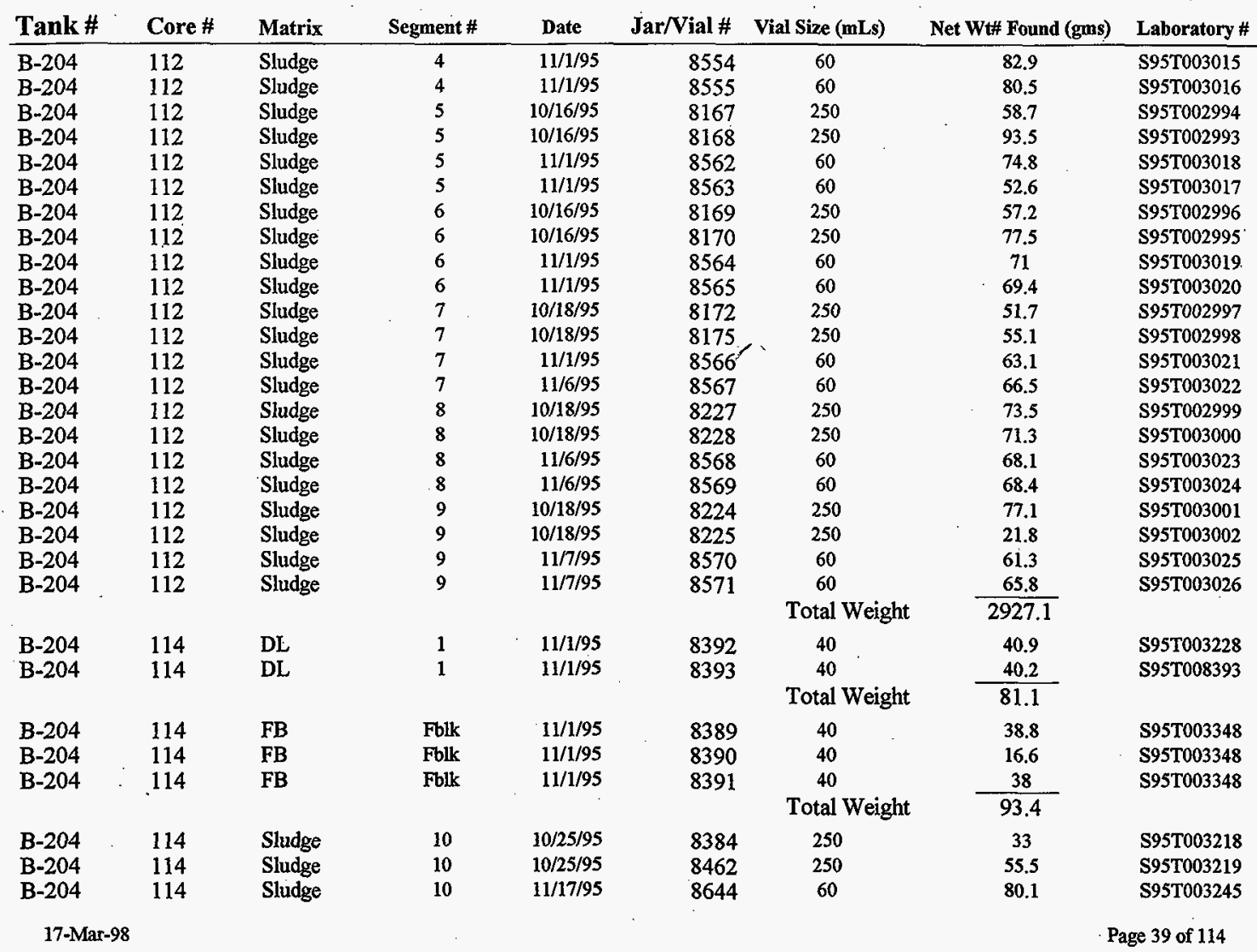


Vials in 11A1 and 11A2

\begin{tabular}{|c|c|c|c|c|c|c|c|c|}
\hline Tank \# & Core \# & Matrix & Segment \# & Date & Jar/Vial \# & Vial Size (mLs) & Net Wt\# Found (gms) & Laboratory \# \\
\hline B-204 & 114 & Sludge & 10 & $11 / 17 / 95$ & 8645 & 60 & 70.9 & S95T003246 \\
\hline B-204 & 114 & Sludge & 11 & $10 / 25 / 95$ & 8465 & 250 & 37.3 & S95T003220 \\
\hline B-204 & 114 & Sludge & 11 & $10 / 25 / 95$ & 8466 & 250 & 32.4 & S95T003221 \\
\hline B-204 & 114 & Sludge & 11 & $11 / 17 / 95$ & 8646 & 60 & 68.7 & \$95T003247 \\
\hline B-204 & 114 & Sludge & 11 & $11 / 17 / 95$ & 8647 & 60 & 79.3 & S95T003248 \\
\hline B-204 & 114 & Sludge & 12 & $10 / 25 / 95$ & 8461 & 250 & 70.6 & S95T003223 \\
\hline B-204 & 114 & Sludge & 12 & $10 / 25 / 95$ & 8463 & 250 & 25.4 & S95T003222 \\
\hline B-204 & 114 & Sludge & 12 & $11 / 20 / 95$ & 8648 & 60 & 73.2 & S95T003250 \\
\hline B-204 & 114 & Sludge & 12 & $11 / 20 / 95$ & 8663 & 60 & 74.7 & S95T003249 \\
\hline B-204 & 114 & Sludge & 13 & $10 / 26 / 95$ & 8467 & 250 & 75.2 & S95T003225 \\
\hline B-204 & 114 & Sludge & 13 & $10 / 26 / 95$ & 8484 & 250 & 42.9 & S95T003224 \\
\hline B-204 & 114 & Sludge & 13 & $11 / 22 / 95$ & 8674 & 60 & 67.4 & S95T003251 \\
\hline B-204 & 114 & Sludge & 13 & $11 / 22 / 95$ & 8675 & 60 & 65.8 & S95T003252 \\
\hline B-204 & 114 & Sludge & 13 & $11 / 22 / 95$ & 8678 & 60 & 69.9 & S95T003254 \\
\hline B-204 & 114 & Sludge & 14 & $10 / 26 / 95$ & 8468 & 250 & 57 & \$95T003226 \\
\hline B-204 & 114 & Sludge & 14 & $10 / 26 / 95$ & 8470 & 250 & 38.8 & S95T003227 \\
\hline B-204 & 114 & Sludge & 14 & $11 / 22 / 95$ & 8677 & 60 & 71.9 & S95T003253 \\
\hline B-204 & 114 & Sludge & 2 & $10 / 19 / 95$ & 8247 & 250 & 37.9 & \$95T003202 \\
\hline B-204 & 114 & Sludge & 2 & $11 / 9 / 95$ & 8558 & 60 & 56.1 & S95T003229 \\
\hline B-204 & 114 & Sludge & 2 & $11 / 9 / 95$ & 8559 & 60 & 62.4 & S95T003230 \\
\hline B-204 & 114 & Sludge & 3 & $10 / 23 / 95$ & 8380 & 250 & 21.8 & S95T003204 \\
\hline B-204 & 114 & Sludge & 3 & $11 / 9 / 95$ & 8572 & 60 & 65.7 & S95T003232 \\
\hline B-204 & 114 & Sludge & 3 & $11 / 9 / 95$ & 8573 & 60 & 70.7 & S95T003231 \\
\hline B-204 & 114 & Sludge & 4 & $10 / 23 / 95$ & 8382 & 250 & 41.8 & S95T003206 \\
\hline B-204 & 114 & Sludge & 4 & $10 / 23 / 95$ & 8383 & 250 & 36.5 & S95T003207 \\
\hline B-204 & 114 & Sludge & 4 & $11 / 9 / 95$ & 8633 & 60 & 48.4 & S95T003234 \\
\hline B-204 & 114 & Sludge & 4 & $11 / 9 / 95$ & 8634 & 60 & 26.3 & S95T003233 \\
\hline B-204 & 114 & Sludge & 5 & $10 / 23 / 95$ & 8244 & 250 & 57.8 & S95T003208 \\
\hline B-204 & 114 & Sludge & 5 & $10 / 23 / 95$ & 8245 & 250 & 43.5 & S95T003209 \\
\hline B-204 & 114 & Sludge & 5 & $11 / 14 / 95$ & 8635 & 60 & 60 & S95T003235 \\
\hline B-204 & 114 & Sludge & 5 & $11 / 14 / 95$ & 8636 & 60 & 63.8 & S95T003236 \\
\hline B-204 & 114 & Sludge & 6 & $10 / 23 / 95$ & 8242 & 250 & 70.2 & S95T003211 \\
\hline B-204 & 114 & Sludge & 6 & $10 / 23 / 95$ & 8243 & 250 & 55.4 & S95T003210 \\
\hline B-204 & 114 & Sludge & 6 & $11 / 13 / 95$ & 8637 & 60 & 60.1 & S95T003238 \\
\hline
\end{tabular}


Vials in 11A1 and 11A2

\begin{tabular}{|c|c|c|c|c|c|c|c|c|}
\hline Tank \# & Core\# & Matrix & Segment \# & Date & Jar/Vial \# & Vial Size (mLs) & Net Wt\# Found (gms) & Laboratory \# \\
\hline B-204 & 114 & Sludge & 6 & $11 / 13 / 95$ & 8638 & 60 & 59.8 & S95T003237 \\
\hline B-204 & 114 & Sludge & 7 & $10 / 24 / 95$ & 8457 & 250 & 28.3 & S95T003212 \\
\hline B-204 & 114 & Sludge & 7 & $10 / 24 / 95$ & 8458 & 250 & 35.2 & S95T003213 \\
\hline B-204 & 114 & Sludge & 7 & $11 / 13 / 95$ & 8639 & 60 & 70.7 & S95T003239 \\
\hline B-204 & 114 & Sludge & 7 & $11 / 13 / 95$ & 8640 & 60 & 71.7 & S95T003240 \\
\hline B-204 & 114 & Sludge & 8 & $10 / 24 / 95$ & 8379 & 250 & 61.1 & S95T003215 \\
\hline B-204 & 114 & Sludge & 8 & $11 / 9 / 95$ & 8574 & 60 & 67.7 & S95T00324I \\
\hline B-204 & 114 & Sludge & 8 & $11 / 9 / 95$ & 8575 & 60 & 59.1 & S95T003242 \\
\hline B-204 & 114 & Sludge & 9 & $10 / 24 / 95$ & 8459 & 250 & 48 & S9ST003216 \\
\hline B-204 & 114 & Sludge & 9 & $10 / 24 / 95$ & 8460 & 250 & 55.6 & S95T003217 \\
\hline B-204 & 114 & Sludge & 9 & $11 / 10 / 95$ & 8580 & 60 & 63.2 & S95T003244 \\
\hline \multirow[t]{2}{*}{ B-204 } & 114 & Sludge & 9 & $11 / 10 / 95$ & 8581 & 60 & 67.5 & S95T003243 \\
\hline & & & & & & Total Weigh & 2756.3 & \\
\hline
\end{tabular}

\begin{tabular}{|c|c|c|c|c|c|c|c|c|}
\hline Tank \# & Core \# & Matrix & Segment \# & Date & Jar/Vial \# & Vial Size (mLs) & Net Wtt Found (gms) & Laboratory \# \\
\hline BX-101 & R1 & Sludge & Auger & $6 / 23 / 94$ & 6045 & 125 & 12.8 & \\
\hline \multirow[t]{2}{*}{ BX-101 } & $\mathrm{R} 1$ & Sludge & Auger & $6 / 23 / 94$ & 6046 & 125 & 47.9 & \\
\hline & & & & & & Total Weight & 60.7 & \\
\hline BX-101 & R7 & Sludge & Auger & $6 / 27 / 94$ & 6058 & 125 & 11.1 & \\
\hline \multirow[t]{2}{*}{ BX-101 } & R7 & Sludge & Auger & $6 / 27 / 94$ & 6059 & 125 & 6.8 & \\
\hline & & & & & & Total Weight & 17.9 & \\
\hline
\end{tabular}

\begin{tabular}{|c|c|c|c|c|c|c|c|c|}
\hline Tank \# & Core \# & Matrix & Segment \# & Date & Jar/Vial \# & Vial Size (mLs) & Net Wt\# Found (gms) & Laboratory \# \\
\hline $\begin{array}{l}\text { BX-103 } \\
\text { BX-103 }\end{array}$ & $\begin{array}{l}86 \\
86\end{array}$ & $\begin{array}{l}\mathrm{DL} \\
\mathrm{DL}\end{array}$ & $\begin{array}{l}1 \\
1\end{array}$ & $\begin{array}{r}6 / 5 / 95 \\
1 / 24 / 96\end{array}$ & $\begin{array}{l}7183 \\
8426\end{array}$ & $\begin{array}{c}40 \\
40 \\
\text { Total Weight }\end{array}$ & $\begin{array}{l}36.7 \\
47.6 \\
84.3\end{array}$ & S95T001030 \\
\hline $\begin{array}{l}\text { BX-103 } \\
\text { BX-103 } \\
\text { BX-103 } \\
\text { BX-103 }\end{array}$ & $\begin{array}{l}86 \\
86 \\
86 \\
86\end{array}$ & $\begin{array}{l}\text { Sludge } \\
\text { Sludge } \\
\text { Sludge } \\
\text { Sludge }\end{array}$ & $\begin{array}{l}1 \\
2 \\
2 \\
2\end{array}$ & $\begin{array}{r}6 / 5 / 95 \\
6 / 5 / 95 \\
6 / 5 / 95 \\
9 / 15 / 97\end{array}$ & $\begin{array}{r}7181 \\
7182 \\
7185 \\
13157\end{array}$ & $\begin{array}{c}40 \\
40 \\
40 \\
60 \\
\text { Total Weight }\end{array}$ & $\begin{array}{c}10.9 \\
56 \\
49.3 \\
33.2 \\
149.4\end{array}$ & $\begin{array}{l}\text { S95T001027 } \\
\text { S95T001029 } \\
\text { S95T001028 } \\
\text { S95T001693 }\end{array}$ \\
\hline
\end{tabular}




\section{Vials in 11A1 and $11 \mathrm{A2}$}

\begin{tabular}{|c|c|c|c|c|c|c|c|c|}
\hline Tank \# & Core\# & Matrix & Segment \# & Date & Jar/Vial \# & Vial Size (mLs) & Net Wt\# Found (gms) & Laboratory \# \\
\hline $\begin{array}{l}\mathrm{BX}-103 \\
\mathrm{BX}-103\end{array}$ & $\begin{array}{l}87 \\
87\end{array}$ & $\begin{array}{l}\mathrm{DL} \\
\mathrm{DL}\end{array}$ & $\begin{array}{l}1 \\
1\end{array}$ & $\begin{array}{l}6 / 5 / 95 \\
6 / 7 / 95\end{array}$ & $\begin{array}{l}7140 \\
7193\end{array}$ & $\begin{array}{c}250 \\
40 \\
\text { Total Weight }\end{array}$ & $\begin{array}{c}133.8 \\
\frac{41}{174.8}\end{array}$ & S95T001530 \\
\hline $\begin{array}{l}\text { BX-103 } \\
\text { BX-103 }\end{array}$ & $\begin{array}{l}87 \\
87\end{array}$ & $\begin{array}{l}\text { FB } \\
\text { FB }\end{array}$ & $\begin{array}{l}\text { Fblk } \\
\text { Fblk }\end{array}$ & $\begin{array}{l}8 / 3 / 95 \\
8 / 3 / 95\end{array}$ & $\begin{array}{l}7431 \\
7432\end{array}$ & $\begin{array}{l}40 \\
40 \\
\text { Total Weight }\end{array}$ & $\begin{array}{c}39.4 \\
41.6 \\
81\end{array}$ & \\
\hline BX-103 & 87 & LL & 2 & $6 / 5 / 95$ & 7186 & $\begin{array}{l}40 \\
\text { Total Weight }\end{array}$ & $\begin{array}{l}8.5 \\
8.5\end{array}$ & \\
\hline $\begin{array}{l}\text { BX-103 } \\
\text { BX-103 } \\
\text { BX-103 }\end{array}$ & $\begin{array}{l}87 \\
87 \\
87\end{array}$ & $\begin{array}{l}\text { Sludge } \\
\text { Sludge } \\
\text { Sludge }\end{array}$ & $\begin{array}{l}1 \\
2 \\
2\end{array}$ & $\begin{array}{r}6 / 7 / 95 \\
6 / 7 / 95 \\
9 / 16 / 97\end{array}$ & $\begin{array}{r}7195 \\
7194 \\
13163\end{array}$ & $\begin{array}{c}40 \\
40 \\
60 \\
\text { Total Weight }\end{array}$ & $\begin{array}{c}44.4 \\
56.2 \\
71.8 \\
172.4\end{array}$ & $\begin{array}{l}\text { S95T001532 } \\
\text { S95T001533 } \\
\text { S95T001613 }\end{array}$ \\
\hline
\end{tabular}

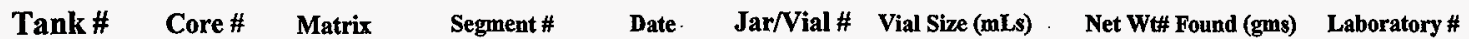

\begin{tabular}{|c|c|c|c|c|c|c|c|}
\hline BX-105 & R6 & Sludge & Auger & 9/3/97 & 13513 & 60. & 62.2 \\
\hline BX-105 & R6 & Sludge & Comp & $9 / 22 / 97$ & 13273 & 40 & 25.9 \\
\hline
\end{tabular}

\begin{tabular}{|c|c|c|c|c|c|c|c|c|}
\hline Tank\# & Core \# & Matrix & Segment \# & Date & Jar/Vial \# & Vial Size (mLs) & Net Wt\# Found (gms) & Laboratory \# \\
\hline BX-106 & & cord & Aug-50 & & 8835 & $\begin{array}{c}250 \\
\text { Total Weight }\end{array}$ & $\begin{array}{l}64 \\
64\end{array}$ & \\
\hline $\begin{array}{l}\text { BX-106 } \\
\text { BX-106 }\end{array}$ & & $\begin{array}{l}\text { Sludge } \\
\text { Sludge }\end{array}$ & $\begin{array}{l}\text { Aug-49 } \\
\text { Aug-50 }\end{array}$ & $\begin{array}{l}1 / 9 / 96 \\
1 / 9 / 96\end{array}$ & $\begin{array}{l}8976 \\
8977\end{array}$ & $\begin{array}{c}60 \\
60 \\
\text { Total Weight }\end{array}$ & $\begin{array}{c}10.8 \\
9.6 \\
20.4\end{array}$ & $\begin{array}{l}\text { S96T000111 } \\
\text { S96T000115 }\end{array}$ \\
\hline $\begin{array}{l}\text { BX-106 } \\
\text { BX-106 }\end{array}$ & $\begin{array}{l}\text { Grab } \\
\text { Grab }\end{array}$ & $\begin{array}{l}\text { GL } \\
\text { GL }\end{array}$ & $\begin{array}{l}1 \\
2\end{array}$ & $\begin{array}{l}8 / 28 / 97 \\
8 / 28 / 97\end{array}$ & $\begin{array}{l}13230 \\
13231\end{array}$ & $\begin{array}{l}40 \\
40\end{array}$ & $\begin{array}{r}55.1 \\
54.1 \\
1092\end{array}$ & $\begin{array}{l}\text { na } \\
\text { na }\end{array}$ \\
\hline
\end{tabular}


Vials in 11A1 and 11A2

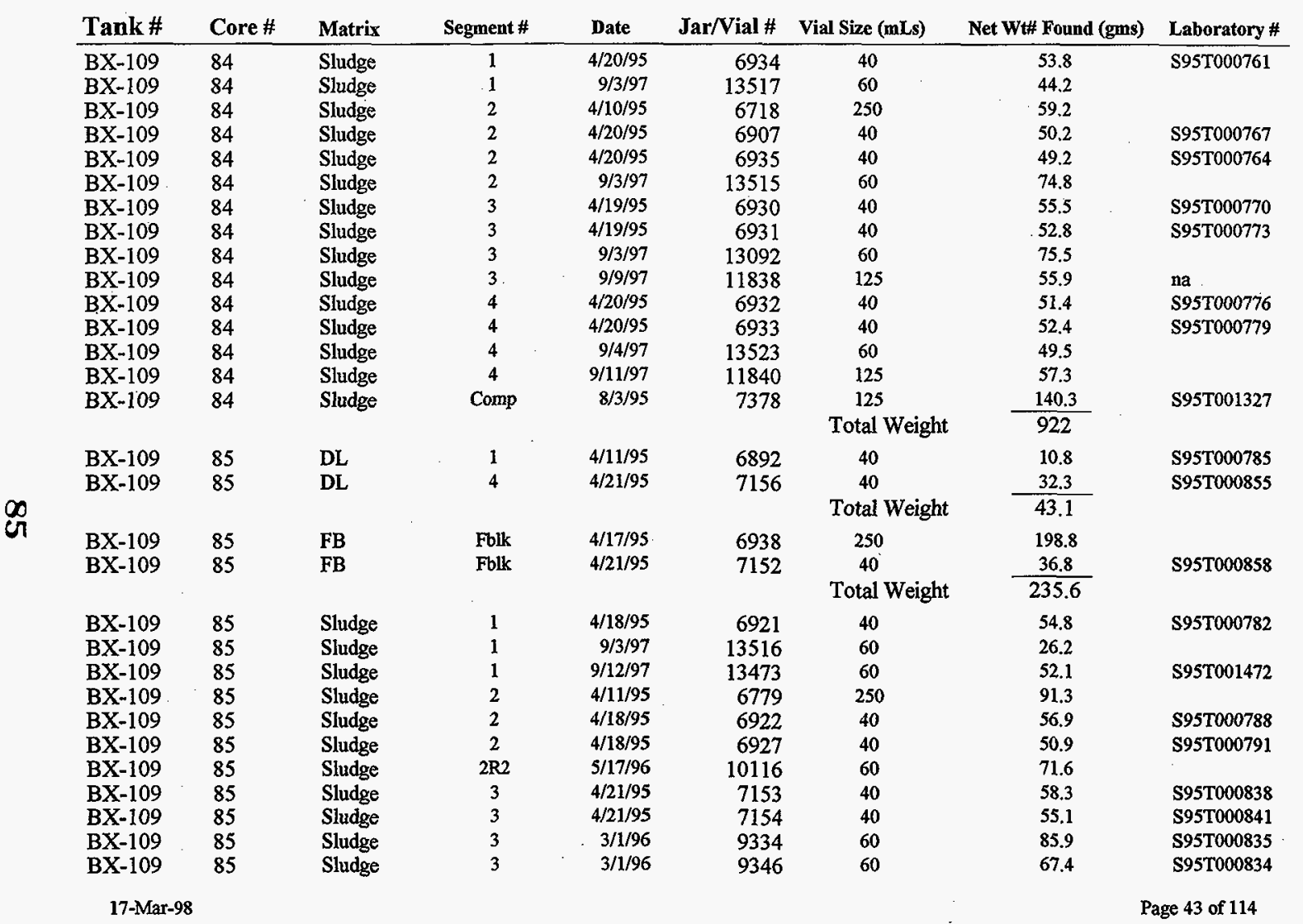




\section{Vials in 11A1 and 11A2}

\begin{tabular}{|c|c|c|c|c|c|c|c|c|}
\hline Tank\# & Core \# & Matrix & Segment \# & Date & Jar/Vial \# & Vial Size (mLs) & Net Wt\# Found (gms) & Laboratory \# \\
\hline BX-109 & 85 & Sludge & 4 & $4 / 21 / 95$ & 7157 & 40 & 56.3 & S95T000849 \\
\hline $\mathrm{BX}-109$ & 85 & Sludge & 4 & $4 / 21 / 95$ & 7158 & 40 & 53.4 & S95T000852 \\
\hline BX-109 & 85 & Sludge & 4 & $9 / 4 / 97$ & 13522 & 60 & 51.5 & \\
\hline BX-109 & 85 & Sludge & 4 & $9 / 11 / 97$ & 11841 & 125 & 14.1 & \\
\hline $\mathrm{BX}-109$ & 85 & Sludge & Comp & $9 / 26 / 97$ & 13445 & 60 & 87.4 & S95T001473 \\
\hline & & & & & & Total Weight & 933.2 & \\
\hline
\end{tabular}

\begin{tabular}{|c|c|c|c|c|c|c|c|c|}
\hline Tank \# & Core\# & Matrix & Segment \# & Date & Jar/Vial \# & Vial Size (mLs) & Net Wt\# Found (gms) & Laboratory \# \\
\hline BX -110 & & Sludge & Aug-46 & $10 / 23 / 95$ & 8476 & 60 & 64.9 & \$95T002947 \\
\hline$B X-110$ & & Sludge & Aug- 46 & $1 / 30 / 96$ & 9212 & 60 & 27.8 & \\
\hline & & & & & & Total Weigh & 92.7 & \\
\hline
\end{tabular}

$\begin{array}{rrrrrrr}\text { BX-110 } & 197 & \text { DL } & 1 & 6 / 16 / 97 & 12244 & 40 \\ \text { BX-110 } & 197 & \text { DL } & 2 & 6 / 16 / 97 & 12246 & 40 \\ \text { BX-110 } & 197 & \text { DL } & 2 A & 6 / 3 / 97 & 12565 & 250 \\ \text { BX-110 } & 197 & \text { DL } & 2 A & 6 / 16 / 97 & 12245 & 40\end{array}$

\begin{tabular}{|c|c|c|c|c|c|c|c|c|}
\hline BX-110 & 197 & Salt & 1 & $6 / 3 / 97$ & 11882 & 125 & 70.4 & S97T001260 \\
\hline$B X-110$ & 197 & Salt & I & $6 / 17 / 97$ & 12694 & 60 & 88.2 & S97T001261 \\
\hline BX-110 & 197 & Salt & 2 & $6 / 17 / 97$ & 12744 & 60 & 100 & \$97T001267 \\
\hline \multirow[t]{2}{*}{ BX-110 } & 197 & Salt & $2 A$ & $6 / 17 / 97$ & 12741 & 60 & 19.4 & S97T001268 \\
\hline & & & & & & Total Weight & 278 & \\
\hline BX -110 & 198 & DL & 1 & $6 / 23 / 97$ & 12248 & 40 & 56.3 & S97T001318 \\
\hline \multirow[t]{2}{*}{ BX-110 } & 198 & DL & 2 & $6 / 23 / 97$ & 12249 & 40 & 16.2 & \$97T001319 \\
\hline & & & & & & Total Weight & 72.5 & \\
\hline BX-110 & 198 & FB & Fblk & $6 / 5 / 97$ & 12568 & 250 & 161.5 & S97T001313 \\
\hline \multirow[t]{2}{*}{ BX-110 } & 198 & FB & Fblk & $6 / 23 / 97$ & 12247 & 40 & 40.8 & S97T001317 \\
\hline & & & & & & Total Weight & 202.3 & \\
\hline BX-110 & 198 & Salt & 2 & $6 / 23 / 97$ & 12731 & 60 & 124.3 & $\mathrm{~S} 97 \mathrm{~T} 001301$ \\
\hline BX-110 & 198 & Salt & 2 & $6 / 23 / 97$ & 12732 & 60 & 68.2 & S97T001302 \\
\hline BX-110 & 198 & Salt & 2 & $12 / 9 / 97$ & 13867 & 40 & 15 & S97T001298 \\
\hline BX -110 & 198 & Salt & 3 & $6 / 23 / 97$ & 12733 & 60 & 119 & S97T001303 \\
\hline
\end{tabular}




\section{Vials in 11A1 and 11A2}

\begin{tabular}{|c|c|c|c|c|c|c|c|c|}
\hline Tank \# & Core\# & Matrix & Segment \# & Date & Jar/Vial \# & Vial Size (mLs) & Net Wt\# Found (gms) & Laboratory \# \\
\hline BX-110 & 198 & Salt & 3 & $12 / 8 / 97$ & 13634 & $\begin{array}{c}60 \\
\text { Total Weight }\end{array}$ & $\frac{90.4}{416.9}$ & S97T001300. \\
\hline $\begin{array}{l}\text { BX-110 } \\
\text { BX-110 }\end{array}$ & $\begin{array}{l}198 \\
198\end{array}$ & $\begin{array}{l}\text { Sludge } \\
\text { Sludge }\end{array}$ & $\begin{array}{l}4 \\
4\end{array}$ & $\begin{array}{r}6 / 5 / 97 \\
6 / 23 / 97\end{array}$ & $\begin{array}{l}12570 \\
12734\end{array}$ & $\begin{array}{c}250 \\
60 \\
\text { Total Weight }\end{array}$ & $\begin{array}{c}123.9 \\
62.8 \\
186.7\end{array}$ & $\begin{array}{l}\text { S97T001325 } \\
\text { S97T001327 }\end{array}$ \\
\hline BX-110 & R6 & Sludge & Aug-45 & $10 / 23 / 95$ & 8475 & $\begin{array}{l}60 \\
\text { Total Weight }\end{array}$ & $\frac{65.4}{65.4}$ & S95T002947 \\
\hline Tank \# & Core \# & Matrix & Segment \# & Date & Jar/Vial \# & Vial Size (mLs) & Net Wt\# Found (gms) & Laboratory \# \\
\hline BX-111 & 200 & $\mathrm{DL}$ & $2 A$ & $6 / 9 / 97$ & 12649 & $\begin{array}{c}60 \\
\text { Total Weight }\end{array}$ & $\begin{array}{r}17.8 \\
17.8\end{array}$ & S97T001375 \\
\hline $\begin{array}{l}\text { BX-111 } \\
\text { BX-111 }\end{array}$ & $\begin{array}{l}200 \\
200\end{array}$ & $\begin{array}{l}\text { FB } \\
\text { FB }\end{array}$ & $\begin{array}{l}\text { Folk } \\
\text { Folk }\end{array}$ & $\begin{array}{r}6 / 10 / 97 \\
7 / 1 / 97\end{array}$ & $\begin{array}{l}12571 \\
13212\end{array}$ & $\begin{array}{c}250 \\
40 \\
\text { Total Weight }\end{array}$ & $\begin{array}{l}196.5 \\
38.5 \\
235\end{array}$ & $\begin{array}{l}\text { S97T001376 } \\
\text { S97T001386 }\end{array}$ \\
\hline $\begin{array}{l}\text { BX-111 } \\
\text { BX-111 } \\
\text { BX-111 }\end{array}$ & $\begin{array}{l}200 \\
200 \\
200\end{array}$ & $\begin{array}{l}\text { Salt } \\
\text { Salt } \\
\text { Salt }\end{array}$ & $\begin{array}{c}1 \\
2 \\
2 \mathrm{~A}\end{array}$ & $\begin{array}{l}7 / 1 / 97 \\
7 / 1 / 97 \\
7 / 1 / 97\end{array}$ & $\begin{array}{l}13021 \\
12647 \\
13022\end{array}$ & $\begin{array}{c}60 \\
60 \\
60 \\
\text { Total Weight }\end{array}$ & $\begin{array}{c}67.1 \\
78.1 \\
94 \\
239.2\end{array}$ & $\begin{array}{l}\text { S97T001383 } \\
\text { S97T001384 } \\
\text { S97T001385 }\end{array}$ \\
\hline $\begin{array}{l}\text { BX-111 } \\
\text { BX-111 } \\
\text { BX-111 }\end{array}$ & $\begin{array}{l}202 \\
202 \\
202\end{array}$ & $\begin{array}{l}\text { Salt } \\
\text { Salt } \\
\text { Salt }\end{array}$ & $\begin{array}{l}1 \\
2 \\
3\end{array}$ & $\begin{array}{l}6 / 25 / 97 \\
6 / 27 / 97 \\
6 / 26 / 97\end{array}$ & $\begin{array}{l}13031 \\
13032 \\
13033 \\
\end{array}$ & $\begin{array}{c}60 \\
60 \\
60 \\
\text { Total Weight }\end{array}$ & $\begin{array}{l}59.3 \\
74.4 \\
41.3 \\
175\end{array}$ & $\begin{array}{l}\text { S97T001393 } \\
\text { S97T001394 } \\
\text { S97T001395 }\end{array}$ \\
\hline Tank\# & Core\# & Matrix & Segment \# & Date & Jar/Vial \# & Vial Size (mLs) & Net Wt\# Found (gms) & Laboratory \# \\
\hline $\begin{array}{l}\text { BX-112 } \\
\text { BX-112 }\end{array}$ & $\begin{array}{l}118 \\
118\end{array}$ & $\begin{array}{l}\text { Sludge } \\
\text { Sludge }\end{array}$ & $\begin{array}{l}1 \\
1\end{array}$ & $\begin{array}{l}1 / 22 / 96 \\
1 / 22 / 96\end{array}$ & $\begin{array}{l}9151 \\
9152\end{array}$ & $\begin{array}{c}60 \\
60 \\
\text { Total Weight }\end{array}$ & $\begin{array}{l}62.5 \\
72.5 \\
135\end{array}$ & $\begin{array}{l}\text { S96T000295 } \\
\text { S96T000297 }\end{array}$ \\
\hline BX-112 & R2 & Sludge & Aug-48 & $11 / 21 / 95$ & 8676 & $\begin{array}{l}60 \\
\text { Total Weight }\end{array}$ & $\begin{array}{r}41.3 \\
41.3\end{array}$ & S95T003756 \\
\hline
\end{tabular}




\section{Vials in 11A1 and 11A2}

\begin{tabular}{lllcccccc} 
Tank \# & Core \# & Matrix & Segment \# & Date & Jar/Vial \# & Vial Size (mLs) & Net Wt\# Found (gms) & Laboratory \# \\
\hline BX-112 & R3 & Sludge & Aug-47 & $11 / 21 / 95$ & 8195 & 125 & 43.9 & S95T003749 \\
BX-112 & R3 & Sludge & Aug-47 & $11 / 21 / 95$ & 8672 & 60 & 77 & S95T003752 \\
BX-112 & R3 & Sludge & Aug-47 & $11 / 21 / 95$ & 8673 & 60 & 7.8 & S95T003748.
\end{tabular}

Tank \# Core \# Matrix $\quad$ Segment \# $\quad$ Date $\quad$ Jar/Vial \# Vial Size (mLs) Net W\# Found (gms) Laboratory \#

\begin{tabular}{|c|c|c|c|c|c|c|c|c|}
\hline BY-101 & 189 & Sait & 2 & $6 / 6 / 97$ & 12695 & $\begin{array}{c}60 \\
\text { Total Weight }\end{array}$ & $\frac{31.2}{31.2}$ & S97T001356 \\
\hline $\begin{array}{l}\text { BY-101 } \\
\text { BY-101 }\end{array}$ & $\begin{array}{l}199 \\
199\end{array}$ & $\begin{array}{l}\text { FB } \\
\text { FB }\end{array}$ & $\begin{array}{l}\text { Folk } \\
\text { Folk }\end{array}$ & $\begin{array}{l}6 / 10 / 97 \\
7 / 17 / 97\end{array}$ & $\begin{array}{l}12573 \\
13289\end{array}$ & $\begin{array}{c}250 \\
40 \\
\text { Total Weight }\end{array}$ & $\begin{array}{c}170.1 \\
40.4 \\
210.5\end{array}$ & $\begin{array}{l}\text { S97T001367 } \\
\text { S97T001366 }\end{array}$ \\
\hline BY-101 & 199 & Salt & 1 & $6 / 6 / 97$ & 12696 & $\begin{array}{c}60 \\
\text { Total Weight }\end{array}$ & $\begin{array}{r}12.9 \\
12.9\end{array}$ & S97T001361 \\
\hline
\end{tabular}

\begin{tabular}{|c|c|c|c|c|c|c|c|c|}
\hline Tank \# & Core \# & Matrix & Segment \# & Date & Jar/Vial \# & Vial Size (mLs) & Net Wt\# Found (gms) & Laboratory \# \\
\hline$\overline{B Y}-102$ & 157 & $\mathrm{DL}$ & 7 & $7 / 29 / 96$ & 10777 & 60 & 50.5 & S96T004132 \\
\hline \multirow[t]{2}{*}{ BY -102} & 157 & $\mathrm{DL}$ & 8 & $7 / 21 / 96$ & 9938 & 40 & 55.9 & S96T003658 \\
\hline & & & & & & Total Weight & 106.4 & \\
\hline BY-102 & 157 & Salt & 1 & $8 / 8 / 96$ & 10851 & 60 & 27.8 & S96T004297 \\
\hline BY -102 & 157 & Salt & 2 & $7 / 21 / 96$ & 10668 & 60 & 85.3 & S96T003634 \\
\hline $\mathrm{BY}-102$ & 157. & Salt & 3 & $7 / 21 / 96$ & 10669 & 60 & 58.2 & S96T003647 \\
\hline BY-102 & 157 & Salt & 4 & $7 / 29 / 96$ & 10764 & 60 & 10.5 & S96T004124 \\
\hline BY -102 & 157 & Salt & 4 & $7 / 29 / 96$ & 10765 & 60 & 8 & S96T004125 \\
\hline BY-102 & 157 & Salt & 4 & $8 / 6 / 96$ & 10845 & 60 & 63.7 & S96T004157 \\
\hline BY -102 & 157 & Salt & 5 & $8 / 13 / 96$ & 10961 & 60 & 60.6 & S96T004342 \\
\hline $\mathrm{BY}-102$ & 157 & Salt & $5 A$ & $7 / 29 / 96$ & 10776 & 60 & 15 & S96T004126 \\
\hline BY -102 & 157 & Salt & SA & $8 / 6 / 96$ & 10846 & 60 & 35.9 & \$96T004158 \\
\hline BY -102 & 157 & Salt & 6 & $7 / 21 / 96$ & 10670 & 60 & 86.6 & S96T003648 \\
\hline BY-102 & 157 & Salt & 6 & $7 / 21 / 96$ & 10671 & 60 & 39.1 & S96T003649 \\
\hline BY-102 & 157 & Salt & 7 & $7 / 29 / 96$ & 10780 & 60 & 37.8 & S96T004128 \\
\hline BY-102 & 157 & Salt & 7 & $7 / 29 / 96$ & 10781 & 60 & 48.2 & S96T004129 \\
\hline
\end{tabular}


Vials in 11A1 and 11A2

\begin{tabular}{|c|c|c|c|c|c|c|c|c|}
\hline Tank\# & Core \# & Matrix & Segment \# & Date & Jar/Vial \# & Vial Size (mLs) & Net WH Found (gms) & Laboratory \# \\
\hline BY-102 & 157 & Salt & 7 & $7 / 29 / 96$ & 10782 & 60 & 20.7 & S96T004130 \\
\hline BY-102 & 157 & Salt & 7 & $8 / 6 / 96$ & 10847 & $\begin{array}{l}\quad 60 \\
\text { Total Weight }\end{array}$ & $\begin{array}{l}73.6 \\
671\end{array}$ & S96T004159 \\
\hline BY-102 & 159 & FB & Folk & $8 / 8 / 96$ & 10569 & 250 & 133.3 & S96T004299 \\
\hline$B Y-102$ & 159 & $\mathrm{FB}$ & Folk & $8 / 8 / 96$ & 10868 & $\begin{array}{l}40 \\
\text { Total Weight }\end{array}$ & $\frac{32.9}{166.2}$ & S96T004301 \\
\hline
\end{tabular}

\begin{tabular}{|c|c|c|c|c|c|c|c|c|}
\hline Tank \# & Core\# & Matrix & Segment \# & Date & Jar/Vial \# & Vial Size (mLs) & Net Wt\# Found (gms) & Laboratory \# \\
\hline BY-103 & Grab & GL & 1 & 9/2/97 & 13239 & 40 & 31.3 & S95T000493 \\
\hline BY-103 & Grab & GL & 2 & $9 / 3 / 97$ & 13238 & 40 & 30.7 & S95T000495 \\
\hline & & & & & & Total Weight & 62 & \\
\hline BY-103 & R10B & Sludge & Aug-12 & $3 / 30 / 95$ & 6756 & $\begin{array}{l}40 \\
\text { Total Weight }\end{array}$ & $\frac{21.9}{21.9}$ & S95T000509 \\
\hline BY-103 & R12A & Sludge & Aug-13 & $3 / 30 / 95$ & 6877 & $\begin{array}{l}40 \\
\text { Total Weight }\end{array}$ & $\frac{24.6}{24.6}$ & S95T000513 \\
\hline
\end{tabular}

\begin{tabular}{|c|c|c|c|c|c|c|c|c|}
\hline Tank\# & Core \# & Matrix & Segment \# & Date & Jar/Vial \# & Vial Size (mLs) & Net Wt\# Found (gms) & Laboratory \# \\
\hline BY-104 & 116 & FB & Folk & $11 / 14 / 95$ & 8397 & $\begin{array}{l}40 \\
\text { Total Weight }\end{array}$ & $\begin{array}{l}5.4 \\
5.4\end{array}$ & S95T003714 \\
\hline BY-104 & 116 & Salt & 2 & $11 / 29 / 95$ & 8410 & 60 & 20.6 & S95T003526 \\
\hline BY-104 & 116 & Salt & 2 & $11 / 30 / 95$ & 8411 & 40 & 69.7 & S95T003527 \\
\hline BY-104 & 116 & Salt & 3 & $11 / 7 / 95$ & 8471 & 250 & 46.6 & S95T003611 \\
\hline BY-104 & 116 & Salt & 3 & $11 / 28 / 95$ & 8757 & 60 & 29.4 & S95T003631 \\
\hline BY-104 & 116 & Salt & 3 & $11 / 29 / 95$ & 8758 & 60 & 70.1 & S95T003630 \\
\hline BY-104 & 116 & Salt & 4 & $11 / 29 / 95$ & 8759 & 60 & 50.3 & S9ST003613 \\
\hline BY-104 & 116 & Salt & 4 & $11 / 29 / 95$ & 8760 & 60 & 41.3 & S95T003614 \\
\hline BY-104 & 116 & Salt & 4 & $11 / 29 / 95$ & 8761 & 60 & 46.1 & S95T003615 \\
\hline BY-104 & 116 & Salt & 5 & $12 / 1 / 95$ & 8762 & 60 & 82.5 & \$95T003616 \\
\hline BY-104 & 116 & Salt & 5 & $12 / 1 / 95$ & 8763 & 60 & 66.8 & S95T003617 \\
\hline BY-104 & 116 & Salt & 5 & $12 / 4 / 95$ & 8764 & 60 & 85.1 & S95T003618 \\
\hline BY-104 & 116 & Salt & 5 & $12 / 5 / 95$ & 8765 & 60 & 65.6 & S95T003619 \\
\hline 17-Mar- & & & & & & & & ge 47 of 114 \\
\hline
\end{tabular}


Vials in 11A1 and 11A2

\begin{tabular}{|c|c|c|c|c|c|c|c|c|c|}
\hline Tank \# & Core \# & Matrix & Segment \# & Date & Jar/Vial \# & Vial Size (mLs) & \multicolumn{2}{|c|}{ Net Wt\# Found (gms) } & Laboratory \# \\
\hline BY-104 & 116 & Salt & 6 & $11 / 10 / 95$ & 8779 & 60 & \multicolumn{2}{|l|}{84.1} & S95T003622 \\
\hline BY-104 & 116 & Salt & 6 & $12 / 5 / 95$ & 8777 & 60 & \multicolumn{2}{|l|}{67.5} & S95T003620 \\
\hline BY-104 & 116 & Salt & 6 & $12 / 5 / 95$ & 8780 & 60 & \multicolumn{2}{|l|}{78.3} & S95T003604 \\
\hline BY-104 & 116 & Salt & 6 & $12 / 5 / 96$ & 8778 & 60 & \multicolumn{2}{|l|}{62,7} & S95T003621 \\
\hline BY -104 & 116 & Salt & 7 & $11 / 10 / 95$ & 8769 & 60 & \multicolumn{2}{|l|}{85.7} & S95T003624 \\
\hline BY-104 & 116 & Salt & 7 & $11 / 10 / 95$ & 8771 & 60 & \multicolumn{2}{|l|}{85} & S9ST003626 \\
\hline BY-104 & 116 & Salt & 7 & $11 / 10 / 95$ & 8772 & 60 & \multicolumn{2}{|l|}{77.9} & S95T003627 \\
\hline BY-104 & 116 & Salt & 7 & $12 / 5 / 96$ & 8770 & 60 & \multicolumn{2}{|l|}{68.6} & S95T003625 \\
\hline & & & & & & Total Weight & \multicolumn{2}{|l|}{$\overline{1283.9}$} & \\
\hline BY-104 & 116 & Sludge & 8 & $11 / 10 / 95$ & 8782 & 60 & \multicolumn{2}{|l|}{77.6} & S95T003628 \\
\hline BY-104 & 116 & Sludge & 8 & $12 / 5 / 95$ & 8784 & 60 & \multicolumn{2}{|l|}{86.3} & S95T003629 \\
\hline & & & & & & Total Weight & \multicolumn{2}{|l|}{163.9} & \\
\hline BY-104 & 117 & DL & 1 & $11 / 21 / 95$ & 8473 & 250 & \multicolumn{2}{|l|}{80.7} & S95T003760 \\
\hline BY -104 & 117 & DL & 1 & $12 / 13 / 95$ & 8412 & 40 & \multicolumn{2}{|l|}{60.9} & S95T003762 \\
\hline BY-104 & 117 & $\mathrm{DL}$ & 2 & $12 / 14 / 95$ & 8805 & 60 & \multicolumn{2}{|l|}{63.7} & S95T003808 \\
\hline & & & & & & Total Weight & \multicolumn{2}{|l|}{205.3} & - \\
\hline BY-104 & 117 & Salt & 1 & $12 / 13 / 95$ & 8791 & 60 & \multicolumn{2}{|l|}{7.8} & \$95T003766 \\
\hline BY-104 & 117 & Salt & 3 & $12 / 15 / 95$ & 8802 & 60 & \multicolumn{2}{|l|}{62.2} & S95T003804 \\
\hline BY -104 & 117 & Salt & 3 & $12 / 15 / 95$ & 8806 & 60 & \multicolumn{2}{|l|}{127.6} & S95T003803 \\
\hline BY-104 & 117 & Salt & 4 & $12 / 13 / 95$ & 8807 & 60 & \multicolumn{2}{|l|}{128} & S95T003805 \\
\hline & & & & & & Total Weight & \multicolumn{2}{|l|}{325.6} & 200 \\
\hline BY-104 & 117 & Sludge & 2 & $12 / 14 / 95$ & 8803 & 60 & \multicolumn{2}{|l|}{14 : } & S95T003801 \\
\hline BY-104 & 117 & Sludge & 2 & $12 / 14 / 95$ & 8804 & 60 & \multicolumn{2}{|l|}{113.4} & S95T003802 \\
\hline BY -104 & 117 & Sludge & 5 & $12 / 15 / 95$ & 8808 & 60 & \multicolumn{2}{|l|}{24.3} & S95T003806 \\
\hline BY-104 & 117 & Sludge & 5 & $12 / 15 / 95$ & 8810 & 60 & \multicolumn{2}{|l|}{20.8} & S95T003807 \\
\hline & & & & & & Total Weight & \multicolumn{2}{|l|}{172.5} & \\
\hline
\end{tabular}

\begin{tabular}{lllcccccc} 
Tank \# & Core \# & Matrix & Segment \# & Date & Jar/Vial \# & Vial Size (mLs) & Net Wt\# Found (gms) & Laboratory \# \\
\hline BY-105 & 108 & DL & 1 & $11 / 10 / 95$ & 8394 & 40 & 26.5 & S95T003387 \\
BY-105 & 108 & DL & 1 & $11 / 10 / 95$ & 8395 & 40 & 42.5 & S95T003387. \\
BY-105 & 108 & DL & 1AR & $11 / 13 / 95$ & 8398 & 40 & 50.8 & S95T003413 \\
BY-105 & 108 & DL & 1 AR & $11 / 13 / 95$ & 8399 & 40 & S95T003413
\end{tabular}


Vials in 11A1 and 11A2

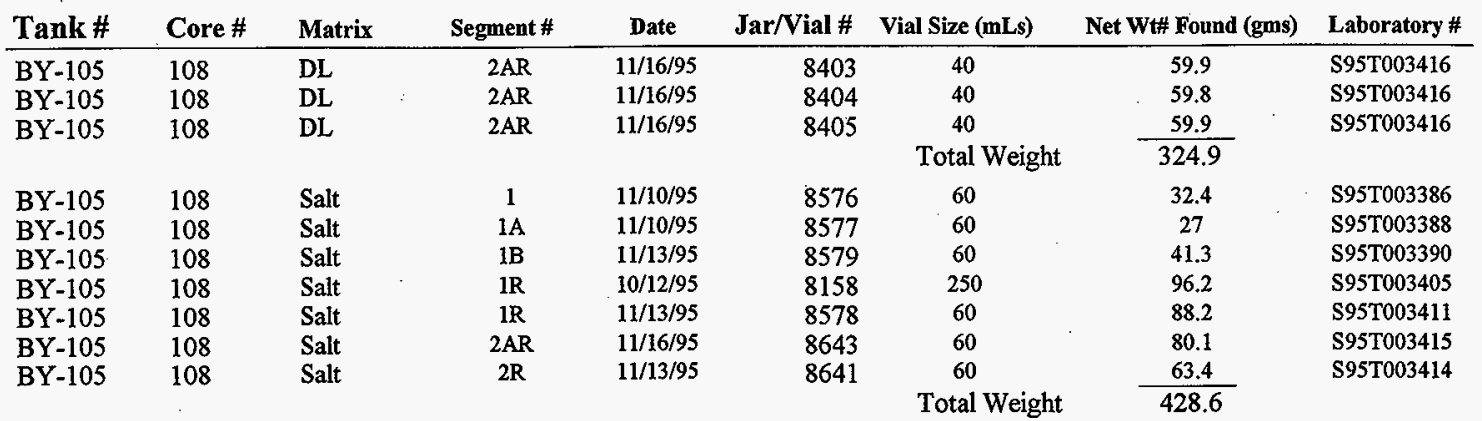

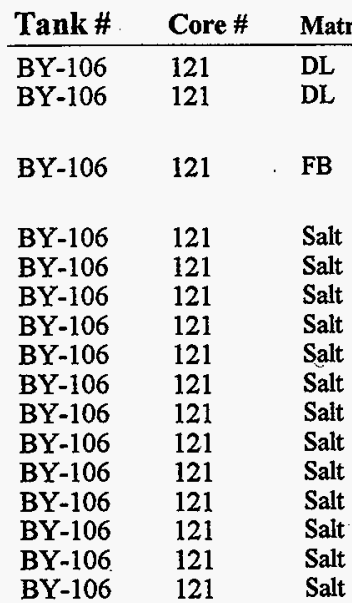

\begin{tabular}{|c|c|c|c|c|c|}
\hline Segment \# & Date & Jar/Vial \# & Vial Size (mLs) & Net Wt\# Found (gms) & Laboratory \# \\
\hline $10 \mathrm{~B}$ & $1 / 16 / 96$ & 8858 & 250 & 161.9 & S96T000257 \\
\hline $10 \mathrm{~B}$ & $1 / 21 / 96$ & 8422 & $\begin{array}{l}40 \\
\text { Total Weight }\end{array}$ & $\frac{56.7}{218.6}$ & S96T000274 \\
\hline Fblk & $1 / 16 / 96$ & 8857 & $\begin{array}{l}250 \\
\text { Total Weight }\end{array}$ & $\frac{149.9}{149.9}$ & S95T000278 \\
\hline 10 & $1 / 19 / 96$ & 9148 & 60 & 73.6 & S96T000103 \\
\hline $10 \mathrm{~B}$ & $1 / 21 / 96$ & 9149 & 60 & 84.2 & S96T000247 \\
\hline $10 \mathrm{~B}$ & $1 / 21 / 96$ & 9150 & 60 & 19.5 & S96T000246 \\
\hline 2 & $1 / 12 / 96$ & 8980 & 60 & 19.6 & S95T004156 \\
\hline 3. & $1 / 12 / 96$ & 9003 & 60 & 96.4 & S95T004157 \\
\hline 3 & $1 / 14 / 96$ & 11820 & 60 & 17.2 & S95T004158 \\
\hline 4 & $1 / 12 / 96$ & 9004 & 60 & 94.9 & S95T004159 \\
\hline 4 & $1 / 12 / 96$ & 9005 & 60 & 81.8 & S95T004160 \\
\hline 5 & $1 / 12 / 96$ & 9006 & 60 & 97.4 & S95T004161 \\
\hline 6 & $1 / 12 / 96$ & 9007 & 60 & 92.6 & S95T004162 \\
\hline 7 & $1 / 14 / 96$ & 9022 & 60 & 90.5 & S95T004163 \\
\hline 8 & $1 / 14 / 96$ & 9023 & 60 & 66.2 & S95T004164 \\
\hline 8 & $1 / 14 / 96$ & 9024 & 60 & 51.2 & S95T004165 \\
\hline
\end{tabular}


Vials in 11A1 and 11A2

\begin{tabular}{|c|c|c|c|c|c|c|c|c|}
\hline Tank\# & Core \# & Matrix & Segment \# & Date & Jar/Vial \# & Vial Size (mLs) & Net Wt\# Found (gms) & Laboratory \# \\
\hline $\begin{array}{l}\text { BY-106 } \\
\text { BY-106 }\end{array}$ & $\begin{array}{l}121 \\
121\end{array}$ & $\begin{array}{l}\text { Salt } \\
\text { Salt }\end{array}$ & $\stackrel{9}{\text { Comp }}$ & $\begin{array}{r}1 / 14 / 96 \\
2 / 6 / 96\end{array}$ & $\begin{array}{l}8414 \\
8604\end{array}$ & $\begin{array}{c}40 \\
125 \\
\text { Total Weight }\end{array}$ & $\begin{array}{c}7.5 \\
143.2 \\
1035.8\end{array}$ & $\begin{array}{l}\text { S95T004166 } \\
\text { S96T000376 }\end{array}$ \\
\hline $\begin{array}{l}\text { BY-106 } \\
\text { BY-106 } \\
\text { BY-106 } \\
\text { BY-106 }\end{array}$ & $\begin{array}{l}64 \\
64 \\
64 \\
64\end{array}$ & $\begin{array}{l}\mathrm{DL} \\
\mathrm{DL} \\
\mathrm{DL} \\
\mathrm{DL}\end{array}$ & $\begin{array}{c}10 \\
10 \\
3 \\
3\end{array}$ & $\begin{array}{r}12 / 22 / 94 \\
12 / 22 / 94 \\
12 / 1 / 94 \\
12 / 6 / 94\end{array}$ & $\begin{array}{l}6136 \\
7045 \\
6134 \\
7032\end{array}$ & $\begin{array}{c}250 \\
40 \\
250 \\
40 \\
\text { Total Weight }\end{array}$ & $\begin{array}{c}188.1 \\
36.2 \\
42.3 \\
45.3 \\
311.9\end{array}$ & \\
\hline BY-106 & 64 & FB & Fblk & $12 / 27 / 94$ & 6137 & $\begin{array}{l}250 \\
\text { Total Weight }\end{array}$ & $\begin{array}{r}138.4 \\
138.4\end{array}$ & \\
\hline $\begin{array}{l}\text { BY-106 } \\
\text { BY-106 } \\
\text { BY-106 } \\
\text { BY-106 } \\
\text { BY-106 } \\
\text { BY-106 } \\
\text { BY-106 } \\
\text { BY-106 } \\
\text { BY-106 } \\
\text { BY-106 } \\
\text { BY-106 } \\
\text { BY-106 } \\
\text { BY-106 } \\
\text { BY-106 } \\
\text { BY-106 } \\
\text { BY-106 }\end{array}$ & $\begin{array}{l}64 \\
64 \\
64 \\
64 \\
64 \\
64 \\
64 \\
64 \\
64 . \\
64 \\
64 \\
64 \\
64 \\
64 \\
64 \\
64\end{array}$ & $\begin{array}{l}\text { Salt } \\
\text { Salt } \\
\text { Salt } \\
\text { Salt } \\
\text { Salt } \\
\text { Salt } \\
\text { Salt } \\
\text { Salt } \\
\text { Salt } \\
\text { Salt } \\
\text { Salt } \\
\text { Salt } \\
\text { Salt } \\
\text { Salt } \\
\text { Salt } \\
\text { Salt }\end{array}$ & $\begin{array}{c}10 \\
10 \\
11 \\
13 \\
4 \\
4 \\
4 \\
4 \\
8 \\
8 \\
8 \\
8 \\
8 \\
8 \\
9 \\
\text { Comp }\end{array}$ & $\begin{array}{r}12 / 22 / 94 \\
12 / 22 / 94 \\
12 / 27 / 94 \\
12 / 28 / 94 \\
12 / 2 / 94 \\
12 / 2 / 94 \\
12 / 6 / 94 \\
12 / 6 / 94 \\
12 / 8 / 94 \\
12 / 8 / 94 \\
12 / 8 / 94 \\
12 / 8 / 94 \\
12 / 8 / 94 \\
12 / 8 / 94 \\
12 / 19 / 94 \\
9 / 22 / 97\end{array}$ & $\begin{array}{r}7046 \\
7047 \\
7042 \\
7048 \\
6160 \\
6161 \\
7030 \\
7031 \\
6352 \\
6353 \\
6354 \\
7027 \\
7040 \\
7043 \\
7041 \\
13457\end{array}$ & $\begin{array}{c}40 \\
40 \\
40 \\
40 \\
125 \\
125 \\
40 \\
40 \\
125 \\
125 \\
125 \\
40 \\
40 \\
40 \\
40 \\
60 \\
\text { Total Weight }\end{array}$ & $\begin{array}{c}15.6 \\
38.3 \\
34.88 \\
5.01 \\
73.6 \\
146.1 \\
41.8 \\
33.5 \\
59.2 \\
55.1 \\
104.1 \\
41.9 \\
48 \\
43.2 \\
52.1 \\
82.3 \\
874.69\end{array}$ & \\
\hline $\begin{array}{l}\text { BY-106 } \\
\text { BY-106 } \\
\text { BY-106 } \\
\text { BY-106 }\end{array}$ & $\begin{array}{l}64 \\
64 \\
64 \\
64\end{array}$ & $\begin{array}{l}\text { Sludge } \\
\text { Sludge } \\
\text { Sludge } \\
\text { Sludge }\end{array}$ & $\begin{array}{c}12 \\
2 \\
2 \\
3\end{array}$ & $\begin{array}{r}12 / 28 / 94 \\
11 / 30 / 94 \\
12 / 6 / 94 \\
12 / 1 / 94\end{array}$ & $\begin{array}{l}6381 \\
6350 \\
7033 \\
7026\end{array}$ & $\begin{array}{c}40 \\
125 \\
40 \\
40 \\
\text { Total Weight }\end{array}$ & $\begin{array}{c}65.7 \\
80.5 \\
32.5 \\
24.4 \\
203.1\end{array}$ & \\
\hline
\end{tabular}


Vials in 11A1 and 11A2

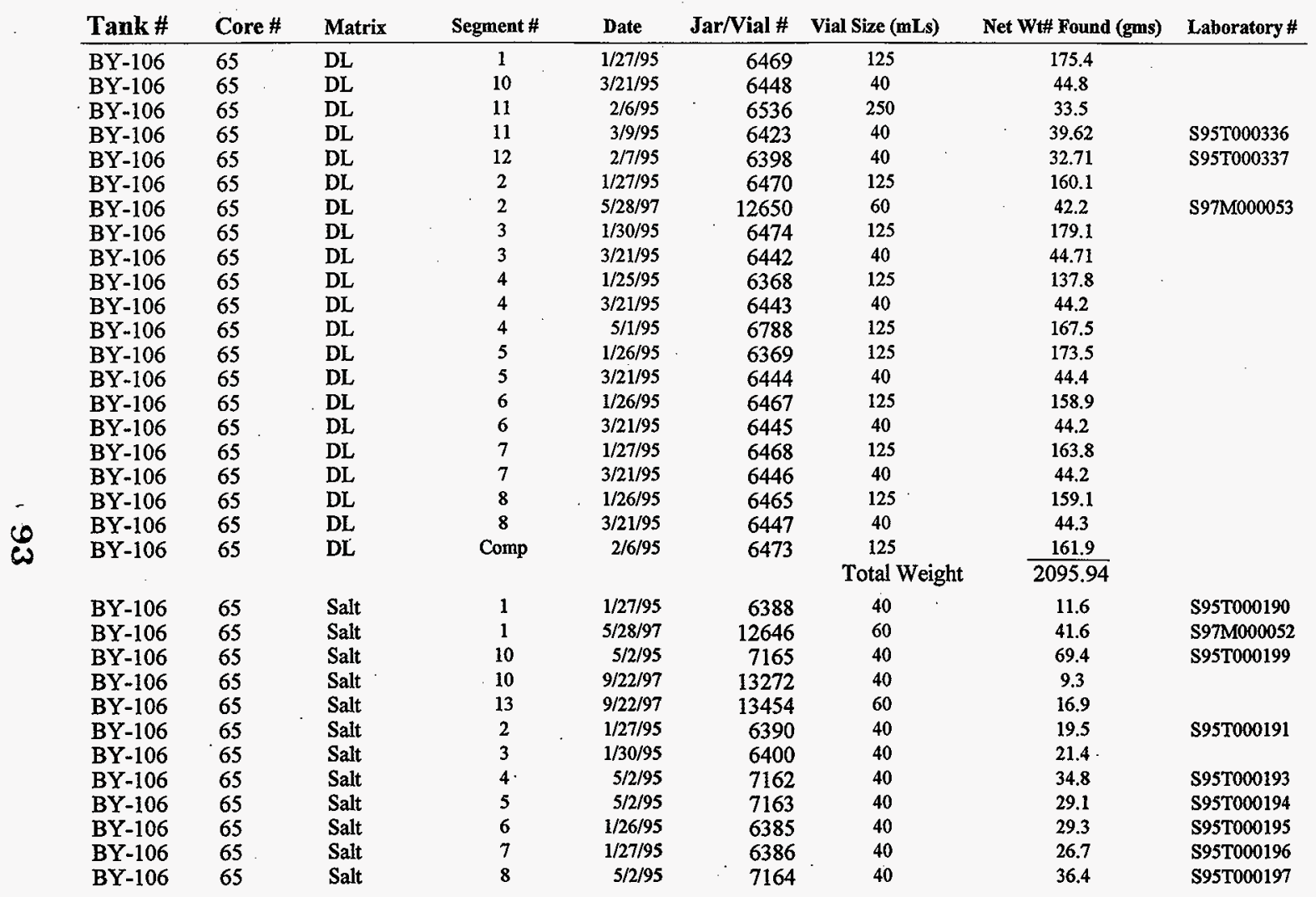


Vials in 11A1 and 11A2

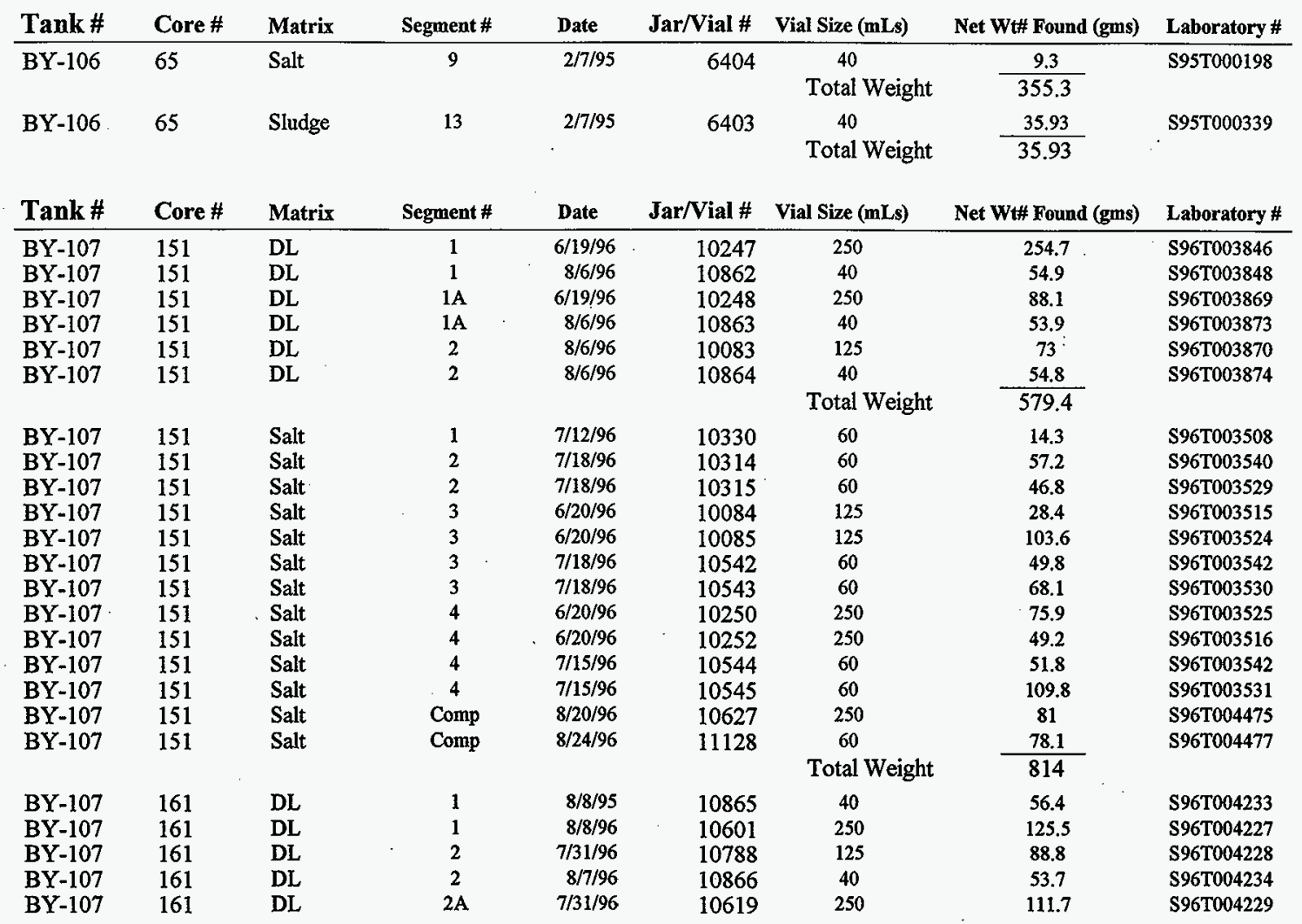


Vials in 11A1 and 11A2

\begin{tabular}{|c|c|c|c|c|c|c|c|c|}
\hline Tank \# & Core \# & Matrix & Segment \# & Date & Jar/Vial \# & Vial Size (mLs) & Net Wt\# Found (gms) & Laboratory \# \\
\hline BY-107 & 161. & DL & $2 \mathrm{~A}$ & $8 / 8 / 96$ & 10867 & 40 & 50.2 & S96T004235 \\
\hline BY-107 & 161 & DL & 3 & $8 / 12 / 96$ & 10954 & 60 & 50.2 & S96T004407 \\
\hline BY-107 & 161 & $\mathrm{DL}$ & $3 \mathrm{~A}$ & $8 / 13 / 96$ & 10958 & 60 & 90.5 & S96T004347 \\
\hline BY -107 & 161 & $\overline{\mathrm{DL}}$ & $3 \mathrm{~A}$ & $8 / 13 / 96$ & 10959 & 60 & 35.4 & S96T004350 \\
\hline \multirow[t]{2}{*}{ BY-107 } & 161 & DL & 4 & $8 / 12 / 96$ & 10956 & 60 & 48.3 & S96T004408 \\
\hline & & & & & & Total Weight & 710.7 & \\
\hline BY-107 & 161 & Salt & 1 & $8 / 8 / 96$ & 10848 & 60 & 30.8 & S96T004224 \\
\hline BY-107 & 161 & Salt & 2 & $7 / 31 / 96$ & 10771 & 60 & 10.6 & S96T004214 \\
\hline BY- 107 & 161 & Salt & 2 & $7 / 31 / 96$ & 10772 & 60 & 21.1 & S96T004215 \\
\hline BY -107 & 161 & Salt & 2 & $7 / 31 / 96$ & 10773 & 60 & 12.4 & S96T004213 \\
\hline BY-107 & 161 & Salt & $2 \mathrm{~A}$ & & 10850 & 60 & 27 & S96T004226 \\
\hline BY -107 & 161 & Salt & 3 & $8 / 12 / 96$ & 10955 & 60 & 52.8 & S96T004391 \\
\hline BY-107 & 161 & Salt & 4 & $8 / 12 / 96$ & 10957 & 60 & 42.2 & S96T004392 \\
\hline \multirow{2}{*}{ BY-107 } & 161 & Salt & Comp. & $8 / 23 / 96$ & 11127 & 60 & 55.7 & S96T004482 \\
\hline & & & & & & Total Weight & 252.6 & \\
\hline BY-107 & 161 & Sludge & 4 & $8 / 12 / 96$ & 10599 & 250 & 104.8 & S96T004393 \\
\hline \multirow[t]{2}{*}{ BY-107 } & 161 . & Sludge & 4 & $8 / 14 / 96$ & 10962 & 60 & 99.9 & S96T004394 \\
\hline & & & & & & Total Weight & 204.7 & \\
\hline
\end{tabular}

\begin{tabular}{|c|c|c|c|c|c|c|c|c|c|}
\hline \multirow{2}{*}{ ज } & \multirow{2}{*}{$\frac{\text { Tank \# }}{\text { BY-108 }}$} & \multirow{2}{*}{ Core \# } & \multirow{2}{*}{$\frac{\text { Matrix }}{\text { Salt }}$} & \multirow{2}{*}{$\begin{array}{c}\text { Segment \# } \\
\text { Aug-17 }\end{array}$} & \multirow{2}{*}{$\begin{array}{l}\text { Date } \\
9 / 24 / 97\end{array}$} & $\frac{\text { Jar/Vial \# }}{13460}$ & $\frac{\text { Vial Size (mLs) }}{60}$ & \multirow{2}{*}{$\begin{array}{c}\text { Net Wt\# Found (gms) } \\
23.7 \\
\end{array}$} & \multirow{2}{*}{ Laboratory } \\
\hline & & & & & & & $\begin{array}{c}60 \\
\text { Total Weig }\end{array}$ & & \\
\hline
\end{tabular}

$\begin{array}{llllllccc}\text { BY-108 } & 104 & \text { Salt } & 1 & 8 / 23 / 95 & 7496 & 250 & 69.8 & \text { S95T001920 } \\ \text { BY-108 } & 104 & \text { Salt } & 1 & 8 / 29 / 95 & 7656 & 40 & 35.1 & \text { S95T001926 } \\ \text { BY-108 } & 104 & \text { Salt } & 2 & 8 / 23 / 95 & 7497 & 250 & 43 & \text { S95T001934 } \\ \text { BY-108 } & 104 & \text { Salt } & 2 & 8 / 23 / 95 & 7521 & 125 & 45.3 & \text { S95T001935 } \\ \text { BY-108 } & 104 & \text { Salt } & 2 & 9 / 18 / 95 & 7679 & 40 & 60.3 & \text { S95T001984 } \\ \text { BY-108 } & 104 & \text { Salt } & 2 & 9 / 18 / 95 & 7686 & 40 & 49.6 & \text { S95T001985 } \\ \text { BY-108 } & 104 & \text { Salt } & 3 & 9 / 18 / 95 & 7687 & 40 & 58.9 & \text { S95T001986 } \\ \text { BY-108 } & 104 & \text { Salt } & 3 & 9 / 18 / 95 & 7688 & 40 & 72.7 & \text { S95T001987 } \\ \text { BY-108 } & 104 & \text { Salt } & 3 & 9 / 18 / 95 & 7689 & 40 & 45.9 & \text { S95T001988 } \\ \text { BY-108 } & 104 & \text { Salt } & 4 & 9 / 19 / 95 & 7691 & 40 & 67.2 & \text { S95T001990 }\end{array}$


Vials in 11A1 and 11A2

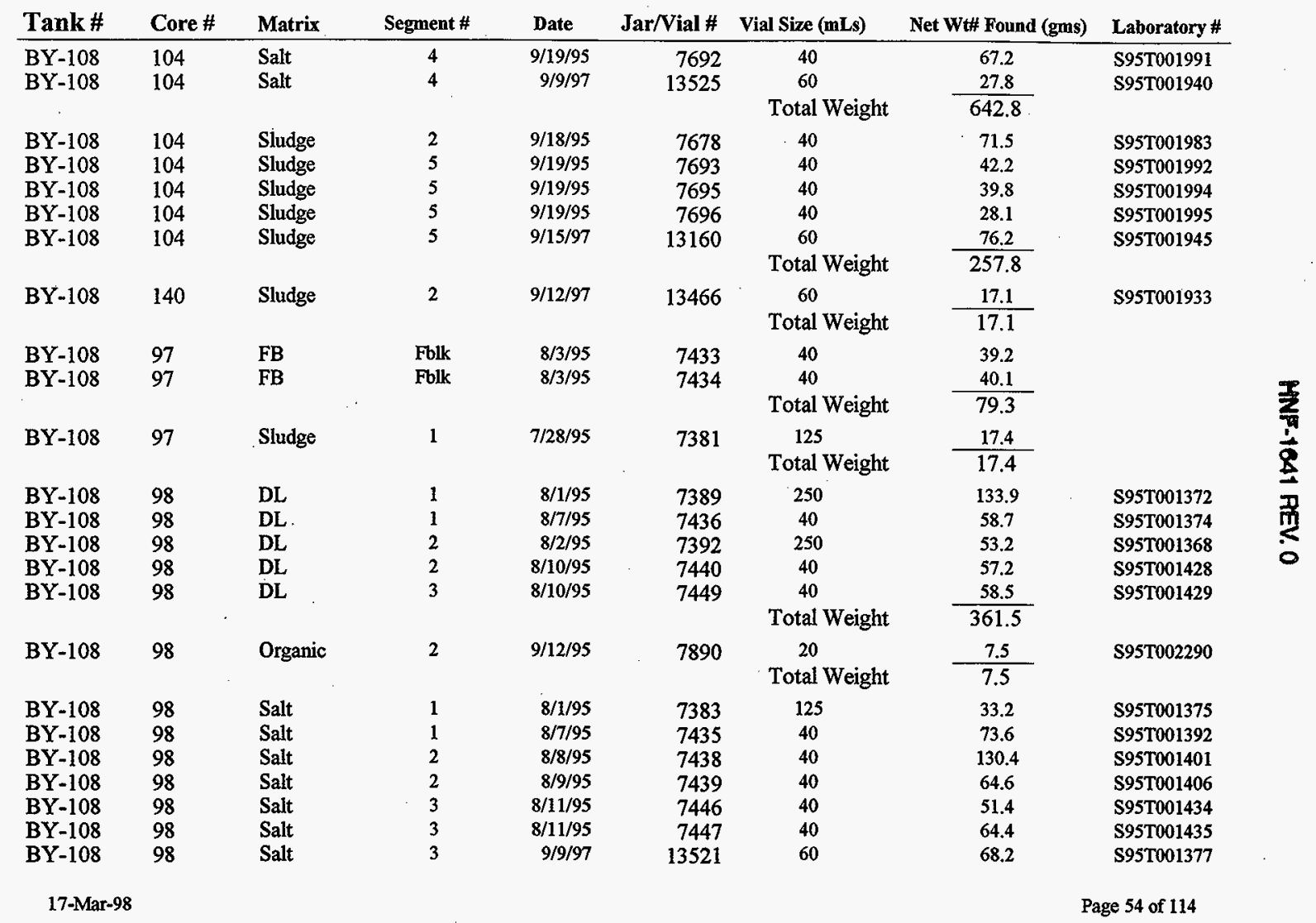


Vials in 11A1 and 11A2

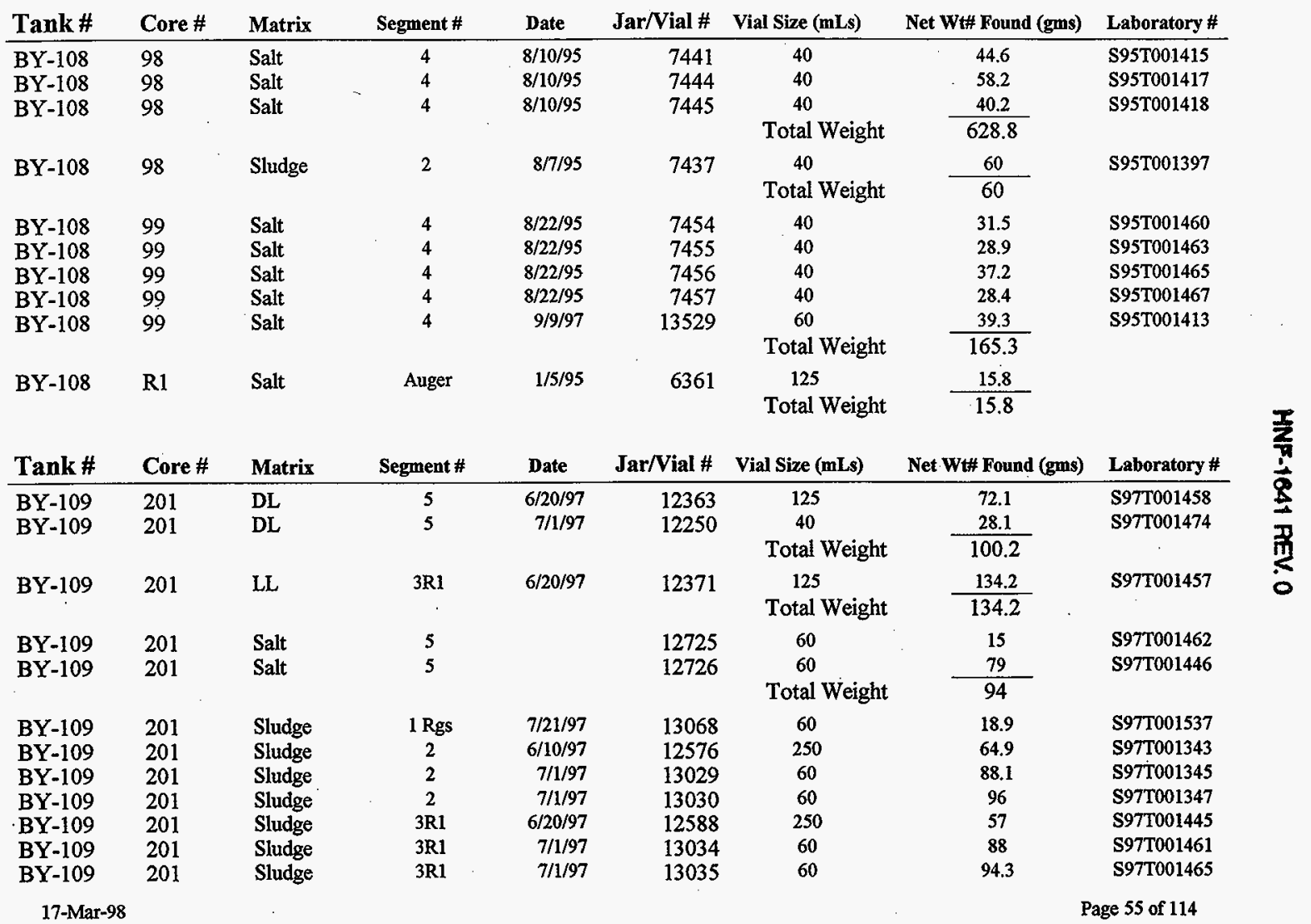


Vials in 11A1 and 11A2

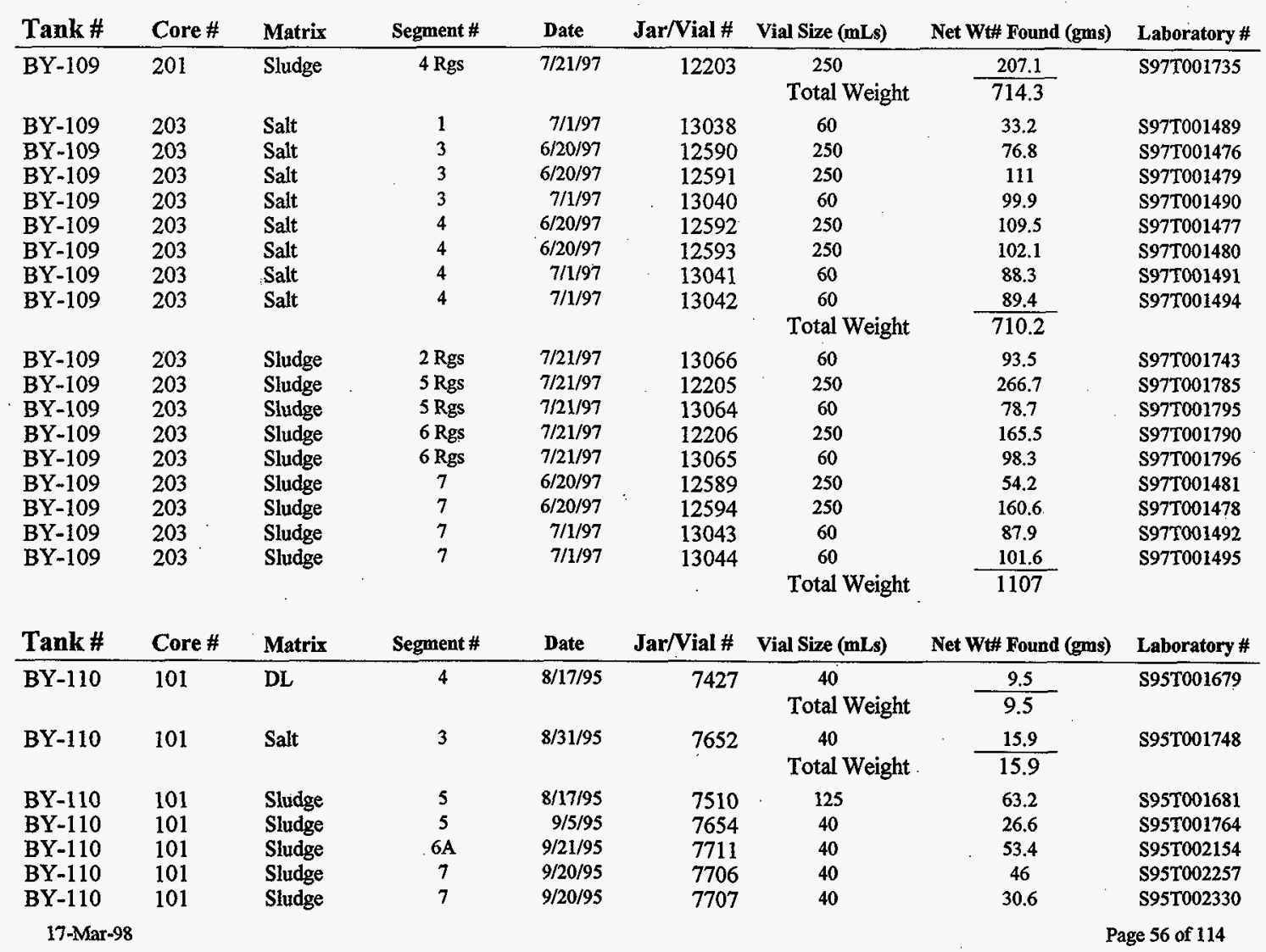


Vials in 11A1 and 11A2

\begin{tabular}{|c|c|c|c|c|c|c|c|c|}
\hline Tank \# & Core \# & Matrix & Segment \# & Date & Jar/Vial \# & Vial Size (mLs) & Net Wt\# Found (gms) & Laboratory \# \\
\hline BY-110 & 101 & Sludge & 7 & $9 / 20 / 95$ & 7708 & 40 & 41.1 & S95T002259 \\
\hline$B Y=110$ & 101 & Sludge & 7 & $9 / 20 / 95$ & 7709 & 40 & 23.6 & S95T002260 \\
\hline BY-110 & 101 & Sludge & 7 & 9/20/95 & 7710 & 40 & 71.9 & S95T002261 \\
\hline BY -110 & 101 & Sludge & 8 & $9 / 26 / 95$ & 7965 & 40 & 62.2 & S95T002262 \\
\hline$B Y-110$ & 101 & Sludge & 8 & $9 / 26 / 95$ & 7966 & 40 & 30.1 & S95T002265 \\
\hline BY -110 & 101 & Sludge & 8 & $9 / 26 / 95$ & 7967 & 40 & 47.9 & S95T002264 \\
\hline BY -110 & 101 & Sludge & 8 & $9 / 26 / 95$ & 7968 & 40 & 41.7 & S95T002263 \\
\hline BY -110 & 101 & Sludge & 9 & $9 / 26 / 95$ & 7964 & 40 & 69.4 & S95T002266 \\
\hline BY -110 & 101 & Sludge & 9 & $9 / 27 / 95$ & 7969 & 40 & 58.9 & S95T002267 \\
\hline BY -110 & 101 & Sludge & 9 & $9 / 27 / 95$ & 7970 & 40 & 46.9 & S95T002268 \\
\hline BY -110 & 101 & Sludge & 9 & $9 / 27 / 95$ & 7971 . & 40 & 68.1 & S95T002269 \\
\hline \multirow[t]{2}{*}{ BY -110} & 101 & Sludge & Comp & $1 / 20 / 96$ & 9206 & 60 & 15.9 & S96T000402 \\
\hline & & & & & & Total Weight & 797.5 & \\
\hline \multirow[t]{2}{*}{ BY-110 } & 102 & Sludge. & 1 & $9 / 23 / 97$ & 13458 & 60 & 55.8 & \\
\hline & & & & & & Total Weight & 55.8 & \\
\hline$B Y-110$ & 103 & DL & 7 & $8 / 23 / 95$ & 7513 & 125 & 70.8 & S95T001602 \\
\hline \multirow[t]{2}{*}{ BY -110} & 103 & DL & 7 & $8 / 25 / 95$ & 7472 & 40 & 64 & S95T001709 \\
\hline & & & & & & Total Weight & 134.8 & \\
\hline BY -110 & 103 & Salt & 1 & $8 / 21 / 95$ & 7511 & 40 & 11.6 & S95T001570 \\
\hline$B Y-110$ & 103 & Salt & 1 & $8 / 25 / 95$ & 7459 & 40 & 14.8 & S95T001652 \\
\hline$B Y-110$ & 103 & Salt & 1 & $8 / 25 / 95$ & 7460 & 40 & 12.1 & S95T001623 \\
\hline BY -110 & 103 & Salt & 1 & $9 / 12 / 97$ & 13472 & 60 & 53.8 & S95T001571 \\
\hline BY -110 & 103 & Salt & 1 & $9 / 12 / 97$ & $7485 \mathrm{~A}$ & 40 & 8.5 & 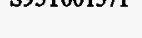 \\
\hline BY -110 & 103 & Salt & 2 & $8 / 25 / 95$ & $\begin{array}{r}7403 A \\
7461\end{array}$ & 40 & 34 & S95T001653 \\
\hline BY -110 & 103 & Salt & 3 & $8 / 25 / 95$ & 7463 & 40 & 29.1 & S95T001654 \\
\hline BY -110 & 103 & Salt & 4 & $8 / 25 / 95$ & 7464 & 40 & 27.9 & S95T001655 \\
\hline BY -110 & 103 & Salt & 4 & $1 / 29 / 96$ & 9168 & 60 & 11.3 & S95T001656 \\
\hline \multirow[t]{2}{*}{$B Y-110$} & 103 & Salt & Comp & $1 / 20 / 96$ & 9207 & 60 & 10.5 & S96T000403 \\
\hline & & & & & & Total Weight & 213.6 & \\
\hline$B Y-110$ & 103 & Sludge & 3 & $9 / 15 / 97$ & .13474 & 60 & 36.8 & S95T001581 \\
\hline$B Y-110$ & 103 & Sludge & 5 & $8 / 25 / 95$ & 7466 & 40 & 30.2 & S95T001657 \\
\hline BY-110 & 103 & Sludge & 6 & $8 / 25 / 95$ & 7468 & 40 & 65.9 & S95T001658 \\
\hline
\end{tabular}


Vials in 11A1 and 11A2

\begin{tabular}{|c|c|c|c|c|c|c|c|c|}
\hline Tank \# & Core\# & Matrix & Segment \# & Date & Jar/Vial \# & Vial Size (mLs) & Net Wt\# Found (gms) & Laboratory \# \\
\hline BY -110 & 103 & Sludge & 6 & $8 / 25 / 95$ & 7469 & 40 & 59.4 & S95T001706 \\
\hline BY -110 & 103 & Sludge & 6 & 9/9/97 & 13519 & 60 & 61.7 & S95T001585 \\
\hline BY -110 & 103 & Sludge & 7 & $8 / 25 / 95$ & 7470 & 40 & 45.3 & S95T001707 \\
\hline BY -110 & 103 & Sludge & 7 & $8 / 25 / 95$ & 7471 & 40 & 60.7 & S95T001708 \\
\hline BY -110 & 103 & Sludge & 8 & $8 / 25 / 95$ & 7645 & 40 & 68.1 & S95T001710 \\
\hline BY -110 & 103 & Sludge & 8 & $8 / 25 / 95$ & 7646 & 40 & 60.5 & S95T001711 \\
\hline BY -110 & 103 & Sludge & 8 & 9/9/97 & 13535 & 60 & 40.8 & S95T001603 \\
\hline BY -110 & 103 & Sludge & 8 & $9 / 26 / 97$ & 13461 & 60 & 19.8 & na \\
\hline BY -110 & 103 & Sludge & 9 & $8 / 25 / 95$ & 7649 & 40 & 29.1 & S95T001714 \\
\hline BY -110 & 103 & Sludge & 9 & $8 / 25 / 95$ & 7650 & 40 & 47.5 & S95T001715 \\
\hline \multirow[t]{2}{*}{ BY -110} & 103 & Sludge & Comp & $2 / 2 / 96$ & 9208 & 60 & 11.5 & S96T000406 \\
\hline & & & & & & Total Weight & 637.3 & \\
\hline BY -110 & 106 & Salt & 1 & $8 / 29 / 95$ & 7655 & 40 & 39.5 & S95T001837 \\
\hline \multirow[t]{2}{*}{ BY-110 } & 106 & Salt & 2 & $9 / 6 / 95$ & $7426 \mathrm{~A}$ & 40 & 10.6 & S95T001946 \\
\hline & & & & & & Total Weight & 50.1 & \\
\hline \multirow[t]{2}{*}{ BY -110} & 107 & DL & $2 \mathrm{~A}$ & $9 / 18 / 97$ & 13477 & 60 & 84.4 & \\
\hline & & & & & & Total Weight & 84.4 & \\
\hline BY -110 & 107 & Salt & 2 & $9 / 7 / 95$ & 7661 & 40 & 23.3 & S95T001960 \\
\hline BY -110 & 107 & Salt & $2 \mathrm{~A}$ & 9/7/95 & 7662 & 40. & 21.2 & S9ST001981 \\
\hline BY-110 & 107 & Salt & 3 & $8 / 31 / 95$ & 7624 & 250 & 45 & S95T002023 \\
\hline BY-110 & 107 & Salt & 3 & $9 / 13 / 95$ & 7676 & 40 & 32.8 & S95T002078 \\
\hline BY-110 & 107 & Salt & 4 & $9 / 14 / 95$ & 7677 & 40 & 36.2 & S95T002079 \\
\hline BY -110 & 107 & Salt & 4 & $9 / 15 / 97$ & 13158 & 60 & 33.5 & S95T002023 \\
\hline BY -110 & 107 & Salt & 5 & $9 / 19 / 95$ & 7699 & 40 & 41.6 & S95T002080 \\
\hline BY-110 & 107 & Salt & 5 & $9 / 19 / 95$ & 7700 & 40 & 63.7 & S95T002128 \\
\hline BY-110 & 107 & Salt & 6 & 9/19/95 & 7697 & 40 & 75.7 & S95T002081 \\
\hline BY-110 & 107 & Salt & 6 & $9 / 19 / 95$ & 7698 & 40 & 67.6 & S95T002082 \\
\hline BY-110 & 107 & Salt & 6 & 9/9/97 & 13528 & 60 & 98.6 & S95T002033 \\
\hline BY -110 & 107 & Salt & 8 & 9/20/95 & 7704 & 40 & 73.3 & S95T002132 \\
\hline BY-110 & 107 & Salt & 8 & $9 / 20 / 95$ & 7705 & 40 & 9.7 & S95T002133 \\
\hline \multirow[t]{2}{*}{ BY -110} & 107 & Salt & Comp & $2 / 2 / 96$ & 9209 & 60 & 16.2 & S96T000407 \\
\hline & & & & & & Total Weight & 638.4 & \\
\hline
\end{tabular}


Vials in 11A1 and 11A2

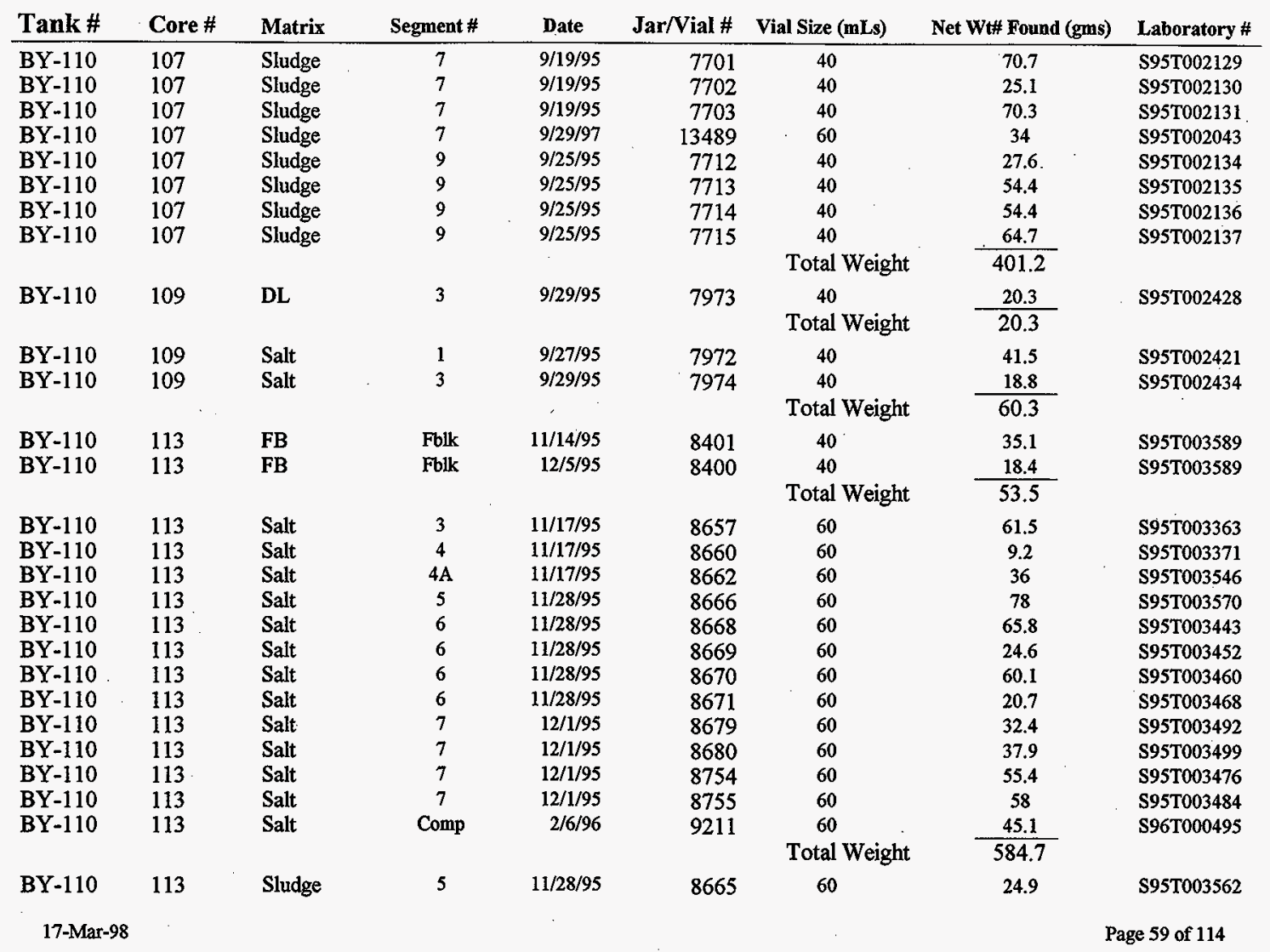


Vials in 11A1 and 11A2

\begin{tabular}{|c|c|c|c|c|c|c|c|c|}
\hline Tank \# & Core\# & Matrix & Segment \# & Date & Jar/Vial \# & Vial Size (mLs) & Net Wt\# Found (gms) & Laboratory \# \\
\hline BY-110 & 113 & Sludge & $5 \mathrm{~A}$ & $11 / 27 / 95$ & 8667 & 60 & 101.4 & S95T003436 \\
\hline BY -110 & 113 & Sludge & 8 & $10 / 26 / 95$ & 8485 & 60 & 46.2 & S95T003180 \\
\hline $\mathrm{BY}-110$ & 113 & Sludge & 8 & $10 / 26 / 95$ & 8486 & 60 & 70 & S95T003189 \\
\hline $\mathrm{BY}-110$ & 113 & Sludge & 8 & $10 / 26 / 95$ & 8487 & 60 & 58.4 & S $95 T 003192$ \\
\hline BY-110 & 113 & Sludge & 8 & $10 / 26 / 95$ & 8488 & 60 & 26.4 & S95T003195 \\
\hline BY-110 & 113 & Sludge & Comp & $2 / 5 / 96$ & 9210 & $\begin{array}{l}60 \\
\text { Total Weight }\end{array}$ & $\frac{9.5}{336.8}$ & S96T000408 \\
\hline BY-110 & 92 & DL & 6 & $7 / 17 / 95$ & 7090 & 125 & 54.14 & \$95T001349 \\
\hline BY -110 & 92 & $\mathrm{DL}$ & 6 & $9 / 7 / 95$ & 7664 & 40 & 33.3 & S95T001810 \\
\hline BY-110 & 92 & $\mathrm{DL}$ & 7 & $7 / 17 / 95$ & 7353 & 250 & 243.5 & S95T001352 \\
\hline BY-110 & 92 & DL & 7 & 9/8/95 & 7667 & $\begin{array}{l}40 \\
\text { Total Weight }\end{array}$ & $\frac{60.7}{391.64}$ & S9ST001811 \\
\hline BY -110 & 92 & Salt & 5 & $9 / 7 / 95$ & 7663 & 40 & 19 & S95T001805 \\
\hline BY-110 & 92 & Salt & 6 & 9/8/95 & 7666 & 40 & 81.7 & S95T001807 \\
\hline BY -110 & 92 & Salt & 6 & 9/8/95 & 7720 & 125 & 32.7 & S95T001351 \\
\hline \multirow[t]{2}{*}{ BY-110 } & 92 & Salt & Comp & $1 / 22 / 96$ & 9204 & 60 & 7.2 & S96T000399 \\
\hline & & & & & & Total Weight & 140.6 & \\
\hline BY-110 & 92 & Sludge & 7 & $7 / 17 / 95$ & 7092 & $\begin{array}{c}125 \\
\text { Total Weight }\end{array}$ & $\frac{34.6}{34.6}$ & S95T001353 \\
\hline BY-110 & 95 & Salt & 4 & 9/8/95 & 7669 & 40 & 18 & S95T001778 \\
\hline BY -110 & 95 & Salt & 5 & $9 / 11 / 95$ & 7673 & 40 & 38.1 & S95T001779 \\
\hline BY -110 & 95 & Salt & 5 & $9 / 11 / 95$ & 7675 & 40 & 39.4 & \$95T001780 \\
\hline BY -110 & 95 & Salt & 6 & $9 / 8 / 95$ & 7670 & 40 & 29.4 & S95T001781 \\
\hline \multirow[t]{2}{*}{ BY-110 } & 95 & Salt & Comp & $1 / 20 / 96$ & 9205 & 60 & 13.1 & S96T000400 \\
\hline & & & & & & Total Weight & 138 & \\
\hline BY-110 & 96 & Salt & 1 & $10 / 18 / 95$ & 8375 & 60 & 50 & S95T002876 \\
\hline BY -110 & 96 & Salt & 2 & $10 / 18 / 95$ & 8376 & 60 & 90.8 & S95T002884 \\
\hline BY -110 & 96 & Salt & 2 & 9/9/97 & 13533 & 60 & 52.2 & S95T002886 \\
\hline BY-110 & 96 & Salt & 3 & $10 / 19 / 95$ & 8377 & 60 & 68.9 & S95T002891 \\
\hline \multirow[t]{2}{*}{ BY -110} & 96 & Salt & 3 & $10 / 19 / 95$ & 8378 & $\begin{array}{l}60 \\
0\end{array}$ & $\begin{array}{r}43.7 \\
20.6\end{array}$ & S95T002898 \\
\hline & & & & & & Total Weight & 305.6 & \\
\hline
\end{tabular}


Vials in 11A1 and 11A2

\begin{tabular}{|c|c|c|c|c|c|c|c|c|}
\hline Tank \# & Core \# & Matrix & Segment \# & Date & Jar/Vial \# & Vial Sixe (mLs) & Net Wt\# Found (gms) & Laboratory \# \\
\hline BY-111 & 168 & $\mathrm{DL}$ & 6 & $10 / 11 / 96$ & 11491 & $\begin{array}{c}60 \\
\text { Total Weight }\end{array}$ & $\begin{array}{l}59.4 \\
59.4\end{array}$ & S96T005315 \\
\hline $\begin{array}{l}\text { BY-111 } \\
\text { BY-111 }\end{array}$ & $\begin{array}{l}168 \\
168\end{array}$ & $\begin{array}{l}\text { FB } \\
\text { FB }\end{array}$ & $\begin{array}{l}\text { Folk } \\
\text { Folk }\end{array}$ & $\begin{array}{l}9 / 25 / 96 \\
10 / 2 / 96\end{array}$ & $\begin{array}{l}11193 \\
11391\end{array}$ & $\begin{array}{l}250 \\
60 \\
\text { Total Weight }\end{array}$ & $\begin{array}{c}166.4 \\
55.3 \\
221.7\end{array}$ & $\begin{array}{l}\text { S96T005297 } \\
\text { S96T005299 }\end{array}$ \\
\hline $\begin{array}{l}\text { BY-111 } \\
\text { BY-111 }\end{array}$ & $\begin{array}{l}168 \\
168\end{array}$ & $\begin{array}{l}\text { LL } \\
\text { LL }\end{array}$ & $\begin{array}{c}6 \\
6 \mathrm{~A}\end{array}$ & $\begin{array}{r}9 / 30 / 96 \\
10 / 12 / 96\end{array}$ & $\begin{array}{l}11116 \\
11117\end{array}$ & $\begin{array}{c}40 \\
40 \\
\text { Total Weight }\end{array}$ & $\begin{array}{r}35.7 \\
37.5 \\
73.2\end{array}$ & $\begin{array}{l}\text { \$96T005317 } \\
\text { S96T005154 }\end{array}$ \\
\hline $\begin{array}{l}\text { BY-111 } \\
\text { BY-111 } \\
\text { BY-111 } \\
\text { BY-111 } \\
\text { BY-111 } \\
\text { BY-111 } \\
\text { BY-111 }\end{array}$ & $\begin{array}{l}168 \\
168 \\
168 \\
168 \\
168 \\
168 \\
168\end{array}$ & $\begin{array}{l}\text { Salt } \\
\text { Salt } \\
\text { Salt } \\
\text { Salt } \\
\text { Salt } \\
\text { Salt } \\
\text { Salt }\end{array}$ & $\begin{array}{c}1 \\
1 \\
2 \\
3 \\
3 \\
4 \\
5 \mathrm{~A}\end{array}$ & $\begin{array}{r}9 / 18 / 96 \\
10 / 10 / 96 \\
10 / 11 / 96 \\
9 / 18 / 96 \\
10 / 9 / 96 \\
10 / 2 / 96 \\
9 / 30 / 96\end{array}$ & $\begin{array}{l}11371 \\
11493 \\
11492 \\
10983 \\
11490 \\
11489 \\
11375\end{array}$ & $\begin{array}{c}60 \\
60 \\
60 \\
125 \\
60 \\
60 \\
60 \\
\text { Total Weight }\end{array}$ & $\begin{array}{c}17.8 \\
37.2 \\
51.9 \\
11.6 \\
59.5 \\
85.2 \\
61.1 \\
324.3\end{array}$ & $\begin{array}{l}\text { S96T005164 } \\
\text { S96T005392 } \\
\text { S96T005149 } \\
\text { S96T005155 } \\
\text { S96T005159 } \\
\text { S96T005302 } \\
\text { S96T005309 }\end{array}$ \\
\hline $\begin{array}{l}\text { BY-111 } \\
\text { BY-111 }\end{array}$ & $\begin{array}{l}171 \\
171\end{array}$ & $\begin{array}{l}\mathrm{DL} \\
\mathrm{DL}\end{array}$ & $\begin{array}{l}5 \\
6\end{array}$ & $\begin{array}{r}10 / 2 / 96 \\
10 / 16 / 96\end{array}$ & $\begin{array}{l}11475 \\
11481\end{array}$ & $\begin{array}{l}60 \\
60 \\
\text { Total Weight }\end{array}$ & $\begin{array}{c}72.5 \\
102.9 \\
175.4\end{array}$ & $\begin{array}{l}\text { S96T005345 } \\
\text { S96T005358 }\end{array}$ \\
\hline $\begin{array}{l}\text { BY-111 } \\
\text { BY-111 } \\
\text { BY-111 } \\
\text { BY-111 } \\
\text { BY-111 } \\
\text { BY-111 } \\
\text { BY-111 } \\
\text { BY-111 }\end{array}$ & $\begin{array}{l}171 \\
171 \\
171 \\
171 \\
171 \\
171 \\
171 \\
171\end{array}$ & $\begin{array}{l}\text { Salt } \\
\text { Salt } \\
\text { Salt } \\
\text { Salt } \\
\text { Salt } \\
\text { Salt } \\
\text { Salt } \\
\text { Salt }\end{array}$ & $\begin{array}{l}1 \\
4 \\
5 \\
6 \\
6 \\
7 \\
7 \\
7\end{array}$ & $\begin{array}{r}10 / 13 / 96 \\
10 / 2 / 96 \\
10 / 2 / 96 \\
10 / 2 / 96 \\
10 / 16 / 96 \\
10 / 10 / 96 \\
10 / 17 / 96 \\
10 / 17 / 96\end{array}$ & $\begin{array}{l}11498 \\
11474 \\
11476 \\
11477 \\
11479 \\
11530 \\
11482 \\
11483\end{array}$ & $\begin{array}{c}60 \\
60 \\
60 \\
60 \\
60 \\
250 \\
60 \\
60 \\
\text { Total Weight }\end{array}$ & $\begin{array}{c}46.9 \\
59 \\
83.9 \\
65 \\
92.5 \\
87.1 \\
108.5 \\
109.8 \\
652.7\end{array}$ & $\begin{array}{l}\text { S96T005322 } \\
\text { S96T005334 } \\
\text { S96T005341 } \\
\text { S96T005348 } \\
\text { S96T005351 } \\
\text { S96T005382 } \\
\text { S96T005386 } \\
\text { S96T005385 }\end{array}$ \\
\hline
\end{tabular}

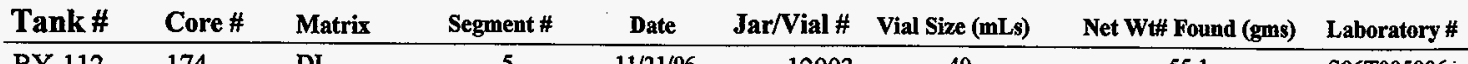

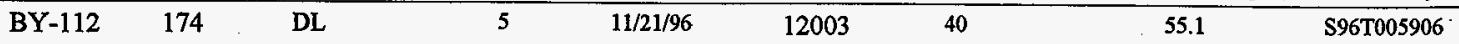


Vials in 11A1 and 11A2

\begin{tabular}{|c|c|c|c|c|c|c|c|c|}
\hline Tank\# & Core \# & Matrix & Segment \# & Date & Jar/Vial \# & Vial Size (mLs) & Net Wt\# Found (gms) & Laboratory \# \\
\hline & & & & & & Total Weight & 55.1 & . \\
\hline BY-112 & 174 & Salt & 1 & $11 / 20 / 96$ & 11799 & 60 & 32.1 & S96T005881 \\
\hline BY -112 & 174 & Salt & 2 & $11 / 4 / 96$ & 11704 & 125 & 30.2 & S96T005866 \\
\hline BY -112 & 174 & Salt & 2 & $11 / 20 / 96$ & 11800 & 60 & 57.6 & S96T005882 \\
\hline BY-112 & 174 & Salt & 3 & $11 / 20 / 96$ & 11801 & 60 & 58.2 & S96T005883 \\
\hline BY -112 & 174 & Salt & 4 & $11 / 20 / 96$ & 11802 & 60 & 98.6 & S96T005884 \\
\hline BY -112 & 174 & Salt & 5 & $11 / 21 / 96$ & 11807 & 60 & 76.7 & S96T005886 \\
\hline BY -112 & 174 & Salt & 5 & $11 / 21 / 96$ & 11808 & 60 & 78.3 & S96T005885 \\
\hline BY-112 & 174 & Salt & 6 & $11 / 4 / 96$ & 11565 & 250 & 102.8 & S96T005872 \\
\hline BY -112 & 174 & Salt & 6 & $11 / 21 / 96$ & 11809 & 60 & 84 & S96T005888 \\
\hline \multirow[t]{2}{*}{ BY-112 } & 174 & Salt & 6 & $11 / 21 / 96$ & 11810 & 60 & 84.7 & S96T005887 \\
\hline & & & & & & Total Weight & 703.2 & \\
\hline BY -112 & 177 & DL & 5 & $11 / 21 / 96$ & 11805 & 60 & 114.9 & S96T005945 \\
\hline \multirow[t]{2}{*}{ BY-112 } & 177 & DL & 5 & $11 / 21 / 96$ & 11991 & 40 & 20.2 & S96T005946 \\
\hline & & & & & & Total Weight & 135.1 & \\
\hline BY-112 & 177 & FB & Fblk & $10 / 25 / 96$ & 11552 & 250 & 161.2 & S96T005908 \\
\hline \multirow[t]{2}{*}{ BY-112 } & 177 & FB & Folk & $11 / 19 / 96$ & 11792 & 60 & 63.5 & S96T005910 \\
\hline & & & & & & Total Weight & 224.7 & \\
\hline BY-112 & 177 & Salt & 1 & $11 / 19 / 96$ & 11793 & .60 & 21.2 & S96T005923 \\
\hline BY-112 & 177 & Salt & 2 & $11 / 20 / 96$ & 11787 & 60 & 41.9 & S96T005924 \\
\hline BY-112 & 177 & Salt & 3 & $11 / 20 / 96$ & 11794 & 60 & 55.4 & S96T005925 \\
\hline BY -112 & 177 & Salt & 4 & $11 / 6 / 96$ & 11763 & 60 & 27 & S96T005914 \\
\hline BY -112 & 177 & Salt & 4 & $11 / 21 / 96$ & 11803 & 60 & 125.3 & S96T005926 \\
\hline BY-112 & 177 & Salt & 5 & $11 / 21 / 96$ & 11806 & 60 & 124.9 & S96T005947 \\
\hline \multirow[t]{2}{*}{ BY-112 } & 177 & Salt & 6 & $11 / 21 / 96$ & 11804 & 60 & 89.8 & S96T005927 \\
\hline & & & & & & Total Weight & 485.5 & \\
\hline
\end{tabular}

\begin{tabular}{|c|c|c|c|c|c|c|c|c|}
\hline Tank\# & Core \# & Matrix & Segment \# & Date & Jar/Vial \# & Vial Size (mLs) & Net Wt\# Found (gms) & Laboratory \# \\
\hline C-101 & & Sludge & Aug-19 & $3 / 1 / 96$ & 9347 & 60 & 74.1 & S95T000826 \\
\hline C-101 & & Sludge & Aug-19 & $3 / 1 / 96$ & 9348 & 60 & 13.7 & S95T000830 \\
\hline & & & & & & Total Weight & 87.8 & \\
\hline
\end{tabular}


Vials in 11A1 and 11A2

\begin{tabular}{lllcccccc} 
Tank \# & Core \# & Matrix & Segment \# & Date & Jar/Vial \# & Vial Size (mLs) & Net Wt\# Found (gms) & Laboratory \# \\
\hline C-101 & R8 & DL & Aug-19 & $4 / 14 / 95$ & 6893 & 40 & 15.9 & S95T000821 \\
& & & & & & Total Weight & 15.9 & \\
C-101 & R8 & Sludge & Aug-19 & $4 / 14 / 95$ & 6908 & 40 & 57.8 & S95T000833 \\
C-101 & R8 & Sludge & Aug-19 & $4 / 14 / 95$ & 6909 & 40 & 60 & S95T000829 \\
C-101 & R8 & Sludge & Aug-19 & $4 / 14 / 95$ & 7151 & 40 & T.3 & S95T000825
\end{tabular}

\begin{tabular}{lllcccccc} 
Tank \# & Core \# & Matrix & Segment \# & Date & Jar/Vial \# & Vial Size (mLs) & Net Wt\# Found (gms) & Laboratory \# \\
\hline C-102 & R3 & Sludge & Auger & $1 / 16 / 95$ & 6364 & $\begin{array}{c}125 \\
\text { Total Weight }\end{array}$ & $\frac{26.2}{26.2}$.
\end{tabular}

\begin{tabular}{|c|c|c|c|c|c|c|c|c|}
\hline Tank\# & Core \# & Matrix & Segment \# & Date & Jar/Vial \# & Vial Size (mLs) & Net Wt\# Found (gms) & Laboratory \# \\
\hline C- 103 & 63 & DL & 1 & $11 / 4 / 94$ & 6236 & 250 & 140.9 & S94T000204 \\
\hline C-103 & 63 & DL & 1 & $11 / 10 / 94$ & 7024 & 40 & 13.2 & $\mathrm{~S} 94 \mathrm{~T} 000203$ \\
\hline C-103 & 63 & DL & 2 & $1 / 24 / 95$ & 6371 & 250 & 70.8 & \\
\hline C-103 & 63 & DL & 2 & $1 / 25 / 95$ & 6452 & 40 & 22.1 & S95T000070 \\
\hline C- 103 & 63 & DL & 4 & $2 / 3 / 95$ & 6396 & 40 & 26.95 & S95T000076 \\
\hline \multirow[t]{2}{*}{ C-103 } & 63 & DL & 4 & 9/3/97 & 13512 & 60 & 5.5 & \\
\hline & & & & & & Total Weight & 279.45 & \\
\hline C-103 & 63 & Sludge & 3 & $1 / 26 / 95$ & 6454 & 40 & 40.9 & S95T000073 \\
\hline C-103 & 63 & Sludge & 3 & $1 / 27 / 95$ & 6394 & 40 & 18.6 & S95T000074 \\
\hline C-103 & 63 & Sludge & 3 & $9 / 3 / 97$ & 13514 & 60 & 12.1 & S95T000065 \\
\hline C-103 & 63 & Sludge & 3 & 9/8/97 & 13261 & 40 & 12.6 & S95T000064 \\
\hline C-103 & 63 & Sludge & 4 & $1 / 27 / 95$ & 6395 & 40 & 56.7 & \$95T000075 \\
\hline C-103 & 63 & Sludge & 4 & 9/8/97 & 13260 & 40 & 17.1 & S95T000069 \\
\hline \multirow{2}{*}{ C-103 } & 63 & Sludge & Comp & $3 / 15 / 95$ & 6572 & 125 & 42.8 & \\
\hline & & & & & & Total Weight & 200.8 & \\
\hline C-103 & 66 & DL & 2 & $2 / 27 / 95$ & 6541 & 250 & 156.3 & \\
\hline C-103 & 66 & $\mathrm{DL}$ & 2 & $2 / 27 / 95$ & 6557 & 125 & 63.4 & \\
\hline C- 103 & 66 & $\mathrm{DL}$ & 2 & $3 / 3 / 95$ & 6414 & 40 & 32.6 & S95T000404 \\
\hline C-103 & 66 & DL & 3 & $2 / 27 / 95$ & 6543 & 250 & 163.1 & \\
\hline
\end{tabular}


Vials in 11A1 and 11A2

\begin{tabular}{|c|c|c|c|c|c|c|c|c|}
\hline Tank\# & Core \# & Matrix & Segment \# & Date & Jar/Vial \# & Vial Size (mLs) & Net Wt\# Found (gms) & Laboratory \# \\
\hline C-103 & 66 & DL & 3 & $2 / 27 / 95$ & 6563 & 125 & 8.7 & \\
\hline C-103 & 66 & DL & 3 & $3 / 3 / 95$ & 6408 & 40 & 5.6 & S95T000405 \\
\hline C-103 & 66 & DL & 3 & $8 / 19 / 97$ & 13085 & 60 & 26.6 & S97M000054 \\
\hline C- 103 & 66 & DL & 4 & $2 / 27 / 95$ & 6546 & 250 & 10.2 & \\
\hline \multirow[t]{2}{*}{ C- 103} & 66 & $\mathrm{DL}$ & 4 & $3 / 3 / 95$ & 6412 & 40 & 10.8 & S95T000406 \\
\hline & & & & & & Total Weight & $47 \overline{7.3}$ & \\
\hline C-103 & 66 & FB & Fblk & $2 / 24 / 95$ & 6540 & 250 & 123.7 & \\
\hline \multirow[t]{2}{*}{ C- 103} & 66 & FB & Fblk & $2 / 24 / 95$ & 6562 & 125 & 79.7 & \\
\hline & & & & & & Total Weight & 203.4 & \\
\hline C-103 & 66 & Sludge & 3 & $3 / 28 / 95$ & 6746 & 40 & 16.3 & S95T000408 \\
\hline C-103 & 66 & Sludge & 4 & $2 / 27 / 95$ & 6558 & 125 & 3.9 & \\
\hline \multirow[t]{2}{*}{ C-103 } & 66 & Sludge & 4 & $3 / 6 / 95$ & 6440 & 40 & 33.5 & S95T000409 \\
\hline & & & & & & Total Weight & 53.7 & \\
\hline Tank \# & Core \# & Matrix & Segment \# & Date & Jar/Vial \# & Vial Size (mLs) & Net Wt\# Found (gms) & Laboratory \# \\
\hline C-104 & 162 & $\mathrm{DL}$ & 3 & $8 / 17 / 96$ & 11099 & 40 & 14.6 & S96T004364 \\
\hline \multirow[t]{2}{*}{ C-104 } & 162 & $\mathrm{DL}$ & Fblk & $9 / 15 / 96$ & 11111 & 40 & 33 & S96T004829 \\
\hline & & & & & & Total Weight & 47.6 & \\
\hline \multirow[t]{2}{*}{ C-104 } & 162 & FB & Folk & $9 / 3 / 96$ & 11082 & 250 & 140.1 & S96T004825 \\
\hline & & & & & & Total Weight & 140.1 & \\
\hline C-104 & 162 & Sludge & 1 & $9 / 3 / 96$ & 11087 & 250 & 40.5 & S96T004835 \\
\hline C-104 & 162 & Sludge & 1 & $9 / 3 / 96$ & 11088 & 250 & 68.1 & S96T004832 \\
\hline C-104 & 162 & Sludge & 1 & $9 / 15 / 96$ & 11350 & 60 & 45.5 & S96T004844 \\
\hline C-104 & 162 & Sludge & 1 & 9/15/96 & 11351 & 60 & 43.2 & S96T்004845 \\
\hline C-104 & 162 & Sludge & 2 & $9 / 3 / 96$ & 11089 & 250 & 72.3 & S96T004836 \\
\hline C-104 & 162 & Sludge & 2 & $9 / 15 / 96$ & 11352 & 60 & 79.6 & S96T004846 \\
\hline C-104 & 162 & Sludge & 2 & $9 / 15 / 96$ & 11354 & 60 & 72.8 & S96T004847 \\
\hline C-104 & 162. & Sludge & 2 & $9 / 25 / 97$ & 13459 & 60 & 28.9 & S96T004833 \\
\hline C-104 & 162 & Sludge & 3 & $8 / 8 / 96$ & 10618 & 250 & 65 & S96T004365 \\
\hline C-104 & 162 & Sludge & 3 & $8 / 17 / 96$ & 11118 & 60 & 61.3 & S96T004359 \\
\hline C-104 & 162 & Sludge & 3 & $8 / 17 / 96$ & 11119 & 60 & 78.4 & S96T004367 \\
\hline C- 104 & 162 & Sludge & 4 & $9 / 3 / 96$ & 11179 & 250 & 75.9 & S96T004834 \\
\hline
\end{tabular}


Vials in 11A1 and 11A2

\begin{tabular}{|c|c|c|c|c|c|c|c|c|}
\hline Tank \# & Core \# & Matrix & Segment \# & Date & Jar/Vial \# & Vial Size (mLs) & Net Wt\# Found (gms) & Laboratory \# \\
\hline C-104 & 162 & Sludge & 4 & $9 / 15 / 96$ & 11355 & 60 & 90.5 & S96T004848 \\
\hline C- 104 & 162 & Sludge & 4 & $9 / 15 / 96$ & 11356 & 60 & 54.1 & S96T004849 \\
\hline C-104 & 162 & Sludge & Comp & $10 / 4 / 96$ & 11392 & 250 & 40.8 & S96T004946 \\
\hline \multirow[t]{2}{*}{ C-104 } & 162 & Sludge & Comp & $10 / 4 / 96$ & 11480 & 60 & 102 & S96T004948 \\
\hline & & & & & & Total Weight & 1018.9 & \\
\hline C-104 & 165 & Sludge & 2 & $8 / 16 / 96$ & 10968 & 60 & 42.5 & S96T004372 \\
\hline C-104 & 165 & Sludge & 2 & $8 / 17 / 96$ & 10969 & 60 & 92.8 & S96T004377 \\
\hline C-104 & 165 & Sludge & 2 & $8 / 7 / 97$ & 10605 & 250 & 78.2 & S96T004370 \\
\hline C- 104 & 165 & Sludge & 3 & $9 / 4 / 96$ & 11085 & 250 & 45.8 & S96T004867 \\
\hline C-104 & 165 & Sludge & 3 & $9 / 16 / 96$ & 11357 & 60 & 51.3 & S96T004882 \\
\hline C-104 & 165 & Sludge & 3 & $9 / 16 / 96$ & 11358 & 60 & 60.3 & S96T004883 \\
\hline C- 104 & 165 & Sludge & 3 & $9 / 16 / 96$ & 11359 & 60 & 97.8 & S96T004884 \\
\hline C-104 & 165 & Sludge & 6 & $9 / 4 / 96$ & 11140 & 125 & 17.5 & S96T004871 \\
\hline C-104 & 165 & Sludge & 6 & $9 / 4 / 96$ & 11244 & 60 & 22.5 & S96T004870 \\
\hline C-104 & 165 & Sludge & 6 & $9 / 16 / 96$ & 11245 & 60 & 6.9 & S96T004865 \\
\hline C-104 & 165 & Sludge & 6 & $9 / 16 / 96$ & 11366 & 60 & 65.9 & S96T004887 \\
\hline C-104 & 165 & Sludge & 6 & $9 / 17 / 96$ & 11367 & 60 & 75.9 & S96T004888 \\
\hline \multirow[t]{2}{*}{ C-104 } & 165 & Sludge & Comp & $10 / 23 / 96$ & 11513 & 60 & 8.8 & S96T004955 \\
\hline & & & & & & Total Weight & 666.2 & \\
\hline
\end{tabular}

\begin{tabular}{|c|c|c|c|c|c|c|c|c|}
\hline Tank \# & Core\# & Matrix & Segment \# & Date & Jar/Vial \# & Vial Size (mLs) & Net Wt\# Found (gms) & Laboratory \# \\
\hline C-105 & & Sludge & Comp & $9 / 26 / 97$ & 13446 & $\begin{array}{c}60 \\
\text { Total Weight }\end{array}$ & $\frac{16}{16}$ & S95T001213 \\
\hline $\begin{array}{l}C-105 \\
C-105\end{array}$ & $\begin{array}{l}72 \\
72\end{array}$ & $\begin{array}{l}\mathrm{DL} \\
\mathrm{DL}\end{array}$ & $\begin{array}{l}1 \\
2\end{array}$ & $\begin{array}{l}3 / 23 / 95 \\
4 / 10 / 95\end{array}$ & $\begin{array}{l}6731 \\
6890\end{array}$ & $\begin{array}{c}40 \\
40 \\
\text { Total Weight }\end{array}$ & $\begin{array}{c}9.1 \\
35.9 \\
45\end{array}$ & $\begin{array}{l}\text { S95T000671 } \\
\text { S95T000679 }\end{array}$ \\
\hline $\begin{array}{l}C-105 \\
C-105 \\
C-105 \\
C-105\end{array}$ & $\begin{array}{l}72 \\
72 \\
72 \\
72\end{array}$ & $\begin{array}{l}\text { Sludge } \\
\text { Sludge } \\
\text { Sludge } \\
\text { Sludge }\end{array}$ & $\begin{array}{l}1 \\
2 \\
3 \\
3\end{array}$ & $\begin{array}{r}4 / 10 / 95 \\
10 / 13 / 95 \\
4 / 10 / 95 \\
4 / 10 / 95\end{array}$ & $\begin{array}{l}6888 \\
8274 \\
6895 \\
6896\end{array}$ & $\begin{array}{c}\begin{array}{c}40 \\
20 \\
40 \\
40\end{array} \\
\text { Total Weight }\end{array}$ & \begin{tabular}{|c|}
30.4 \\
2.8 \\
45.2 \\
58.6 \\
137
\end{tabular} & $\begin{array}{l}\text { S95T000675 } \\
\text { S95T002854 } \\
\text { S95T000697 } \\
\text { S95T000703 }\end{array}$ \\
\hline $\begin{array}{l}\text { C-105 } \\
\text { 17-Mar-98 }\end{array}$ & 76 & DL & 2 & $3 / 29 / 95$ & 6700 & 250 & 17 & $\begin{array}{l}\text { S95T000640 } \\
\text { ge } 65 \text { of } 114\end{array}$ \\
\hline
\end{tabular}


Vials in 11A1 and 11A2

\begin{tabular}{lllcccccc} 
Tank \# & Core \# & Matrix & Segment \# & Date & Jar/Vial \# & Vial Size (mLs) & Net Wt\# Found (gms) & Laboratory \# \\
\hline C-105 & 76 & DL & 2 & $4 / 7 / 95$ & 6885 & 40 & 48.8 & S95T000652 \\
C-105 & 76 & DL & 3 & $3 / 30 / 95$ & 6747 & 40 & S1.3 & S95T000521
\end{tabular}

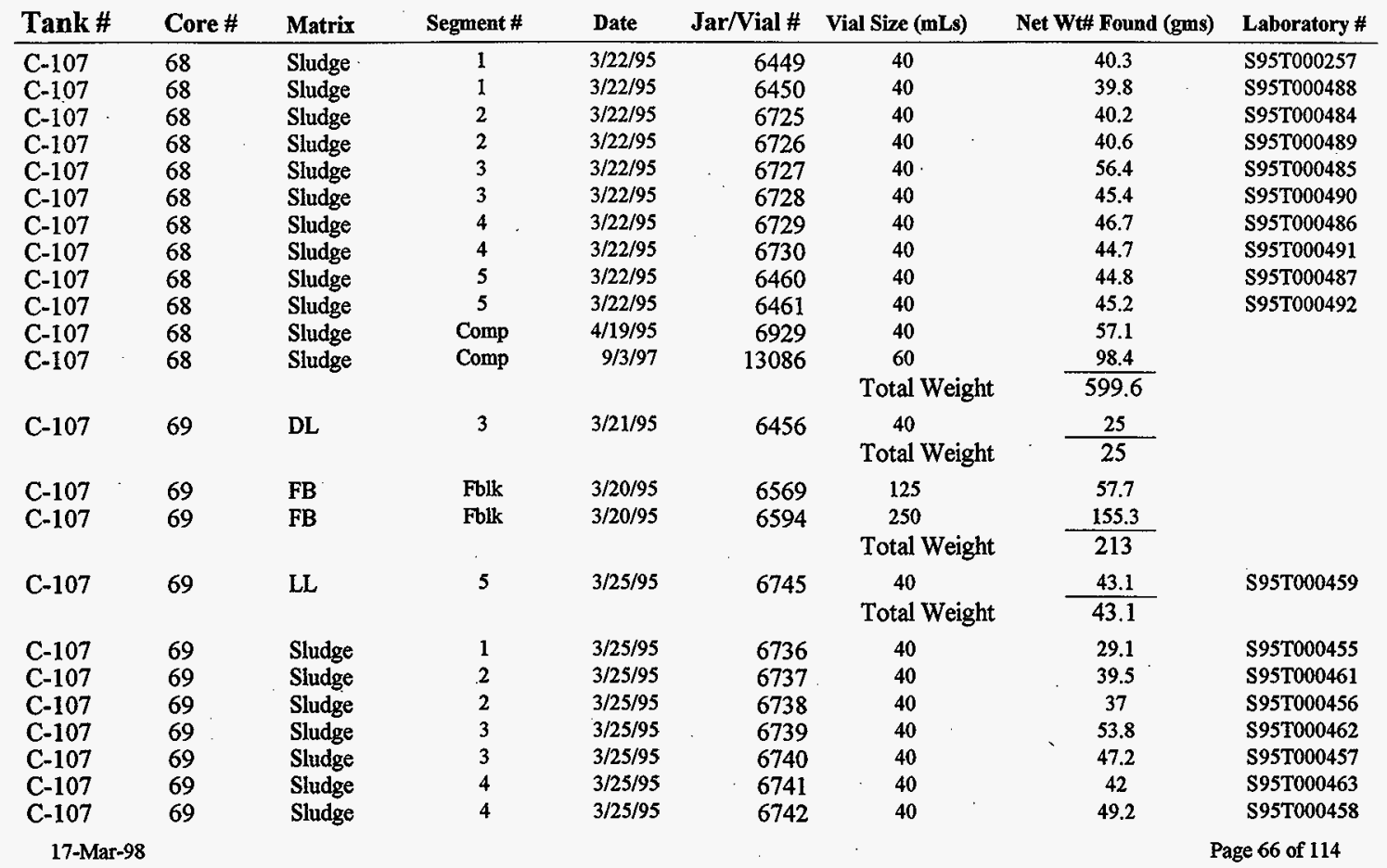


Vials in 11A1 and 11A2

\begin{tabular}{lllcccccc} 
Tank \# & Core \# & Matrix & Segment \# & Date & Jar/Vial \# & Vial Size (mLs) & Net Wt\# Found (gms) & Laboratory \# \\
\hline C-107 & 69 & Sludge & 5 & $3 / 25 / 95$ & 6743 & 40 & 46.5 & S95T000464 \\
C-107 & 69 & Sludge & Comp & $4 / 14 / 95$ & 6773 & 250 & 108.3 & 5.8 \\
C-107 & 69 & Sludge & Comp & $4 / 19 / 95$ & 6928 & 40 & 56.8 &
\end{tabular}

\begin{tabular}{|c|c|c|c|c|c|c|c|c|}
\hline Tank \# & Core \# & Matrix & Segment \# & Date & Jar/Vial \# & Vial Size (mLs) & Net Wt\# Found (gms) & Laboratory \# \\
\hline C-108 & & Sludge & Aug- 12 & $9 / 22 / 97$ & 13266 & $\begin{array}{l}40 \\
\text { Total Weight }\end{array}$ & $\begin{array}{r}7.3 \\
7.3\end{array}$ & \\
\hline $\begin{array}{l}\text { C-108 } \\
\text { C-108 } \\
\text { C-108 } \\
\text { C-108 } \\
\text { C-108 } \\
\text { C-108 } \\
C-108\end{array}$ & $\begin{array}{l}\text { R4 } \\
\text { R4 } \\
\text { R4 } \\
\text { R4 } \\
\text { R4 } \\
\text { R4 } \\
\text { R4 }\end{array}$ & $\begin{array}{l}\text { Sludge } \\
\text { Sludge } \\
\text { Sludge } \\
\text { Sludge } \\
\text { Sludge } \\
\text { Sludge } \\
\text { Sludge }\end{array}$ & $\begin{array}{l}\text { Auger } \\
\text { Auger } \\
\text { Auger } \\
\text { Auger } \\
\text { Auger } \\
\text { Auger } \\
\text { Comp }\end{array}$ & $\begin{array}{r}12 / 15 / 94 \\
12 / 15 / 94 \\
12 / 15 / 94 \\
12 / 15 / 94 \\
12 / 15 / 94 \\
12 / 15 / 94 \\
9 / 22 / 97\end{array}$ & $\begin{array}{r}6162 \\
6360 \\
6377 \\
6378 \\
6379 \\
6380 \\
13441\end{array}$ & $\begin{array}{c}125 \\
125 \\
40 \\
40 \\
40 \\
40 \\
60 \\
\text { Total Weight }\end{array}$ & $\begin{array}{c}15.6 \\
29.3 \\
13.9 \\
92.1 \\
14.6 \\
19.9 \\
47 \\
232.4\end{array}$ & \\
\hline $\begin{array}{l}\text { C-108 } \\
\text { C-108 } \\
\text { C-108 } \\
\text { C-108 }\end{array}$ & $\begin{array}{l}\text { R7 } \\
\text { R7 } \\
\text { R7 } \\
\text { R7 }\end{array}$ & $\begin{array}{l}\text { Sludge } \\
\text { Sludge } \\
\text { Sludge } \\
\text { Sludge }\end{array}$ & $\begin{array}{l}\text { Auger } \\
\text { Auger } \\
\text { Auger } \\
\text { Auger }\end{array}$ & $\begin{array}{r}12 / 7 / 94 \\
12 / 7 / 94 \\
12 / 7 / 94 \\
12 / 18 / 94\end{array}$ & $\begin{array}{l}7034 \\
7037 \\
7038 \\
7039\end{array}$ & $\begin{array}{c}40 \\
40 \\
40 \\
40 \\
\text { Total Weight }\end{array}$ & $\begin{array}{c}12.6 \\
9.5 \\
13.6 \\
18.1 \\
53.8\end{array}$ & \\
\hline
\end{tabular}

\begin{tabular}{|c|c|c|c|c|c|c|c|c|}
\hline Tank \# & Core \# & Matrix & Segment \# & Date & Jar/Vial \# & Vial Sixe (mLs) & Net Wt\# Found (gms) & Laboratory \# \\
\hline $\mathrm{C}-111$ & & Sludge & 5-AUG-01 & $9 / 18 / 97$ & 13481 & 60 & 45.1 & \\
\hline C-111 & & Sludge & 5-AUG-01 & $9 / 18 / 97$ & 13482 & 60 & 74.8 & \\
\hline C-111 & & Sludge & Aug-16 & $9 / 17 / 97$ & 13485 & 60 & 46.7 & na \\
\hline $\mathrm{C}-111$ & & Sludge & Aug-17 & $9 / 16 / 97$ & 13479 & 60 & 40.7 & na \\
\hline & & & & & & Total Weight & 207.3 & \\
\hline \multirow[t]{2}{*}{ C-111 } & 60 & Salt & 2 & $9 / 4 / 97$ & 13524 & 60 & 9.8 & \\
\hline & & & & & & Total Weight & 9.8 & \\
\hline
\end{tabular}


Vials in 11A1 and 11A2

\begin{tabular}{lllcccccc} 
Tank \# & Core \# & Matrix & Segment \# & Date & Jar/Vial \# & Vial Size (mLs) & Net Wt\# Found (gms) & Laboratory \# \\
\hline C-201 & 221 & Sludge & Comp & $11 / 7 / 97$ & 13620 & $\begin{array}{c}60 \\
\text { Total Weight }\end{array}$ & $\frac{33.8}{33.8}$ & S97T002250
\end{tabular}

\begin{tabular}{|c|c|c|c|c|c|c|c|c|}
\hline Tank\# & Core \# & Matrix & Segment \# & Date & Jar/Vial \# & Vial Size (mLs) & Net Wt\# Found (gms) & Laboratory \# \\
\hline C-202 & 222 & Sludge & Comp & $1 / 15 / 98$ & 12950 & 250 & 61.9 & S98T000035 \\
\hline C-202 & 222 & Sludge & Comp & $1 / 15 / 98$ & 13673 & $\begin{array}{c}60 \\
\text { Total Weight }\end{array}$ & $\frac{13.5}{75.4}$ & S98T000038 \\
\hline
\end{tabular}

\begin{tabular}{|c|c|c|c|c|c|c|c|c|}
\hline Tank \# & Core \# & Matrix & Segment \# & Date & Jar/Vial \# & Vial Size (mLs) & Net Wt\# Found (gms) & Laboratory \# \\
\hline C-203 & & Sludge & $5-\mathrm{AUG}-02$ & 9/9/97 & 13531 & 60 & 108.3 & S95T000798 \\
\hline C-203 & & Sludge & Aug-20 & $7 / 19 / 95$ & 7412 & 40 & 31.3 & \\
\hline \multirow[t]{2}{*}{$\mathrm{C}-203$} & & Sludge & Aug-21 & $9 / 12 / 97$ & 13468 & 60 & 109.6 & S95T000802 \\
\hline & & & & & & Total Weight & 249.2 & \\
\hline \multirow[t]{2}{*}{ C-203 } & "R7" & Sludge & Aug-20 & $7 / 19 / 95$ & 7411 & 40 & 24.7 & S95T000809 \\
\hline & & & & & & Total Weight & 24.7 & \\
\hline C-203 & 203 & Sludge & 5-AUG-02 & 9/9/97 & 11836 & 125 & 130.2 & \\
\hline \multirow[t]{2}{*}{$\mathrm{C}-203$} & 203 & Sludge & 5-AUG-02 & 9/9/97 & 11837 & 125 & 139.1 & \\
\hline & & & & & & Total Weight & 269.3 & \\
\hline $\mathrm{C}-203$ & R7 & Sludge & Aug-21 & $4 / 13 / 95$ & 7160 & 40 & 61.5 & S95T000805 \\
\hline$C-203$ & R7 & Sludge & Aug-21 & $4 / 13 / 95$ & 7161 & 40 & 68.5 & S95T000801 \\
\hline \multirow[t]{2}{*}{ C-203 } & R7 & Sludge & Aug-21 & $5 / 20 / 96$ & 10120 & 60 & 129.4 & \\
\hline & & & & & & Total Weight & 259.4 & \\
\hline
\end{tabular}

\begin{tabular}{|c|c|c|c|c|c|c|c|}
\hline Tank\# & Core \# & Matrix & Segment \# & Date & Jar/Vial \# & Vial Size (mLs) & Net Wt\# Found \\
\hline C-204 & & Sludge & Aug-23 & $5 / 17 / 96$ & 10115 & $\begin{array}{c}60 \\
\text { Total Weight }\end{array}$ & $\frac{65.1}{65.1}$ \\
\hline C-204 & R-7 & Sludge & Aug-022 & $5 / 20 / 96$ & 10119 & $\begin{array}{c}60 \\
\text { Total Weight }\end{array}$ & $\begin{array}{l}92 \\
92\end{array}$ \\
\hline
\end{tabular}


Vials in 11A1 and 11A2

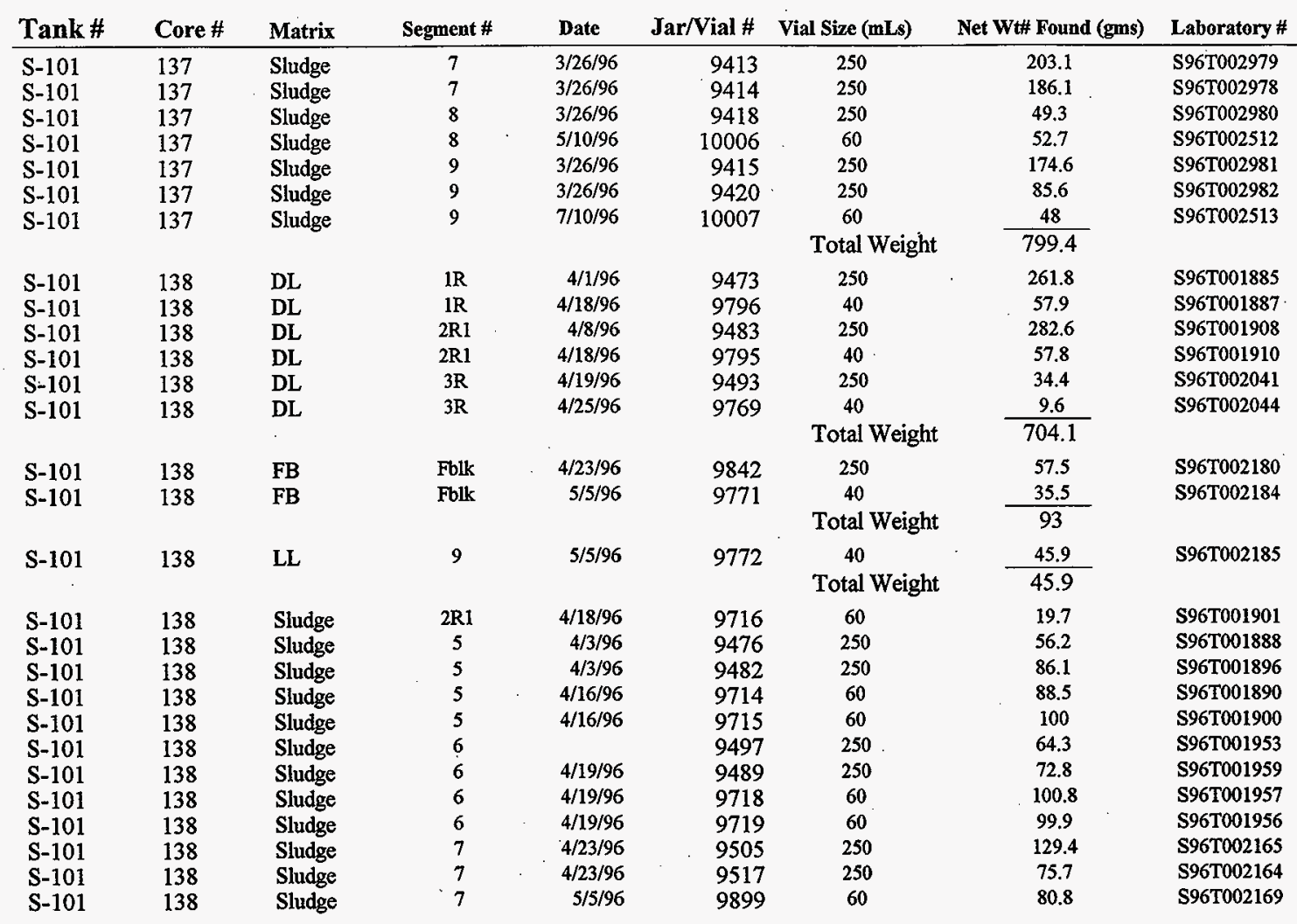


Vials in 11A1 and 11A2

\begin{tabular}{|c|c|c|c|c|c|c|c|c|}
\hline Tank \# & Core \# & Matrix & Segment \# & Date & Jar/Vial \# & Vial Size (mLs) & Net Wt\# Found (gms) & Laboratory \# \\
\hline S-101 & 138 & Sludge & 7 & $7 / 10 / 96$ & 9898 & 60 & 88.5 & S96T002168 \\
\hline S-101 & 138 & Sludge & 8 & $4 / 16 / 96$ & 9495 & 250 & 35.9 & S96T002002 \\
\hline$S=101$ & 138 & Sludge & 8 & $4 / 16 / 96$ & 9496 & 250 & 40 & S96T002003 \\
\hline S-101 & 138 & Sludge & 8 & $4 / 25 / 96$ & 9815 & 60 & 84.4 & \$96T002007 \\
\hline S-101 & 138 & Sludge & $8 \mathrm{UH}$ & $4 / 25 / 96$ & 9564 & 60 & 95.8 & S96T002006 \\
\hline S-101 & 138 & Sludge & 9 & $4 / 23 / 96$ & 9504 & 250 & 68.2 & S96T002174 \\
\hline S-101 & 138 & Sludge & 9 & $5 / 5 / 96$ & 9900 & 60 & 85.6 & S96T002179 \\
\hline S-101 & 138 & Sludge & 9 & $5 / 5 / 96$ & 9901 & 60 & 77.4 & S96T002178 \\
\hline \multirow[t]{2}{*}{$S-101$} & 138 & Sludge & Comp & $6 / 1 / 96$ & 10259 & 60 & 41.9 & S96T002799 \\
\hline & & & & & & Total Weight & 1591.9 & \\
\hline S-101 & 142 & Sludge & 1 & & 9494 & 250 & 212.2 & \$96T002057 \\
\hline S-101 & 142 & Sludge & 1 & $4 / 26 / 96$ & 9816 & 60 & 70.5 & S96T002079 \\
\hline S-101 & 142 & Sludge & 2 & $4 / 29 / 96$ & 9515 & 250 & 70.5 & S96T002208 \\
\hline S-101 & 142 & Sludge & 2 & $4 / 29 / 96$ & 9835 & 250 & 69.9 & S96T002299 \\
\hline S-101 & 142 & Sludge & 2 & $5 / 8 / 96$ & 9910 & 60 & 92.3 & S96T002306 \\
\hline S-101 & 142 & Sludge & 2 & $5 / 8 / 96$ & 9911 & 60 & 90.8 & S96T002307 \\
\hline S-101 & 142 & Sludge & 3 & & 9850 & 125 & 59.4 & S96T002301 \\
\hline S-101 & 142 & Sludge & 3 & $4 / 29 / 96$ & 9466 & 125 & 67.5 & S96T002300 \\
\hline S-101 & 142 & Sludge & 3 & $5 / 8 / 96$ & 9912 & 60 . & 89 & S96T002308 \\
\hline S-101 & 142 & Sludge & 3 & $5 / 8 / 96$ & 9913 & 60 & 92.6 & S96T002309 \\
\hline S-101 & 142 & Sludge & 4 & $4 / 19 / 96$ & 9508 & 250 & 110.7 & S96T002058 \\
\hline S-101 & 142 & Sludge & 4 & $4 / 26 / 96$ & 9503 & 250 & 87.5 & S96T002058 \\
\hline S-101 & 142 & Sludge & 4 & $4 / 26 / 96$ & 9817 & 60 & 75.8 & S96T002080 \\
\hline S-101 & 142 & Sludge & 4 & $4 / 26 / 96$ & 9818 & 60 & 71 & S96T002081 \\
\hline S-101 & 142 & Sludge & 5 & $4 / 19 / 96$ & 9502 & 250 & 120 & S96T002060 \\
\hline S-101 & 142 & Sludge & 5 & $4 / 19 / 96$ & 9511 & 250 & 100.8 & S96T002061 \\
\hline S-101 & 142 & Sludge & 5 & $4 / 26 / 96$ & 9819 & 60 & 72.9 & S96T002082 \\
\hline S-101 & 142 & Sludge & 5 & $4 / 26 / 96$ & 9874 & 60 & 71 & S96T002083 \\
\hline S-101 & 142 & Sludge & 6 & $4 / 19 / 96$ & 9512 & 250 & 128.3 & S96T002062 \\
\hline S-101 & 142 & Sludge & 6 & $4 / 19 / 96$ & 9513 & 250 & 97 & \$96T002063 \\
\hline S-101 & 142 & Sludge & 6 & $4 / 26 / 96$ & 9875 & 60 & 84.5 & S96T002084 \\
\hline S-101 & 142 & Sludge & 6 & $4 / 26 / 96$ & 9876 & 60 & 61.2 & S96T002085 \\
\hline S-101 & 142 & Sludge & 7 & $4 / 19 / 96$ & 9507 & 250 & 127.4 & S96T002064 \\
\hline S-101 & 142 & Sludge & 7 & $4 / 19 / 96$ & 9509 & 250 & 142.7 & S96T002065 \\
\hline
\end{tabular}

17-Mar-98 
Vials in 11A1 and 11A2

\begin{tabular}{|c|c|c|c|c|c|c|c|c|}
\hline Tank\# & Core\# & Matrix & Segment \# & Date & Jar/Vial \# & Vial Size (mLs) & Net Wt\# Found (gms) & Laboratory \# \\
\hline S-101 & 142 & Sludge & 7 & $4 / 26 / 96$ & 9877 & 60 & 84.7 & S96T002086 \\
\hline$S-101$ & 142 & Sludge & 7 & $4 / 26 / 96$ & 9878 & 60 & 82.9 & S96T002087 \\
\hline S-101 & 142 & Sludge & 8 & $4 / 19 / 96$ & 9471 & 125 & 27.9 & S96T002067 \\
\hline S-101 & 142 & Sludge & 8 & $4 / 19 / 96$ & 9501 & 250 & 129.2 & S96T002066 \\
\hline S-101 & 142 & Sludge & 8 & $4 / 26 / 96$ & 9879 & 60 & 60.5 & S96T002088 \\
\hline S-101 & 142 & Sludge & 8 & $4 / 26 / 96$ & 9880 & 60 & 75.2 & S96T002089 \\
\hline S- 101 & 142 . & Sludge & Comp & $6 / 1 / 96$ & 10260 & 60 & 71.1 & S $96 \mathrm{~T} 002730$ \\
\hline & & & & & & Total Weight & 2797 & \\
\hline
\end{tabular}

\begin{tabular}{|c|c|c|c|c|c|c|c|c|}
\hline Tank \# & Core \# & Matrix & Segment \# & Date & Jar/Vial \# & Vial Size (mLs) & Net Wt\# Found (gms) & Laboratory \# \\
\hline S-102 & 125 & DL & 7 & $2 / 27 / 97$ & 8433 & $\begin{array}{l}40 \\
\text { Total Weight }\end{array}$ & $\begin{array}{l}47.9 \\
47.9\end{array}$ & S96T000773 \\
\hline S-102 & 125 & FB & Fblk & $2 / 27 / 96$ & 8434 & $\begin{array}{l}40 \\
\text { Total Weight }\end{array}$ & $\begin{array}{r}41.5 \\
41.5\end{array}$ & S96T000841 \\
\hline S-102 & 125 & Salt & 11 & $1 / 24 / 96$ & 8868 & 250 & 149.6 & \$96T000592 \\
\hline S-102 & 125 & Salt & 11 & $2 / 28 / 96$ & 9277 & 60 & 92 & S96T000632 \\
\hline S-102 & 125 & Salt & 2 & $2 / 28 / 96$ & 9327 & 60 & 62.9 & S96T000751 \\
\hline S-102 & 125 & Salt & 3 & $2 / 28 / 96$ & 9328 & 60 & 94.4 & S96T000752 \\
\hline S-102 & 125 & Salt & 4 & $2 / 28 / 96$ & 9329 & 60 & 61.8 & S96T000753 \\
\hline S-102 & 125 & Salt & 4 & $2 / 28 / 96$ & 9330 & 60 & 13.9 & S96T000754 \\
\hline S-102 & 125 & Salt & 5 & $2 / 28 / 96$ & 9331 & 60 & 70.3 & S96T000619 \\
\hline S-102 & 125 & Salt & 5 & $2 / 28 / 96$ & 9332 & 60 & 22 & S96T000620 \\
\hline S- 102 & 125 & Salt & 6 & $1 / 23 / 96$ & 8877 & 250 & 41.6 & S96T000581 \\
\hline S-102 & 125 & Salt & 6 & $2 / 26 / 96$ & 9271 & 60 & 19.5 & S96T000621 \\
\hline S-102 & 125 & Salt & 6 & $2 / 26 / 96$ & 9283 & 60 & 23.9 & S96T000622 \\
\hline S-102 & 125 & Salt & 6 & $2 / 26 / 96$ & 9284 & 60 & 15.2 & S96T000763 \\
\hline S-102 & 125 & Salt & 7 & $2 / 27 / 96$ & 9286 & 60 & 68.6 & S96T000624 \\
\hline S-102 & 125 & Salt & 7 & $2 / 27 / 96$ & 9287 & 60 & 13 & S96T000625 \\
\hline S-102 & 125 & Salt & 8 & $2 / 27 / 96$ & 9169 & 60 & 57.1 & S96T000627 \\
\hline S-102 & 125 & Salt & 8 & $2 / 27 / 96$ & 9260 & 60 & 80.4 & S96T000626 \\
\hline $\mathrm{S}-102$ & 125 & Salt & Comp & $3 / 1 / 96$ & 9333 & 60 & 79.7 & S96T000786 \\
\hline & & & & & & Total Weight & 965.9 & \\
\hline
\end{tabular}


Vials in 11A1 and 11A2

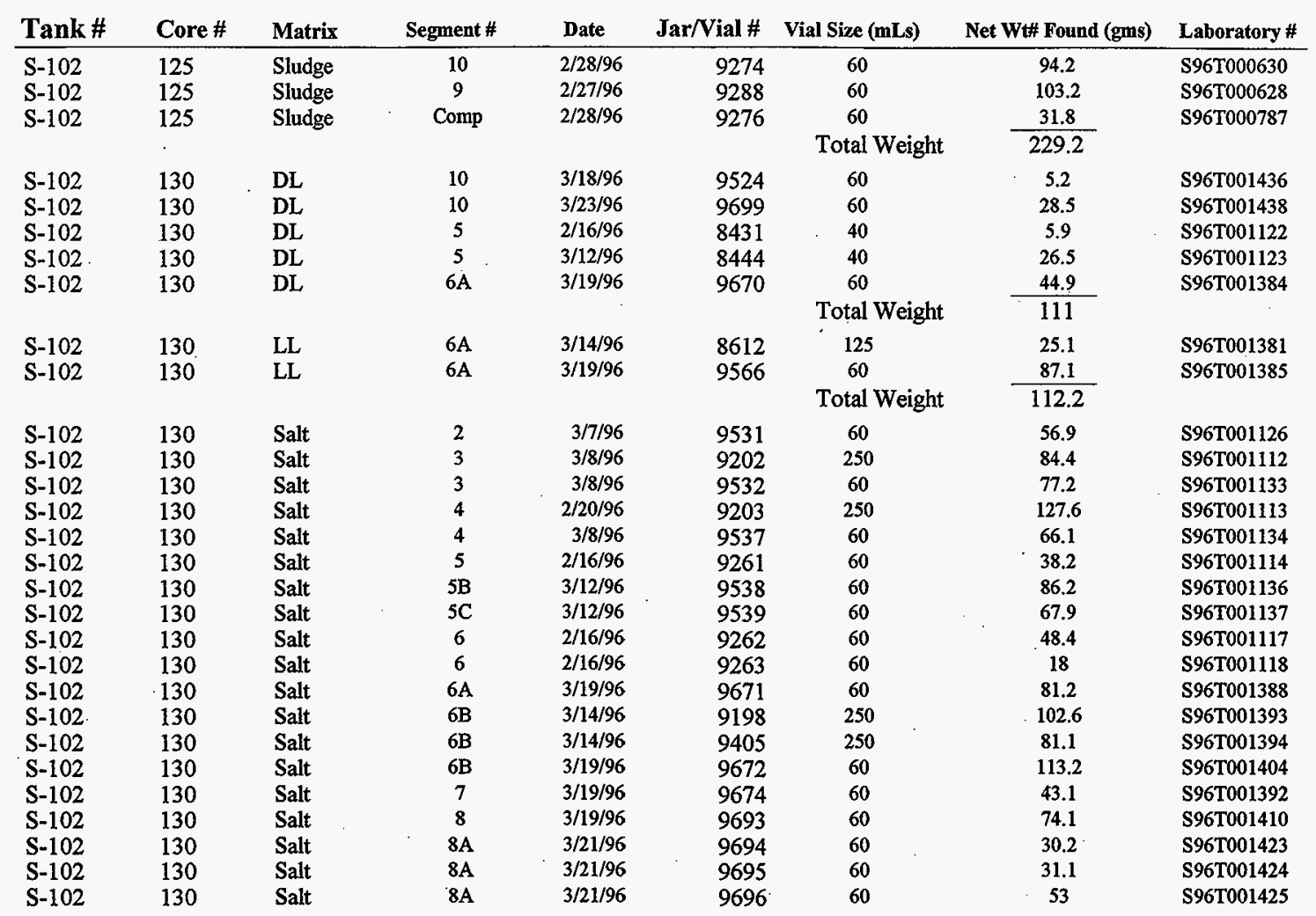


Vials in 11A1 and 11A2

\begin{tabular}{|c|c|c|c|c|c|c|c|c|}
\hline Tank \# & Core\# & Matrix & Segment \# & Date & Jar/Vial \# & Vial Size (mLs) & Net Wt\# Found (gms) & Laboratory \# \\
\hline & & & & & & Total Weight & 1280.5 & \\
\hline S-102 & 130 & Sludge & 10 & $3 / 18 / 96$ & 9407 & 250 & 62.9 & S96T001419 \\
\hline S-102 & 130 & Sludge & 10 & $3 / 18 / 96$ & 9409 & 250 & 53.2 & S96T001420 \\
\hline S-102 & 130 & Sludge & 11 & $3 / 18 / 96$ & 9536 & 60 & 16 & S96T001421 \\
\hline S- 102 & 130 & Sludge & 9 & $3 / 18 / 96$ & 9410 & 250 & 105.9 & S96T001439 \\
\hline S- 102 & 130 & Sludge & 9 & $3 / 18 / 96$ & 9412 & 250 & 106.9 & S96T001440 \\
\hline S-102 & 130 & Sludge & Comp & $4 / 9 / 96$ & 9705 & 60 & 73 & S96T001580 \\
\hline S-102 & 130 & Sludge & Comp & $4 / 10 / 96$ & 9706 & 60 & 56.8 & S96T001575 \\
\hline \multirow[t]{2}{*}{ S-102 } & 130 & Sludge & Comp & $3 / 18 / 97$ & 12116 & 60 & 19.3 & S97T000314 \\
\hline & & & & & & Total Weight & 494 & \\
\hline
\end{tabular}

\begin{tabular}{|c|c|c|c|c|c|c|c|c|}
\hline Tank \# & Core \# & Matrix & Segment \# & Date & Jar/Vial \# & Vial Size (mLs) & Net Wt\# Found (gms) & Laboratory \# \\
\hline S-104 & & Sludge & Comp & 9/8/97 & 12825 & $\begin{array}{c}250 \\
\text { Total Weight }\end{array}$ & $\begin{array}{r}149.6 \\
149.6\end{array}$ & \\
\hline
\end{tabular}

\begin{tabular}{|c|c|c|c|c|c|c|c|c|}
\hline Tank \# & Core\# & Matrix & Segment \# & Date & Jar/Vial \# & Vial Size (mLs) & Net Wt\# Found (gms) & Laboratory \# \\
\hline S-106 & 183 & DL & 1 & $2 / 27 / 97$ & 12173 & 250 & 316.2 & N/A \\
\hline S-106 & 183 & DL & 1 & $3 / 4 / 97$ & 12220 & 40 & 46.3 & \$97T000278 \\
\hline S-106 & 183 & $\mathrm{DL}$ & 3 & $2 / 27 / 97$ & 11821 & 125 & 33.4 & S97T000279 \\
\hline S-106 & 183 & $\overline{\mathrm{DL}}$ & 3 & $2 / 27 / 97$ & 12174 & 250 & 304.2 & N/A \\
\hline S-106 & 183 & DL & 3 & $3 / 5 / 97$ & 12217 & $40^{\circ}$ & 56.8 & \$97T000291 \\
\hline S-106 & 183 & $\mathrm{DL}$ & 4 & $2 / 28 / 97$ & 12175 & 250 & 212.1 & S97T000280 \\
\hline S-106 & 183 & $\mathrm{DL}$ & 4 & $3 / 4 / 97$ & 12216 & 40 & 55.9 & S97T000292 \\
\hline S-106 & 183 & DL & 5 & $2 / 28 / 97$ & 12176 & 250 & 221.6 & S97T000281 \\
\hline S-106 & 183 & DL & 5 & $3 / 5 / 97$ & 12219 & 40 & 57.5 & S97T000293 \\
\hline S-106 & 183 & $\mathrm{DL}$ & 7 & $2 / 28 / 97$ & 12177 & 250 & 177.5 & S97T000282 \\
\hline \multirow[t]{2}{*}{ S-106 } & 183 & DL & 7 & $3 / 4 / 97$ & 12218 & 40 & 52.7 & S97T000294 \\
\hline & & & & & - & Total Weight & 1534.2 & \\
\hline S-106 & 183 & Salt & 4 & $2 / 27 / 97$ & 12098 & 60 & 9.5 & S97T00030I \\
\hline S-106 & 183 & Salt & 5 & $3 / 5 / 97$ & 12099 & 60 & 18.2 & S97T000302 \\
\hline S-106 & 183 & Salt & 7 & $2 / 28 / 97$ & 12104 & 60 & 30.9 & S97T000303 \\
\hline
\end{tabular}


Vials in 11A1 and 11A2

\begin{tabular}{|c|c|c|c|c|c|c|c|c|}
\hline Tank \# & Core \# & Matrix & Segment \# & Date & Jar/Vial \# & Vial Size (mLs) & Net Wt\# Found (gms) & Laboratory \# \\
\hline S-106 & 183 & Salt & 8 & $3 / 11 / 97$ & 12110 & 60 & 64.5 & S97T000317 \\
\hline S-106 & 183 & Salt & 8 & $3 / 11 / 97$ & 12112 & 60 & 23.8 & S97T000327 \\
\hline \multirow[t]{2}{*}{ S-106 } & 183 & Salt & 9 & $3 / 11 / 97$ & 12111 & 60 & 39.8 & S97T000329 \\
\hline & & & & & & Total Weight & 186.7 & \\
\hline \multirow[t]{2}{*}{ S-106 } & 183 & Sludge & 9 & $3 / 11 / 97$ & 12108 & 60 & 49 & S97T000328 \\
\hline & & & & & & Total Weight & 49 & \\
\hline S-106 & 184 & DL & 1 & $3 / 6 / 97$ & 12178 & 250 & 182.9 & S97T000339 \\
\hline S-106 & 184 & DL & 1 & $3 / 12 / 97$ & 12214 & 40 & 58.4 & S97T000342 \\
\hline S-106 & 184 & DL & 2 & $3 / 6 / 97$ & 12179 & 250 & 170.4 & S97T000349 \\
\hline S-106 & 184 & DL & 2 & $3 / 12 / 97$ & 12224 & 40 & 57.8 & S97T000352 \\
\hline S-106 & 184 & DL & 6 & $4 / 4 / 97$ & 12192 & 250 & 93.7 & S97T000419 \\
\hline \multirow[t]{2}{*}{ S-106 } & 184 & DL & 6 & $4 / 4 / 97$ & 12225 & 40 & 58.5 & S97T000422 \\
\hline & & & & & & Total Weight & 621.7 & \\
\hline S-106 & 184 & FB & Fblk & $3 / 20 / 97$ & 12184 & 250 & 113 & S97T000403 \\
\hline \multirow[t]{2}{*}{ S-106 } & 184 & FB & Folk & $4 / 4 / 97$ & 12233 & 40 & 38.3 & \$97T000406 \\
\hline & & & & & & Total Weight & 151.3 & \\
\hline S-106 & 184 & LL & $6 \mathrm{R}$ & $3 / 20 / 97$ & 11877 & 125 & 112.6 & \\
\hline S-106 & 184 & LL & $6 \mathrm{R}$ & $3 / 20 / 97$ & 11879 & 125 & 16.4 & S97T000431 \\
\hline S-106 & 184 & LL & $6 \mathrm{RA}$ & $3 / 24 / 97$ & 12196 & 250 & 128.7 & S97T000446 \\
\hline \multirow[t]{2}{*}{ S-106 } & 184 & $\overline{\mathrm{LL}}$ & $6 \mathrm{RA}$ & $4 / 2 / 97$ & 12234 & 40 & 42.7 & S97T000448 \\
\hline & & & & & & Total Weight & 300.4 & \\
\hline S-106 & 184 & Salt & 2 & $3 / 12 / 97$ & 12109 & 60 & 60.3 & s97T000345 \\
\hline S-106 & 184 & Salt & 4 & $3 / 7 / 97$ & 11832 & 125 & 139.5 & GBR \\
\hline S-106 & 184 & Salt & 4 & $3 / 12 / 97$ & 12113 & 60 & 58.3 & S97T000355 \\
\hline S-106 & 184 & Salt & 4 & $3 / 12 / 97$ & 12115 & 60 & 40.3 & S97T000361 \\
\hline S-106 & 184 & Salt & 6 & $3 / 20 / 97$ & 12107 & 60 & 88.4 & S97T000415 \\
\hline S-106 & 184 & Salt & 6 & $3 / 20 / 97$ & 12303 & 60 & 42.9 & S97T000409 \\
\hline S-106 & 184 & Salt & $6 \mathrm{R}$ & $4 / 4 / 97$ & 12134 & 60 & 87.8 & S97T000427 \\
\hline \multirow[t]{2}{*}{ S-106 } & 184 & Salt & 6RA & $3 / 24 / 97$ & 12117 & 60 & 9.6 & S97T000440 \\
\hline & & & & & & Total Weight & 527.1 & \\
\hline S-106 & 187 & LL & IR & $3 / 26 / 97$ & 11874 & 125 & 21.8 & S97T001423 \\
\hline
\end{tabular}


Vials in 11A1 and 11A2

\begin{tabular}{|c|c|c|c|c|c|c|c|c|}
\hline Tank \# & Core\# & Matrix & Segment \# & Date & Jar/Vial \# & Vial Size (mLs) & Net Wt\# Found (gms) & Laboratory \# \\
\hline \multirow{3}{*}{ S-106 } & \multirow{3}{*}{187} & \multirow{3}{*}{ Salt } & \multirow{3}{*}{3} & \multirow{3}{*}{$3 / 27 / 97$} & \multirow{3}{*}{12136} & Total Weight & 21.8 & \multirow{3}{*}{ S97T001424 } \\
\hline & & & & & & 60 & 14.2 & \\
\hline & & & & & & Total Weight & 14.2 & \\
\hline Tank \# & Core\# & Matrix & Segment \# & Date & Jar/Vial \# & Vial Size (mLs) & Net Wt\# Found (gms) & Laboratory \# \\
\hline S-107 & 105 & DL & 1 & $10 / 2 / 95$ & 7979 & 40 & 39.5 & S95T002476 \\
\hline S-107 & 105 & DL & 2 & $9 / 20 / 95$ & 7936 & 250 & 65.7 & S95T002451 \\
\hline \multirow[t]{2}{*}{ S-107 } & \multirow[t]{2}{*}{105} & \multirow[t]{2}{*}{$\mathrm{DL}$} & \multirow[t]{2}{*}{2} & \multirow[t]{2}{*}{$10 / 4 / 95$} & \multirow[t]{2}{*}{7982} & 40 & 49.4 & \multirow[t]{2}{*}{ S95T002477 } \\
\hline & & & & & & Total Weight & 154.6 & \\
\hline S-107 & 105 & Sludge & 2 & $10 / 4 / 95$ & 7980 & 40 & 41.2 & S95T002479 \\
\hline S-107 & 105 & Sludge & 2 & $10 / 4 / 95$ & 7981 & 40 & 29 & S95T002478 \\
\hline S-107 & 105 & Sludge & 3 & $10 / 5 / 95$ & 7983 & 40 & 65.5 & S95T002537 \\
\hline S-107 & 105 & Sludge & 3 & $10 / 5 / 95$ & 7984 & 40 & 53.2 & \$95T002538 \\
\hline S-107 & 105 & Sludge & 3 & $9 / 15 / 97$ & 13156 & 60 & 15.4 & S95T002506 \\
\hline S-107 & 105 & Sludge & 4 & $10 / 5 / 95$ & 7985 & 40 & 64.5 & S95T002540 \\
\hline S-107 & 105 & Sludge & 4 & $10 / 5 / 95$ & 7986 & 40 & 65.8 & S95T002539 \\
\hline S-107 & 105 & Sludge & 4 & $9 / 11 / 97$ & 11839 & 125 & 107.1 & S95T002508 \\
\hline S-107 & 105 & Sludge & 4 & $9 / 16 / 97$ & 13162 & 60 & 76 & \$95T002507 \\
\hline S-107 & 105 & Sludge & 5 & & $7835 \mathrm{~A}$ & 60 & 44.2 & S95T002509 \\
\hline S-107 & 105 & Sludge & 5 & $9 / 22 / 95$ & 7836 & 250 & 76 & S95T002510 \\
\hline S-107 & 105 & Sludge & 5 & $10 / 5 / 95$ & 7987 & 40 & 61.2 & S95T002541 \\
\hline S-107 & 105 & Sludge & 5 & $10 / 5 / 95$ & 7988 & 40 & 61.9 & S95T002542 \\
\hline S-107 & 105 & Sludge & 6 & $10 / 5 / 95$ & 7989 & 40 & 57.3 & S95T002543 \\
\hline S-107 & 105 & Sludge & 6 & $10 / 5 / 95$ & 7990 & 40 & 82.2 & S95T002544 \\
\hline S-107 & 105 & Sludge & 7 & $10 / 5 / 95$ & 7991 & 40 & 64 & S95T002546 \\
\hline S-107 & 105 & Sludge & 7 & $10 / 5 / 95$ & 7992 & 40 & 41 & S95T002545 \\
\hline S-107 & 105 & Sludge & 8 & $10 / 5 / 95$ & 7993 & 40 & 8.9 & S95T002547 \\
\hline \multirow[t]{2}{*}{ S-107 } & \multirow[t]{2}{*}{105} & \multirow[t]{2}{*}{ Sludge } & \multirow[t]{2}{*}{8} & \multirow[t]{2}{*}{$10 / 5 / 95$} & \multirow[t]{2}{*}{7995} & 40 & 38.6 & \multirow[t]{2}{*}{ S95T002548 } \\
\hline & & & & & & Total Weight & 1053 & \\
\hline S-107 & 110 & $\mathrm{DL}$ & 1 & $4 / 12 / 96$ & 9792 & 40 & 9.5 & S96T002614 \\
\hline \multirow[t]{2}{*}{ S-107 } & \multirow[t]{2}{*}{110} & \multirow[t]{2}{*}{$\mathrm{DL}$} & \multirow[t]{2}{*}{2} & \multirow[t]{2}{*}{$10 / 6 / 95$} & \multirow[t]{2}{*}{7999} & 40 & 44.3 & \multirow[t]{2}{*}{ S95T002615 } \\
\hline & & & & & & Total Weight & 53.8 & \\
\hline
\end{tabular}


Vials in 11A1 and 11A2

\begin{tabular}{|c|c|c|c|c|c|c|c|c|}
\hline Tank \# & Core \# & Matrix & Segment \# & Date & Jar/Vial \# & Vial Size (mLs) & Net Wt\# Found (gms) & Laboratory \# \\
\hline S-107 & 110 & FB & Folk & $10 / 5 / 95$ & 7997 & $\begin{array}{c}40 \\
\text { Total Weight }\end{array}$ & $\frac{39.7}{39.7}$ & S95T002627 \\
\hline S-107 & 110 & Sludge & 2 & $10 / 6 / 95$ & 7998 & 40 & 59.4 & S95T002616 \\
\hline S-107 & 110 & Sludge & 2 & 9/9/97 & 13530 & 60 & 74.3 & S95T002586 \\
\hline S-107 & 110 & Sludge & 3 & 4/12/96. & 8456 & 40 & 25.9 & S96T002619 \\
\hline S-107 & 110 & Sludge & 3 & $4 / 15 / 96$ & 8452 & 40 & 39.2 & S96T002620 \\
\hline S-107 & 110 & Sludge & 3 & 9/9/97 & 13463 & 60 & 87.6 & S95T002588 \\
\hline S-107 & 110 & Sludge & 3 & $9 / 12 / 97$ & 13471 & 60 & 102.4 & S95T002590 \\
\hline S-107 & 110 & Sludge & 3 & $9 / 15 / 97$ & 13161 & 60 & 78 & S95T002587 \\
\hline S-107 & 110 & Sludge & 3 & $9 / 15 / 97$ & 13165 & 60 & 61.7 & S95T002589 \\
\hline S-107 & 110 & Sludge & $3 \mathrm{~A}$ & $10 / 6 / 95$ & 8001 & 40 & 53.9 & S95T002618 \\
\hline S-107 & 110 & Sludge & $3 \mathrm{~A}$ & $10 / 6 / 95$ & 8002 & 40 & 50 & S95T002617 \\
\hline S-107 & 110 & Sludge & 4 & $9 / 26 / 95$ & 7947 & 250 & 48 & S95T002592 \\
\hline S- 107 & 110 & Sludge & 4 & $10 / 9 / 95$ & 8006 & 40 & 66.9 & S95T002621 \\
\hline S-107 & 110 & Sludge & 4 & $10 / 9 / 95$ & 8007 & 40 & 51.8 & S95T002622 \\
\hline S-107 & 110 & Sludge & 4 & 9/9/97 & 13520 & 60 & 104.1 & S95T002591 \\
\hline S-107 & 110 & Sludge & 5 & $10 / 9 / 95$ & 8008 & 40 & 62.6 & S95T002623 \\
\hline S-107 & 110 & Sludge & 5 & $10 / 9 / 95$ & 8011 & 40 & 72.1 & S95T002624 \\
\hline S-107 & 110 & Sludge & 5 & $9 / 12 / 97$ & 11842 & 125 & 82 & S95T002613 \\
\hline S-107 & 110 & Sludge & 5 & $9 / 16 / 97$ & 13167 & 60 & 101.2 & S95T002612 \\
\hline S-107 & 110 & Sludge & 6 & 10/9/95 & 8009 & 40 & 60.5 & S95T002691 \\
\hline S-107 & 110 & Slidge & 6 & $10 / 9 / 95$ & 8010 & 40 & 76.7 & S95T002692 \\
\hline S-107 & 110 & Sludge & 6 & $9 / 29 / 97$ & 13450 & 60 & 107.8 & S95T002679 \\
\hline S-107 & 110 & Sludge & 7 & $10 / 9 / 95$ & 8012 & .40 & 56.4 & S95T002693 \\
\hline S-107 & 110 & Sludge & 7 & $4 / 15 / 96$ & 9793 & 60 & 132.9 & S96T002694 \\
\hline S-107 & 110 & Sludge & 8 & $10 / 10 / 95$ & 8017 & 40 & 50.4 & S95T002695 \\
\hline S-107 & 110 & Sludge & 8 & $10 / 10 / 95$ & 8018 & 40 & 58.6 & S95T002696 \\
\hline S-107 & 110 & Sludge & Comp & 11/1/95 & 8509 & 250 & 65.1 & S95T002917 \\
\hline & & & & & & Total Weight & 1829.5 & \\
\hline S-107 & 111 & DL & 1 & $9 / 29 / 95$ & 7957 & 250 & 135 & S95T002628 \\
\hline S-107 & 111 & DL & 1 & $10 / 10 / 95$ & 8014 & 40 & 38.9 & S95T002630 \\
\hline \multirow[t]{2}{*}{ S-107 } & 111 & $\mathrm{DL}$ & 2 & $10 / 10 / 95$ & 8015 & 40 & 47.7 & S95T002633 \\
\hline & & & & & & Total Weight & 221.6 & \\
\hline
\end{tabular}


Vials in 11A1 and 11A2

\begin{tabular}{|c|c|c|c|c|c|c|c|c|}
\hline Tank \# & Core \# & Matrix & Segment \# & Date & Jar/Vial \# & Vial Size (mLs) & Net Wt\# Found (gms) & Laboratory \# \\
\hline S-107 & 111 & Salt & 3 & $9 / 29 / 95$ & 7743 & 125 & 53 & S95T002641 \\
\hline S-107 & 111 & Salt & 3 & $10 / 10 / 95$ & 8021 & 40 & 58.4 & S95T002666 \\
\hline S-107 & 111 & Salt & 3 & $10 / 10 / 95$ & 8022 & 40 & 59.2 & S95T002667 \\
\hline S-107 & 111 & Salt & 3 & 7/9/96 & 10309 & 60 & 34.3 & S96T002640 \\
\hline S-107 & 111 & Salt & 4 & $10 / 11 / 95$ & 8019 & 40 & 72.6 & S95T002668 \\
\hline \multirow[t]{2}{*}{ S-107 } & \multirow[t]{2}{*}{111} & \multirow[t]{2}{*}{ Salt } & \multirow[t]{2}{*}{4} & \multirow[t]{2}{*}{$10 / 11 / 95$} & \multirow[t]{2}{*}{8020} & 40 & 73.5 & \multirow[t]{2}{*}{ S95T002669 } \\
\hline & & & & & & Total Weight & 351 & \\
\hline S-107 & 111 & Sludge & 2 & $10 / 10 / 95$ & 8016 & 40 & 65.7 & S95T002636 \\
\hline S-107 & 111 & Sludge & 2 & $7 / 9 / 96$ & 10308 & 60 & 91.4 & S96T002634 \\
\hline S-107 & 111 & Sludge & 4 & 9/26/97 & 13439 & 60 & 82.5 & S95T002642 \\
\hline S-107 & 111 & Sludge & 5 & $10 / 11 / 95$ & 8368 & 60 & 85 & S95T002670 \\
\hline S-107 & 111 & Sludge & 5 & $10 / 11 / 95^{\prime}$ & 8369 & 60 & 114.5 & S95T002671 \\
\hline S-107 & 111 & Sludge & 5 & $9 / 12 / 97$ & 13465 & 60 & 77.6 & S95T002645 \\
\hline S-107 & 111 & Sludge & 5 & $9 / 12 / 97$ & 13467 & 60 & 32.6 & S95T002644 \\
\hline S-107 & 111 & Sludge & 6 & $10 / 11 / 95$ & 8366 & 60 & 106.8 & S95T002672 \\
\hline S-107 & 111 & Sludge & 6 & $10 / 11 / 95$ & 8367 & 60 & 110.4 & S95T002673 \\
\hline S-107 & 111 & Sludge & 7 & $10 / 11 / 95$ & 8370 & 60 & 123.3 & S95T002674 \\
\hline S-107 & 111 & Sludge & 7 & $10 / 11 / 95$ & 8371 & 60 & 123.7 & S95T002675 \\
\hline S-107 & 111 & Sludge & 8 & $10 / 12 / 95$ & 8374 & 60 & 33.3 & S95T002676 \\
\hline \multirow[t]{2}{*}{ S-107 } & \multirow[t]{2}{*}{111} & \multirow[t]{2}{*}{ Sludge } & \multirow[t]{2}{*}{ Comp } & \multirow[t]{2}{*}{$11 / 1 / 95$} & \multirow[t]{2}{*}{8505} & 250 & 98.1 & \multirow[t]{2}{*}{ S95T002918 } \\
\hline & & & & & & Total Weight & 1144.9 & \\
\hline Tank\# & Core\# & Matrix & Segment \# & Date & Jar/Vial \# & Vial Size (mLs) & Net Wt\# Found (gms) & Laboratory \# \\
\hline S-109 & 158 & LL & 2 & $7 / 16 / 96$ & 10510 & 60 & 473.7 & S96T003754 \\
\hline S-109 & 158 & LL & 3 & $7 / 19 / 96$ & 10418 & 125 & 34.3 & S96T003932 \\
\hline S-109 & 158 & LL & 3 & $7 / 23 / 96$ & 10680 & 60 & 60 & S96T003934 \\
\hline & & & & & & Total Weight & 568 & \\
\hline S-109 & 158 & Salt & 1 & $7 / 23 / 96$ & 10678 & 60 & 89.6 & S96T003928 \\
\hline S-109 & 158 & Salt & 1 & $7 / 23 / 96$ & 10679 & 60 & 34.7 & S96T003929 \\
\hline S-109 & 158 & Salt & 2 & $7 / 16 / 96$ & 10514 & 60 & 156.4 & S96T003733 \\
\hline S-109 & 158 & Salt & $2 \mathrm{ALH}$ & $7 / 24 / 96$ & 10674 & 60 & 12.9 & S96T004016 \\
\hline S. 109 & 158 & Salt & $2 \mathrm{~B}$ & $7 / 16 / 96$ & 10507 & 60 & 19.4 & S96T003757 \\
\hline
\end{tabular}


Vials in 11A1 and 11A2

\begin{tabular}{|c|c|c|c|c|c|c|c|c|}
\hline Tank \# & Core \# & Matrix & Segment \# & Date & Jar/Vial \# & Vial Size (mLs) & Net Wt\# Found (gms) & Laboratory \# \\
\hline S-109 & 158 & Salt & $2 B$ & $7 / 16 / 96$ & 10509 & 60 & 11.3 & S96T003758 \\
\hline S-109 & 158 & Salt & 3 & $7 / 19 / 96$ & 10639 & 60 & 47.4 & S96T003918 \\
\hline S-109 & 158 & Salt & 3 & $7 / 19 / 96$ & 10640 & 60 & 26.3 & S96T003919 \\
\hline S-109 & 158 & Salt & $3 \mathrm{~A}$ & $7 / 23 / 96$ & 10683 & 60 & 76.5 & S96T003930 \\
\hline S-109 & 158 & Salt & 4 & $7 / 23 / 96$ & 10684 & $\begin{array}{c}60 \\
\text { Total Weight }\end{array}$ & $\begin{array}{c}59.8 \\
534.3\end{array}$ & S96T003931 \\
\hline S-109 & 160 & DL & $2 \mathrm{C}$ & $7 / 22 / 96$ & 10677 & 60 & 40.6 & S96T004031 \\
\hline S-109 & 160 & $\overline{\mathrm{DL}}$ & $2 \mathrm{C}$ & $7 / 30 / 96$ & 10778 & $\begin{array}{c}60 \\
\text { Total Weight }\end{array}$ & $\frac{18.3}{58.9}$ & S96T004032 \\
\hline $\begin{array}{l}\text { S-109 } \\
\text { S-109 }\end{array}$ & $\begin{array}{l}160 \\
160\end{array}$ & $\begin{array}{l}\text { FB } \\
\text { FB }\end{array}$ & $\begin{array}{l}\text { Fblk } \\
\text { Fblk }\end{array}$ & $\begin{array}{l}7 / 30 / 96 \\
7 / 30 / 96\end{array}$ & $\begin{array}{l}10517 \\
10779\end{array}$ & $\begin{array}{c}250 \\
60 \\
\text { Total Weight }\end{array}$ & $\begin{array}{c}161.1 \\
54.8 \\
215.9\end{array}$ & $\begin{array}{l}\text { S96T004022 } \\
\text { S96T004024 }\end{array}$ \\
\hline S-109 & 160 & LL & $2 \mathrm{~A}$ & $7 / 16 / 96$ & 9925 & 40 & 9.1 & S96T003824 \\
\hline S-109 & 160 & LL & $2 B$ & $7 / 28 / 96$ & 10784 & 60 & 58.5 & S96T004027 \\
\hline S-109 & 160 & $\overline{\mathrm{LL}}$ & $2 B$ & $7 / 30 / 96$ & 10416 & $\begin{array}{l}125 \\
\text { Total Weight }\end{array}$ & $\frac{50.9}{118.5}$ & S96T004025 \\
\hline S-109 & 160 & Salt & 1 & $7 / 20 / 96$ & 10337 & 60 & 36.2 & S96T003809 \\
\hline S-109 & 160 & Salt & 2 & $7 / 20 / 96$ & 10641 & 60 & 49.1 & S96T003813 \\
\hline S-109 & 160 & Salt & $2 \mathrm{~B}$ & $7 / 30 / 96$ & 10675 & 60 & 20 & S96T004029 \\
\hline S-109 & 160 & Salt & $2 \mathrm{~B}$ & $7 / 30 / 96$ & 10676 & 60 & 10.6 & S96T004028 \\
\hline & & & & & & Total Weight & 115.9 & \\
\hline
\end{tabular}

\begin{tabular}{|c|c|c|c|c|c|c|c|c|}
\hline Tank\# & Core \# & Matrix & Segment \# & Date & Jar/Vial \# & Vial Size (mLs) & Net Wt\# Found (gms) & Laboratory \# \\
\hline$S-110$ & 140 & LL & 4 & $4 / 22 / 96$ & 9457 & 125 & 40.6 & \$96T002352 \\
\hline S-110 & 140 & LL & 4 & $7 / 16 / 96$ & 9773 & 40 & 40.3 & S96T002372 \\
\hline S- 110 & 140 & LL & $4 A$ & & $9774 \mathrm{~A}$ & & 36.2 & S96T002374 \\
\hline S-110 & 140 & LL & $4 \mathrm{~A}$ & $7 / 16 / 96$ & 9774 & 40 & 39.2 & S96T002374 \\
\hline & & & & & & Total Weight & 156.3 & \\
\hline S-110 & 140 & Salt & 1 & $5 / 6 / 96$ & 9902 & 60 & $60: 9$ & S96T002363 \\
\hline S-110 & 140 & Salt & 1 & $5 / 6 / 96$ & 9903 & 60 & 114.9 & S96T002364 \\
\hline S-110 & 140 & Salt & 2 & $4 / 23 / 96$ & 9516 & 250 & 110.6 & S96T002210 \\
\hline
\end{tabular}


Vials in 11A1 and 11A2

\begin{tabular}{|c|c|c|c|c|c|c|c|c|}
\hline Tank \# & Core \# & Matrix & Segment \# & Date & Jar/Vial \# & Vial Size (mLs) & Net Wt\# Found (gms) & Laboratory \# \\
\hline S-1 10 & 140 & Salt & 2 & $5 / 6 / 96$ & 9904 & 60 & 107.6 & S96T002365 \\
\hline S-110 & 140 & Salt & 2 & $5 / 6 / 96$ & 9905 & 60 & 115.2 & S96T002366 \\
\hline$S-110$ & 140 & Salt & 3 & $4 / 22 / 96$ & 9506 & 250 & 104 & S96T002211 \\
\hline$S-110$ & 140 & Salt & 3 & $4 / 22 / 96$ & 9514 & 250 & 101.5 & S96T002212 \\
\hline$S-110$ & 140 & Salt & 3 & $5 / 6 / 96$ & 9906 & 60 & 115.2 & S96T002367 \\
\hline S- 110 & 140 & Salt & 3 & $5 / 6 / 96$ & 9907 & 60 & 115.5 & S96T002368 \\
\hline S-110 & 140 & Salt & 4 & $5 / 7 / 96$ & 9908 & 60 & 27.3 & S96T002369 \\
\hline S-110 & 140 & Salt & $4 \mathrm{~A}$ & $5 / 7 / 96$ & 9909 & 60 & 46 & S96T002370 \\
\hline S-110 & 140 & Salt & 4B & $4 / 24 / 96$ & 9809 & 60 & 64.1 & S96T002371 \\
\hline$S-110$ & 140 & Salt & $4 \mathrm{C}$ & $5 / 21 / 96$ & 10202 & 60 & 23.2 & S96T003100 \\
\hline$S-110$ & 140 & Salt & $4 \mathrm{C}$ & $7 / 15 / 96$ & 9776 & 40 & 45.9 & S96T003108 \\
\hline \multirow[t]{2}{*}{ S-110 } & 140 & Salt & $4 \mathrm{C}$ & $7 / 15 / 96$ & 9777 & 40 & 56.8 & S96T003111 \\
\hline & & & & & & Total Weight & 1208.7 & \\
\hline S-110 & Grab & GL & 1 & $8 / 29 / 97$ & 13236 & 40 & 59 & na \\
\hline \multirow[t]{2}{*}{ S-110 } & Grab & GL & 2 & $8 / 29 / 97$ & 13237 & 40 & 60.3 & na \\
\hline & & & & & & Total Weight & 119.3 & \\
\hline
\end{tabular}

\begin{tabular}{|c|c|c|c|c|c|c|c|c|}
\hline Tank \# & Core \# & Matrix & Segment \# & Date & Jar/Vial \# & Vial Size (mLs) & Net Wt\# Found (gms) & Laboratory \# \\
\hline $\begin{array}{l}\text { S-111 } \\
\text { S-111 }\end{array}$ & $\begin{array}{l}149 \\
149\end{array}$ & $\begin{array}{l}\mathrm{DL} \\
\mathrm{DL}\end{array}$ & $\begin{array}{l}1 \\
1\end{array}$ & $\begin{array}{l}5 / 28 / 96 \\
7 / 15 / 96\end{array}$ & $\begin{array}{r}10216 \\
9932\end{array}$ & $\begin{array}{l}250 \\
40\end{array}$ & $\begin{array}{l}257.5 \\
31.8\end{array}$ & $\begin{array}{l}\text { S96T003310 } \\
\text { S96T003364 }\end{array}$ \\
\hline S-111 & 149 & DL & 2 & $4 / 28 / 96$ & $\begin{array}{l}7932 \\
10198\end{array}$ & 250 & 349.3 & S96T003364 \\
\hline S-111 & 149 & $\mathrm{DL}$ & 2 & $7 / 15 / 96$ & 9933 & 40 & 17.7 & S96T003366 \\
\hline S-111 & 149 & $\mathrm{DL}$ & 3 & $5 / 28 / 96$ & 10214 & 250 & 339.7 & \\
\hline \multirow[t]{2}{*}{ S-111 } & 149 & $\mathrm{DL}$ & 3 & $7 / 15 / 96$ & 9934 & 40 & 42.3 & S96T003367 \\
\hline & & & & & & Total Weight & 1038.3 & \\
\hline S-111 & 149 & FB & Fodk & $6 / 5 / 96$ & 10226 & 250 & 190.8 & S96T003342 \\
\hline \multirow[t]{2}{*}{ S-111 } & 149 & FB & Folk & $7 / 15 / 96$ & 9923 & 40 & 40.2 & S96T003368 \\
\hline & & & & & & Total Weight & 231 & \\
\hline S-111 & 149 & Salt & 10 & $6 / 17 / 96$ & 10240 & 250 & 186.9 & S96T003613 \\
\hline S-111 & 149 & Salt & 10 & $7 / 18 / 96$ & 10636 & 60 & 89.2 & S96T003618 \\
\hline S-111 & 149 & Salt & 11 & & 10241 & 250 & 70.3 & S96T003345 \\
\hline S-111 & 149 & Salt & 11 & $7 / 18 / 96$ & 10637 & 60 & 85 & S96T003382 \\
\hline
\end{tabular}


Vials in 11A1 and 11A2

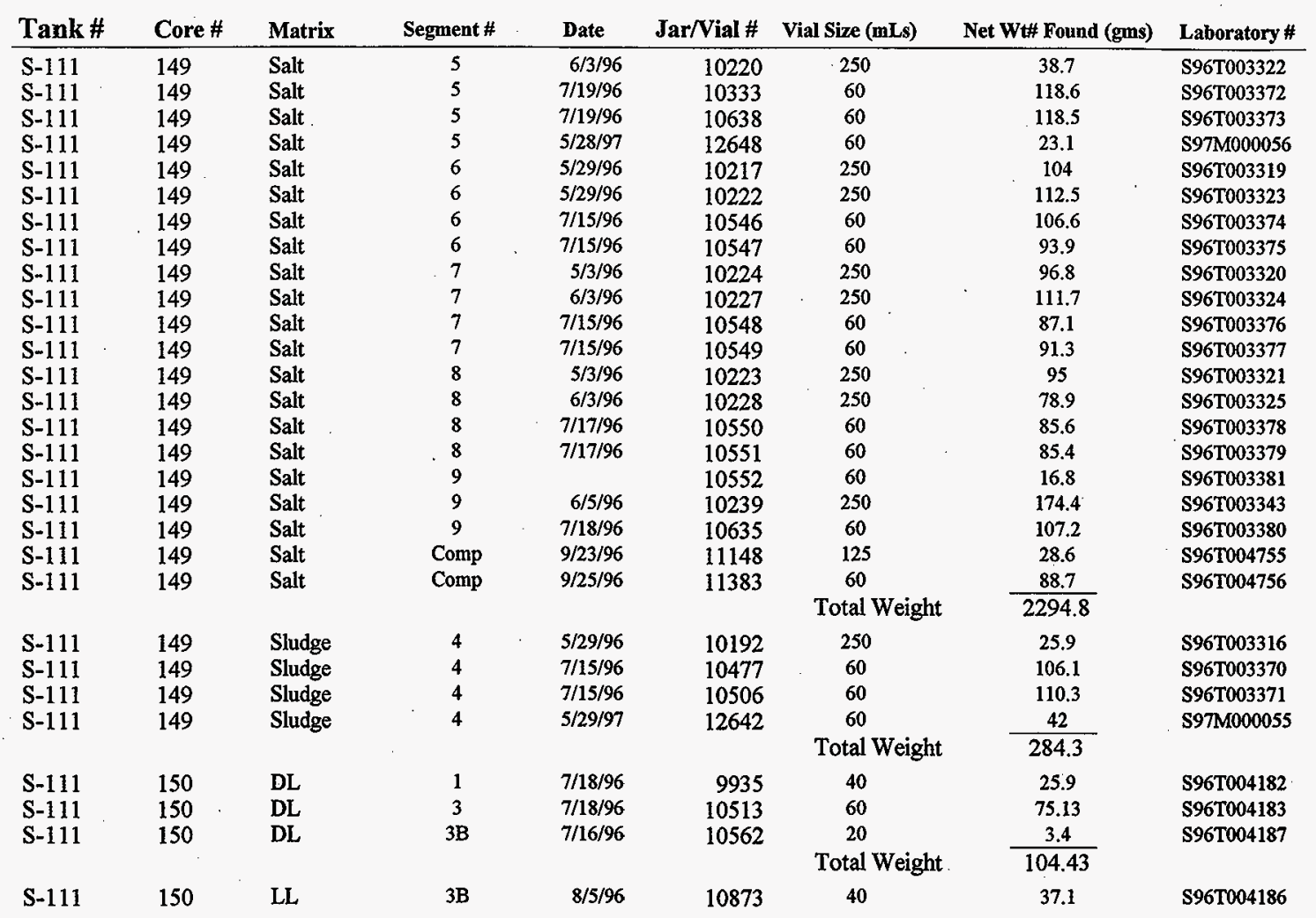


Vials in 11A1 and 11A2

\begin{tabular}{|c|c|c|c|c|c|c|c|c|}
\hline Tank \# & Core\# & Matrix & Segment \# & Date & Jar/Vial \# & Vial Size (mLs) & Net Wt\# Found (gms) & Laboratory \# \\
\hline & & & & & & Total Weight & 37.1 & \\
\hline S-111 & 150 & Salt & 1 & $7 / 18 / 96$ & 10512 & 60 & 18.1 & S96T004189 \\
\hline S-111 & 150 & Salt & 2 & $7 / 18 / 96$ & 10422 & 125 & 98.3 & S96T004195 \\
\hline S-111 & 150 & Salt & 2 & $7 / 18 / 96$ & 10525 & 250 & 81.2 & S96T004196 \\
\hline S-111 & 150 & Salt & 2 & $8 / 5 / 96$ & 10843 & 60 & 86.8 & S96T004190 \\
\hline S-111 & 150 & Salt & 2 & $8 / 5 / 96$ & 10844 & 60 & 95.5 & S96T004191 \\
\hline S-111 & 150 & Salt & 3 & $7 / 18 / 96$ & 10642 & 60 & 114.4 & \$96T004192 \\
\hline S-111 & 150 & Salt & $3 A$ & $8 / 5 / 96$ & 10859 & 40 & 34.8 & \$96T004185 \\
\hline S-111 & 150 & Salt & $3 \mathrm{~A}$ & $8 / 5 / 96$ & 10860 & 40 & 60.4 & S96T004184 \\
\hline S-111 & 150 & Salt & $3 B$ & $7 / 16 / 96$ & 10586 & 20 & 7.4 & S96T004193 \\
\hline S-111 & 150 & Salt & $3 \mathrm{C}$ & $7 / 16 / 96$ & 10511 & 60 & 23.8 & S96T004194 \\
\hline \multirow[t]{3}{*}{ S-111 } & 150 & Salt & $3 \mathrm{C}$ & $8 / 5 / 96$ & 10861 & 40 & 35.4 & S96T004188 \\
\hline & & & & & & Total Weight & 656.1 & \\
\hline & & & & & - & & & \\
\hline Tank \# & Core \# & Matrix & Segment \# & Date & J̦ar/Vial \# & Vial Size (mLs) & Net Wt\# Found (gms) & Laboratory \# \\
\hline S-151 & Grab & GL & & $1 / 30 / 96$ & 9215 & 60 & 40.7 & S95T003121 \\
\hline S-151 & Grab &. $\mathrm{GL}$ & & $1 / 30 / 96$ & 9216 & 60 & 40.8 & S95T003122 \\
\hline S-151 & Grab & GL & & $1 / 30 / 96$ & 9217 & 60 & 57.8 & S95T003200 \\
\hline & & & & & & Total Weight & 139.3 & \\
\hline Tank \# & Core\# & Matrix & Segment \# & Date & Jar/Vial \# & Vial Size (mLs) & Net Wt\# Found (gms) & Laboratory \# \\
\hline SX-101 & 225 & DL & 1 & $12 / 16 / 97$ & 12920 & 250 & 153.9 & S97T002409 \\
\hline SX-101 & 225 & DL & 2 & $12 / 16 / 97$ & 12921 & 250 & 96.6 & S97T002419 \\
\hline SX-101 & 225 & DL & 2 & $12 / 22 / 97$ & 13875 & 40 & 61.2 & S97T002425 \\
\hline SX-101 & 225 & $\mathrm{DL}$ & 3 & $12 / 16 / 97$ & 11852 & 125 & 35.8 & S97T002418 \\
\hline SX-101 & 225 & DL & 3 & $12 / 16 / 97$ & 12926 & 250 & 359.7 & \\
\hline SX-101 & 225 & $\mathrm{DL}$ & 3 & $12 / 19 / 97$ & 13651 & 60 & 37.9 & S97T002424 \\
\hline SX-101 & 225 & $\mathrm{DL}$ & $3 \mathrm{~A}$ & $12 / 18 / 97$ & 12925 & 250 & 131.6 & S97T002442 \\
\hline SX-101 & 225 & $\mathrm{DL}$ & $3 \mathrm{~A}$ & $12 / 22 / 97$ & 13877 & 40 & 60.2 & S97T002445 \\
\hline SX-101 & 225 & DL & 5 & $12 / 18 / 97$ & 12927 & 250 & 210.1 & S97T002471 \\
\hline SX-101 & 225 & $\mathrm{DL}$ & 5 & $12 / 22 / 97$ & 13874 & 40 & 62.1 & S97T002479 \\
\hline SX-101 & 225 & $\mathrm{DL}$ & $S R$ & $12 / 11 / 97$ & 12923 & 250 & 214.4 & S97T002470 \\
\hline
\end{tabular}

17-Mar-98

Page 81 of 114 
Vials in 11A1 and 11A2

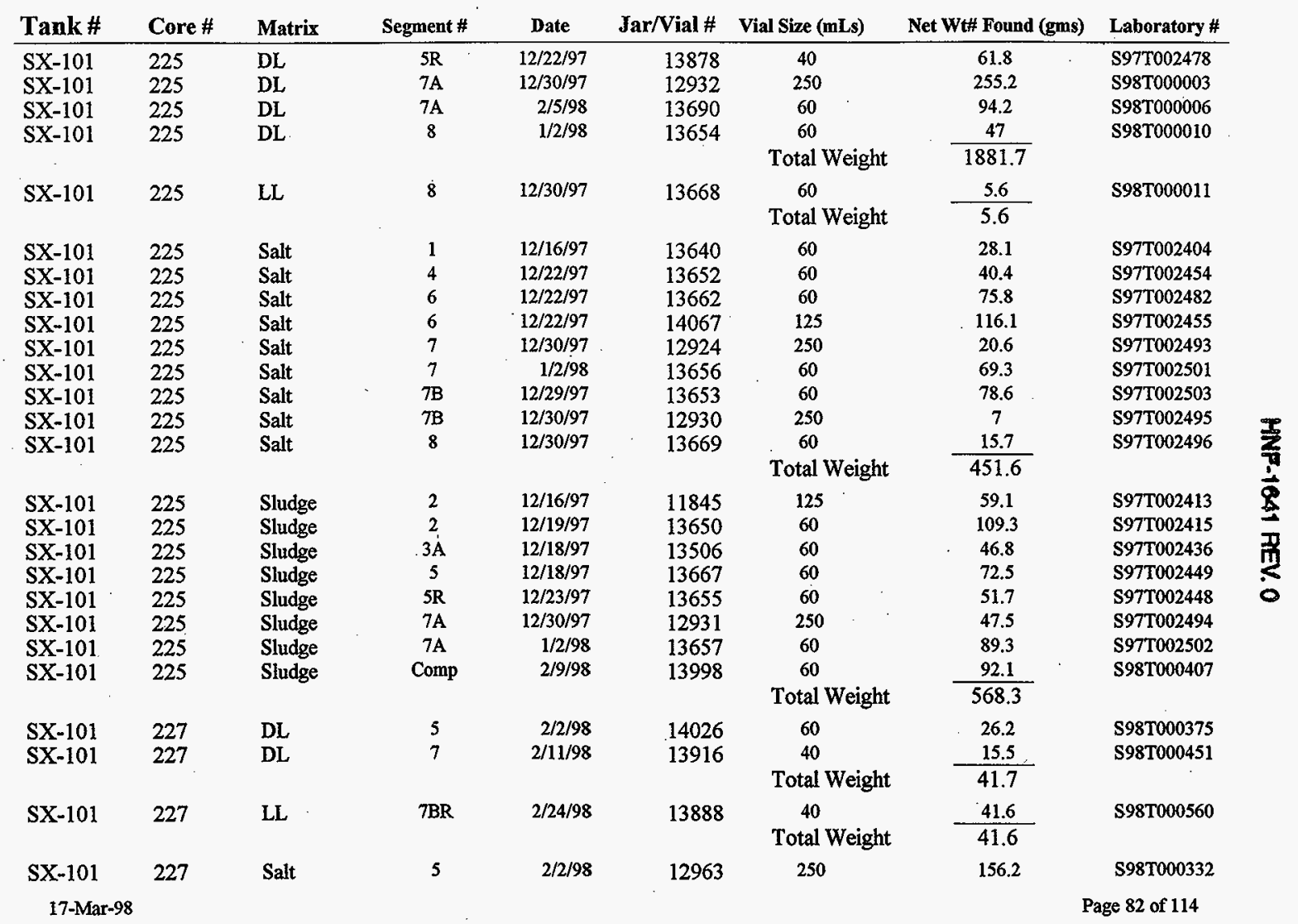


Vials in 11A1 and 11A2

\begin{tabular}{|c|c|c|c|c|c|c|c|c|}
\hline Tank \# & Core \# & Matrix & Segment \# & Date & Jar/Vial \# & Vial Size (mLs) & Net Wt\# Found (gms) & Laboratory \# \\
\hline SX-101 & 227 & Salt & 5 & $2 / 6 / 98$ & 14001 & 60 & 124.2 & S98T000354 \\
\hline SX-101 & 227 & Salt & $5 \mathrm{~A}$ & $2 / 2 / 98$ & 12957 & 250 & 82.1 & S98T000333 \\
\hline$S X-101$ & 227 . & Salt & $5 \mathrm{~A}$ & $2 / 2 / 98$ & 12973 & 250 & 73.5 & S98T000334 \\
\hline SX-101 & 227 & Salt & $5 \mathrm{~A}$ & $2 / 12 / 98$ & 13999 & 60 & 53.1 & S98T000452 \\
\hline SX-101 & 227 & Salt & $5 \mathrm{~A}$ & $2 / 12 / 98$ & 14000 & 60 & 48.8 & S98T000458 \\
\hline SX-101 & 227 & Salt & $5 A$ & $2 / 12 / 98$ & 14006 & 60 & 80 & S98T000355 \\
\hline SX-101 & 227 & Salt & $5 A$ & $2 / 12 / 98$ & 14007 & 60 & 86.5 & S98T000356 \\
\hline SX-101 & 227 & Salt & 6 & $2 / 5 / 98$ & 12965 & 250 & 126.2 & \$98T000431 \\
\hline SX-101 & 227 & Salt & 6 & $2 / 5 / 98$ & 12966 & 250 & 40.5 & S98T000430 \\
\hline SX-101 & 227 & Salt & 6 & $2 / 11 / 98$ & 14002 & 60 & 50.1 & S98T000459 \\
\hline SX-101 & 227 & Salt & 6 & $2 / 11 / 98$ & 14003 & 60 & 34.8 & S98T000460 \\
\hline SX-101 & 227 & Salt & 6 & $2 / 11 / 98$ & 14005 & 60 & 79.9 & S98T000436 \\
\hline$S X-101$ & 227 & Salt & 6 & $2 / 11 / 98$ & 14008 & 60 & 93.8 & S98T000437 \\
\hline SX-101 & 227 & Salt & 7 & $2 / 11 / 98$ & 14009 & 60 & 53 & S98T000438 \\
\hline SX-101 & 227 & Salt & 7 & $2 / 12 / 98$ & 14004 & 60 & 11 & S98T000461 \\
\hline \multirow[t]{2}{*}{ SX-101 } & 227 & Salt & $7 \mathrm{~A}$ & $2 / 19 / 98$ & 14030 & 60 & 37.7 & S98T000557 \\
\hline & & & & & & Total Weight & 1231.4 & \\
\hline SX-101 & 227 & Sludge & 1 & $2 / 2 / 98$ & 13636 & 60 & 77.9 & S98T000336 \\
\hline SX-101 & 227 & Sludge & 1 & $2 / 2 / 98$ & 13641 & 60 & 83.6 & S98T000339 \\
\hline SX-101 & 227 & Sludge & 2 & $2 / 2 / 98$ & 13635 & 60 & 69.5 & S98T000352 \\
\hline \multirow[t]{2}{*}{ SX-101 } & 227 & Sludge & 4 & $2 / 2 / 98$ & 14023 & 60 & 47 & S98T000353 \\
\hline & & & & . & & Total Weight & 278 & \\
\hline Tank\# & Core\# & Matrix & Segment \# & Date & Jar/Vial \# & Vial Size (mLs) & Net Wt\# Found (gms) & Laboratory \# \\
\hline SX-103 & Grab & GL & $97-1$ & $6 / 17 / 97$ & 12727 & 60 & 73.9 & S97T001405 \\
\hline SX-103 & Grab & GL & $97-2$ & $6 / 19 / 97$ & 12723 & 60 & 56.9 & S97T001406 \\
\hline SX-103 & Grab & GL & $97-2$ & $6 / 19 / 97$ & 12724 & 60 & 40.8 & S97T001407 \\
\hline SX-103 & Grab & GL & $97-3$ & $6 / 18 / 97$ & 12728 & 60 & 6 & S97T001416 \\
\hline \multirow[t]{2}{*}{ SX-103 } & Grab & GL & $97-3$ & $6 / 18 / 97$ & 12729 & 60 & 96.4 & S97T001410 \\
\hline & & & & & & Total Weight & 274 & \\
\hline
\end{tabular}


Vials in 11A1 and 11A2

\begin{tabular}{|c|c|c|c|c|c|c|c|c|}
\hline Tank \# & Core \# & Matrix & Segment \# & Date & Jar/Vial \# & Vial Size (mLs) & Net Wt\# Found (gms) & Laboratory \# \\
\hline SX-104 & grab & GL & $97-1$ & & $4 \mathrm{SX} 971$ & 125 & 181.9 & \\
\hline SX-104 & grab & GL & $97-1$ & $2 / 11 / 98$ & 14010 & 60 & 4.8 & S98T000428 \\
\hline SX-104 & grab & GL & $97-1$ & $2 / 12 / 98$ & 13562 & 125 & 109 & S98T000429 \\
\hline SX-104 & grab & GL & $97-2$ & $5 / 15 / 97$ & 11833 & 125 & 136.5 & S97T000867 \\
\hline SX-104 & grab & GL & $97-2$ & $5 / 15 / 97$ & 12335 & 60 & 23.6 & S97T000866 \\
\hline & & & & & & Total Weight & 455.8 & \\
\hline
\end{tabular}

\begin{tabular}{|c|c|c|c|c|c|c|c|c|c|}
\hline Tank \# & Core \# & Matrix & Segment \# & Date & Jar/Vial \# & Vial Size (mLs) & \multicolumn{2}{|c|}{ Net Wt\# Found (gms) } & Laboratory \# \\
\hline SX-106 & 223 & $\mathrm{DL}$ & 1 & $10 / 20 / 97$ & 12909 & 250 & 239.5 & & S97T002180 \\
\hline SX-106 & 223 & $\mathrm{DL}$ & 1 & $10 / 22 / 97$ & 13865 & 40 & 48.5 & & S97T002181 \\
\hline SX-106 & 223 & DL & 2 & $10 / 20 / 97$ & 12996 & 250 & 134.7 & & S97T002187 \\
\hline SX-106 & 223 & $\mathrm{DL}$ & 2 & $10 / 22 / 97$ & 11858 & 125 & 149 & & S97T002189 \\
\hline SX-106 & 223 & DL & 3 & $10 / 20 / 97$ & 12910 & 250 & 221.3 & & S97T002197 \\
\hline SX-106 & 223 & $\mathrm{DL}$ & 3 & $10 / 22 / 97$ & 13866 & 40 & 59.3 & . & S97T002201 \\
\hline SX-106 & 223 & $\mathrm{DL}$ & 4 & $10 / 21 / 97$ & 13537 & 250 & 265.6 & & S97T002198 \\
\hline SX-106 & 223 & DL & 4 & $10 / 22 / 97$ & 13270 & 40 & 58.4 & & S97T002202 \\
\hline SX-106 & 223 & $\overline{D L}$ & 5 & $10 / 30 / 97$ & 12894 & 250 & 184.2 & & S97T002214 \\
\hline SX-106 & 223 & $\overline{D L}$ & 5 & $10 / 31 / 97$ & 13502 & 60 & 87 & & S97T002216 \\
\hline & & & & & & Total Weight & 1447.5 & & \\
\hline SX-106 & 223 & Salt & 1 & $10 / 20 / 97$ & 13498 & 60 & 25.1 & & S97T002175 \\
\hline SX-106 & 223 & Salt & 10 & $11 / 10 / 97$ & 13541 & 250 & 98.9 & & S97T002260 \\
\hline SX-106 & 223 & Salt & 2 & $10 / 20 / 97$ & 11864 & 125 & 42.7 & & S97T002182 \\
\hline SX-106 & 223 & Salt & 3 & $10 / 20 / 97$ & 11867 & 125 & 41.1 & & S97T002190 \\
\hline SX-106 & 223 & Salt & 4 & $10 / 21 / 97$ & 13494 & 60 & 21.3 & & S97T002203 \\
\hline SX-106 & 223 & Salt & 5 & $10 / 30 / 97$ & 13497 & 60 & 26.2 & & S97T002217 \\
\hline SX-106 & 223 & Salt & 8 & $10 / 30 / 97$ & 11863 & 125 & 84.9 & & S97T002222 \\
\hline SX-106 & 223 & Salt & 8 & $10 / 30 / 97$ & 12908 & 250 & 98 & & S97T002228 \\
\hline SX-106 & 223 & Salt & 8 & $10 / 31 / 97$ & 13503 & 60 & 110.3 & & S97T002230 \\
\hline & & & & & & Total Weight & 548.5 & & \\
\hline SX-106 & 223 & Sludge & 10 & $11 / 12 / 97$ & 13622 & 60 & 122 & & S97T002261 \\
\hline SX-106 & 223 & Sludge & 7 & $10 / 21 / 97$ & 13505 & 60 & 93.8 & & S97T002221 \\
\hline SX-106 & 223 & Sludge & 7 & $10 / 30 / 97$ & 12895 & 250 & 105.8 & & S97T002219 \\
\hline
\end{tabular}


Vials in 11A1 and 11A2

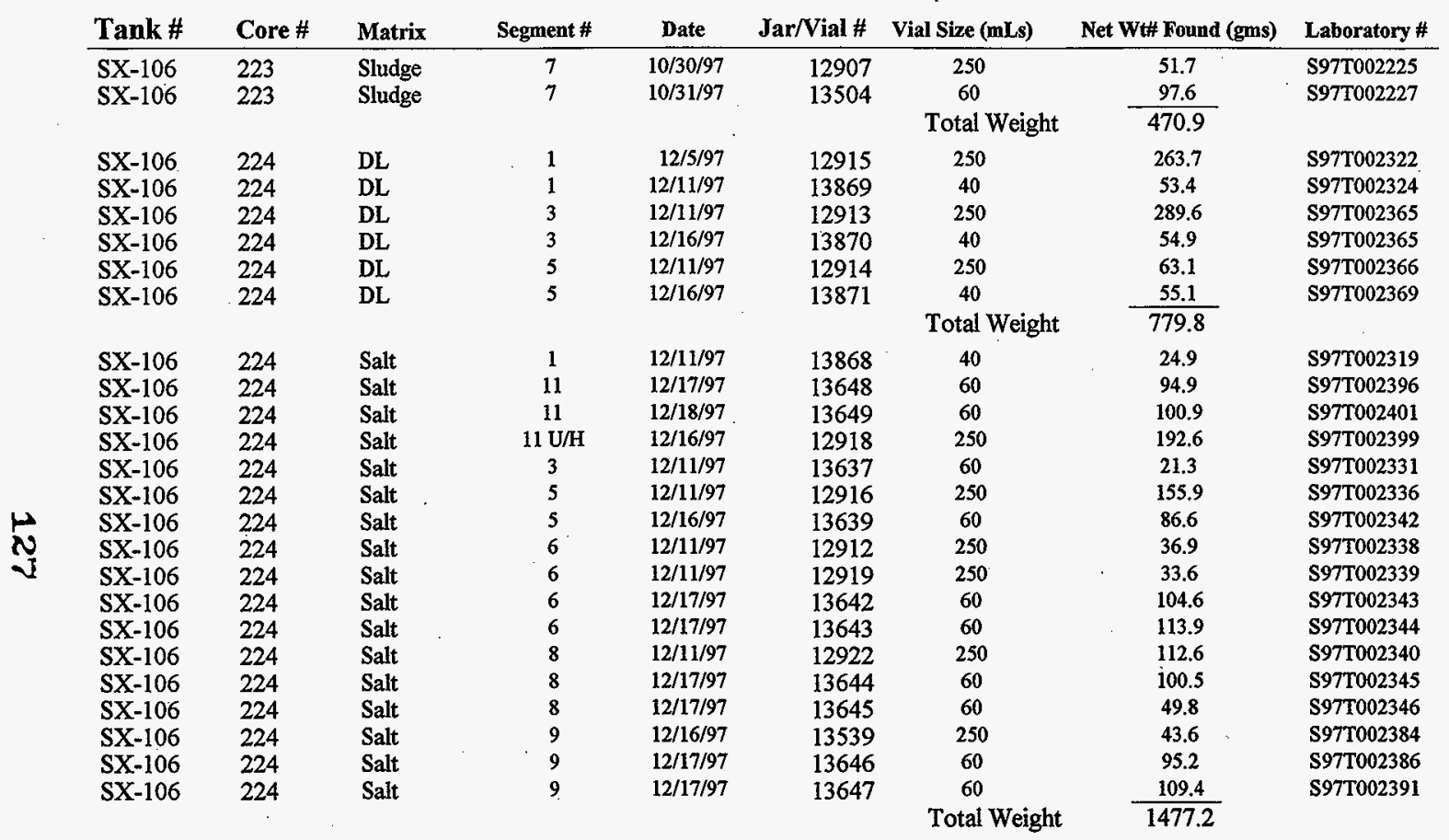

\begin{tabular}{lllccccrr} 
Tank \# & Core \# & Matrix & Segment \# & Date & Jar/Vial \# & Vial Size (mLs) & Net Wt\# Found (gms) & Laboratory \# \\
\hline SX-108 & R16 & Sludge & Aug-43 & $10 / 2 / 95$ & 7978 & 40 & 55.4 & S95T002575 \\
SX-108 & R16 & Sludge & Aug-43 & $10 / 2 / 95$ & 8115 & 20 & 3.5 & S95T002577
\end{tabular}


Vials in 11A1 and 11A2

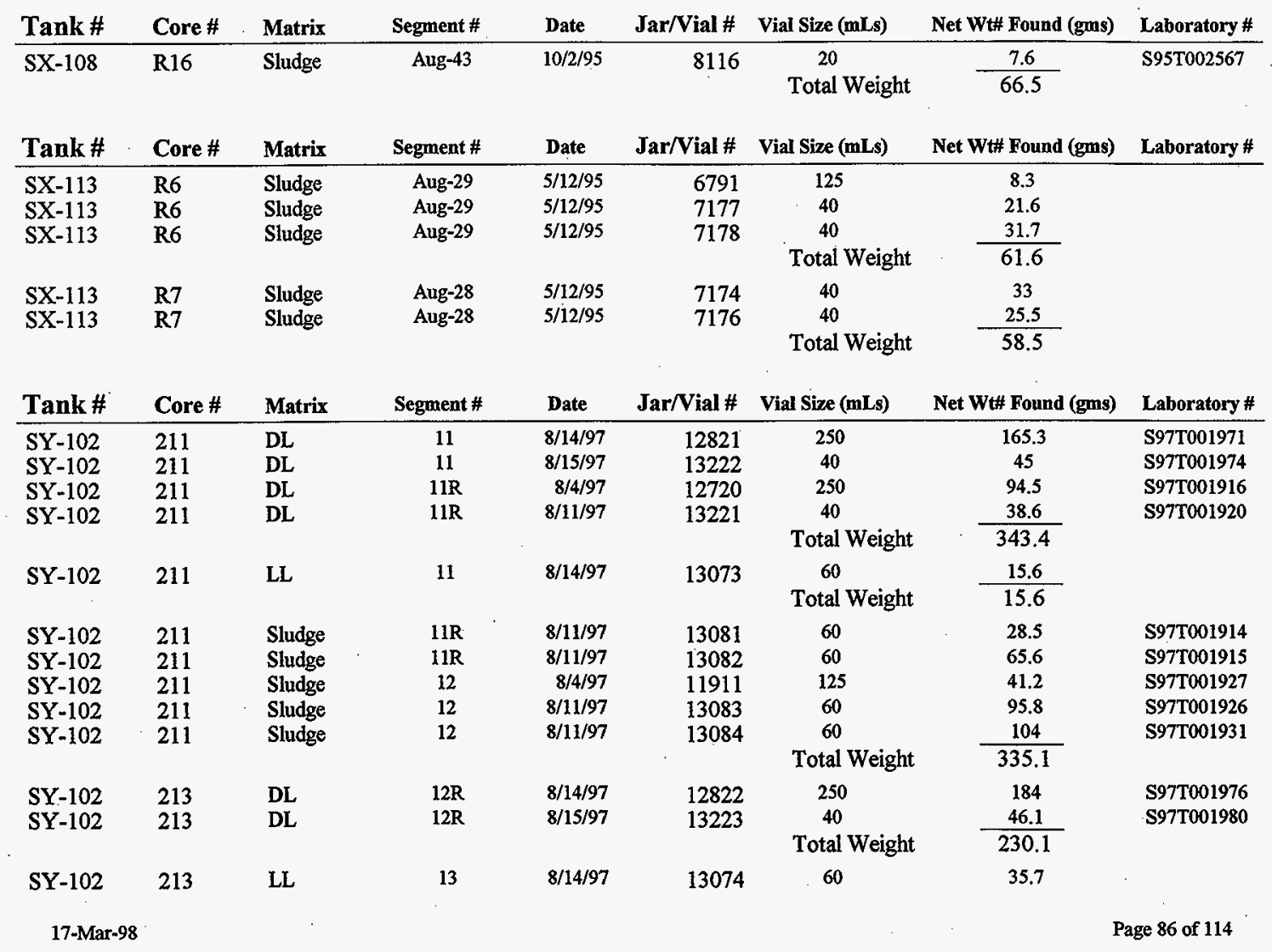


Vials in 11A1 and 11A2

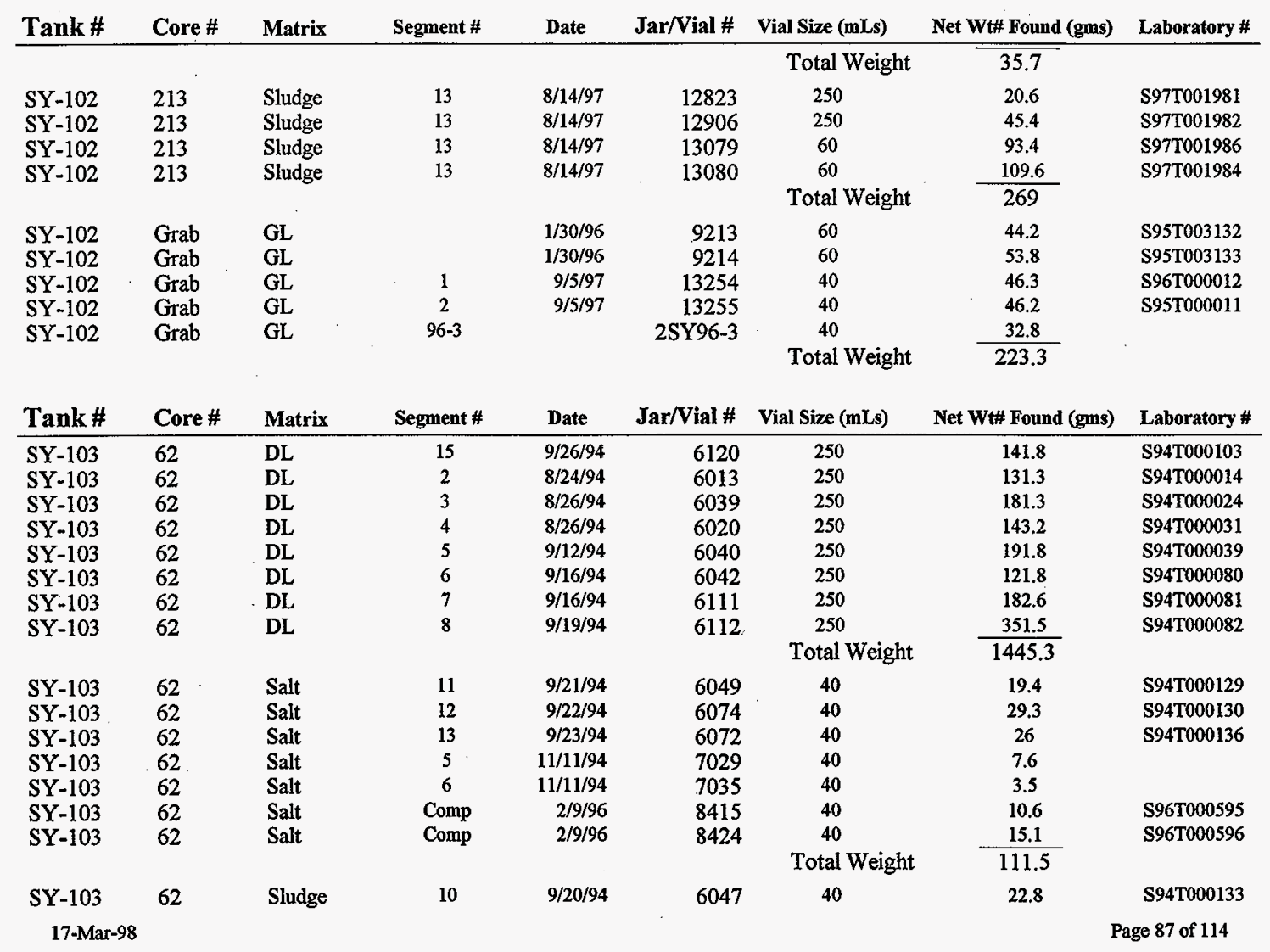


Vials in 11A1 and 11A2

\begin{tabular}{|c|c|c|c|c|c|c|c|c|}
\hline Tank \# & Core\# & Matrix & Segment \# & Date & Jar/Vial \# & Vial Size (mLs) & Net Wt\# Found (gms) & Laboratory \# \\
\hline SY-103 & 62 & Sludge & 10 & $9 / 20 / 94$ & 6050 & 40 & 23.6 & S94T000128 \\
\hline SY-103 & 62 & Sludge & 11 & $9 / 21 / 94$ & 6048 & 40 & 20.4 & S94T000134 \\
\hline SY-103 & 62 & Sludge & 12 & $9 / 22 / 94$ & 6073 & 40 & 17.9 & S94T000135 \\
\hline SY -103 & 62 & Sludge & 13 & $9 / 23 / 94$ & 6075 & 40 & 24.6 & S94T000131 \\
\hline SY -103 & 62 & Sludge & 14 & $9 / 23 / 94$ & 6076 & 40 & 24.3 & S94T000132 \\
\hline SY-103 & 62 & Sludge & 14 & $9 / 23 / 94$ & 6077 & 40 & 18.7 & S94T000137 \\
\hline SY -103 & 62 & Sludge & 15 & $9 / 26 / 94$ & 6169 & 40 & 4.6 & S94T000102 \\
\hline SY -103 & 62 & Sludge & 9 & $9 / 8 / 97$ & 12824 & 250 & 310.9 & S94T000077 \\
\hline \multirow[t]{2}{*}{ SY-103 } & 62 & Sludge & Comp & $9 / 26 / 97$ & 13456 & 60 & 25.9 & S94T000271 \\
\hline & & & & & & Total Weight & 493.7 & \\
\hline
\end{tabular}

\begin{tabular}{|c|c|c|c|c|c|c|c|}
\hline Tank \# & Core \# & Matrix & Segment \# & Date & Jar/Vial \# & Vial Size (mLs) & Net Wt\# Found \\
\hline T-104 & 45 & Sludge & 5 & $1 / 9 / 95$ & K130 & 40 & 21.7 \\
\hline T-104 & 45 & Sludge & 7 & $1 / 9 / 95$ & K132 & 40 & 18.2 \\
\hline T-104 & 45 & Sludge & 8 & $1 / 9 / 95$ & K133 & 40 & 10.6 \\
\hline $\mathrm{T}-104$ & 45 & Sludge & 9 & $1 / 9 / 95$ & K134 & 40 & 15.2 \\
\hline \multirow[t]{2}{*}{ T-104 } & 45 & Sludge & Comp & $9 / 3 / 97$ & 13518 & 60 & 68 \\
\hline & & & & & & Total Weight & 133.7 \\
\hline
\end{tabular}

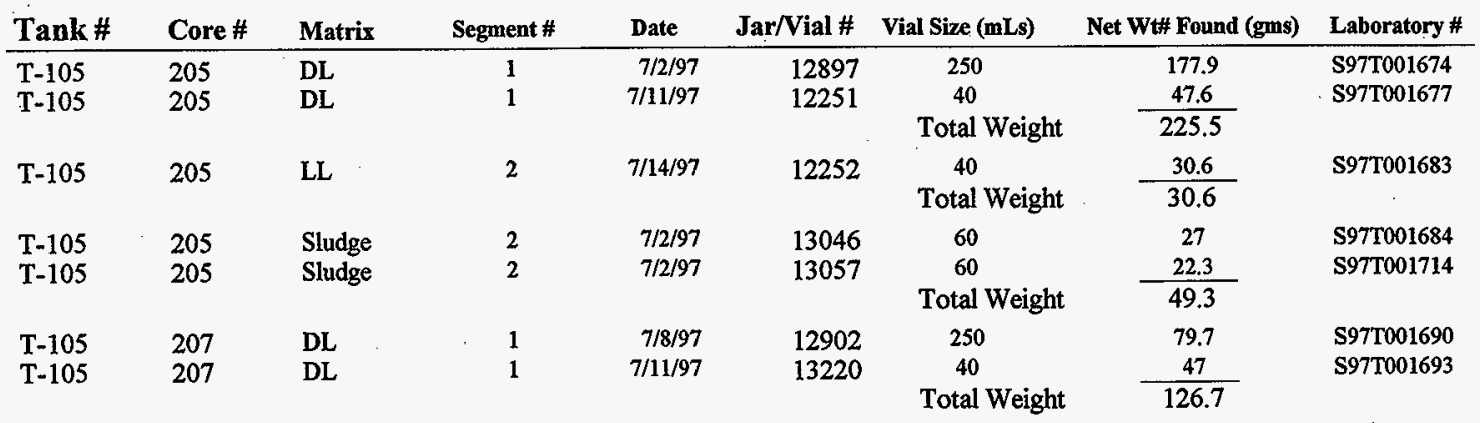




\section{Vials in 11A1 and 11A2}

\begin{tabular}{lllcccccc} 
Tank \# & Core \# & Matrix & Segment \# & Date & Jar/Vial \# & Vial Size (mLs) & Net Wt\# Found (gms) & Laboratory \# \\
\hline T-105 & 207 & Sludge & 1 & $7 / 11 / 97$ & 13054 & 60 & 42.8 & S97T001701 \\
T-105 & 207 & Sludge & 2 & $7 / 8 / 97$ & 12599 & 250 & 33.1 & S97T001708 \\
T-105 & 207 & Sludge & 2 & $7 / 8 / 97$ & 12903 & 250 & S97T001702 \\
T-105 & 207 & Sludge & 2 & $7 / 14 / 97$ & 13055 & 60 & 92.8 & S97T001707 \\
T-105 & 207 & Sludge & 2 & $7 / 14 / 97$ & 13056 & 60 & Total Weight & S97T001710 \\
& & & & & & & &
\end{tabular}

\begin{tabular}{|c|c|c|c|c|c|c|c|c|}
\hline Tank \# & Core\# & Matrix & Segment \# & Date & Jar/Vial \# & Vial Size (mLs) & Net Wt\# Found (gms) & Laboratory \# \\
\hline $\mathrm{T}-106$ & & Sludge & Aug-39 & $8 / 1 / 95$ & 7453 & $\begin{array}{c}40 \\
\text { Total Weight }\end{array}$ & $\begin{array}{r}12.9 \\
12.9\end{array}$ & S95T001462 \\
\hline
\end{tabular}

$\begin{array}{cccccccccc}\text { T-106 } & \text { R5 } & \text { Sludge } & \text { Aug-38 } & 8 / 1 / 95 & 7430 & 40 & 29 & \text { S95T001345 }\end{array}$

Tank \# Core\# Matrix Segment\# Date Jar/Vial \# Vial Size (mLs) ; Net Wt\# Found (gms) Laboratory \#

\begin{tabular}{|c|c|c|c|c|c|c|}
\hline$T-107$ & Sludge & Comp & $9 / 22 / 97$ & 13276 & 40 & 9.2 \\
\hline & & & & & Total Weight & 9.2 \\
\hline
\end{tabular}

\begin{tabular}{lllcccccc} 
Tank \# & Core \# & Matrix & Segment \# & Date & Jar/Vial \# & Vial Size (mLs) & Net Wt\# Found (gms) & Laboratory \# \\
\hline T-108 & R2 & Salt & Aug-37 & $7 / 27 / 95$ & 7429 & 40 & Total Weight & $\frac{18.6}{18.6}$ \\
& & & & & & S95T001334
\end{tabular}

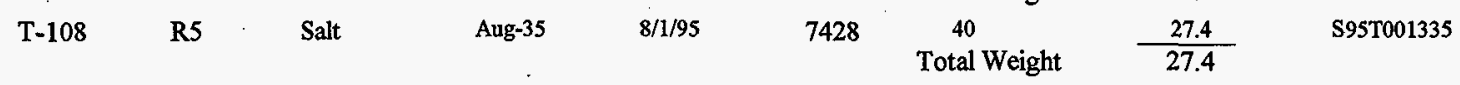

\begin{tabular}{|c|c|c|c|c|c|c|c|c|}
\hline Tank \# & Core \# & Matrix & Segment \# & Date & Jar/Vial \# & Vial Size (mLs) & Net Wt\# Found (gms) & Laboratory \# \\
\hline$T-109$ & & Salt & Aug-40 & $9 / 7 / 95$ & 7659 & 40 & 18.8 & S95T001604 \\
\hline $\mathrm{T}-109$ & & Salt & Aug-40 & $9 / 26 / 97$ & 13452 & 60 & 19.9 & S95T001599 \\
\hline $\mathrm{T}-109$ & & Salt & Aug-41 & $8 / 25 / 95$ & 7467 & $\begin{array}{c}40 \\
\text { Total Weight }\end{array}$ & $\frac{34.7}{73.4}$ & S95T001625 \\
\hline T-109 & & Sludge & Aug-41 & $9 / 7 / 95$ & 7660 & 40 & 21 & \\
\hline
\end{tabular}


Vials in 11A1 and 11A2

\begin{tabular}{|c|c|c|c|c|c|c|c|c|}
\hline Tank\# & Core \# & Matrix & Segment \# & Date & Jar/Vial \# & Vial Size (mLs) & Net Wt\# Found (gms) & Laboratory \# \\
\hline $\mathrm{T}-109$ & & Sludge & Aug-41 & $9 / 26 / 97$ & 11868 & $\begin{array}{c}125 \\
\text { Total Weight }\end{array}$ & $\frac{79.7}{100.7}$ & S95T001793 \\
\hline $\mathrm{T}-109$ & 103 & Salt & Aug-41 & $8 / 25 / 95$ & 7462 & $\begin{array}{l}40 \\
\text { Total Weight }\end{array}$ & $\begin{array}{r}39.6 \\
39.6\end{array}$ & S95T001622 \\
\hline
\end{tabular}

\begin{tabular}{|c|c|c|c|c|c|c|c|c|}
\hline Tank\# & Core \# & Matrix & Segment \# & Date & Jar/Vial \# & Vial Size (mLs) & Net Wt\# Found (gms) & Laboratory \# \\
\hline$T-110$ & 180 & Sludge & 1 & $2 / 18 / 97$ & 12159 & 250 & 46.1 & S97T000206 \\
\hline $\mathrm{T}-110$ & 180 & Sludge & 1 & $2 / 18 / 97$ & 121.60 & 250 & 63.5 & S97T000216 \\
\hline $\mathrm{T}-110$ & 180 & Sludge & 1 & $2 / 26 / 97$ & 12096 & 60 & 53.7 & S97T000223 \\
\hline T-110 & 180 & Sludge & 1 & $2 / 26 / 97$ & 12097 & 60 & 64.8 & S97T000215 \\
\hline $\mathrm{T}-110$ & 180 & Sludge & 2 & $2 / 26 / 97$ & 12169 & 250 & 38.5 & S97T000249 \\
\hline $\mathrm{T}-110$ & 180 & Sludge & 2 & $2 / 26 / 97$ & 12170 & 250 & 60.9 & S97T000250 \\
\hline $\mathrm{T}-110$ & 180 & Sludge & 2 & $3 / 5 / 97$ & 11723 & 60 & 64.2 & S97T000255 \\
\hline $\mathrm{T}-110$ & 180 & Sludge & 2 & $3 / 5 / 97$ & 12617 & 60 & 66.8 & S97T000256. \\
\hline $\mathrm{T}-110$ & 180 & Sludge & 3 & $2 / 18 / 97$ & 12161 & 250 & 61.9 & S97T000208 \\
\hline $\mathrm{T}-110$ & 180 & Sludge & 3 & $2 / 18 / 97$ & 12162 & 250 & 67.3 & S97T000209 \\
\hline $\mathrm{T}-110$ & 180 & Sludge & 3 & $2 / 27 / 97^{\text {? }}$ & 11722 & 60 & 65.4 & S97T000225 \\
\hline $\mathrm{T}-110$ & 180 & Sludge & 3 & $2 / 27 / 97$ & 12100 & 60 & 59.1 & S97T000224 \\
\hline $\mathrm{T}-110$ & 180 & Sludge & 4 & $2 / 18 / 97$ & 12163 & 250 & 220 & S97T000210 \\
\hline $\mathrm{T}-110$ & 180 & Sludge & 4 & $2 / 18 / 97$ & 12164 & 250 & 38.8 & S97T000211 \\
\hline $\mathrm{T}-110$ & 180 & Sludge & 4 & $2 / 27 / 97$ & 11724 & 60 & 62.5 & S97T000227 \\
\hline $\mathrm{T}-110$ & 180 & Sludge & 4 & $2 / 27 / 97$ & 11725 & 60 & 62.3 & S97T000226 \\
\hline $\mathrm{T}-110$ & 180 & Sludge & 6 & $2 / 18 / 97$ & 12165 & 250 & 56.1 & S97T000212 \\
\hline $\mathrm{T}-110$ & 180 & Sludge & 6 & $2 / 18 / 97$ & 12166 & 250 & 91.4 & S97T000213 \\
\hline $\mathrm{T}-110$ & 180 & Sludge & 6 & $2 / 26 / 97$ & 11726 & 60 & 50.7 & S97T000229 \\
\hline T-110 & 180 & Sludge & 6 & $2 / 26 / 97$ & 11727 & 60 & 51.6 & S97T000228 \\
\hline $\mathrm{T}-110$ & 180 & Sludge & 7 & $2 / 26 / 97$ & 12168 & 250 & 72.8 & S97T000252 \\
\hline $\mathrm{T}-110$ & 180 & Sludge & 7 & $3 / 6 / 97$ & 12618 & 60 & 58.6 & S97T000257 \\
\hline $\mathrm{T}-110$ & 180 & Sludge & 7 & $3 / 6 / 97$ & 12619 & 60 & 65.2 & S97T000258 \\
\hline $\mathrm{T}-110$ & 180 & Sludge & 8 & $2 / 27 / 97$ & 12172 & 250 & 108.7 & S97T000254 \\
\hline T-110 & 180 & Sludge & 8 & $3 / 6 / 97$ & 12620 & 60 & 57.7 & S97T000259 \\
\hline T-110 & 180 & Sludge & 8 & $3 / 6 / 97$ & 12621 & 60 & 69.7 & S97T000260 \\
\hline
\end{tabular}


Vials in 11A1 and 11A2

\begin{tabular}{|c|c|c|c|c|c|c|c|c|}
\hline Tank \# & Core \# & Matrix & Segment \# & Date & Jar/Vial \# & Vial Size (mLs) & Net Wt\# Found (gms) & Laboratory \# \\
\hline & & & & & & Total Weight & $\overline{1778.3}$ & \\
\hline $\mathrm{T}-110$ & 181 & FB & Fblk & $2 / 7 / 97$ & 12151 & 250 & 166.4 & S97T000116 \\
\hline $\mathrm{T}-110$ & 181 & FB & Fblk & $2 / 11 / 97$ & 12211 & 40 & 38.2 & S97T000122 \\
\hline & & & & & & Total Weight & 204.6 & \\
\hline $\mathrm{T}-110$ & 181 & Sludge & 1 & $2 / 7 / 97$ & 11930 & 250 & 60.5 & S97T000127 \\
\hline $\mathrm{T}-110$ & 181 & Sludge & 1 & $2 / 11 / 97$ & 12079 & 60 & 72.5 & S97T000126 \\
\hline $\mathrm{T}-110$ & 181 & Sludge & 1 & $2 / 11 / 97$ & 12081 & 60 & 67.8 & \$97T000134 \\
\hline $\mathrm{T}-110$ & 181 & Sludge & 2 & $2 / 6 / 97$ & 11929 & 250 & 18.6 & S97T000128 \\
\hline $\mathrm{T}-110$ & 181 & Sludge & 2 & $2 / 6 / 97$ & 11939 & 250 & 23 & S97T000129 \\
\hline $\mathrm{T}-110$ & 181 & Sludge & 2 & $2 / 11 / 97$ & 12077 & 60 & 48.3 & S97T000136 \\
\hline T-110 & 181 & Sludge & 2 & $2 / 11 / 97$ & 12083 & 60 & 72.1 & S97T000135 \\
\hline $\mathrm{T}-110$ & 181 & Sludge & 3 & $2 / 7 / 97$ & 12149 & 250 & 209 & S97T000130 \\
\hline $\mathrm{T}-110$ & 181 & Sludge & 3 & $2 / 7 / 97$ & 12150 & 250 & 60.4 & S97T000131 \\
\hline $\mathrm{T}-110$ & 181 & Sludge & 3 & $2 / 13 / 97$ & 12089 & 60 & 62.4 & S97T000137 \\
\hline $\mathrm{T}-110$ & 181 & Sludge & 3 & $2 / 13 / 97$ & 12090 & 60 & 71.3 & S97T000138 \\
\hline $\mathrm{T}-110$ & 181 & Sludge & 4 & $2 / 6 / 97$ & 11941 & 250 & 60.5 & S97T000133 \\
\hline $\mathrm{T}-110$ & 181 & Sludge & 4 & $2 / 13 / 97$ & 12091 & 60 & 43.8 & S97T000139 \\
\hline $\mathrm{T}-110$ & 181 & Sludge & 4 & $2 / 13 / 97$ & 12092 & 60 & 30.6 & S97T000140 \\
\hline$T-110$ & 181 & Sludge & 5 & $2 / 13 / 97$ & 12153 & 250 & 43 & \$97T000165 \\
\hline $\mathrm{T}-110$ & 181 & Sludge & 5 & $2 / 13 / 97$ & 12154 & 250 & 70.9 & S97T000166 \\
\hline $\mathrm{T}-110$ & 181 & Sludge & 5 & $3 / 7 / 97$ & 12105 & 60 & 57.6 & S97T000171 \\
\hline $\mathrm{T}-110$ & 181 & Sludge & 5 & $3 / 7 / 97$ & 12106 & 60 & 51.8 & S97T000167 \\
\hline $\mathrm{T}-110$ & 181 & Sludge & 6 & $2 / 13 / 97$ & 12155 & 250 & 44.8 & S97T000172 \\
\hline $\mathrm{T}-110$ & 181 & Sludge & 6 & $2 / 13 / 97$ & 12156 & 250 & 78.1 & S97T000173 \\
\hline $\mathrm{T}-110$ & 181 & Sludge & 6 & $3 / 7 / 97$ & 11815 & 60 & 72.7 & S97T000177 \\
\hline $\mathrm{T}-110$ & 181 & Sludge & 6 & $3 / 7 / 97$ & 11816 & 60 & 48.3 & S97T000176 \\
\hline $\mathrm{T}-110$ & 181 & Sludge & 7 & $2 / 11 / 97$ & 11933 & 250 & 61.9 & S97T000157 \\
\hline $\mathrm{T}-110$ & 181 & Sludge & 7 & $2 / 13 / 97$ & 12088 & 60 & 66.7 & S97T000160 \\
\hline $\mathrm{T}-110$ & 181 & Sludge & 7 & $2 / 13 / 97$ & 12093 & 60 & 73.1 & S97T000161 \\
\hline $\mathrm{T}-110$ & 181 & Sludge & 7 & $2 / 13 / 97$ & 12152 & 250 & 23.5 & S97T000156 \\
\hline $\mathrm{T}-110$ & 181 & Sludge & 8 & $2 / 18 / 97$ & 12157 & 250 & 79.9 & S97T000190 \\
\hline $\mathrm{T}-110$ & 181 & Sludge & 8 & $2 / 18 / 97$ & 12158 & 250 & 84.5 & S97T000191 \\
\hline $\mathrm{T}-110$ & 181 & Sludge & 8 & $2 / 27 / 97$ & 12094 & 60 & 57.8 & S97T000194 \\
\hline
\end{tabular}


Vials in 11A1 and 11A2

\begin{tabular}{lllcccccc} 
Tank\# & Core \# & Matrix & Segment \# & Date & Jar/Vial \# & Vial Size (mLs) & Net Wt\# Found (gms) & Lahoratory \# \\
\hline T-110 & 181 & Sludge & 8 & $2 / 27 / 97$ & 12095 & 60 & $\frac{57.4}{1872.8}$ & T97T000195
\end{tabular}

\begin{tabular}{|c|c|c|c|c|c|c|c|c|}
\hline Tank \# & Core\# & Matrix & Segment \# & Date & Jar/Vial \# & Vial Size (mLs) & Net Wt\# Found (gms) & Laboratory \# \\
\hline $\mathrm{T}-112$ & 185 & DL & 1 & $3 / 11 / 97$. & 12193 & 250 & 253.6 & \\
\hline $\mathrm{T}-112$ & 185 & DL & 1 & $3 / 12 / 97$ & 12215 & 40 & 46.1 & S97T000375 \\
\hline $\mathrm{T}-112$ & 185 & DL & 2 & $3 / 11 / 97$ & 11872 & 125 & 19.7 & S97T000366 \\
\hline \multirow[t]{2}{*}{$\mathrm{T}-112$} & 185 & $\overline{\mathrm{DL}}$ & 2 & $3 / 12 / 97$ & 12221 & 40 & 44.3 & S97T000378 \\
\hline & & & & & & Total Weight & 363.7 & \\
\hline $\mathrm{T}-112$ & 185 & FB & Folk & $3 / 12 / 97$ & 12180 & 250 & 159.2 & S97T000367 \\
\hline \multirow[t]{2}{*}{$\mathrm{T}-112$} & 185 & FB & Fblk & $3 / 12 / 97$ & 12222 & 40 & 41 & \$97T000379 \\
\hline & & & & & & Total Weight & 200.2 & \\
\hline $\mathrm{T}-112$ & 185 & Sludge & 2 & $3 / 11 / 97$ & 11873. & 125 & 12.3 & S97T000372 \\
\hline $\mathrm{T}-112$ & 185 & Sludge & 2 & $3 / 12 / 97$ & 12114 & 60 & 54.1 & S97T000373 \\
\hline \multirow[t]{2}{*}{$\mathrm{T}-112$} & 185 & Sludge & 2 & $3 / 12 / 97$ & 12119 & 60 & 74.4 & S97T000372 \\
\hline & & & & & & Total Weight & 140.8 & \\
\hline $\mathrm{T}-112$ & 186 & Sludge & 2 & $3 / 31 / 97$ & 12182 & 250 & 26.2 & S97T000433 \\
\hline $\mathrm{T}-112$ & 186 & Sludge & 2 & $3 / 31 / 97$ & 12183 & 250 & 38 & S97T 000434 \\
\hline $\mathrm{T}-112$ & 186 & Sludge & 2 & $4 / 4 / 97$ & 12304 & 60 & 58 & S97Tr000449 \\
\hline \multirow[t]{2}{*}{$\mathrm{T}-112$} & 186 & Sludge & 2 & $4 / 4 / 97$ & 12625 & 60 & 77.2 & S97T000450 \\
\hline & & & & & & Total Weight & 199.4 & \\
\hline
\end{tabular}

\begin{tabular}{|c|c|c|c|c|c|c|c|c|}
\hline Tank \# & Core \# & Matrix & Segment \# & Date & Jar/Vial \# & Vial Size (mLs) & Net WtH Found (gms) & Laboratory \# \\
\hline $\mathrm{T}-201$ & 192 & DL & 1 & $5 / 6 / 97$ & 12278 & 250 & 163.5 & S97T000825 \\
\hline T-201 & 192 & DL & 1 & $5 / 20 / 97$ & 12232 & 40 & 42.7 & S97T000838 \\
\hline T-201 & 192 & DL & 2 & $5 / 20 / 97$ & 12235 & 40 & 42.1 & \$97T00839 \\
\hline T-201 & 192 & DL & 3 & $5 / 20 / 97$ & 12236 & 40 & 45.6 & S97T000840 \\
\hline T-201 & 192 & $\mathrm{DL}$ & 4 & $5 / 20 / 97$ & 12237 & 40 & 43.2 & S97T000841 \\
\hline $\mathrm{T}-201$ & 192 & $\overline{\mathrm{DL}}$ & 5 & $5 / 20 / 97$ & 12238 & 40 & 40.7 & S97T000842 \\
\hline \multirow[t]{2}{*}{$\mathrm{T}-201$} & 192 & DL & Comp & $6 / 24 / 97$ & 12740 & 60 & 36.5 & S97T001254 \\
\hline & & & & & & Total Weight & 414.3 & \\
\hline
\end{tabular}


Vials in 11A1 and 11A2

\begin{tabular}{|c|c|c|c|c|c|c|c|c|}
\hline Tank\# & Core \# & Matrix & Segment \# & Date & Jar/Vial \# & Vial Size (mLs) & Net Wt\# Found (gms) & Laboratory\# \\
\hline $\begin{array}{l}T-201 \\
\text { T-201 }\end{array}$ & $\begin{array}{l}192 \\
192\end{array}$ & $\begin{array}{l}\text { FB } \\
\text { FB }\end{array}$ & $\begin{array}{l}\text { Fblk } \\
\text { Fblk }\end{array}$ & $\begin{array}{r}5 / 6 / 97 \\
5 / 20 / 97\end{array}$ & $\begin{array}{l}12273 \\
12223\end{array}$ & $\begin{array}{c}250 \\
40 \\
\text { Total Weight }\end{array}$ & $\begin{array}{c}153.2 \\
42.2 \\
195.4\end{array}$ & $\begin{array}{l}\text { S97T000822 } \\
\text { S97T000824 }\end{array}$ \\
\hline $\mathrm{T}-201$ & 192 & Sludge & 6 & $5 / 8 / 97$ & 12603 & 250 & 45 & S97T000894 \\
\hline $\mathrm{T}-201$ & 192 & Sludge & 6 & $5 / 8 / 97$ & 12605 & 250 & 38.2 & S97T000897 \\
\hline $\mathrm{T}-201$ & 192 & Sludge & 6 & $5 / 20 / 97$ & 12336 & 60 & 72.6 & S97T000898 \\
\hline $\mathrm{T}-201$ & 192 & Sludge & 6 & $5 / 20 / 97$ & 12337 & 60 & 72.9 & S97T000904 \\
\hline $\mathrm{T}-201$ & 192 & Sludge & 7 & $5 / 8 / 97$ & 12608 & 250 & 58.5 & S97T000902 \\
\hline T-201 & 192 & Sludge & 7 & $5 / 8 / 97$ & 12615 & 250 & 28.8 & S97T000895 \\
\hline $\mathrm{T}-201$ & 192 & Sludge & 7 & $5 / 20 / 97$ & 12339 & 60 & 89.6 & S97T000906 \\
\hline $\mathrm{T}-201$ & 192 & Sludge & 7 & $5 / 21 / 97$ & 12338 & 60 & 65 & S97T000905 \\
\hline $\mathrm{T}-201$ & 192 & Sludge & 8 & $5 / 8 / 97$ & 12601 & 250 & 16.6 & S97T000896 \\
\hline T-201 & 192 & Sludge & 8 & $5 / 8 / 97$ & 12606 & 250 & 67.1 & S97T000903 \\
\hline $\mathrm{T}-201$ & 192 & Sludge & 8 & $5 / 21 / 97$ & 12340 & 60 & 78.4 & S97T000907 \\
\hline $\mathrm{T}-201$ & 192 & Sludge & 8 & $5 / 21 / 97$ & 12341 & 60 & 83.7 & S97T000908 \\
\hline $\mathrm{T}-201$ & 192 & Sludge & Comp & $6 / 23 / 97$ & 12665 & 125 & 9.2 & S97T001245 \\
\hline $\mathrm{T}-201$ & 192 & Sludge & Comp & $6 / 23 / 97$ & 12739 & 60 & 29.7 & S97T001247 \\
\hline & & & & & & Total Weight & 755.3 & \\
\hline
\end{tabular}

\begin{tabular}{|c|c|c|c|c|c|c|c|c|}
\hline Tank\# & Core \# & Matrix & Segment \# & Date & Jar/Vial \# & Vial Size (mLs) & Net Wt\# Found (gms) & Laboratory \# \\
\hline $\mathrm{T}-202$ & 191 & FB & Fblk & $5 / 1 / 97$ & 12274 & 250 & 120.3 & S97T000785 \\
\hline \multirow[t]{2}{*}{$\mathrm{T}-202$} & 191 & FB & Folk & $5 / 12 / 97$ & 12230 & 40 & 40.3 & S97T000801 \\
\hline & & & & & & & & \\
\hline $\mathrm{T}-202$ & 191 & Sludge & 1 & $5 / 1 / 97$ & 12276 & 250 & 10.7 & S97T000786 \\
\hline$T-202$ & 191 & Sludge & 1 & $5 / 12 / 97$ & 12331 & 60 & 78.9 & S97T000812 \\
\hline T-202 & 191 & Sludge & 1 & $6 / 11 / 97$ & 12332 & 60 & 65.9 & S97T000813 \\
\hline T-202 & 191 & Sludge & 2 & $5 / 2 / 97$ & 12359 & 125 & 32.8 & S97T000792 \\
\hline $\mathrm{T}-202$ & 191 & Sludge & 2 & $5 / 12 / 97$ & 12333 & 60 & 76.8 & S97T000814 \\
\hline T-202 & 191 & Sludge & 2 & $5 / 12 / 97$ & 12334 & 60 & 73.7 & S97T000815 \\
\hline $\mathrm{T}-202$ & 191 & Sludge & 3 & $5 / 1 / 97$ & 12275 & 250 & 9.6 & S97T000788 \\
\hline T-202 & 191 & Sludge & 3 & $5 / 12 / 97$ & 12635 & 60 & 82.9 & S97T000816 \\
\hline T-202 & 191 & Sludge & 3 & $5 / 12 / 97$ & 12636 & 60 & 8.3 & S97T000817 \\
\hline
\end{tabular}


Vials in 11A1 and 11A2

\begin{tabular}{|c|c|c|c|c|c|c|c|c|}
\hline Tank \# & Core \# & Matrix & Segment \# & Date & Jar/Vial \# & Vial Size (mLs) & Net Wt\# Found (gms) & Laboratory \# \\
\hline $\mathrm{T}-202$ & 191 & Sludge & 4 & $5 / 2 / 97$ & 12270 & 250 & 21.6 & S97T000789 \\
\hline $\mathrm{T}-202$ & 191 & Sludge & 4 & $5 / 2 / 97$ & 12277 & 250 & 10.9 & S97T000794 \\
\hline T-202 & 191 & Sludge & 4 & $5 / 13 / 97$ & 12637 & 60 & 76.5 & S97T000818 \\
\hline $\mathrm{T}-202$ & 191 & Sludge & 4 & $5 / 13 / 97$ & 12638 & 60 & 77.9 & S97T000819 \\
\hline $\mathrm{T}-202$ & 191 & Sludge & 5 & $5 / 2 / 97$ & 12271 & 250 & 105 & S97T000790 \\
\hline $\mathrm{T}-202$ & 191 & Sludge & 5 & $5 / 13 / 97$ & 12634 & 60 & 80.1 & \$97T000820 \\
\hline $\mathrm{T}-202$ & 191 & Sludge & 5 & $5 / 13 / 97$ & 12639 & 60 & 46.8 & S97T000821 \\
\hline \multirow[t]{2}{*}{$\mathrm{T}-202$} & 191 & Sludge & Comp & $6 / 19 / 97$ & 12743 & 60 & 56.6 & S97T001126 \\
\hline & & & & & & Total Weight & 915 & \\
\hline
\end{tabular}

\begin{tabular}{|c|c|c|c|c|c|c|c|c|c|}
\hline Tank \# & Core \# & Matrix & Segment \# & Date & Jar/Vial \# & Vial Size (mLs) & Net Wt\# Found & (gms) & Laboratory \# \\
\hline $\begin{array}{l}\text { T-203 } \\
\text { T-203 }\end{array}$ & $\begin{array}{l}190 \\
190\end{array}$ & $\begin{array}{l}\text { DL } \\
\text { DL }\end{array}$ & $\begin{array}{l}\mathrm{IR} \\
\mathrm{IR}\end{array}$ & $\begin{array}{r}4 / 28 / 97 \\
5 / 5 / 97\end{array}$ & $\begin{array}{l}12623 \\
12229\end{array}$ & $\begin{array}{c}60 \\
40 \\
\text { Total Weight }\end{array}$ & $\begin{array}{c}1.2 \\
18.7 \\
19.9\end{array}$ & & $\begin{array}{l}\text { S97T000730 } \\
\text { S97T000734 }\end{array}$ \\
\hline $\begin{array}{l}\text { T-203 } \\
\text { T-203 }\end{array}$ & $\begin{array}{l}190 \\
190\end{array}$ & $\begin{array}{l}\text { FB } \\
\text { FB }\end{array}$ & $\begin{array}{l}\text { Fblk } \\
\text { Folk }\end{array}$ & $\begin{array}{r}4 / 23 / 97 \\
5 / 8 / 97\end{array}$ & $\begin{array}{l}12550 \\
11955\end{array}$ & $\begin{array}{c}250 \\
40 \\
\text { Total Weight }\end{array}$ & $\begin{array}{l}57.7 \\
38.9 \\
96.6\end{array}$ & . & $\begin{array}{l}\text { S97T000729 } \\
\text { S97T000732 }\end{array}$ \\
\hline $\begin{array}{l}\mathrm{T}-203 \\
\mathrm{~T}-203\end{array}$ & $\begin{array}{l}190 \\
190\end{array}$ & $\begin{array}{l}\text { Sludge } \\
\text { Sludge }\end{array}$ & $\begin{array}{l}1 \\
1\end{array}$ & $\begin{array}{l}4 / 23 / 97 \\
4 / 23 / 97\end{array}$ & $\begin{array}{l}12207 \\
12208\end{array}$ & $\begin{array}{l}250 \\
250\end{array}$ & $\begin{array}{l}32 \\
56\end{array}$ & & $\begin{array}{l}\text { S97T000657 } \\
\text { S97T000656 }\end{array}$ \\
\hline $\mathrm{T}-203$ & 190 & Sludge & 1 & $4 / 30 / 97$ & 12316 & 60 & 83 & & S97T000672 \\
\hline $\mathrm{T}-203$ & 190 & Sludge & 1 & $4 / 30 / 97$ & 12317 & 60 & 75.7 & & S97T000673 \\
\hline T-203 & 190 & Sludge & $1 R$ & $4 / 28 / 97$ & 12265 & 250 & 21 & & S97T000699 \\
\hline $\mathrm{T}-203$ & 190 & Sludge & IR & $4 / 28 / 97$ & 12352 & $125^{\circ}$ & 7.2 & & S97T000693 \\
\hline T-203 & 190 & Sludge & IR & $5 / 6 / 97$ & 12325 & 60 & 70.1 & & S97T000717 \\
\hline $\mathrm{T}-203$ & 190 & Sludge & 1R & $5 / 6 / 97$ & 12326 & 60 & 62.2 & & S97T000723 \\
\hline $\mathrm{T}-203$ & 190 & Sludge & 2 & $4 / 23 / 97$ & 12255 & 250 & 47.4 & & S97T000661 \\
\hline $\mathrm{T}-203$ & 190 & Sludge & 2 & $4 / 23 / 97$ & 12548 & 250 & 24.7 & & S97T000658 \\
\hline T-203 & 190 & Sludge & 2 & $4 / 30 / 97$ & 12318 & 60 & 73.3 & & S97T000674 \\
\hline $\mathrm{T}-203$ & 190 & Sludge & 2 & $4 / 30 / 97$ & 12319 & 60 & 78.3 & . & S97T000677 \\
\hline $\mathrm{T}-203$ & 190 & Sludge & 3 & $4 / 28 / 97$ & 12257 & 250 & 19.5 & & S97T000694 \\
\hline $\mathrm{T}-203$ & 190 & Sludge & 3 & $5 / 7 / 97$ & 12628 & 60 & 57.4 & & S97T000724 \\
\hline $\mathrm{T}-203$ & 190 & Sludge & 3 & $5 / 7 / 97$ & 12629 & 60 & 75.7 & & S97T000718 \\
\hline
\end{tabular}


Vials in 11A1 and 11A2

\begin{tabular}{|c|c|c|c|c|c|c|c|c|}
\hline Tank\# & Core \# & Matrix & Segment \# & Date & Jar/Vial \# & Vial Size (mLs) & Net Wt\# Found (gms) & Laboratory \# \\
\hline$T-203$ & 190 & Sludge & 4 & $4 / 23 / 97$ & 12256 & 250 & 65.6 & S97T000659 \\
\hline$T-203$ & 190 & Sludge & 4 & $4 / 30 / 97$ & 12320 & 60 & 79.6 & S97T000675 \\
\hline $\mathrm{T}-203$ & 190 & Sludge & 4 & 4/30/97 & 12321 & 60 & 77.2 & S97T000678 \\
\hline $\mathrm{T}-203$ & 190 & Sludge & 5 & $4 / 23 / 97$ & 12269 & 250 & 36.5 & S97T000663 \\
\hline $\mathrm{T}-203$ & 190 & Sludge & 5 & $5 / 7 / 97$ & 12322 & 60 & 80.8 & S97T000676 \\
\hline $\mathrm{T}-203$ & 190 & Sludge & 5 & $5 / 7 / 97$ & 12323 & 60 & 70.8 & S97T000679 \\
\hline $\mathrm{T}-203$ & 190 & Sludge & 6 & $4 / 28 / 97$ & 12264 & 250 & 69.2 & S97T000695 \\
\hline $\mathrm{T}-203$ & 190 & Sludge & 6 & $5 / 7 / 97$ & 11889 & 125 & 32.9 & S97T000701 \\
\hline $\mathrm{T}-203$ & 190 & Sludge & 6 & $5 / 7 / 97$ & 12630 & 60 & 64.7 & S97T000719 \\
\hline$T-203$ & 190 & Sludge & 6 & $5 / 7 / 97$ & 12631 & 60 & 63.8 & S97T000725 \\
\hline T-203 & 190 & Sludge & 7 & $4 / 29 / 97$ & 12260 & 250 & 75.2 & S97T000696 \\
\hline $\mathrm{T}-203$ & 190 & Sludge & 7 & $4 / 29 / 97$ & 12261 & 250 & 21.3 & S97T000702 \\
\hline$T-203$ & 190 & Sludge & 7 & $5 / 8 / 97$ & 12327 & 60 & 72 & S97T000720 \\
\hline T-203 & 190 & Sludge & 7 & $5 / 8 / 97$ & 12330 & 60 & 72.8 & S97T000726 \\
\hline$T-203$ & 190 & Sludge & 8 & $4 / 29 / 97$ & 12259 & 250 & 50.3 & S97T000703 \\
\hline $\mathrm{T}-203$ & 190 & Sludge & 8 & $4 / 29 / 97$ & 12262 & 250 & 53.9 & S97T000697 \\
\hline T-203 & 190 & Sludge & 8 & $5 / 9 / 97$ & 12632 & 60 & 78.6 & S97T000727 \\
\hline T-203 & 190 & Sludge & 8 & $5 / 9 / 97$ & 12633 & 60 & 83.4 & S97T000721 \\
\hline T-203 & 190 & Sludge & 9 & $4 / 29 / 97$ & 12258 & 250 & 54.2 & S97T000698 \\
\hline$T-203$ & 190 & Sludge & 9 & $4 / 30 / 97$ & 12263 & 250 & 28.6 & S97T000704 \\
\hline T-203 & 190 & Sludge & 9 & $5 / 8 / 97$ & 12328 & 60 & 71.6 & S97T000722 \\
\hline $\mathrm{T}-203$ & 190 & Sludge & 9 & $5 / 8 / 97$ & 12329 & 60 & 78.3 & S97T000728 \\
\hline T-203 & 190 & Sludge & Comp & $6 / 17 / 97$ & 12377 & 125 & 13.9 & S97T001012 \\
\hline \multirow[t]{2}{*}{$T-203$} & 190 & Sludge & Comp & $6 / 17 / 97$ & 12722 & 60 & 48.8 & S97T001014 \\
\hline & & & & & & Total Weight & 2227.5 & \\
\hline
\end{tabular}

\begin{tabular}{|c|c|c|c|c|c|c|c|c|}
\hline Tank \# & Core\# & Matrix & Segment \# & Date & Jar/Vial \# & Vial Size (mLs) & Net Wt\# Found (gms) & Laboratory \# \\
\hline $\mathrm{T}-204$ & 0 & Sludge & 4 & $4 / 22 / 97$ & 12121 & $\begin{array}{c}60 \\
\text { Total Weight }\end{array}$ & $\frac{79.1}{79.1}$ & S97T000581 \\
\hline $\begin{array}{l}\text { T-204 } \\
\text { T-204 }\end{array}$ & $\begin{array}{l}188 \\
188\end{array}$ & $\begin{array}{l}\text { FB } \\
\text { FB }\end{array}$ & $\begin{array}{l}\text { Fblk } \\
\text { Fblk }\end{array}$ & $\begin{array}{l}4 / 21 / 97 \\
4 / 24 / 97\end{array}$ & $\begin{array}{l}12546 \\
12228\end{array}$ & $\begin{array}{c}250 \\
40 \\
\text { Total Weight }\end{array}$ & $\begin{array}{c}189.2 \\
41.8 \\
231\end{array}$ & $\begin{array}{l}\text { S97T000645 } \\
\text { S97T000646 }\end{array}$ \\
\hline
\end{tabular}


Vials in 11A1 and 11A2

\begin{tabular}{|c|c|c|c|c|c|c|c|c|}
\hline Tank \# & Core \# & Matrix & Segment \# & Date & Jar/Vial \# & Vial Size (mLs) & Net Wt\# Found (gms) & Laboratory \# \\
\hline T-204 & 188 & Sludge & 1 & $4 / 9 / 97$ & 12189 & 250 & 47.4 & S97T000495 \\
\hline $\mathrm{T}-204$ & 188 & Sludge & 1 & 4/9/97 & 12197 & 250 & 23.1 & S97T000494 \\
\hline T-204 & 188 & Sludge & 1 & $4 / 21 / 97$ & 12145 & 60 & 81.6 & \$97T000497 \\
\hline T-204 & 188 & Sludge & 1 & $4 / 21 / 97$ & 12148 & 60 & 80.4 & \$97T000496 \\
\hline $\mathrm{T}-204$ & 188 & Sludge & 10 & $4 / 21 / 97$ & 12549 & 250 & 91 & S97T000632 \\
\hline$T-204$ & 188 & Sludge & 10 & $4 / 21 / 97$ & 12551 & 250 & 30.5 & S97T000614 \\
\hline $\mathrm{T}-204$ & 188 & Sludge & 10 & $4 / 29 / 97$ & 12314 & 60 & 55.2 & S97T000620 \\
\hline T-204 & 188 & Sludge & 10 & $4 / 29 / 97$ & 12315 & 60 & 73.1 & S97T000644 \\
\hline$T-204$ & 188 & Sludge & 2 & $4 / 22 / 97$ & 12118 & 60 & 41.2 & \$97T000577 \\
\hline $\mathrm{T}-204$ & 188 & Sludge & 3 & $4 / 16 / 97$ & 12535 & 250 & 76.1 & S97T000571 \\
\hline T-204 & 188 & Sludge & 3 & $4 / 22 / 97$ & 12120 & 60 & 84.1 & S97T000578 \\
\hline $\mathrm{T}-204$ & 188 & Sludge & 4 & $4 / 16 / 97$ & 12532 & 250 & 213.3 & S97T000572 \\
\hline $\mathrm{T}-204$ & 188 & Sludge & 4 & $4 / 16 / 97$ & 12537 & 250 & 63.8 & S97T000573 \\
\hline T-204 & 188 & Sludge & 4 & $4 / 22 / 97$ & 12122 & 60 & 75.1 & S97T000579 \\
\hline T-204 & 188 & Sludge & 5 & $4 / 18 / 97$ & 12533 & 250 & 67.3 & S97T000627 \\
\hline $\mathrm{T}-204$ & 188 & Sludge & 5 & $4 / 18 / 97$ & 12536 & 250 & 52.1 & S97T000609 \\
\hline T-204 & 188 & Sludge & 5 & $4 / 24 / 97$ & 12123 & 60 & 78.4 & S97T000615 \\
\hline T-204 & 188 & Sludge & 5 & $4 / 24 / 97$ & 12124 & 60 & 76.2 & S97T000639 \\
\hline T-204 & 188 & Sludge & 6 & $4 / 18 / 97$ & 12538 & 250 & 46.7 & S97T000610 \\
\hline $\mathrm{T}-204$ & 188 & Sludge. & 6 & $4 / 18 / 97$ & 12539 & 250 & 70.1 & S97T000628 \\
\hline T-204 & 188 & Sludge & 6 & $4 / 24 / 97$ & 12306 & 60 & 70.3 & S97T000616 \\
\hline $\mathrm{T}-204$ & 188 & Sludge & 6 & $4 / 24 / 97$ & 12307 & 60 & 84.9 & S97T000640 \\
\hline$T-204$ & 188 & Sludge & 7 & $4 / 18 / 97$ & 12540 & 250 & 47.6 & S97T000611 \\
\hline T-204 & 188 & Sludge & 7 & $4 / 18 / 97$ & 12544 & 250 & 73.9 & S97T000629 \\
\hline T-204 & 188 & Sludge & 7 & $4 / 28 / 97$ & 12308 & 60 & 76.7 & S97T000617 \\
\hline T-204 & 188 & Sludge & 7 & $4 / 28 / 97$ & 12309 & 60 & 74.3 & S97T000641 \\
\hline$T-204$ & 188 & Sludge & 8 & $4 / 18 / 97$ & 12542 & 250 & 48.8 & S97T000612 \\
\hline T-204 & 188 & Sludge & 8 & $4 / 18 / 97$ & 12543 & 250 & 48.4 & S97T000630 \\
\hline T-204 & 188 & Sludge & 8 & $4 / 29 / 97$ & 12310 & 60 & 78.2 & S $97 \mathrm{~T} 000642$ \\
\hline T-204 & 188 & Sludge & 8 & $4 / 29 / 97$ & 12311 & 60 & 78.7 & S97T000618 \\
\hline T-204 & 188 & Sludge & 9 & $4 / 21 / 97$ & 12541 & 250 & 45.1 & \$97T000613 \\
\hline $\mathrm{T}-204$ & 188 & Sludge & 9 & $4 / 21 / 97$ & 12545 & 250 & 76 & S97T000631 \\
\hline T-204 & 188 & Sludge & 9 & $4 / 29 / 97$ & 12312 & 60 & 54.3 & S97T000619 \\
\hline$T-204$ & 188 & Sludge & 9 & $4 / 29 / 97$ & 12313 & 60 & 58.9 & S $97 T 000643$ \\
\hline
\end{tabular}




\section{Vials in 11A1 and 11A2}

\begin{tabular}{|c|c|c|c|c|c|c|c|c|}
\hline Tank \# & Core \# & Matrix & Segment \# & Date & Jar/Vial \# & Vial Size (mLs) & Net Wt\# Found (gms) & Laboratory \# \\
\hline T-204 & 188 & Sludge & Comp & $6 / 12 / 97$ & 11835 & 125 & 28.1 & S97T001191 \\
\hline T-204 & 188 & Sludge & Comp & $6 / 12 / 97$ & 12693 & 60 & 42.5 & S97T001201 \\
\hline
\end{tabular}

\begin{tabular}{lllcccccc} 
Tank \# & Core \# & Matrix & Segment \# & Date & Jar/Vial \# & Vial Size (mLs) & Net Wt\# Found (gms) & Laboratory \# \\
\hline Tk-101 & DL & Comp & $12 / 31 / 97$ & 12945 & 250 & 228.1 & S97S000234 \\
Tk-101 & & DL & Comp & $12 / 31 / 97$ & .12946 & 250 & 223.9 & S97S000234 \\
& & & & & & & & Total Weight
\end{tabular}

\begin{tabular}{lllcccccc} 
Tank \# & Core \# & Matrix & Segment \# & Date & Jar/Vial \# & Vial Size (mLs) & Net Wt Found (gms) & Laboratory \# \\
\hline TX-107 & R9A & Sludge & Aug-1 & $1 / 24 / 96$ & 9166 & $\begin{array}{c}60 \\
\text { Total Weight }\end{array}$ & $\frac{13.9}{13.9}$ & S96T000395
\end{tabular}

\begin{tabular}{|c|c|c|c|c|c|c|c|c|}
\hline Tank \# & Core \# & Matrix & Segment \# & Date & Jar/Vial \# & Vial Size (mLs) & Net Wt\# Found (gms) & Laboratory \# \\
\hline TX-244 & Grab & GL & & $1 / 30 / 96$ & 9219 & 60 & 55.7 & S96T000275 \\
\hline $\mathrm{TX}-244$ & Grab & GL & & $1 / 30 / 96$ & 9220 & 60 & 73.9 & S96T000275 \\
\hline TX-244 & Grab & GL & & $2 / 2 / 96$ & 9351 & 20 & 14.8 & S95T003767 \\
\hline TX-244 & Grab & GL & $97-1$ & $9 / 11 / 97$ & 13262 & 40 & 44.6 & S97T001211 \\
\hline $\mathrm{TX}-244$ & Grab & GL & $97-2$ & $9 / 11 / 97$ & 13263 & 40 & 44.7 & S97T001212 \\
\hline TX-244 & Grab & GL & $97-3$ & 9/11/97 & 13264 & $\begin{array}{c}40 \\
\text { Total Weight }\end{array}$ & $\frac{44.7}{278.4}$ & S97T001213 \\
\hline
\end{tabular}

\begin{tabular}{|c|c|c|c|c|c|c|c|c|}
\hline Tank\# & Core\# & Matrix & Segment \# & Date & Jar/Vial \# & Vial Size (mLs) & Net Wt\# Found (gms) & Laboratory \# \\
\hline TY-104 & $\mathbf{R}$ & Sludge & Aug-9 & $4 / 28 / 95$ & 7159 & $\begin{array}{c}40 \\
\text { Total Weight }\end{array}$ & $\begin{array}{r}19.1 \\
19.1\end{array}$ & S95T000317 \\
\hline $\begin{array}{l}\text { TY-104 } \\
\text { TY-104 } \\
\text { TY-104 }\end{array}$ & $\begin{array}{l}\text { R15 } \\
\text { R15 } \\
\text { R15 }\end{array}$ & $\begin{array}{l}\text { Sludge } \\
\text { Sludge } \\
\text { Sludge }\end{array}$ & $\begin{array}{l}\text { Aug-9 } \\
\text { Auger } \\
\text { Auger }\end{array}$ & $\begin{array}{r}3 / 15 / 95 \\
3 / 3 / 95 \\
3 / 3 / 95\end{array}$ & $\begin{array}{l}6424 \\
6479 \\
6560\end{array}$ & $\begin{array}{c}40 \\
125 \\
125 \\
\text { Total Weight }\end{array}$ & $\begin{array}{c}18.7 \\
42 \\
13.4 \\
74.1\end{array}$ & S95T000234 \\
\hline
\end{tabular}


Vials in 11A1 and 11A2

\begin{tabular}{|c|c|c|c|c|c|c|c|c|}
\hline Tank\# & Core \# & Matrix & Segment \# & Date & Jar/Vial \# & Vial Size (mLs) & Net Wt\# Found (gms) & Laboratory \# \\
\hline TY-106 & R6 & Sludge & Aug-11 & $3 / 20 / 95$ & 6430 & 40 & 40.33 & \multirow{3}{*}{ S95T000297 } \\
\hline \multirow[t]{2}{*}{ TY-106 } & R6 & Sludge & Auger & $3 / 8 / 95$ & 6480 & 125 & 14.1 & \\
\hline & & & & & & Total Weight & 54.43 & \\
\hline \multirow[t]{2}{*}{ TY-106 } & \multirow[t]{2}{*}{ R7 } & \multirow[t]{2}{*}{ Sludge } & \multirow[t]{2}{*}{ Aug-10 } & \multirow[t]{2}{*}{$3 / 20 / 95$} & \multirow[t]{2}{*}{6429} & 40 & \multirow{2}{*}{$\begin{array}{r}51.61 \\
51.61\end{array}$} & \\
\hline & & & & & & Total Weight & & \\
\hline Tank \# & Core \# & Matrix & Segment \# & Date & Jar/Vial \# & Vial Size (mLs) & Net Wt\# Found (gms) & Laboratory \# \\
\hline $\mathrm{U}-10 \mathrm{I}$ & Grab & GL & 1 & $9 / 2 / 97$ & 13241 & 40 & 47.2 & S96T003477 \\
\hline U-101 & Grab & GL & 2 & & U101-2 & 125 & 137 & \\
\hline U-101 & Grab & $\overline{G L}$ & 2 & $9 / 2 / 97$ & 13240 & 40 & 47.6 & S96T003478 \\
\hline U-101 & Grab & GL & 3 & $9 / 2 / 97$ & 13242 & 40 & 47.6 & S96T003479 \\
\hline U-101 & Grab & GL & 4 & $9 / 2 / 97$ & 13243 & 40 & 47.1 & S96T003480 \\
\hline & & & & & & Total Weight & 326.5 & \\
\hline
\end{tabular}

\begin{tabular}{|c|c|c|c|c|c|c|c|c|}
\hline Tank \# & Core\# & Matrix & Segment \# & Date & Jar/Vial \# & Vial Size (mLs) & Net Wt\# Found (gms) & Laboratory \# \\
\hline $\mathrm{U}-102$ & 143 & $\mathrm{DL}$ & 1 & $5 / 4 / 96$ & 8455 & $\begin{array}{c}40 \\
\text { Total Weight }\end{array}$ & $\frac{32.7}{32.7}$ & S96T002324 \\
\hline U-102 & 143 & $\mathrm{LL}$ & 5 & $4 / 29 / 96$ & 9851 & $\begin{array}{l}125 \\
\text { Total Weight }\end{array}$ & $\frac{109.4}{109.4}$ & \\
\hline$U-102$ & 143 & Salt & 5 & $5 / 28 / 96$ & 10209 & 60 & 46 & $\mathrm{~S} 96 \mathrm{~T} 002502$ \\
\hline U-102 & 143 & Salt & 5 & $9 / 17 / 97$ & 13476 & 60 & 35.4 & S96T002664 \\
\hline U-102 & 143 & Salt & $5 \mathrm{~A}$ & $5 / 28 / 96$ & 10206 & 60 & 118.1 & S96T002503 \\
\hline U-102 & 143 & Salt & $5 \mathrm{~B}$ & $5 / 28 / 96$ & 10204 & 60 & 83 & S96T002669 \\
\hline $\mathrm{U}-102$ & 143 & Salt & $5 \mathrm{~B}$ & $5 / 28 / 96$ & 10205 & 60 & 97.4 & S96T002670 \\
\hline U-102 & 143 & Salt & 6 & $5 / 13 / 96$ & 10043 & 250 & 107.2 & S96T002754 \\
\hline$U-102$ & 143 & Sait & 6 & $5 / 29 / 96$ & 10230 & 60 & 114.6 & S96T002756 \\
\hline \multirow[t]{2}{*}{ U- 102} & 143 & Salt & Comp & $7 / 22 / 96$ & 10672 & 60 & 52.3 & S96T003679 \\
\hline & & & & & & Total Weight & 654 & \\
\hline U-102 & 143 & Sludge & 1 & $4 / 24 / 96$ & 9843 & 250 & 55 & S96T002325 \\
\hline U-102 & 143 & Sludge & 1 & $5 / 4 / 96$ & 9890 & 60 & 77 & S96T002327 \\
\hline$U-102$ & 143 & Sludge & 1 & $5 / 4 / 96$ & 9891 & 60 & 13.6 & S96T002329 \\
\hline
\end{tabular}

17-Mar-98 
Vials in 11A1 and 11A2

\begin{tabular}{|c|c|c|c|c|c|c|c|c|}
\hline Tank \# & Core \# & Matrix & Segment \# & Date & Jar/Vial \# & Vial Size (mLs) & Net Wt\# Found (gms) & Laboratory \# \\
\hline U-102 & 143 & Sludge & 2 & $5 / 4 / 96$ & 9892 & 60 & 49.8 & S96T002333 \\
\hline $\mathbf{U}-102$ & 143 & Sludge & 3 & $5 / 4 / 96$ & 9894 & 60 & 74.1 & S96T002339 \\
\hline U-102 & 143 & Sludge & 3 & $5 / 4 / 96$ & 9895 & 60 & 78.8 & S96T002342 \\
\hline U-102 & 143 & Sludge & 4 & $4 / 24 / 96$ & 9840 & 250 & 98.3 & S96T002343 \\
\hline U-102 & 143 & Sludge & 4 & $4 / 24 / 96$ & 9841 & 250 & 84 & \$96T002346 \\
\hline U-102 & 143 & Sludge & 4 & $5 / 4 / 96$ & 9896 & 60 & 68 & S96T002345 \\
\hline U-102 & 143 & Sludge & 4 & $7 / 10 / 96$ & 9897 & $\begin{array}{c}60 \\
\text { Total Weight }\end{array}$ & $\frac{79.9}{678.5}$ & S96T002348 \\
\hline $\mathrm{U}-102$ & 144 & DL & 1 & $5 / 6 / 96$ & 10013 & 250 & 216.7 & S96T002548 \\
\hline U-102 & 144 & $\mathrm{DL}$ & 1 & $5 / 30 / 96$ & 10231 & $\begin{array}{c}60 \\
\text { Total Weight }\end{array}$ & $\frac{41.1}{257.8}$ & \$96T002550 \\
\hline U-102 & 144 & FB & Fblk & $5 / 13 / 96$ & 10050 & 250 & 115.2 & S96T002760 \\
\hline U.102 & 144 & FB & Fblk & $5 / 30 / 96$ & 9918 & $\begin{array}{c}40 \\
\text { Total Weight }\end{array}$ & $\frac{40.4}{155.6}$ & S96T002761 \\
\hline U-102 & 144 & Salt & 1 & $5 / 30 / 96$ & 10232 & 60 & 39 & \$96T002637 \\
\hline $\mathrm{U}-102$ & 144 & Salt & 2 & $5 / 6 / 96$ & 10024 & 125 & 47 & S96T002552 \\
\hline U-102 & 144 & Salt & 2 & $5 / 30 / 96$ & 10233 & 60 & 100.1 & S96T002638 \\
\hline U-102 & 144 & Salt . & 2 & $5 / 30 / 96$ & 10235 & 60 & 109.7 & \$96T002639 \\
\hline U-102 & 144 & Salt & 3 & $5 / 30 / 96$ & 10236 & 60 & 104.9 & S96T002640 \\
\hline U-102 & 144 & Salt & 3 & $5 / 30 / 96$ & 10238 & 60 & 84.6 & S96T002641 \\
\hline U-102 & 144 & Salt & 4 & $5 / 13 / 96$ & 10035 & 250 & 74.3 & S96T002782 \\
\hline U-102 & 144 & Salt & 4 & $5 / 13 / 96$ & 10039 & 250 & 86.2 & S96T002781 \\
\hline U.102 & 144 & Salt & 4 & $5 / 30 / 96$ & 10253 & 60 & 99.8 & S96T002787 \\
\hline U-102 & 144 & Salt & 4 & $5 / 30 / 96$ & 10254 & 60 & 99.1 & S96T002788 \\
\hline U-102 & 144 & Salt & 5 & $5 / 13 / 96$ & 10089 & 250 & 79.8 & \$96T002784 \\
\hline U-102 & 144 & Salt & 5 & $5 / 30 / 96$ & 10255 & 60 & 100 & \$96T002789 \\
\hline $\mathrm{U}-102$ & 144 & Salt & 5 & $5 / 30 / 96$ & 10256 & 60 & 98.7 & S967002790 \\
\hline $\mathrm{U}-102$ & 144 & Salt & 6 & $5 / 30 / 96$ & 10257 & 60 & 61.1 & S96T002791 \\
\hline U-102 & 144 & Salt & 6 & $5 / 30 / 96$ & 10258 & 60 & 102.7 & S96T002792 \\
\hline U-102 & 144 & Salt & $6 A$ & $5 / 14 / 96$ & 9917 & 60 & 15.8 & S96T002845 \\
\hline U-102 & 144 & Salt & Comp & $7 / 23 / 96$ & 10673 & 60 & 73.4 & S96T003674 \\
\hline & & & & & & Total Weight & 1376.2 & \\
\hline
\end{tabular}


Vials in 11A1 and 11A2

\begin{tabular}{|c|c|c|c|c|c|c|c|c|}
\hline Tank \# & Core\# & Matrix & Segment \# & Date & Jar/Vial \# & Vial Size (mLs) & Net Wt\# Found (gms) & Laboratory \# \\
\hline U-103 & 175 & DL & 1 & $11 / 8 / 96$ & 11772 & $\begin{array}{l}60 \\
\text { Total Weight }\end{array}$ & $\frac{84.3}{84.3}$ & S96T005799 \\
\hline $\begin{array}{l}\text { U-103 } \\
\text { U-103 } \\
\text { U-103 }\end{array}$ & $\begin{array}{l}175 \\
175 \\
175\end{array}$ & $\begin{array}{l}\text { LL } \\
\mathbf{L L} \\
\mathbf{L L}\end{array}$ & $\begin{array}{c}2 \\
2 \mathrm{~A} \\
2 \mathrm{~B}\end{array}$ & $\begin{array}{r}10 / 30 / 96 \\
11 / 8 / 96 \\
11 / 8 / 96\end{array}$ & $\begin{array}{l}11627 \\
11773 \\
11774\end{array}$ & $\begin{array}{c}60 \\
60 \\
60 \\
\text { Total Weight }\end{array}$ & $\begin{array}{r}56.9 \\
63.1 \\
48.5 \\
168.5\end{array}$ & $\begin{array}{l}\text { S97T005804 } \\
\text { S96T005805 } \\
\text { S96T005806 }\end{array}$ \\
\hline $\begin{array}{l}\text { U-103 } \\
\text { U-103 } \\
\text { U-103 } \\
\text { U-103 }\end{array}$ & $\begin{array}{l}175 \\
175 \\
175 \\
175\end{array}$ & $\begin{array}{l}\text { Salt } \\
\text { Salt } \\
\text { Salt } \\
\text { Sait }\end{array}$ & $\begin{array}{c}1 \\
2 \\
2 \mathrm{~A} \\
2 \mathrm{R}\end{array}$ & $\begin{array}{r}10 / 29 / 96 \\
10 / 30 / 96 \\
10 / 30 / 96 \\
1 / 29 / 97\end{array}$ & $\begin{array}{l}11699 \\
11540 \\
11633 \\
11637\end{array}$ & $\begin{array}{c}125 \\
250 \\
60 \\
60 \\
\text { Total Weight }\end{array}$ & $\begin{array}{c}60.4 \\
176 \\
32.1 \\
76.8 \\
345.3\end{array}$ & $\begin{array}{l}\text { S96T005794 } \\
\text { S96T005795 } \\
\text { S96T005796 } \\
\text { S97T000082 }\end{array}$ \\
\hline $\begin{array}{l}\text { U-103 } \\
\text { U-103 } \\
\text { U-103 }\end{array}$ & $\begin{array}{l}176 \\
176 \\
176\end{array}$ & $\begin{array}{l}\text { DL } \\
\text { DL } \\
\text { DL }\end{array}$ & $\begin{array}{l}1 \\
1 \\
3\end{array}$ & $\begin{array}{l}1 / 29 / 97 \\
1 / 31 / 97 \\
1 / 31 / 97\end{array}$ & $\begin{array}{l}11935 \\
12080 \\
12082\end{array}$ & $\begin{array}{c}250 \\
60 \\
60 \\
\text { Total Weight }\end{array}$ & $\begin{array}{c}141.1 \\
96.3 \\
96.4 \\
333.8\end{array}$ & $\begin{array}{l}\text { S97Tr000049 } \\
\text { S97T000078 } \\
\text { S97T000079 }\end{array}$ \\
\hline $\begin{array}{l}\text { U-103 } \\
\text { U-103 }\end{array}$ & $\begin{array}{l}176 \\
176\end{array}$ & $\begin{array}{l}\text { FB } \\
\text { FB }\end{array}$ & $\begin{array}{l}\text { Fblk } \\
\text { Fblk }\end{array}$ & $\begin{array}{l}1 / 29 / 97 \\
1 / 31 / 97\end{array}$ & $\begin{array}{l}11932 \\
12078\end{array}$ & $\begin{array}{c}250 \\
60 \\
\text { Total Weight }\end{array}$ & $\begin{array}{c}162.2 \\
60.5 \\
222.7\end{array}$ & $\begin{array}{l}\text { S97T000048 } \\
\text { S97T000080. }\end{array}$ \\
\hline $\begin{array}{l}\text { U-103 } \\
\text { U-103 } \\
\text { U-103 } \\
\text { U-103 } \\
\text { U-103 }\end{array}$ & $\begin{array}{l}176 \\
176 \\
176 \\
176 \\
176\end{array}$ & $\begin{array}{l}\text { Salt } \\
\text { Salt } \\
\text { Salt } \\
\text { Salt } \\
\text { Salt }\end{array}$ & $\begin{array}{l}1 \\
3 \\
4 \\
4 \\
4\end{array}$ & $\begin{array}{l}1 / 29 / 97 \\
1 / 29 / 97 \\
1 / 29 / 97 \\
1 / 31 / 97 \\
1 / 31 / 97\end{array}$ & $\begin{array}{l}11814 \\
12103 \\
11898 \\
12084 \\
12085\end{array}$ & $\begin{array}{c}60 \\
60 \\
125 \\
60 \\
60 \\
\text { Total Weight }\end{array}$ & $\begin{array}{r}36.7 \\
34.5 \\
46.3 \\
99.9 \\
108.3 \\
325.7\end{array}$ & $\begin{array}{l}\text { S97T000072 } \\
\text { S97T000073 } \\
\text { S97T000053 } \\
\text { S97T000074 } \\
\text { S97T000075 }\end{array}$ \\
\hline $\begin{array}{l}U-103 \\
U-103 \\
U-103 \\
U-103 \\
U-103 \\
U-103 \\
U-103\end{array}$ & $\begin{array}{l}176 \\
176 \\
176 \\
176 \\
176 \\
176 \\
176\end{array}$ & $\begin{array}{l}\text { Sludge } \\
\text { Sludge } \\
\text { Sludge } \\
\text { Sludge } \\
\text { Sludge } \\
\text { Sludge } \\
\text { Sludge }\end{array}$ & $\begin{array}{c}5 \text { Rgs } \\
6 \\
6 \\
6 \\
7 \text { Rgs } \\
8 \text { Rgs } \\
9\end{array}$ & $\begin{array}{l}2 / 10 / 97 \\
1 / 29 / 97 \\
1 / 31 / 97 \\
1 / 31 / 97 \\
2 / 25 / 97 \\
2 / 25 / 97 \\
11 / 5 / 97\end{array}$ & $\begin{array}{l}11819 \\
11938 \\
12086 \\
12087 \\
12213 \\
12212 \\
13501\end{array}$ & $\begin{array}{c}60 \\
250 \\
60 \\
60 \\
40 \\
40 \\
60\end{array}$ & $\begin{array}{c}121.9 \\
57.6 \\
109.4 \\
106.3 \\
62.1 \\
65 \\
47\end{array}$ & $\begin{array}{l}\text { S97T000115 } \\
\text { S97T000055 } \\
\text { S97T000076 } \\
\text { S97T000077 } \\
\text { S97T000238 } \\
\text { S97T000239 } \\
\text { S97T000041 }\end{array}$ \\
\hline
\end{tabular}




\section{Vials in 11A1 and 11A2}

\begin{tabular}{|c|c|c|c|c|c|c|c|c|}
\hline Tank \# & Core\# & Matrix & Segment \# & Date & Jar/Vial \# & Vial Size (mLs) & Net Wt\# Found (gms) & \multirow[t]{2}{*}{ Laboratory \# } \\
\hline & & & & & & Total Weight & 569.3 & \\
\hline U-103 & 182 & DL & 8 & $4 / 16 / 97$ & 12188 & 250 & 256.9 & $\$ 97 \mathrm{~T} 000539$ \\
\hline U-103 & 182 & DL & 8 & $4 / 16 / 97$ & 12227 & 40 & 57.6 & S97T000541 \\
\hline U-103 & 182 & DL & 9 & $4 / 14 / 97$ & 12187 & 250 & 99.7 & S97T000540 \\
\hline U-103 & 182 & DL & 9 & $4 / 16 / 97$ & 12226 & 40 & 57.7 & \$97T000542 \\
\hline & & & & & & Total Weight & 471.9 & \\
\hline U-103 & 182 & Salt & 10 & $4 / 16 / 97$ & 12146 & 60 & 105.5 & S97T000544 \\
\hline U-103 & 182 & Salt & 10 & $4 / 16 / 97$ & 12147 & 60 & 95.6 & S97T000554 \\
\hline U-103 & 182 & Salt & 2 & $4 / 8 / 97$ & 11884 & 125 & 34.3 & S97T000479 \\
\hline U-103 & 182 & Salt & 2 & $4 / 8 / 97$ & 11885 & 125 & 50.8 & S97T000476 \\
\hline U-103 & 182 & Salt & 3 & $4 / 8 / 97$ & 12194 & 250 & 57.7 & \$97T000467 \\
\hline $\mathrm{U}-103$ & 182 & Salt & 3 & $4 / 14 / 97$ & 12140 & 60 & 108.2 & \$97T000477 \\
\hline U.103 & 182 & Salt & 3 & $4 / 15 / 97$ & 12144 & 60 & 54.4 & S97T000480 \\
\hline U-103 & 182 & Salt & 5 & $4 / 15 / 97$ & 12137 & 60 & 77.7 & S97T000513 \\
\hline U-103 & 182 & Salt & 5 & $4 / 15 / 97$ & 12305 & 60 & 107.4 & \$97T000510 \\
\hline U-103 & 182 & Salt & 6 & $4 / 15 / 97$ & 12138 & 60 & 75.4 & $\$ 97 T 000511$ \\
\hline U-103 & 182 & Salt & 6 & $4 / 15 / 97$ & 12139 & 60 & 107.4 & S97T000514 \\
\hline U-103 & 182 & Salt & 7 & $4 / 9 / 97$ & 12191 & 250 & 52.7 & S97T000502 \\
\hline U-103 & 182 & Salt & 7 & $4 / 9 / 97$ & 12201 & 250 & 44.9 & S97T000501 \\
\hline U-103 & 182 & Salt & 7 & $4 / 16 / 97$ & 12141 & 60 & 105.1 & S97T000512 \\
\hline U-103 & 182 & Salt & 7 & $4 / 16 / 97$ & 12142 & 60 & 91.9 & S97T000515 \\
\hline U-103 & 182 & Salt & 9 & $4 / 14 / 97$ & 12190 & 250 & 88.4 & S97T000547 \\
\hline U-103 & 182 & Salt & 9 & $4 / 16 / 97$ & 12143 & 60 & 107.4 & S97T000553 \\
\hline & & & & & & Total Weight & 1364.8 & \\
\hline U-103 & 182 & Sludge & 1 & $4 / 8 / 97$ & 11876 & 125 & 27.4 & S97T000465 \\
\hline U-103 & 182 & Sludge & 1 & $4 / 8 / 97$ & 11878 & 125 & 54.2 & S97T000475 \\
\hline U-103 & 182 & Sludge & 4 Rgs & & 12719 & 250 & 77.6 & S97T000493 \\
\hline U. 103 & 182 & Sludge & 4 Rgs & $7 / 11 / 97$ & 13058 & 60 & 24.4 & S97T001716 \\
\hline & & & & & & Total Weight & 183.6 & \\
\hline U.103 & Grab & GL & 1 & $6 / 2 / 95$ & 7267 & 125 & 111.2 & S95T000969 \\
\hline U-103 & Grab & GL & 3 & $6 / 2 / 95$ & 7260 & 125 & 179.2 & S95T000967 \\
\hline & & & & & & Total Weight & 290.4 & \\
\hline
\end{tabular}


Vials in 11A1 and 11A2

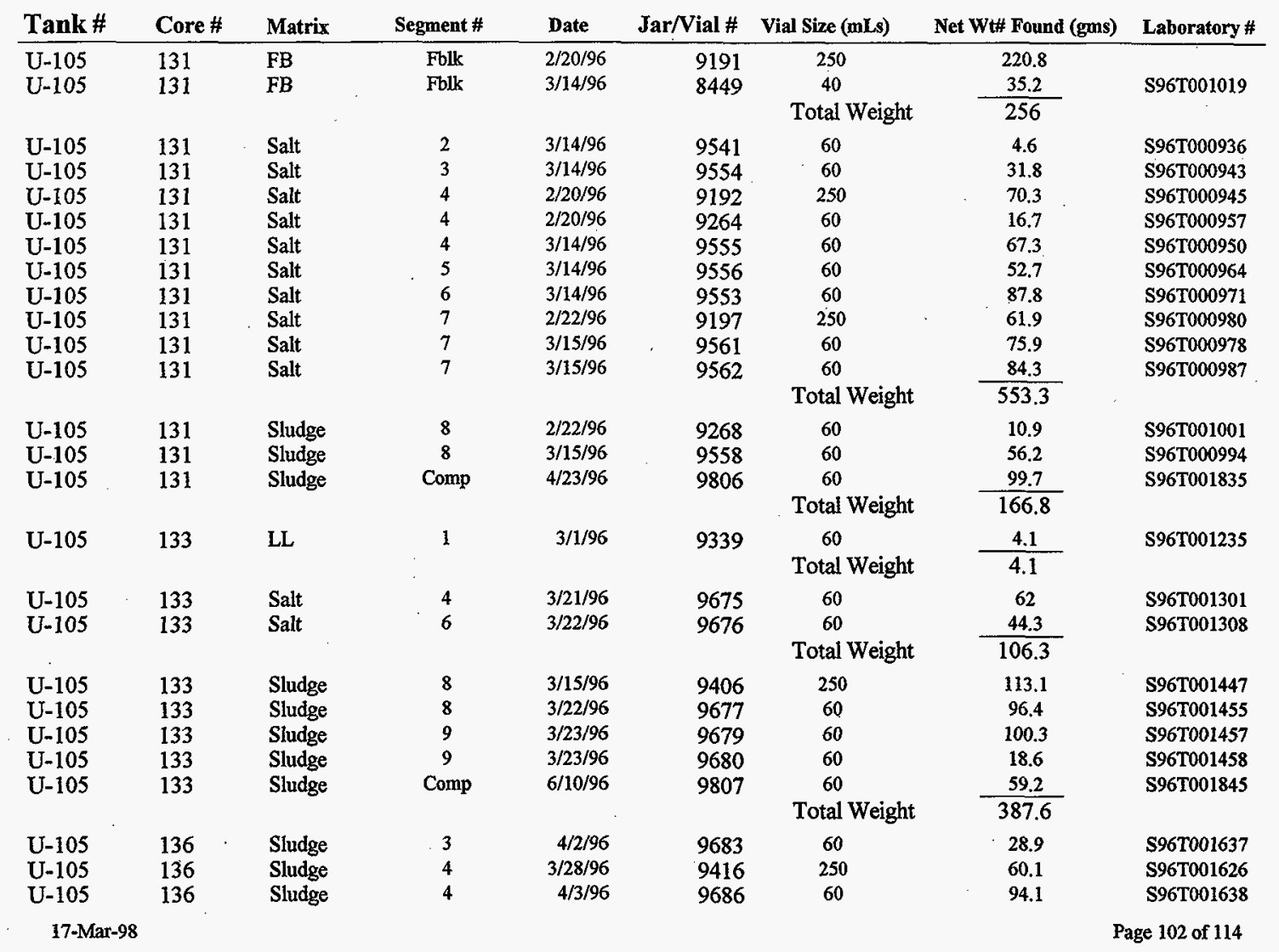


Vials in 11A1 and 11A2

\begin{tabular}{|c|c|c|c|c|c|c|c|c|}
\hline Tank\# & Core\# & Matrix & Segment \# & Date & Jar/Vial \# & Vial Size (mLs) & Net Wt\# Found (gms) & Laboratory \# \\
\hline $\mathrm{U}-105$ & 136 & Sludge & 4 & $4 / 3 / 96$ & 9689 & 60 & 96 & S96T001639 \\
\hline U-105 & 136 & Sludge & 4 & $9 / 23 / 97$ & 13455 & 60 & 26 & \\
\hline$U-105$ & 136 & Sludge & 5 & $3 / 28 / 96$ & 9422 & 250 & 74.6 & S96T001628 \\
\hline U-105 & 136 & Sludge & 5 & $4 / 3 / 96$ & 9690 & 60 & 86.7 & S96T001640 \\
\hline U-105 & 136 & Sludge & 5 & $4 / 3 / 96$ & 9691 & 60 & 89.5 & S96T001641 \\
\hline U-105 & 136 & Sludge & 6 & $4 / 4 / 96$ & 9703 & 60 & 15.8 & S96T001642 \\
\hline U-105 & 136 & Sludge & 7 & $4 / 3 / 96$ & 9477 & 250 & 88.2 & S96T001706 \\
\hline U-105 & 136 & Sludge & 7 & $4 / 3 / 96$ & 9481 & 250 & 89.5 & \$96T001707 \\
\hline U-105 & 136 & Sludge & 7 & $4 / 4 / 96$ & 9692 & 60 & 59.4 & S96T001711 \\
\hline$U-105$ & 136 & Sludge & 7 & $4 / 4 / 96$ & 9704 & 60 & 82.4 & S96T001712 \\
\hline U-105 & 136 & Sludge & 9 & $4 / 3 / 96$ & 9475 & 250 & 183.5 & S96T001736 \\
\hline U-105 & 136 & Sludge & 9 & $4 / 5 / 96$ & 9712 & 60 & 73.2 & S96T001740 \\
\hline U-105 & 136 & Sludge & $9 \mathrm{~A}$ & $4 / 3 / 96$ & 9480 & 250 & 183.2 & S96T001761 \\
\hline U-105 & 136 & Sludge & $9 \mathrm{~A}$ & $4 / 3 / 96$ & 9702 & 60 & 12.1 & S96T001737 \\
\hline U-105 & 136 & Sludge & $9 \mathrm{~A}$ & $4 / 5 / 96$ & 9711 & 60 & 79.9 & S96T001765 \\
\hline U-105 & 136 & Sludge & Comp & $4 / 8 / 96$ & 9808 & 60 & 120.9 & S96T001853 \\
\hline & & & & & & Total Weigh & 1544 & \\
\hline
\end{tabular}

Tank \# Core \# Matrix Segment\# Date Jar/Vial \# Vial Size (mLs) Net Wt\# Found (gms) Laboratory \#

\begin{tabular}{|c|c|c|c|c|c|c|c|c|}
\hline U-106 & 147 & DL & 1 & $5 / 21 / 96$ & 10090 & 250 & 210.3 & $\mathrm{~S} 96 \mathrm{~T} 002983$ \\
\hline U-106 & 147 & $\mathrm{DL}$ & 1 & $6 / 20 / 96$ & 10312 & 60 & 89.7 & S96T003061 \\
\hline U-106 & 147 & DL & 2 & $5 / 21 / 96$ & 10061 & 125 & 34.7 & S96T002988 \\
\hline \multirow[t]{2}{*}{ U-106 } & 147 & $\mathrm{DL}$ & 2 & $6 / 20 / 96$ & 10456 & 60 & 50.4 & $\mathrm{~S} 96 \mathrm{~T} 003062$ \\
\hline & & & & & & Total Weight & 385.1 & \\
\hline \multirow[t]{2}{*}{ U-106 } & 147 & Salt & 5 & $6 / 21 / 96$ & 10462 & 60 & 5.7 & S96T003078 \\
\hline & & & & & & Total Weight & 5.7 & \\
\hline U-106 & 147 & Sludge & 2 & $5 / 21 / 96$ & 10201 & 250 & 103.4 & S96T002984 \\
\hline U-106 & 147 & Sludge & 2 & $6 / 20 / 96$ & 10313 & 60 & 18.5 & S96T003072 \\
\hline U-106 & 147 & Sludge & 3 & $5 / 20 / 96$ & 10190 & 250 & 74.2 & S96T003073 \\
\hline U-106 & 147 & Sludge & 3 & $5 / 20 / 96$ & 10191 & 250 & 98.2 & \$96T002949 \\
\hline U-106 & 147 & Sludge & 3 & $6 / 20 / 96$ & 10457 & 60 & 40.4 & S96T003073 \\
\hline U-106 & 147 & Sludge & 3 & $6 / 21 / 96$ & 10458 & 60 & 73.5 & S96T003074 \\
\hline
\end{tabular}


Vials in 11A1 and 11A2

\begin{tabular}{|c|c|c|c|c|c|c|c|c|}
\hline Tank \# & Core \# & Matrix & Segment \# & Date & Jar/Vial \# & Vial Size (mLs) & Net Wt\# Found (gms) & Laboratory \# \\
\hline U-106 & 147 & Sludge & 4 & $5 / 21 / 96$ & 10194. & 250 & 103.1 & S96T002986 \\
\hline U-106 & 147 & Sludge & 4 & $5 / 21 / 96$ & 10199 & 250 & 72.3 & S96T002987 \\
\hline $\mathrm{U}-106$ & 147 & Sludge & 4 & $6 / 21 / 96$ & 10459 & 60 & 82 & S96T003076 \\
\hline $\mathrm{U}-106$ & 147 & Sludge & 4 & $6 / 21 / 96$ & 10460 & 60 & 73.8 & S96T003075 \\
\hline U-106 & 147 & Sludge & 5 & $5 / 20 / 96$ & 10184 & 250 & 78.2 & S96T002969 \\
\hline U-106 & 147 & Sludge & 5 & $6 / 21 / 96$ & 10461 & 60 & 74.8 & S96T003077 \\
\hline \multirow[t]{2}{*}{ U-106 } & 147 & Sludge & Comp & $7 / 29 / 96$ & 10783 & 60 & 92.4 & \$96T003877 \\
\hline & & & & & & Total Weight & 984.8 & \\
\hline \multirow[t]{2}{*}{ U-106 } & 148 & DL & 1 & $6 / 22 / 96$ & 9922 & 40 & 52.4 & S96T002861 \\
\hline & & & & & & Total Weight & 52.4 & \\
\hline \multirow[t]{2}{*}{ U-106 } & 148 & Salt & 5 & $6 / 23 / 96$ & 10470 & 60 & 57.8 & S96T003025 \\
\hline & & & & & & Total Weight & 57.8 & \\
\hline U-106 & 148 & Sludge & 4 & $5 / 20 / 96$ & 10187 & 250 & 125.7 & S96T002975 \\
\hline U-106 & 148 & Sludge & 4 & $6 / 23 / 96$ & 10467 & 60 & 70.5 & S96T003022 \\
\hline U-106 & 148 & Sludge & 5 & 6/23/96 & 10469 & 60 & 86.5 & S96T003024 \\
\hline \multirow{2}{*}{ U-106 } & 148 & Sludge & Comp & $7 / 31 / 96$ & 10834 & 60 & 88.5 & S96T003883 \\
\hline & & & & & & Total Weight & 371.2 & \\
\hline U-106 & Grab & GL & 3 & & $\mathrm{U} 106-3$ & 125 & 61.4 & \\
\hline U-106 & Grab & GL & 4. & & U106-4 & 125 & 153.5 & \\
\hline U-106 & Grab & GL & 5 & & U106-5 & 125 & 50.1 & \\
\hline \multirow[t]{2}{*}{ U-106 } & Grab & GL & 6 & & U106-6 & 125 & 160.3 & \\
\hline & & & & & 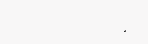 & Total Weight & 425.3 & \\
\hline Tank \# & Core \# & Matrix & Segment \# & Date & Jar/Vial \# & Vial Size (mLs) & Net Wt\# Found (gms) & Laboratory \# \\
\hline U-107 & 129 & $\mathrm{DL}$ & 1 & $2 / 13 / 96$ & 8895 & 250 & 141.8 & S96T000664 \\
\hline U-107 & 129 & DL & 1 & $2 / 15 / 96$ & 8430 & 40 & 59.4 & S96T000636 \\
\hline \multirow[t]{2}{*}{ U-107 } & 129 & DL & 2 & $2 / 21 / 96$ & 9266 & 60 & 78.1 & S96T000640 \\
\hline & & & & & & Total Weight & 279.3 & \\
\hline U-107 & 129 & Salt & 2 & $2 / 13 / 96$ & 9167 & 60 & 11.9 & S96T000646 \\
\hline U-107 & 129 & Salt & 2 & $2 / 13 / 96$ & 9285 & 60 & 34.5 & S96T000643 \\
\hline U-107 & 129 & Salt & 3 & $2 / 13 / 96$ & 8598 & 125 & 25.5 & S96T000648 \\
\hline
\end{tabular}

17-Mar-98

Page 104 of 114 
Vials in 11A1 and 11A2

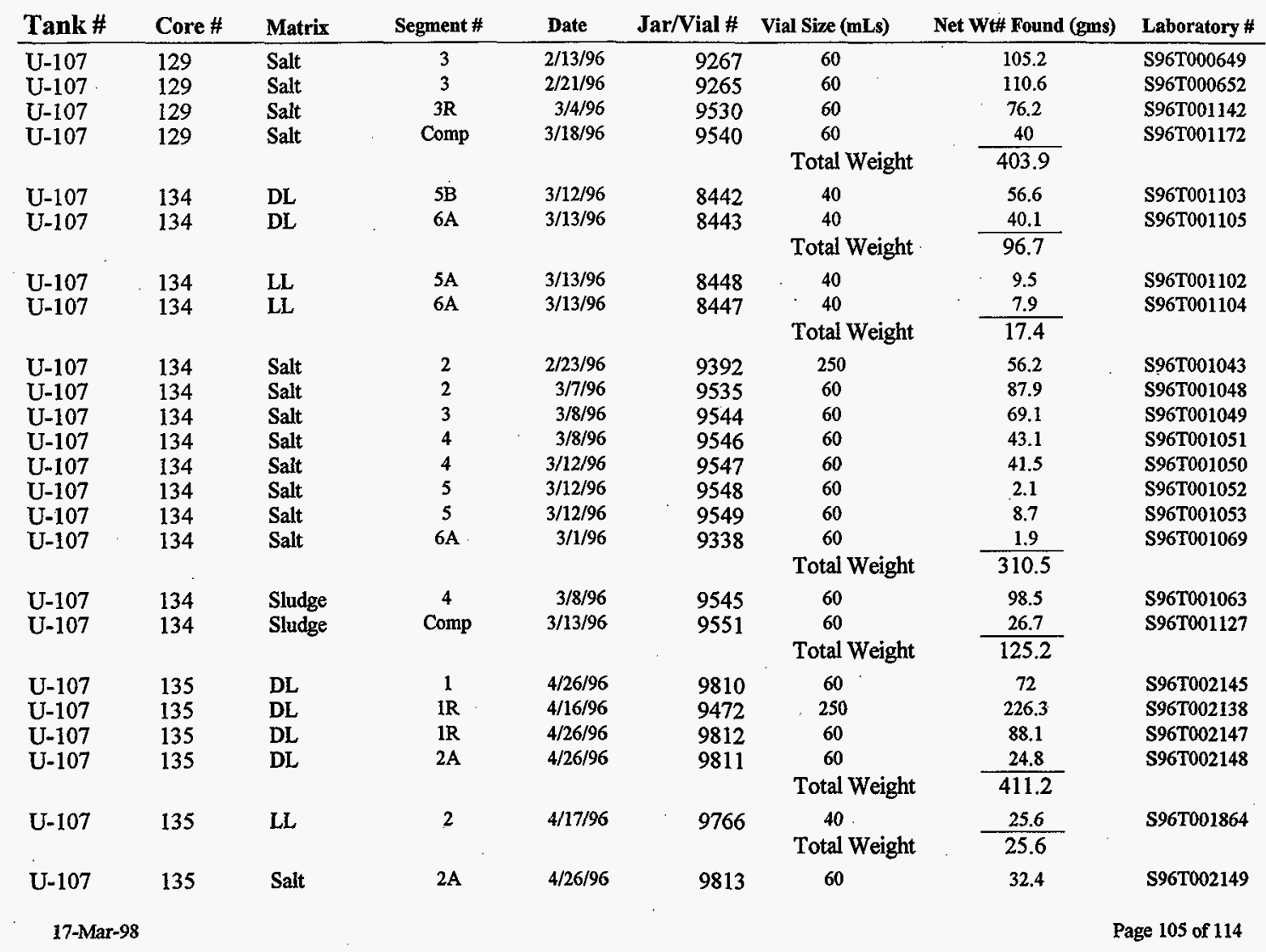


Vials in 11A1 and 11A2

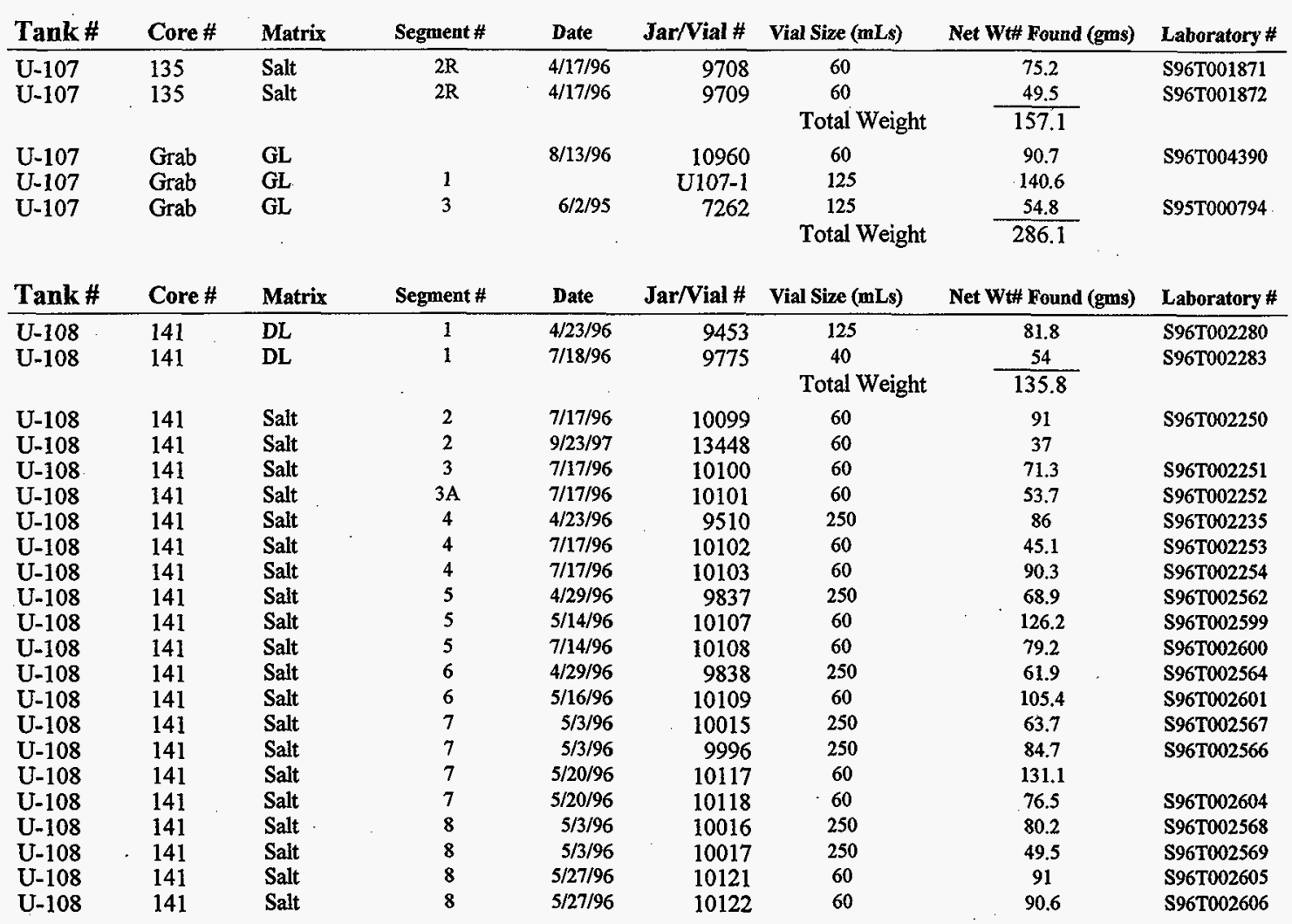


Vials in 11A1 and 11A2

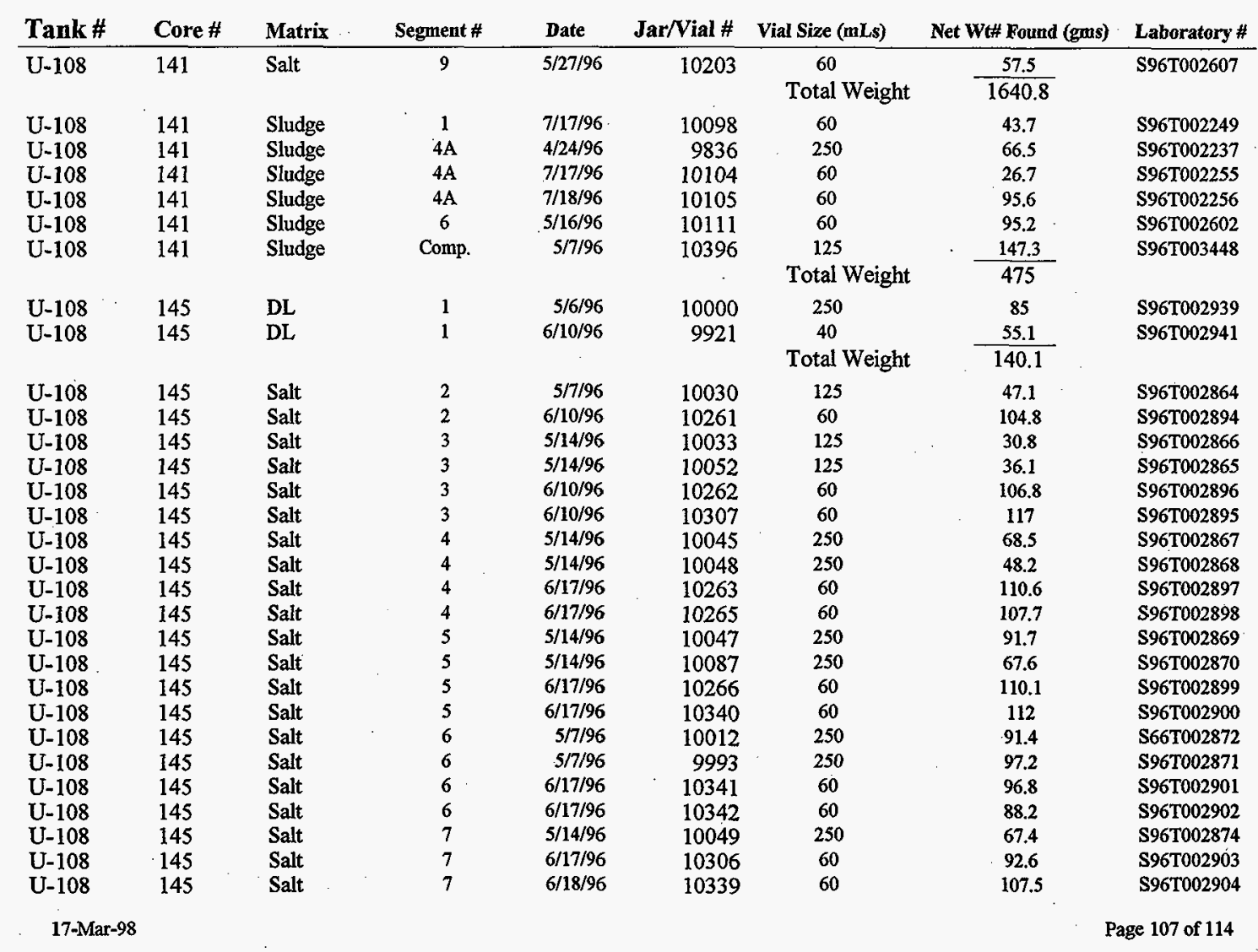


Vials in 11A1 and 11A2

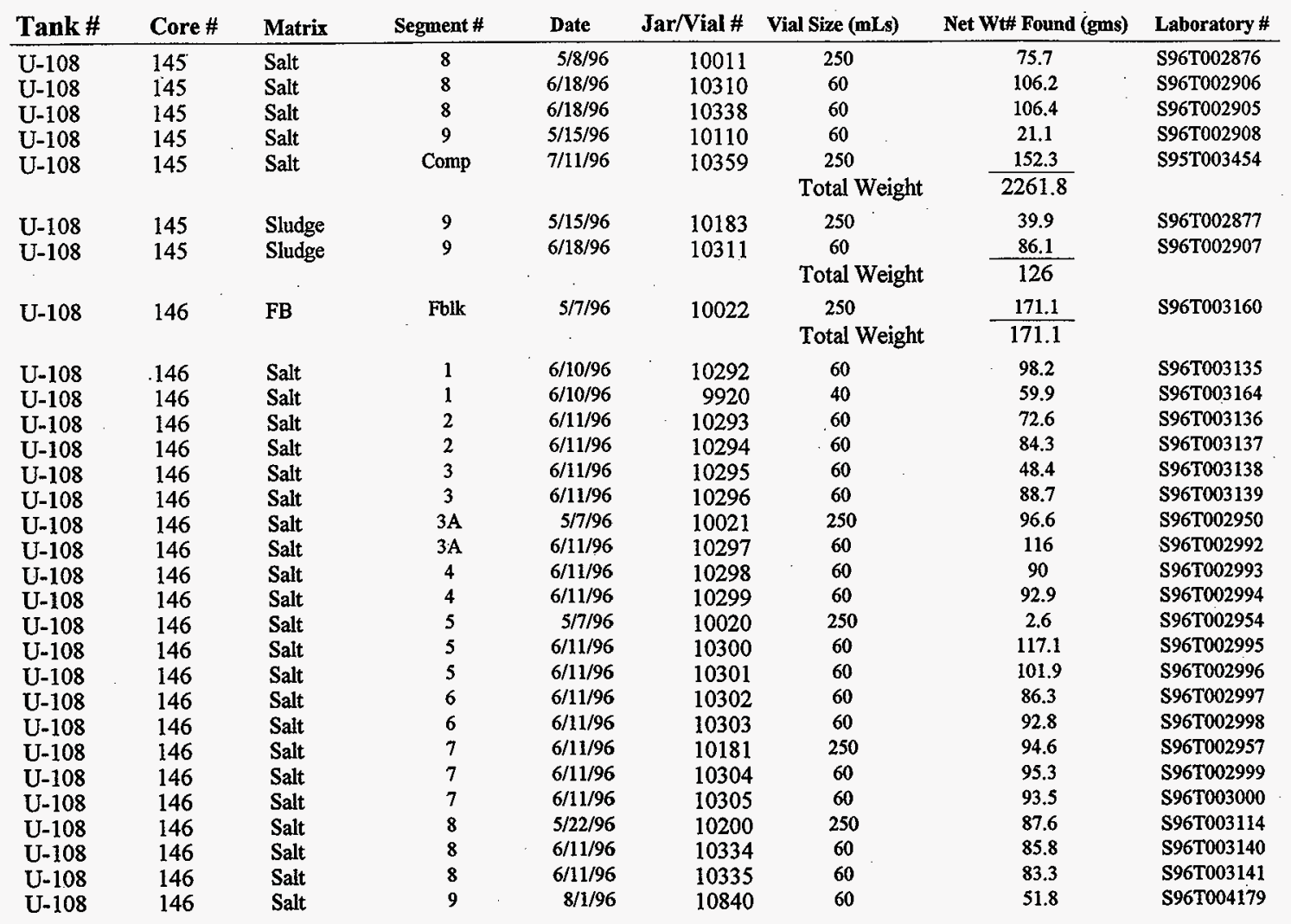


Vials in 11A1 and 11A2

\begin{tabular}{|c|c|c|c|c|c|c|c|c|}
\hline Tank \# & Core \# & Matrix & Segment \# & Date & Jar/Vial \# & Vial Size (mLs) & Net Wt\# Found (gms) & Laboratory \# \\
\hline U-108 & 146 & Salt & Comp & $8 / 1 / 96$ & 10570 & $\begin{array}{l}250 \\
\text { Total Weight }\end{array}$ & $\frac{192.2}{2032.4}$ & S96T004197 \\
\hline U-108 & 146 & Sludge & 9 & $6 / 12 / 96$ & 10336 & $\begin{array}{c}60 \\
\text { Total Weight }\end{array}$ & $\frac{54}{54}$ & S96T003142 \\
\hline $\begin{array}{l}U-108 \\
U-108 \\
U-108\end{array}$ & $\begin{array}{l}\text { Grab } \\
\text { Grab } \\
\text { Grab }\end{array}$ & $\begin{array}{l}\text { GL } \\
\text { GL } \\
\text { GL }\end{array}$ & $\begin{array}{l}2 \\
3\end{array}$ & $\begin{array}{l}6 / 2 / 95 \\
6 / 2 / 95\end{array}$ & $\begin{array}{r}7266 \\
7263 \\
\text { U108-3 }\end{array}$ & $\begin{array}{c}125 \\
125 \\
125 \\
\text { Total Weight }\end{array}$ & $\begin{array}{c}132.3 \\
29.2 \\
181.9 \\
343.4\end{array}$ & $\begin{array}{l}\text { S95T001003 } \\
\text { S95T000979 }\end{array}$ \\
\hline
\end{tabular}

\begin{tabular}{|c|c|c|c|c|c|c|c|c|}
\hline Tank\# & Core \# & Matrix & Segment \# & Date & Jar/Vial \# & Vial Size (mLs) & Net Wt\# Found (gms) & Laboratory \# \\
\hline $\mathrm{U}-109$ & 123 & Salt & 1 & $1 / 15 / 96$ & 9025 & 60 & 14.8 & S96T000192 \\
\hline U-109 & 123 & Salt & 2 & $1 / 15 / 96$ & 9026 & 60 & 82.1 & S96T000193 \\
\hline U-109 & 123 & Salt & 3 & $1 / 15 / 96$ & 9027 & 60 & 62.7 & S96T000194 \\
\hline U-109 & 123 & Salt & $3 \mathrm{~A}$ & $1 / 16 / 96$ & 9069 & 60 & 57.1 & S96T000195 \\
\hline U-109 & 123 & Salt & 4 & $1 / 16 / 96$ & 9070 & 60 & 84.6 & S96T000196 \\
\hline U-109 & 123 & Salt & 5 & $1 / 16 / 96$ & 9071 & 60 & 68.1 & \$96T000197 \\
\hline U-109 & 123 & Salt & 6 & $1 / 16 / 96$ & 9072 & 60 & 109.6 & S96T000199 \\
\hline U-109 & 123 & Salt & 6 & $1 / 16 / 96$ & 9073 & 60 & 90.3 & S96T000198 \\
\hline U-109 & 123 & Salt & 7 & $1 / 16 / 96$ & 9074 & 60 & 52.2 & S96T000201 \\
\hline U-109 & 123 & Salt & 7 & $1 / 16 / 96$ & 9075 & 60 & 103.5 & S96T000200 \\
\hline U-109 & 123 & Salt & 8 & $1 / 17 / 96$ & 9079 & 60 & 100 & \$96T000202 \\
\hline U-109 & 123 & Salt & 8 & $1 / 17 / 96$ & 9080 & 60 & 97.3 & S96T000203 \\
\hline U-109 & 123 & Salt & 9 & $1 / 9 / 96$ & 8852 & 250 & 58.7 & S96T000175 \\
\hline U-109 & 123 & Salt & 9 & $1 / 16 / 96$ & 9076 & 60 & 71.4 & S96T000204 \\
\hline U-109 & 123 & Salt & 9 & $1 / 17 / 96$ & 9077 & 60 & 71.3 & S96T000205 \\
\hline U-109 & 123 & Salt & 9 & $1 / 17 / 96$ & 9078 & 60 & 64.4 & \$96T000206 \\
\hline U-109 & 123 & Salt & Comp & $3 / 14 / 96$ & 9431 & 125 & 76.8 & S96T001379 \\
\hline & & & & & & Total Weight & 1264.9 & \\
\hline U-109 & 124 & $\mathrm{DL}$ & 3 & $1 / 23 / 96$ & 9153 & $\begin{array}{c}60 \\
\text { Total Weight }\end{array}$ & $\frac{56.9}{56.9}$ & S96T000324 \\
\hline U-109 & 124 & FB & Fblk. & $1 / 14 / 96$ & 8836 & 250 & 115 & S96T000118 \\
\hline
\end{tabular}


Vials in 11A1 and 11A2

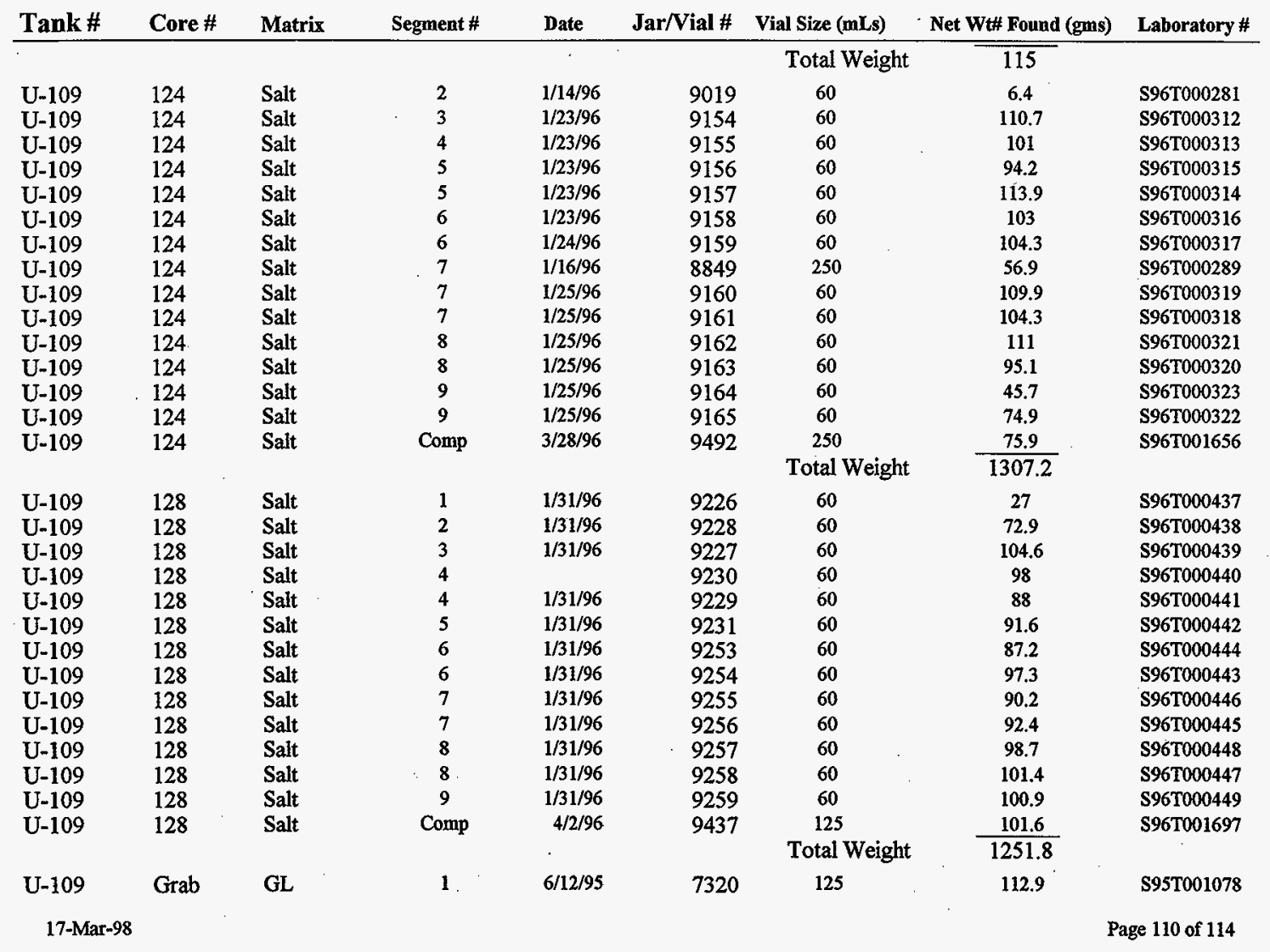


Vials in 11A1 and 11A2

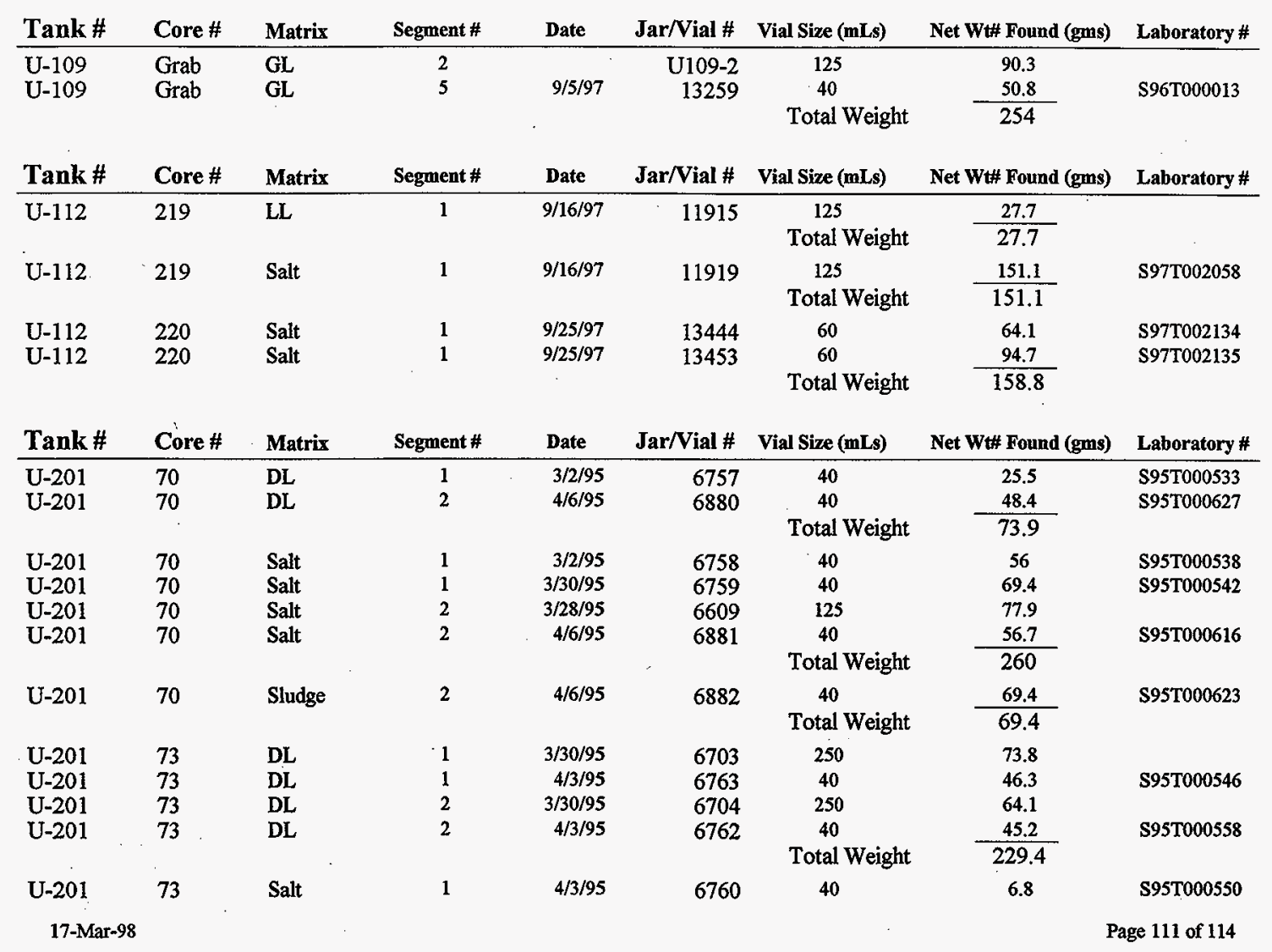


Vials in 11A1 and 11A2

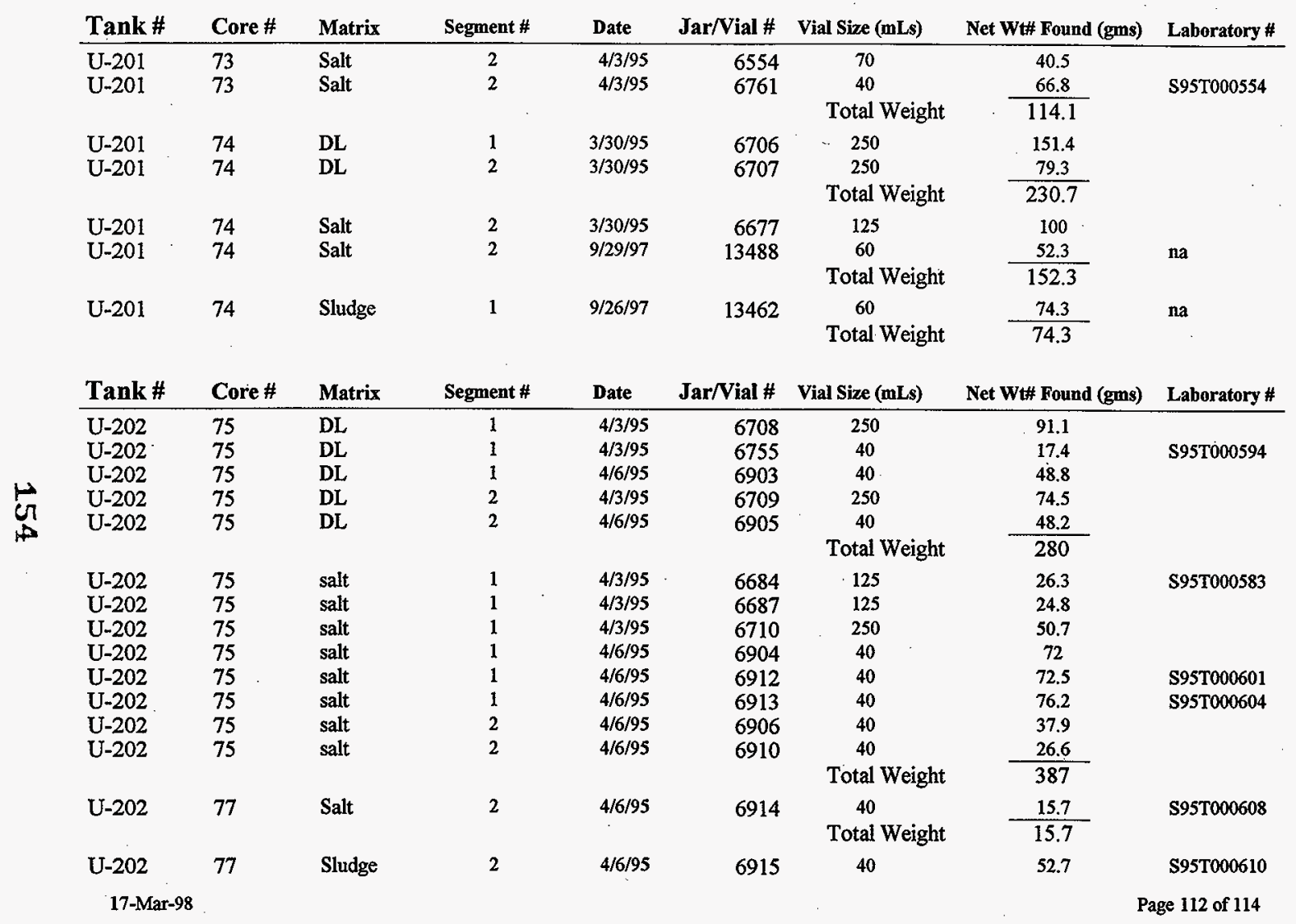




\section{Vials in 11A1 and 11A2}

\begin{tabular}{|c|c|c|c|c|c|c|c|c|}
\hline Tank\# & Core \# & Matrix & Segment \# & Date & Jar/Vial \# & Vial Size (mLs) & Net Wt\# Found (gms) & Laboratory \# \\
\hline & & & & & & Total Weight & 52.7 & \\
\hline U-202 & 78 & DL & 1 & $4 / 4 / 95$ & 6764 & 40 & 33.8 & \\
\hline \multirow{2}{*}{ U-202 } & 78 & DL & 2 & $4 / 4 / 95$ & 6765 & 40 & 11 & \\
\hline & & & & & & Total Weight & 44.8 & \\
\hline \multirow[t]{2}{*}{ U-202 } & 78 & FB & Fblk & $10 / 9 / 95$ & 8025 & 40 & 37 & \\
\hline & & & & & & Total Weight & 37 & \\
\hline \multirow[t]{2}{*}{ U-202 } & 78 & Salt & 2 & $4 / 6 / 95$ & 6917 & 40 & 63 & \\
\hline & & & & & & Total Weight & 63 & \\
\hline U-202 & 78 & Sludge & 1 & $4 / 4 / 95$ & 6692 & 125 & 65.7 & \\
\hline U-202 & 78 & Sludge & 1 & $4 / 6 / 95$ & 6911 & 40 & 75.1 & \\
\hline U-202 & 78 & Sludge & 1 & $4 / 6 / 95$ & 6916 & 40 & 75.2 & \\
\hline $\mathrm{U}-202$ & 78 & Sludge & 1 & 9/17/97 & 13486 & 60 & 31.8 & na \\
\hline \multirow{2}{*}{ U-202 } & 78 & Sludge & 2 & $4 / 6 / 95$ & 6918 & 40 & 73.5 & \\
\hline & & & & & & Total Weight & 321.3 & \\
\hline Tank\# & Core \# & Matrix & Segment \# & Date & Jar/Vial \# & Vial Size (mLs) & Net Wt\# Found (gms) & Laboratory \# \\
\hline U-203 & 79 & $\mathrm{DL}$ & 1 & $4 / 5 / 95$ & 6766 & $\begin{array}{c}40 \\
\text { Total Weight }\end{array}$ & $\frac{23.5}{23.5}$ & S95T000657 \\
\hline & 79 & Salt & 1 & $4 / 5 / 95$ & 6769 & 40 & 57.2 & S95T000683 \\
\hline \multirow{2}{*}{$\begin{array}{l}U-203 \\
U-203\end{array}$} & 79 & Salt & 1 & 9/18/97 & 13483 & 60 & 24.2 & \\
\hline & & & & & & Total Weight & 81.4 & \\
\hline \multirow[t]{2}{*}{ U-203 } & 80 & Salt & 1 & $4 / 6 / 95$ & 6878 & 40 & 74.3 & S95T000691 \\
\hline & & & & & & Total Weight & 74.3 & \\
\hline \multirow[t]{2}{*}{ U-203 } & 80 & Sludge & 1 & $4 / 6 / 95$ & 6879 & 40 & 78.3 & S95T000687 \\
\hline & & & & & & Total Weight & 78.3 & \\
\hline Tank\# & Core \# & Matrix & Segment \# & Date & Jar/Vial \# & Vial Size (mLs) & Net Wt\# Found (gms) & Laboratory \# \\
\hline U-204 & 81 & $\mathrm{DL}$ & 1 & $4 / 7 / 95$ & 6713 & 250 & 119.7 & \\
\hline U-204 & 81 & DL & 1 & $4 / 7 / 95$ & 6884 & 40 & 21.2 & S95T000730 \\
\hline
\end{tabular}




\section{Vials in 11A1 and 11A2}

\begin{tabular}{|c|c|c|c|c|c|c|c|c|}
\hline Tank \# & Core\# & Matrix & Segment \# & Date & Jar/Vial \# & Vial Size (mLs) & Net Wt\# Found (gms) & Laboratory \# \\
\hline & & & & & & Total Weight & 140.9 & \\
\hline $\begin{array}{l}\text { U-204 } \\
\text { U-204 }\end{array}$ & $\begin{array}{l}81 \\
81\end{array}$ & $\begin{array}{l}\text { Sludge } \\
\text { Sludge }\end{array}$ & $\begin{array}{l}1 \\
1\end{array}$ & $\begin{array}{l}4 / 7 / 95 \\
9 / 8 / 97\end{array}$ & $\begin{array}{r}6883 \\
13072\end{array}$ & $\begin{array}{c}40 \\
60 \\
\text { Total Weight }\end{array}$ & $\begin{array}{c}56.6 \\
65 \\
121.6\end{array}$ & S95T000727 \\
\hline $\begin{array}{l}\text { U-204 } \\
\text { U-204 }\end{array}$ & $\begin{array}{l}82 \\
82\end{array}$ & $\begin{array}{l}\mathrm{DL} \\
\mathrm{DL}\end{array}$ & $\begin{array}{l}1 \\
1\end{array}$ & $\begin{array}{r}4 / 7 / 95 \\
4 / 12 / 95\end{array}$ & $\begin{array}{l}6783 \\
6923\end{array}$ & $\begin{array}{c}125 \\
40 \\
\text { Total Weight }\end{array}$ & $\begin{array}{l}40.1 \\
43.3 \\
83.4\end{array}$ & S95T000731 \\
\hline U-204 & 82 & FB & Fblk & $10 / 9 / 95$ & $8023^{\circ}$ & $\begin{array}{c}40 \\
\text { Total Weight }\end{array}$ & $\begin{array}{l}41 \\
41\end{array}$ & \\
\hline $\begin{array}{l}\text { U-204 } \\
\text { U-204 }\end{array}$ & $\begin{array}{l}82 \\
82\end{array}$ & $\begin{array}{l}\text { Sludge } \\
\text { Sludge }\end{array}$ & $\begin{array}{l}1 \\
1\end{array}$ & $\begin{array}{r}4 / 7 / 95 \\
10 / 31 / 95\end{array}$ & $\begin{array}{l}6784 \\
8529\end{array}$ & $\begin{array}{c}125 \\
60 \\
\text { Total Weight }\end{array}$ & $\begin{array}{l}34.5 \\
36.6 \\
71.1\end{array}$ & \$95T000729 \\
\hline
\end{tabular}


MNF-1641 REV. 0

\section{Appendix II}

\section{Inventory Database Reports and Queries Used to Support Sample and Cost Projections}




\section{Samples sorted by Vial Size}

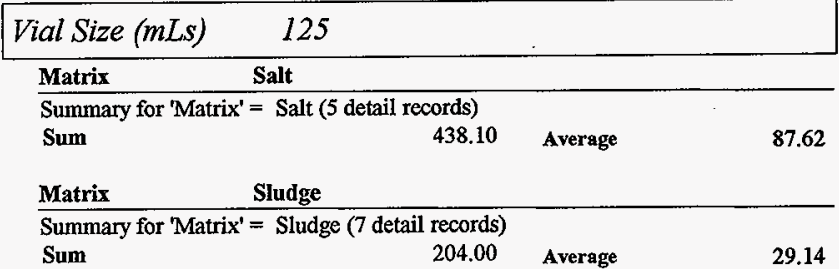

Summary for 'Vial Size (mLs)' $=125$ (12 detail records)

Sum 642.10

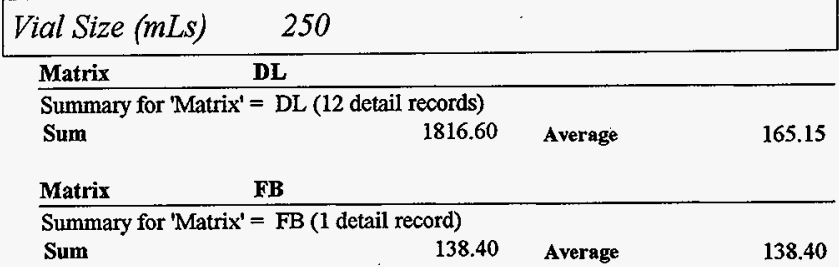

Summary for 'Vial Size (mLs)' = 250 (13 detail records)

Sum

1955.00

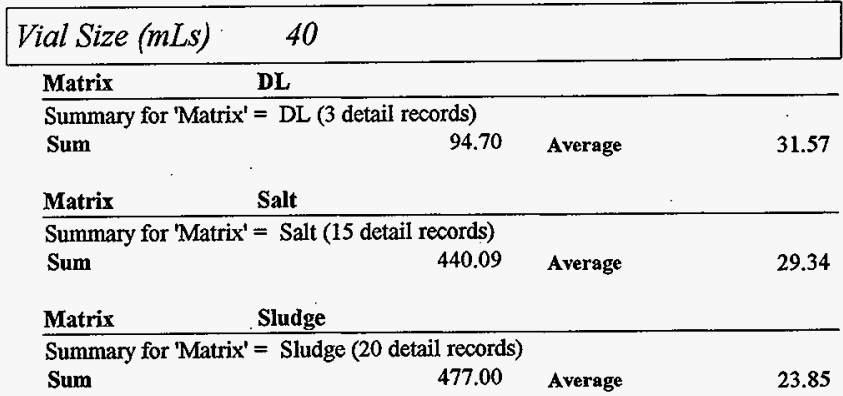




\section{HNF-1641 REV. 0}

Summary for 'Vial Size (mLs)' $=40$ (38 detail records)

Sum

1011.79

1994 Total Sample Amount (gms)

3608.89 


\section{Samples sorted by Vial Size}

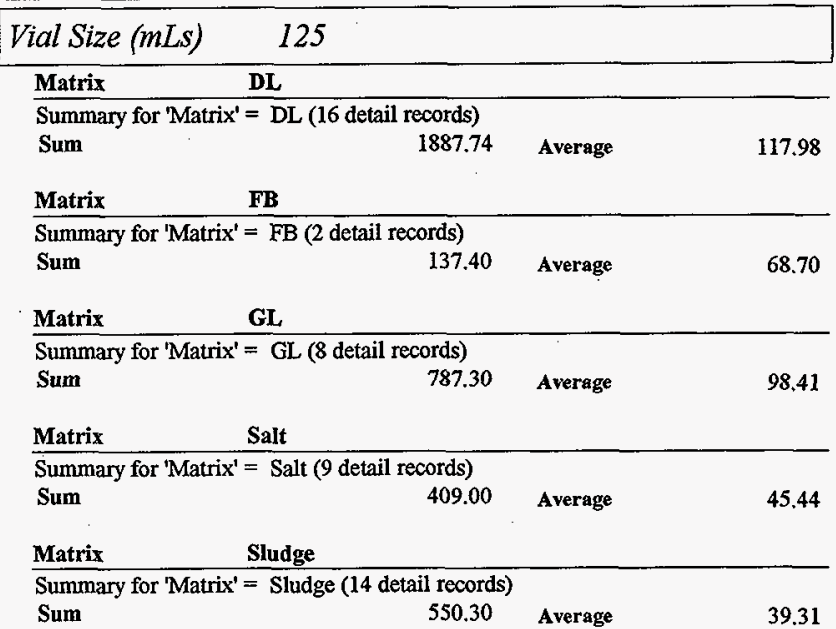

Summary for 'Vial Size (mLs)' = 125 (49 detail records)

Sum

3771.74

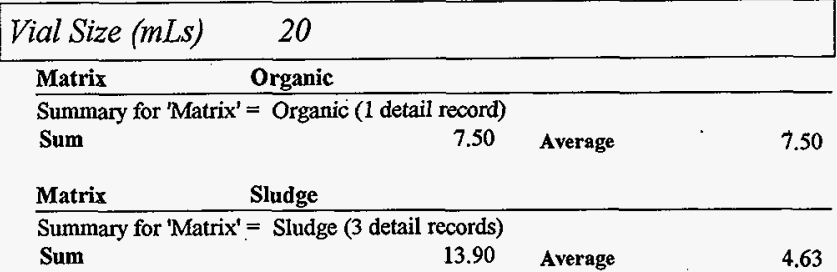

Summary for 'Vial Size (mLs)' $=20$ (4 detail records)

Sum 21.40

Vial Size (mLs) 250

Matrix DL


MNF-1641 REV. 0

Summary for 'Matrix' = DL (20 detail records)

Sum $\quad 1950.60$

Average

97.53

Matrix

FB

Summary for 'Matrix' = FB (6 detail records)

Sum

885.80

Average

147.63

Matrix

Salt

Summary for 'Matrix' $=$ Salt ( 6 detail records)

Sum

351.30

Average

58.55

Matrix

Sludge

Summary for 'Matrix' = Sludge (61 detail records)

Sum

3748.80

Average

61.46

Summary for 'Vial Size (mLs)' = 250 (93 detail records)

Sum

6936.50

\section{Vial Size (mLs) 40}

Matrix DL

Summary for 'Matrix' = DL (70 detail records)

Sum

2605.21

Average

37.22

Matrix

FB

Summary for 'Matrix' = FB (16 detail records)

Sum

549.60

Average

34.35

Matrix

GL

Summary for 'Matrix' = GL (2 detail records)

Sum

60.00

Average

30.00

Matrix

LL

Summary for 'Matrix' = LL (7 detail records)

Sum

180.90

Average

Matrix

Salt

Summary for 'Matrix' = Salt ( 82 detail records)

Sum

3583.30

Average

43.70

Matrix

Sludge

Summary for 'Matrix' = Sludge (186 detail records)

Sum

8931.87

Average

48.02

Summary for 'Vial Size (mLs)' $=40$ (363 detail records)

Sum

15910.88 
MNF-1641 REV. 0

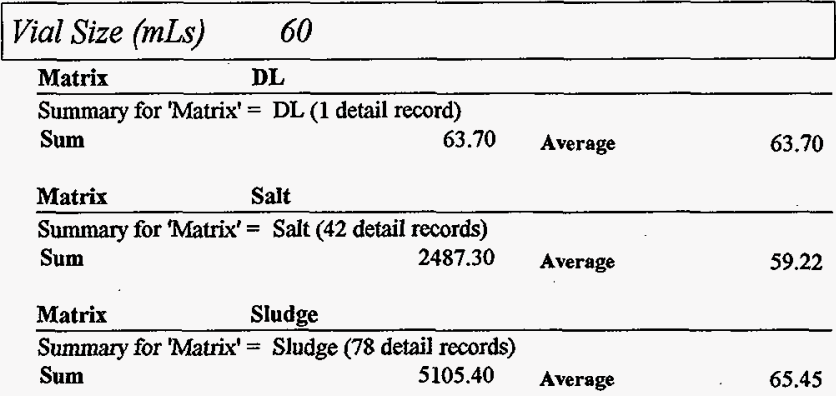

Summary for 'Vial Size (mLs)' $=60$ (121 detail records)

Sum

7656.40

\begin{tabular}{|c|c|c|c|}
\hline Vial Size (mLs) & 70 & & \\
\hline Matrix & $\mathbf{G L}$ & & \\
\hline \multicolumn{4}{|c|}{ Summary for 'Matrix' = GL (4 detail records) } \\
\hline Sum & 272.10 & Average & 68.02 \\
\hline Matrix & Salt & & \\
\hline \multicolumn{4}{|c|}{ Summary for 'Matrix' = Salt (1 detail record) } \\
\hline Sum & 40.50 & Average & 40.50 \\
\hline
\end{tabular}

Summary for 'Vial Size (mLs)' $=70$ (5 detail records)

Sum

312.60

1995 Total Sample Amount (gms)

34609.52 


\section{Samples sorted by Vial Size}

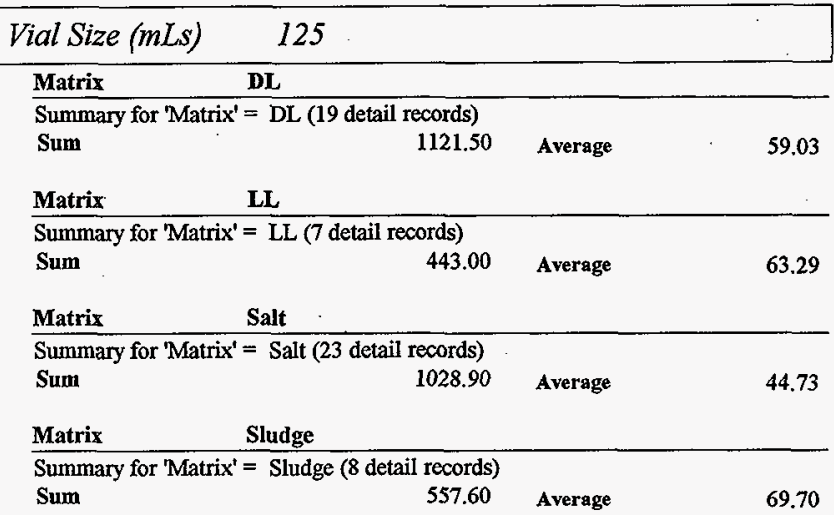

Summary for 'Vial Size (mLs)' = 125 (57 detail records)

Sum

3151.00

\begin{tabular}{|c|c|c|c|}
\hline Vial Size (mLs) & 20 & & \\
\hline Matrix & DL & & \\
\hline \multicolumn{4}{|c|}{ Summary for 'Matrix' = DL (1 detail record) } \\
\hline Sum & 3.40 & Average & 3.40 \\
\hline Matrix & GL & & \\
\hline \multicolumn{4}{|c|}{ Summary for 'Matrix' = GL (2 detail records) } \\
\hline Sum & 17.50 & Average & 8.75 \\
\hline Matrix & Salt & & \\
\hline \multicolumn{4}{|c|}{ Summary for 'Matrix' = Salt (1 detail record) } \\
\hline Sum & 7.40 & Average & 7.40 \\
\hline
\end{tabular}

Summary for 'Vial Size (mLs)' $=20$ (4 detail records)

Sum

28.30

\begin{tabular}{|c|c|}
\hline Vial Size (mLs) & 250 \\
\hline Matrix & $\overline{D L}$ \\
\hline Summary for 'Matri & $=\mathrm{DL}$ (74 detail records) \\
\hline
\end{tabular}


Matrix FB

Summary for 'Matrix' = FB (19 detail records)

Sum

2765.70

Average

145.56

Matrix

Salt

Summary for 'Matrix' $=$ Salt (90 detail records)

Sum

7789.70

Average

86.55

Matrix

Sludge

Summary for 'Matrix' $=$ Sludge (62 detail records)

Sum

5890.40

Average

95.01

Summary for 'Vial Size $(\mathrm{mLs})$ ' $=250$ (245 detail records)

Sum

31707.70

\section{Vial Size (mLs) 40}

Matrix DL

Summary for 'Matrix' = DL (112 detail records)

Sum $\quad \mathbf{5 4 3 7 . 3 0}$

Average

48.55

Matrix

FB

Summary for 'Matrix' = FB (10 detail records)

Sum

347.00

Average

34.70

Matrix

GL

Summary for 'Matrix' = GL (5 detail records)

Sum

145.00

Average

29.00

Matrix

LL

Summary for 'Matrix' = LL (11 detail records)

Sum

344.30

Average

31.30

Matrix

Salt

Summary for 'Matrix' = Salt (12 detail records)

Sum $\quad 388.30$

Average

32.36

Matrix

Sludge

Summary for 'Matrix' = Sludge (11 detail records)

Sum

514.50

Average

46.77

Summary for 'Vial Size (mLs)' $=40$ (161 detail records)

Sum

7176.40

$\begin{array}{ll}\text { Vial Size }(m L s) & 60 \\ \text { Matrix } & \text { DL }\end{array}$




\section{MNF-1641 REV. 0}

Summary for 'Matrix' = DL (41 detail records)

Sum

2597.73

Average

63.36

Matrix

FB

Summary for 'Matrix' = FB (5 detail records)

Sum

304.90

Average

60.98

Matrix

GL

Summary for 'Matrix' = GL (59 detail records)

Sum

Average

Matrix

GS

Summary for 'Matrix' = GS (2 detail records)

Sum

77.40

Average

38.70

Matrix

LL.

Summary for 'Matrix' = LI (8 detail records)

Sum

851.90

Average

106.49

Matrix

Salt

Summary for 'Matrix' = Salt (451 detail records)

Sum

30354.20

Average

67.30

Matrix

Sludge

Summary for 'Matrix' = Sludge (191 detail records)

Sum

12155.10

Average

63.64

Summary for 'Vial Size $(\mathrm{mLs}){ }^{\prime}=60$ (757 detail records)

Sum 49872.93

1996 Total Sample Amount (gms)

91936.33 


\section{Samples sorted by Vial Size}

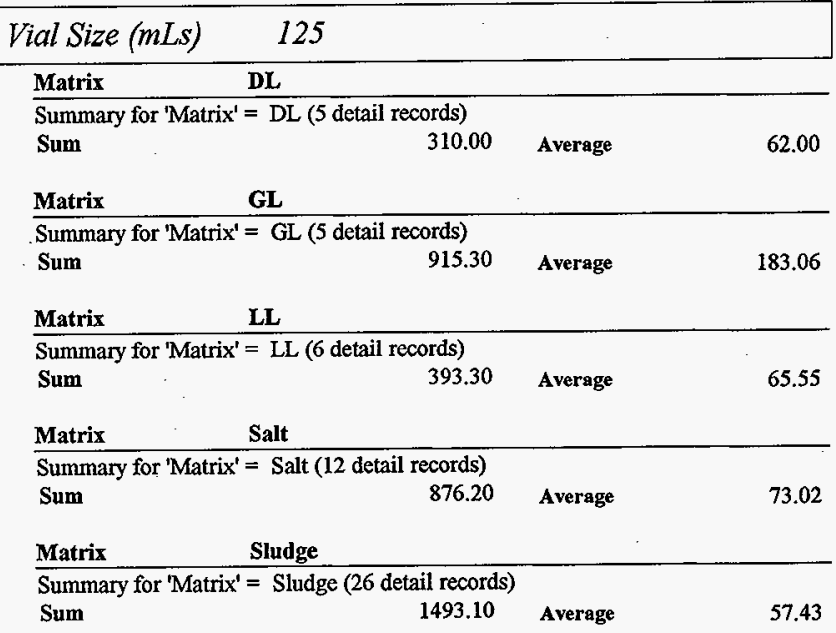

Summary for 'Vial Size (mLs)' = 125 (54 detail records)

Sum

3987.90

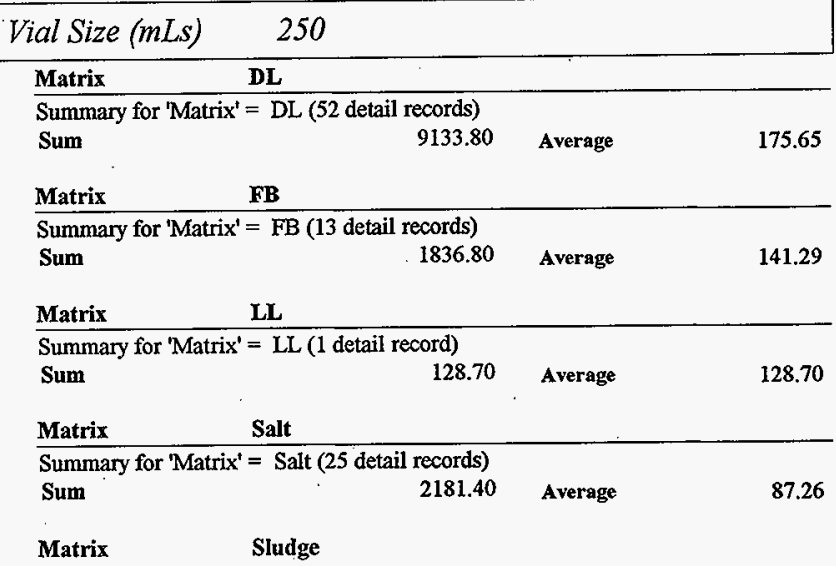


HNF-1641 REV. 0

Summary for 'Matrix' = Sludge (134 detail records)

Sum $\quad 9646.90$

Average

71.99

Summary for 'Vial Size (mLs)' $=250$ (225 detail records)

Sum

22927.60

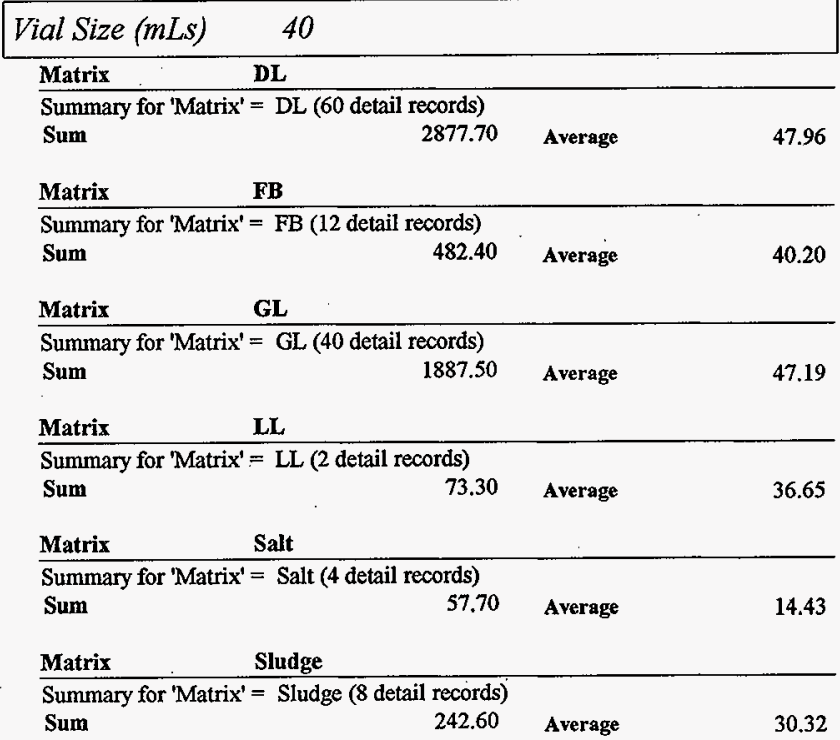

Summary for 'Vial Size (mLs)' = 40 (126 detail records)

Sum

5621.20

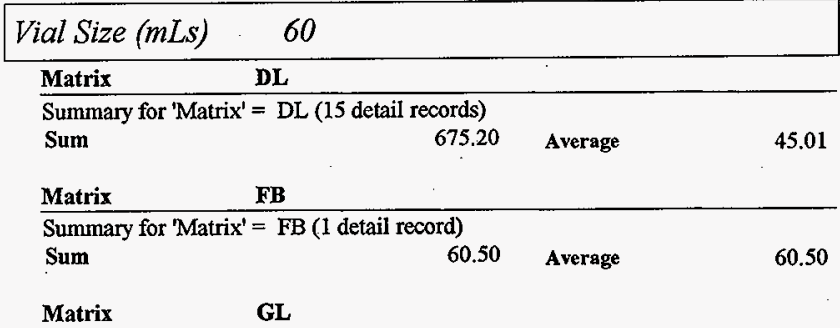


MNF-1641 REV. 0

Summary for 'Matrix' = GL ( 8 detail records)

Sum

328.60

Average

41.08

Matrix

LL

Summary for 'Matrix' = LL (4 detail records)

Sum

73.30

Average

18.32

Matrix

Salt

Summary for 'Matrix' = Salt (110 detail records)

Sum

7157.50

Average

65.07

Matrix

Sludge

Summary for 'Matrix' = Sludge (256 detail records)

Sum

17834.50

Average

69.67

Summary for 'Vial Size (mLs)' = 60 (394 detail records)

Sum

26129.60

1997 Total Sample Amount (gms)

58666.30 


\section{Samples sorted by Vial Size}

\begin{tabular}{|c|c|c|c|}
\hline Vial Size (mLs) & 125 & & \\
\hline Matrix & DL & & \\
\hline \multicolumn{4}{|c|}{ Summary for 'Matrix' = DL (1 detail record $)$} \\
\hline Sum & $74: 90$ & Average & 74.90 \\
\hline Matrix & GL & & \\
\hline \multicolumn{4}{|c|}{ Summary for 'Matrix' = GL (1 detail record) } \\
\hline Sum & 109.00 & Average & 109,00 \\
\hline
\end{tabular}

Summary for 'Vial Size (mLs)' = 125 ( 2 detail records)

Sum

183.90

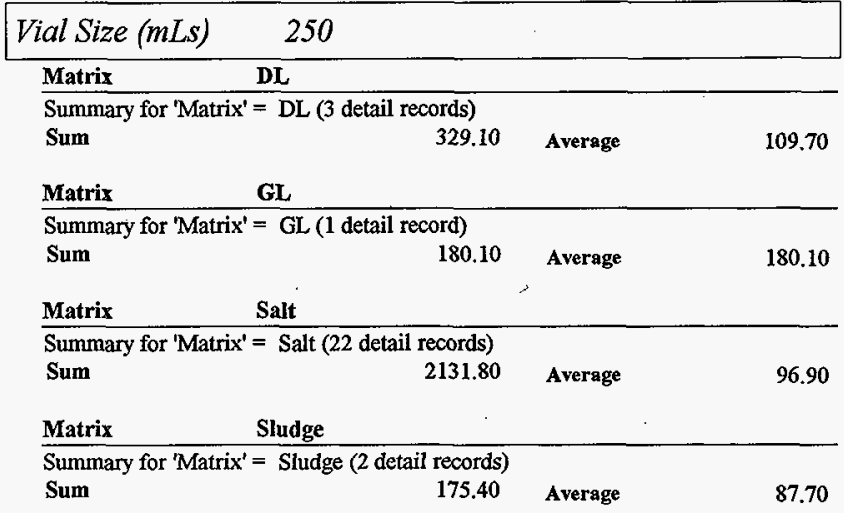

Summary for 'Vial Size (mLs)' $=250$ (28 detail records)

Sum $\quad 2816.40$

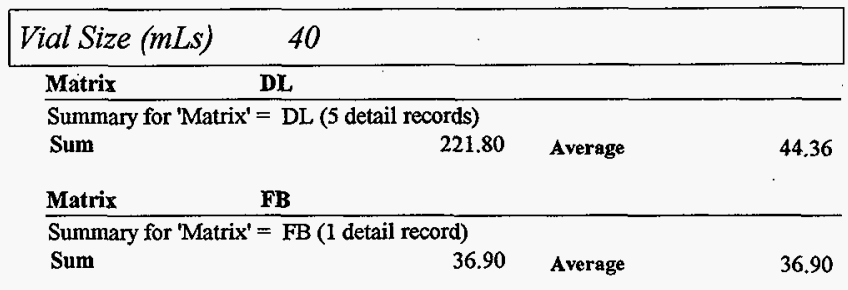




\section{HNF-1641 REV. 0}

Matrix

GL

Summary for 'Matrix' = GL (1 detail record)

Sum

40.10

Average

40.10

Matrix

LL

Summary for 'Matrix' = LL (1 detail record)

Sum

41.60

Average

41.60

Summary for 'Vial Size (mLs)' $=40$ (8 detail records)

Sum

340.40

\section{Vial Size ( $m L s) \quad 500$}

Matrix Sludge

Summary for 'Matrix' = Sludge (3 detail records)

Sum

1443.70

Average

481.23

Summary for 'Vial Size (mLs)' $=500$ (3 detail records)

Sum

1443.70

Vial Size ( $m L s) \quad 60$

Matrix DL

Summary for 'Matrix' = DL (4 detail records)

Sum

205.70

Average

51.42

Matrix

GL

Summary for 'Matrix' = GL (3 detail records)

Sum

94.10

Average

31.37

Matrix

LL

Summary for 'Matrix' = LL (2 detail records)

Sum

46.00

Average

23.00

Matrix

Salt

Summary for 'Matrix' = Salt (38 detail records)

Sum

2966.50

Average

78.07

Matrix Sludge

Summary for 'Matrix' = Sludge ( 9 detail records)

Sum 527.50

Average

58.61

Summary for 'Vial Size (mLs)' $=60$ (56 detail records)

Sum

3839.80

1998 Total Sample Amount (gms)

8624.20 


\begin{tabular}{llllrrrr} 
Tank \# & Matrix & Core\# & Segment \# & Date & Jar/Vial \# & Vial Size (mLs) & Net Wt\# Found (gus) Laboratory \# \\
\hline AW-101 & DL & R12A & & $2 / 7 / 95$ & 6427 & 40 & 4.3
\end{tabular}

\begin{tabular}{ccccccccc} 
Tank \# & Matrix & Core \# & Segment \# & Date & Jar/Vial \# & Vial Size (mLs) & Net Wt\# Found (gms) & Laboratory \# \\
\hline AW-102 & GL & Grab & & $9 / 10 / 96$ & 11275 & 20 & & 2.7
\end{tabular}

\begin{tabular}{|c|c|c|c|c|c|c|c|c|}
\hline Tank \# & Matrix & Core \# & Segment \# & Date & Jar/Vial \# & Vial Size (mLs) & Net Wt\# Found (gms) & Laboratory \# \\
\hline $\mathrm{AX}-102$ & $\mathrm{DL}$ & $\mathrm{R} 9 \mathrm{E}$ & & $3 / 1 / 95$ & 6407 & 40 & 1.92 & \\
\hline
\end{tabular}

\begin{tabular}{ccccccccc} 
Tank \# & Matrix & Core \# & Segment \# & Date & Jar/Vial \# & Vial Size (mLs) & Net Wt\# Found (gms) & Laboratory \# \\
\hline B-102 & Sludge & R1 & Auger & $10 / 19 / 94$ & 7020 & 40 & 3.2 & S95T000195
\end{tabular}

\begin{tabular}{ccccccccc} 
Tank \# & Matrix & Core \# & Segment \# & Date & Jar/Vial \# & Vial Size (mLs) & Net Wt\# Found (gms) & Laboratory \# \\
\hline B-107 & Salt & 218 & 1 & $9 / 17 / 97$ & 13169 & 60 & 2.9 & S97T002105
\end{tabular}

\begin{tabular}{ccccccccc} 
Tank \# & Matrix & Core \# & Segment\# & Date & Jar/Vial \# & Vial Size (mLs) & Net Wt\# Found (gms) & Laboratory \# \\
\hline C-103 & Sludge & 66 & 4 & $2 / 27 / 95$ & 6558 & 125 & 3.9
\end{tabular}

\begin{tabular}{lllcccccc} 
Tank \# & Matrix & Core \# & Segment \# & Date & Jar/Vial \# & Vial Size (mLs) & Net Wt Found (gms) & Laboratory \# \\
\hline C-105 & Sludge & 72 & 2 & $10 / 13 / 95$ & 8274 & 20 & S95T002854
\end{tabular}




\begin{tabular}{lcccccccc} 
Tank \# & Matrix & Core \# & Segment \# & Date & Jar/Vial \# & Vial Size (mLs) & Net Wt\# Found (gms) & Laboratory \# \\
\hline S-111 & DL & 150 & 3B & $7 / 16 / 96$ & 10562 & 20 & 3.4 & S96T004187
\end{tabular}

\begin{tabular}{ccccccccc} 
Tank \# & Matrix & Core \# & Segment \# & Date & Jar/Vial \# & Vial Size (mLs) & Net Wt\# Found (gms) & Laboratory \# \\
\hline SX-104 & GL & Grab & $97-1$ & $2 / 11 / 98$ & 14010 & 60 & 4.8 & S98T000428
\end{tabular}

\begin{tabular}{ccccccccc} 
Tank \# & Matrix & Core \# & Segment \# & Date & Jar/Nial \# & Vial Size (mLs) & Net W\# Found (gms) & Laboratory \# \\
\hline SX-108 & Sludge & R16 & Aug-43 & $10 / 2 / 95$ & 8115 & 20 & 3.5 & S95T002577
\end{tabular}

\begin{tabular}{lllcccccc} 
Tank \# & Matrix & Core\# & Segment \# & Date & Jar/Vial \# & Vial Size (mLs) & Net Wt\# Found (gms) & Laboratory \# \\
\hline SY-103 & Salt & 62 & 6 & $11 / 11 / 94$ & 7035 & 40 & 3.5 &. \\
SY-103 & Sludge & 62 & 15 & $9 / 26 / 94$ & 6169 & 40 & 4.6 & S94T000102
\end{tabular}

\begin{tabular}{lcccccccc} 
Tank \# & Matrix & Core \# & Segment \# & Date & Jar/Vial \# & Vial Size (mLs) & Net Wt\# Found (gms) & Laboratory \# \\
\hline T-203 & DL & 190 & IR & $4 / 28 / 97$ & 12623 & 60 & 1.2 & S97T000730
\end{tabular}

\begin{tabular}{lllcccccc} 
Tank \# & Matrix & Core \# & Segment \# & Date & Jar/Vial \# & Vial Size (mLs) & Net Wt\# Found (gms) & Laboratory \# \\
\hline U-105 & LL & 133 & 1 & $3 / 1 / 96$ & 9339 & 60 & 4.1 & S96T001235 \\
U-105 & Salt & 131 & 2 & $3 / 14 / 96$ & 9541 & 60 & 4.6 & S96T000936
\end{tabular}




\begin{tabular}{lllcrrrrr} 
Tank \# & Matrix & Core \# & Segment \# & Date & Jar/Vial \# & Vial Size (mLs) & Net Wt\# Found (gms) & Laboratory \# \\
\hline U-107 & Salt & 134 & 5 & $3 / 12 / 96$ & 9548 & 60 & 2.1 & S96T001052 \\
U-107 & Salt & 134 & $6 A$ & $3 / 1 / 96$ & 9338 & 60 & 1.9 & S96T001069
\end{tabular}

\begin{tabular}{lllccrrrr} 
Tank \# & Matrix & Core \# & Segment \# & Date & Jar/Vial \# & Vial Size (mLs) & Net Wt\# Found (gms) & Laboratory \# \\
\hline U-108 & Salt & 146 & 5 & $5 / 7 / 96$ & 10020 & 250 & S96T002954
\end{tabular}




\section{5 and $250 \mathrm{~mL}$ vials which can be repacked into $60 \mathrm{~mL}$ vials}

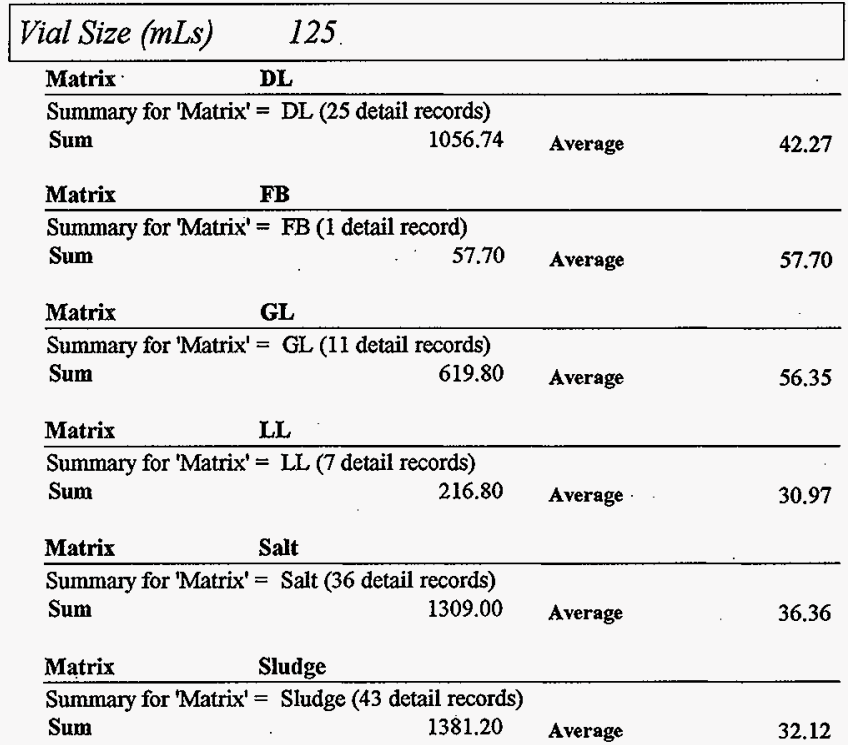

Summary for 'Vial Size (mLs)' = 125 (123 detail records)

Sum

4641.24

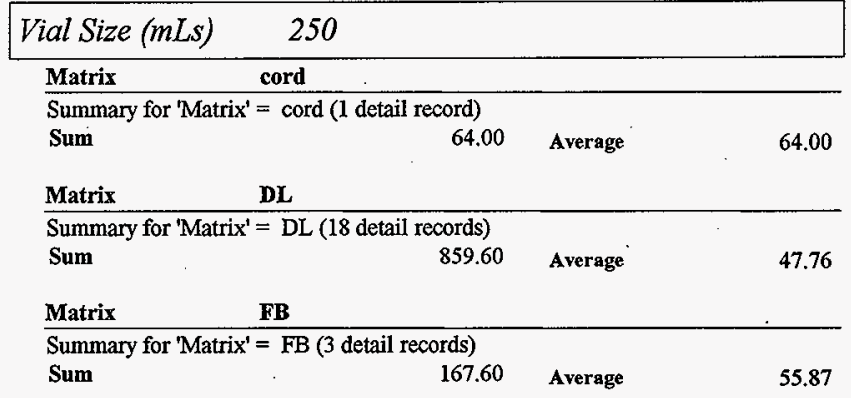


HNF-1641 REV. 0

Matrix

Salt

Summary for 'Matrix' = Salt (58 detail records)

Sum

3031.20

Average

52.26

Matrix

Sludge

Summary for 'Matrix' = Sludge (166 detail records)

Sum

8118.00

Average

48.90

Summary for 'Vial Size $(\mathrm{mLs})^{\prime}=250$ (246 detail records)

Sum

12240.40

Total Sample Amount (gms)

16881.64 


\section{$250 \mathrm{~mL}$ vials which can be repacked into $125 \mathrm{~mL}$ vials}

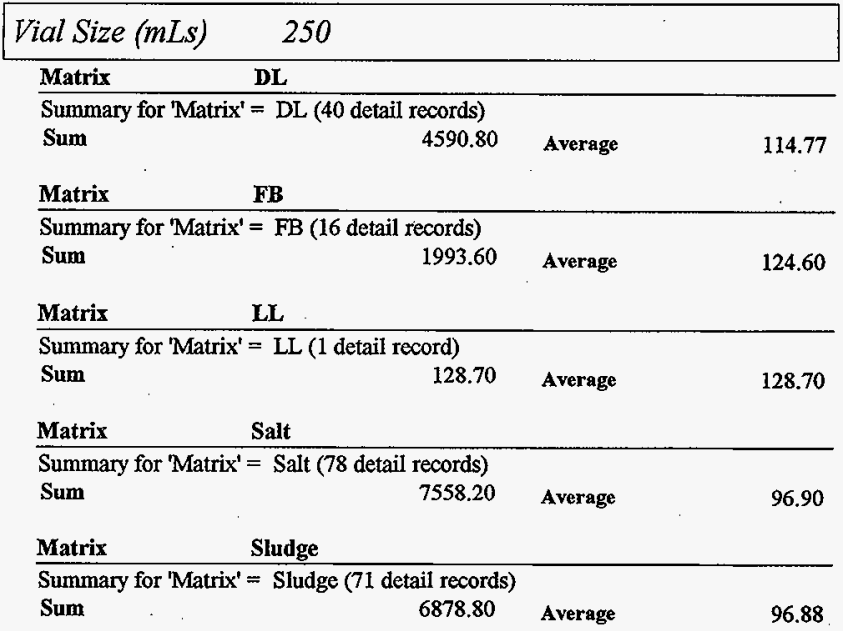

Summary for 'Vial Size (mLs)' $=250$ (206 detail records)

Sum

21150.10

Total Sample Amount (gms)

21150.10 
DISTRIBUTION SHEET

\begin{tabular}{|c|c|c|c|c|c|}
\hline \multirow{2}{*}{$\begin{array}{l}\text { To } \\
\text { Distribution }\end{array}$} & \multirow{2}{*}{\multicolumn{3}{|c|}{$\begin{array}{l}\text { From } \\
\text { Production Planning \& Control }\end{array}$}} & \multicolumn{2}{|c|}{ Page 1 of 1} \\
\hline & & & & \multicolumn{2}{|c|}{ Date Apri] 21, 1998} \\
\hline \multicolumn{4}{|l|}{ Project Title/Work Order } & \multicolumn{2}{|c|}{ EDT No. EDT-623151 } \\
\hline \multicolumn{4}{|c|}{$\begin{array}{l}\text { HNF-1641, Rev. 0. "Development of an Inventory/Archive Program } \\
\text { for the Retention, Management, and Disposition of Tank } \\
\text { Characterization Samples at the 222-S Laboratory" }\end{array}$} & \multicolumn{2}{|l|}{ ECN No. $\quad N / A$} \\
\hline Name & MSIN & $\begin{array}{l}\text { Text } \\
\text { With All } \\
\text { Attach. }\end{array}$ & Text Only & $\begin{array}{l}\text { Attach./ } \\
\text { Appendix } \\
\text { Only }\end{array}$ & $\begin{array}{l}\text { EDT/ECN } \\
\text { Only }\end{array}$ \\
\hline \multicolumn{6}{|c|}{$\begin{array}{l}\text { Fluor Daniel Hanford, Inc. } \\
\text { A. G. Mishko }\end{array}$} \\
\hline $\begin{array}{l}\text { Lockheed Martin Hanford } \\
\text { M. R. Adams } \\
\text { J. G. Burton } \\
\text { K. M. Hal1 } \\
\text { J. G. Kristofzski } \\
\text { W. E. Ross }\end{array}$ & $\begin{array}{l}\text { R2-12 } \\
\text { S7-21 } \\
\text { R2-12 } \\
\text { R2-12 } \\
\text { S7-84 }\end{array}$ & $\begin{array}{l}X \\
X \\
X \\
X \\
X\end{array}$ & & & \\
\hline \multicolumn{5}{|l|}{ Numatec Hanford Corp. } & \\
\hline $\begin{array}{l}\text { Waste Management Federa } \\
\text { R. Akita } \\
\text { S. L. Brey } \\
\text { J. E. Hyatt } \\
\text { J. B. Buckley } \\
\text { D. S. Mantooth } \\
\text { M. F. Marcus } \\
\text { G. B. Svancara } \\
\text { K. S. Tollefson } \\
\text { S. J. Turner } \\
\text { J. L. Westcott } \\
\text { J. A. Winterhalder }\end{array}$ & $\begin{array}{c}\text { of Hanford, I } \\
\text { T6-20 } \\
\text { T6-04 } \\
\text { T6-14 } \\
\text { T3-04 } \\
\text { T6-03 } \\
\text { S3-28 } \\
\text { T6-03 } \\
\text { T6-12 } \\
\text { T6-12 } \\
\text { T3-04 } \\
\text { H6-21 } \\
\end{array}$ & $\begin{array}{l}X \\
X \\
X \\
X \\
X \\
X \\
X \\
X \\
X \\
X \\
X\end{array}$ & & & \\
\hline
\end{tabular}

